\title{
STUDIES ON THE DEVELOPMENT \\ AND OSTEOLOGY OF SOME \\ NEW ZEALAND INSHORE FISHES
}

By J. G. RUCK

\section{Aum}

Submitted for the degris of

Doctor of Philosophy (Zoology)

at Victoria University of Wellington, New Zealand.

May, 1976. 
- FRONTISPIECE -

Left lateral aspect of skull of Helcogramma sp.






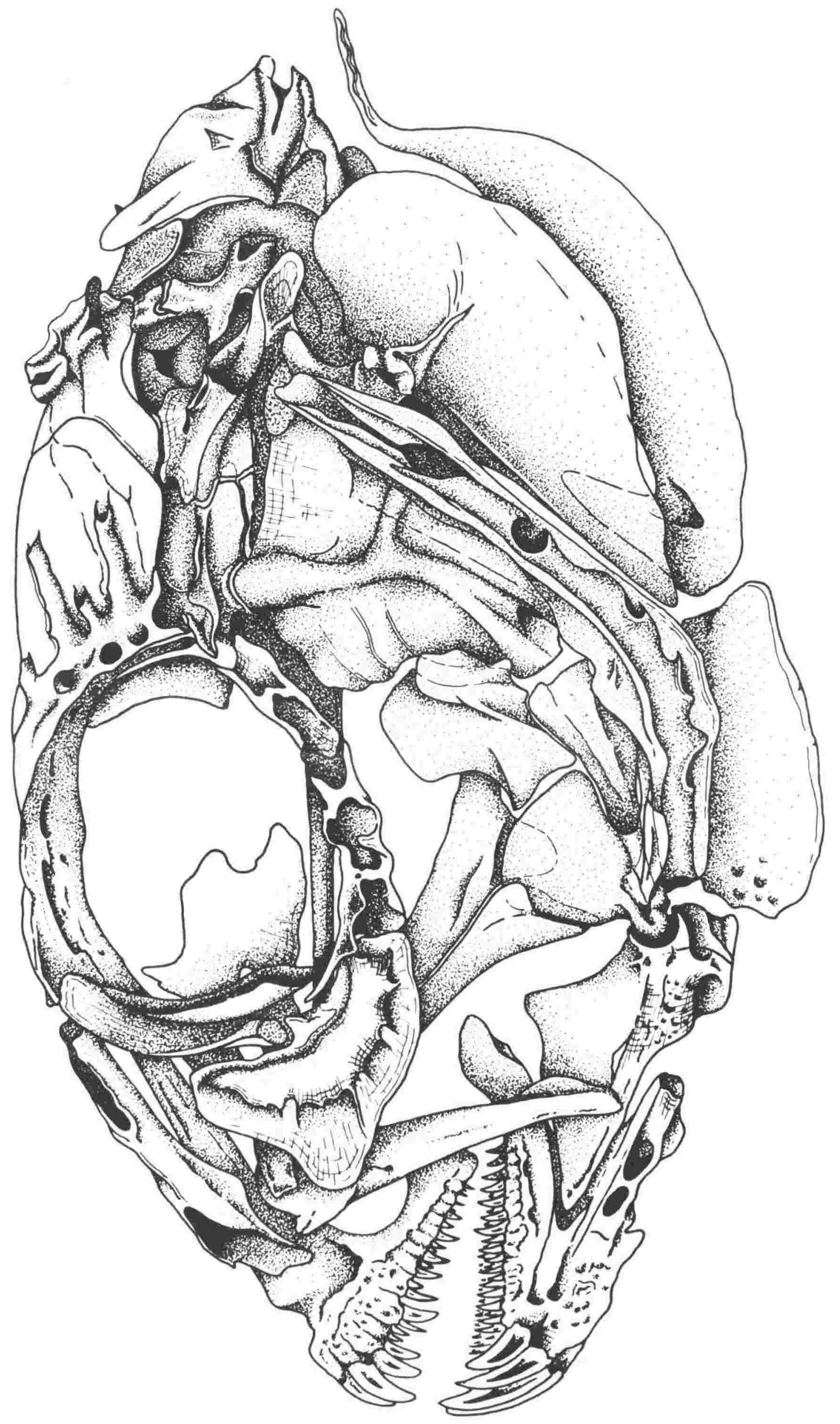


CONTENTS

Abstract

Page No.

Acknowledgements

Introduction

Clarification of adult nomenclature

Osteological section

Introduction

Adult skeleton of Forsterygion varium

Discussion - adult skeleton

Larval skeleton of Forsterygion varium

Discussion - larval skeleton

Osteological figures

Embryological section

Trachelochismus melobesia

Forsterygion capito (Tripterygion)

Forsterygion nigripenne (Tripterygion robustum)

Diplocrepis puniceus

Trachelochismus pinnulatus

Forsterygion varium

Gilloblennius decemdigitatus

Gilloblennius tripennis

Discussion - embryological development

Key to tripterygiid and gobiesocid eggs

Prolarval, larval and prejuvenile section

Introduction

Tripterygiidae

Clinidae

Gobiesocidae

Discussion - larval development

Key to tripterygiid, clinid and gobiesocid larvae and prejuveniles

Prolarval, larval and prejuvenile plates 


\section{Abstract}

This study deals principally with the problems involved in the identification of the early stages of tripterygiid, clinid and gobiesocid fish in the Cook strait region, New Zealand.

The nomenclature of 7 tripterygiid and 4 clinid species is reviewed to assist in the identification of the developmental stages. Those species reviewed are preceded by an asterisk in the list included later in this abstract.

The adult osteology of Forsterygion varium (Forster in Bloch and Schneider, 1801) is described and illustrated in detail, together with the osteology of the larvae and prejuveniles at different stages of development. The adult osteology is compared with that of other blennioid fishes. In $F$. varium the skeletal elements begin to form over a wide range of larval sizes and full adult osteological characters are acquired at a variable time after prejuvenile development is reached. Functionally related elements tend to attain their adult form at about the same size. There is no correlation between the size at initial ossification and the endochondral or dermal origin of a bone. Elements of the vertebral column and median fins develop sequentially and therefore provide (in tripterygiids) an index of development which is useful in comparing the larval stages of different species.

The embryological stages of 5 tripterygiid and 3 gobiesocid fish are described and illustrated in detail. Species studied are: Forsterygion capito (Tripterygion capito) (Jenyns, 1842), Forsterygion nigripenne (Tripterygion robustum) (Valenciennes in Cuvier and Valenciennes, 1836), Forsterygion varium (Forster in Bloch and Schneider, 1801), Gilloblennius decemdigitatus (Clarke, 1879), G. tripennis (Forster in Bloch and Schneider, 1801), Trachelochismus melobesia Phillipps, 1927, T. pinnulatus (Forster in Bloch and Schneider, 1801), Diplocrepis puniceus (Richardson, 1846). The eggs of all species are laid on submerged objects in clusters of 20-3000 eggs in shallow coastal water. Eggs are attended by an adult fish until hatching occurs. Prolarvae are well developed with fully pigmented eyes, functional jaws and reduced yolk-sacs, and are therefore useful in linking later larval stages with adults. 
An assessment of the general problems encountered in the identification of larvae and prejuveniles is presented with attention given to the fish in this study. The larval and prejuvenile stages of 10 tripterygiid, 4 clinid and 9 gobiesocid species are described and illustrated in detail. Those described are - Tripterygiidae: *Forsterygion varium (Forster in Bloch and Schneider, 1801), *F. nigripenne (Valenciennes in Cuvier and Valenciennes, 1836), *F. capito (Jenyns, 1842), *Tripterygion segmentatum McCulloch and Phillipps, 1923, *Gilloblennius decemdigitatus (Clarke, 1879), *G. tripennis (Forster in Bloch and Schneider, 1801), *Helcogramma medium (in part Güther, 1861), two Helcogramma species, new tripterygiid species (genus not certain); Clinidae: *Notoclinus compressus (Hutton, 1872), *N. fenestratus (Forster in Bloch and Schneider, 1801), *Ericentrus rubrus (Hutton, 1872), *Cologrammus flavescens (Hutton, 1872); Gobiesocidae: Trachelochismus melobesia Phillipps, 1927, T. pinnulatus (Forster in Bloch and Schneider, 1801), Diplocrepis puniceus (Richardson, 1846), Diplocrepis puniceus (South Island form), Gastroscyphus hectoris (Günther, 1876), Gastroscyphus species, Gastrocyathus gracilis Briggs, 1955, Dellichthys morelandi Briggs, 1955, Haplocylix littoreus (Eorster in Bloch and Schneider, 1801). A key to the larvae and prejuveniles of the above species is included.

Closely related tripterygiid species with very similar larval stages were identified mainly, by considering myomere counts and by consistent differences in size (standard length) at given stages of development. In contrast clinid and gobiesocid larvae from unrelated adults were readily distinguished by a wide range of characteristics.

Larvae and prejuveniles were collected using standard equipment such as nylon mesh plankton nets and light-traps. A light-trap designed specifically for collecting larvae is described in detail in the appendix.

An annotated bibliography of New Zealand teleost eggs and larvae is presented in the appendix with reference to 70 marine and freshwater species. 


\section{ACKNOWLEDGEMENTS}

I would like to thank sincerely the following people for their assistance: Dr P.H.J. Castle, Victoria University of Wellington to whom I am grateful for his general supervision, ample encouragement and constructive criticism; Prof. J.A.F. Garrick, Victoria University of Wellington for his overall interest, constructive criticism and for allowing me the use of equipment within the zoology Department; Mr J. Moreland, National Museum for his considerable information about the systematics of tripterygiid and clinid fishes and for allowing me to use some of the specimens he had collected; Mr G. Grainger, Technician at the Marine Laboratory, Island Bay who helped design and construct special collecting and holding apparatus; Mr W. McQueen, Master of the University Research Vessel Tirohia for his help on numerous collecting trips; Mr D. Robertson, Fisheries Research Division who gave me samples of tripterygiid and gobiesocid larvae collected from South Island waters; Mr G. Anderson, University of Auckland who gave valuable information about the reproductive behaviour and systematics of tripterygiid fish; Mrs singleton who typed this thesis and numerous other friends who helped throughout this study.

Finally I wish to thank my wife Annette, who has had the misfortune of tolerating the tensions and frustrations inherent within this sort of project. 


\section{INTRODUCTION}

The statement by Mansueti and Hardy (1967) that "Knowledge of early developmental stages of fishes is obviously fundamental to proper understanding of many aspects of fishery biology and ichthyology", is one of a number which in recent years has acknowledged the value of studies on the early life histories of fishes. It is now well recognised that accurate identification of the eggs and larvae of fishes is necessary for defining breeding areas, understanding the distribution and biology of larvae, protecting young stages, carrying out experimental research and assisting in adult classification. All of these facets should, ideally, be investigated since they contribute to our understanding of the overall biology of fishes, and especially of commercially important or endangered species.

Descriptions of egg and larval series, exemplifying recent research, include accounts by Berry (1959), Norden (1961), Orton (1963), Peckham (1963), Mansueti A. J., (1964), Mansueti R. J. (1964), Ryland (1966), Mansueti and Hardy (1967) - (the last named being an atlas of eggs and larvae of $45 \mathrm{fish}$ species and containing numerous references to other works) - Leonard (1971), Moser (1972), Moser and Ahlstrom (1972), Aprieto (1974), Miller and Sumida (1974) and Berrien (1975). Problems associated with collecting early stages for descriptive and ecological work have received attention from Colton et al., (1961), Barkley (1972), Murphy and Clutter (1972) and Leonarz (1972) who deal with aspects such as the effectiveness of tow nets, the value of using fine mesh purse seine nets and the ability of different meshes to retain larvae.

Compared with eggs and larvae collected from the plankton, laboratory reared material can more positively and easily be identified, i.e. matched with parent species and with other specimens in the one series. In view of this it is not suxprising that much effort has been given to the development of rearing techniques such as those described in Clemens (1956), Riley and Thacker (1963), Shelbourne (1963), Shelbourne et al., (1963) - (these three papers mark the somewhat revolutionary increase in the use of Artemia salina (brine shrimp) as a larval food source) - Fahy (1964), Blaxter (1968a), Delmonte et al., (1968), May (1970), Adron et al., (1974) and Miller and Sumida (1974). May (1970) reviews the nany and varied methods of feeding larvae. 
Improved rearing techniques have stemmed from studies on larval behaviour, and in particular on feeding (Miller et al., 1963; Blaxter, 1968b; Detwyler and Houde, 1970; Nordeng and Bratland, 1971; Hunter, 1972; Kjelson et al., 1975). Experimental studies on the effects of temperature and salinity on eggs and larvae (Bishai, 1961; May, 1975) have also improved rearing techniques and contributed to our general knowledge of eggs and larvae.

Information gained from descriptive studies has been used in systematics by Orton (1953b, 1963), Castle (1969), and Moser and Ahlstrom (1972, 1974) and in population biology by Tibbo et al., (1958), Raitt (1964), Smith (1972), and Kendall and Reintjes (1975).

The most widely used method for identification of ontogenetic stages is to build up egg and larval series with specimens collected from the plankton. The various stages are linked either by relatively stable characteristics or by distinctive features whose gradual change may be traced without difficulty e.g. photophores and pigmentation, myomere counts, fin rays and spines, gillrakers and teeth etc. An account of the larval characteristics used in the identification of species in general and those herein studied is given in the larval section of this thesis. The linking of different ontogenetic stages is often difficult because of the many changes in form and structure which occur during development from egg to adult. Frequently the young stages cannot readily be identified because they have not yet acquired characteristics present in the older stages. These distinguishing characteristics include such items as pigmentation patterns, lateral line scales, rays, spines, vertebrae, overall adult shape and size, or specialized structures like suckers, scutes and tentacles. Difficulty in identification is accentuated where closely related and often morphologically similar species are being compared, as for example in the cyprinids (Balinsky, 1948), myctophids (Moser and Ahlstrom, 1974), carangids (Berry, 1959; Aprieto, 1974) and centrarchids (Applegate, 1966).

Although world literature dealing with the identification of the early stages of fishes is extensive, only a few authors such as Berry (1959), Runyan (1961), Mansueti and Hardy (1967), Aprieto (1974) and Miller and Sumida (1974) have dealt with more than one or two aspects of development. Most authors concentrate on either the eggs or the larvae or on limited aspects of both. 
Aims of the present study include highlighting the main problems encountered in building up egg and larval series of fishes, and attempting to provide practical solutions to these problems. None of the problems here encountered is particularly new to developmental research. Because the study deals with closely related and morphologically similar species, existing solutions to problems of identification (see descriptive references above) have had to be refined in some cases to suit the situation. It is appreciated that no single study could hope to cover every obstacle likely to arise, as the latter are as many and varied as the fish species themselves. Nevertheless it is believed that the techniques illustrated here (some of them new, others as refinements and extersions of existing methods) will have fairly general application in developmental work. A further aim was to provide this study with as broad a foundation as possible, and for this reason attention is given to:- the methods of collection, preservation, clearing and staining of delicate specimens; techniques for keeping eggs, larvae and adults alive for observation; the definitions of developmental stages so that different species may be compared; the clarity and accuracy of anatomical terminology; and the adult classification, which, if inaccurate, compounds problems of egg and larval identification.

To achieve the above aims, the fishes of three inshore families, Tripterygiidae, Clinidae and Gobiesocidae, were chosen for study for the following reasons. Firstly, the three families should give adequate scope for intra and inter familial comparison of developmental stages. It was reasoned that if new or refined techniques could be applied successfully to the members of these three families, then they should apply equally well to fishes in general.

Secondly, a desirable feature of any study is to have ready availability of the study material. Experience had shown that adult tripterygiids, clinids and gobiesocids were easily captured and could be kept alive in aquaria for considerable lengths of time. Stocks of adults for "natural" and artificial spawning were, therefore, always on hand. The eggs of many inshore fishes, and in particular the tripterygiids, clinids and gobiesocids, are laid on or beneath objects within the tidal range (Graham, 1939, 1953; Coakley, 1964; Darby, 1966; Gibson, 1969). 
It was known (above references and personal experience) that these eggs could be hatched - with limited mortality - in aquaria. It was also appreciated that parental care of egg masses was common (Graham, 1939, 1953; Gibson, 1969) and that the presence of adults near eggs would help in their identification (Jillet, 1968). The adult tripterygiids, clinids and gobiesocids are abundant in shallow coastal waters of New Zealand. It was assumed that the larvae would also be abundant and comparatively close inshore. Trial and subsequent plankton tows proved this assumption to be correct.

Thirdly, it was felt that information on tidal fishes and their dependence on tidal zones for reproduction etc., would greatly improve our understanding of the fishes in such an accessible environment. The tidal zone is a relatively harsh environment where extremes of physical conditions are experienced, including turbidity and pounding water. This narrow zone supports a diverse population of fishes, each with interesting adaptations which ensure their survival. Perhaps some of the most important adaptations involve reproduction where a variety of methods are used ranging from the release of pelagic eggs to the laying of eggs beneath objects with subsequent protection by parental care, to live bearing.

When this study was begun it was believed that the classification of the New Zealand species within the three families to be studied should present few problems. However, it was soon discovered that the nomenclature of the tripterygiids in particular, and to a lesser extent that of the clinids, was confused. Embryological and larval studies would, therefore, be restricted until the identification and nomenclature had been clarified, as separation of the species of larvae etc., would be difficult enough without the added confusion in adult identification and classification. For this reason the first section of this thesis is a partial review of the New Zealand tripterygiid and clinid species.

Following the review is a section devoted to the larval and adult osteology of Forsterygion varium (Forster in Bloch and Schneider, 1801). The main purpose of the osteological study was to provide information on the sequence and timing of the development of meristic structures such as spines, rays and vertebrae, for use in identifying the larval stages of some New Zealand tripterygiids, clinids and gobiesocids. Further explanation for including larval and adult osteology in this study is 
given in the introduction to the section on osteology.

Following the osteology there is a section on embryology which is presented as a series of papers. A study of embxyology forms a logical forerunner to larval research insofar as a knowledge of egg stages is of value in identifying larval stages (Delsman, 1923; Mansueti, R. J. 1964; Mansueti, A. J., 1964; Baker, 1972, 1973). This is particularly true in the three families chosen, where prolarval development at hatching is very advanced and prolarvae are quite easily matched with later stages. The embryological work was carried out over several years, and when the study of each egg series was completed it was published. The order in which each species was dealt with depended mainly on the availability of study material. Some of the publications dealing with common species predate work done on the taxonomic review of the New Zealand tripterygiids and clinids. These facts account for two anomalies in the embryological section, these being the separation of the accounts of congeneric species (i.e. Trachelochismus melobesia Phillipps, 1927, and T. pinnulatus (Forstex in Bloch and Schneider, 1801) appear in separate papers), and the placement of capito and robustum in the genus Tripterygion instead of Forsterygion as in the review.

The embryological papers exhibit a progression of skill, from early papers where trial and error techniques were used to later papers which exhibit a more complete understanding of embryology. The series is an expression of evolving technique and understanding.

A section on larval studies which follows the embryological work is, perhaps, the most important section; it represents a synthesis of the preceding research because it relies heavily on principles and conclusions arrived at in earlier sections. Here the problems of larval classification are outlined and solutions to these are proposed. These solutions are used extensively in the identification of many of the tripterygiid, clinid and gobiesocid larvae studied.

An annotated bibliography of New zealand teleost eggs and larvae is included in the appendix. The present study was begun at a time when information on New Zealand fish eggs and larvae was scattered, and the aim of the bibliography was to draw together this scattered information on developmental stages. Since the time the bibliography was completed considerable information on the early stages of New Zealand marine fishes 
has been compiled by Dr D. A. Robertson, Fisheries Research Division, Wellington (Robertson, 1973).

An article describing a light-trap for collecting marine fish larvae and small invertebrates is also included in the appendix. This light-trap was designed to meet the requirements of this study.

All references cited in the text are listed at the end of the thesis, with the exception of those already given at the end of each published paper on embryology and the light-trap, and those contained within the list of references in the bibliography.

Members of the Tripterygiidae, Clinidae (suborder Blennioidei) and Gobiesocidae (order Gobiesociformes) are relatively common fishes of tidal zones and shallow reef systems of the New Zealand coast. The tripterygiids are well represented by over 20 species, outnumbering the related clinids which have 6 species. There are 8 known endemic gobiesocids. The 3 families are distinguished by the following characteristics.

Tripterygiids have a 3 part dorsal fin, the anterior two of spines and the posterior one of soft rays. They are all poor swimmers and relatively sedentary. An undescribed "oblique-swimming blenny" is unusual in that it spends considerable time in small schools just above the bottom. The pelvic fins are jugular and reduced to two main rays which when extended act as struts to lift the front of the fish from the bottom. Coupled with this the eyes are high on the head, giving the fish good all round vision. Swimming is restricted to short hops. The fish is launched from the pelvics by beating the tail and large pectorals, just sufficient to maintain an oblique attitude above the bottom. When alarmed however the pelvics and pectorals are drawn close to the body and the tail beats rapidly sending the animal forward in normal fish-like motion. New zealand tripterygiids occupy a wide range of habitats throughout the tidal zone, being noted for their tolerance of physical extremes, in particular Helcogramma medium (Günther, 1861) (Darby, 1966). They eat a wide variety of food items: larvae of the marine mosquito opifex fusca Hutton, amphipods, copepods, isopods, gastropods, barnacle cirri and polychaetes (Darby, 1966, Ruck, 1968). Scavenging is common, although they prefer live food. Sexual dimorphism (mainly in colour) is usual particularly during the breeding season, which is broadly from midwinter to late summer. The eggs are laid on or under some submerged object and are usually attended by the 
male adult until hatching. A wide variety of inter-specific colour patterns is exhibited by the tripterygiids, the vividness of which tends to increase northwards. Intraspecific variation in colours is also considerable, where the intensity and type of pigmentation appears influenced by the predominant colour in the habitat.

There are two types of clinids in New Zealand. Some are similar to the tripterygiids in that they are oviporous and have 3 parts to the dorsal fin. Others have continuous spinous dorsal fins and are ovoviviparous. They swim with a sinusoidal motion and they have jugular pelvics which are capable of independent movement allowing the fish to "walk" along the bottom. The eyes are prominent and also capable of independent movement. Minute embedded scales cover the body and there is an intramittent organ in the male.

All clinids are secretive algal dwellers and have pigmentation that matches the greens, yellows and browns of seaweed fronds. Notoclinus compressus (Hutton, 1872) has a peculiar habit of swimming upside down when released from algal cover. The clinids are essentially seaweed browsers consuming small copepods, amphipods and isopods.

The gobiesocids, in contrast to the tripterygiids and clinids, have their pelvic fins modified as suckers. They are secretive fishes preferring to attach themselves to algal fronds or the underside of rocks etc. The weed-dwelling gobiesocids are more slender and smaller than the more robust rock-dwellers. Gobiesocids feed on small invertebrates (Coakley, 1964). The breeding season extends from midwinter to midsummer.

More general information about the different species of the 3 families is found in the following: general characteristics and spawning (Graham, 1937, 1939, 1953); ovoviviparity (Moreland and Dell, 1950); parasites (Laird, 1951, 1953; Hewitt, 1964; Hewitt and Hine, 1972); taxonomy of the gobiesocids (Briggs, 1955); clingfish ecology (Coakley, 1964); metabolism (Morris, 1965); general biology (Heath and Moreland, 1967); coexistence of two tripterygiid species (Ruck, 1968); general biology of the tripterygiids (Doak, 1972). 


\section{Clarification of adult nomenclature}

This section is intended to clarify the nomenclatuxe of the $23 \mathrm{fish}$ species studied in this thesis. Ten of these species belong in the family Tripterygiidae, four in the Clinidae and nine in the Gobiesocidae. All these families are teleostean fishes belonging to the cohort Euteleostei (Rosen, 1973).

The tripterygiids and clinids are nembers of the superorder Acanthopterygii (Greenwood et.al., 1966) a group of advanced euteleosteans comprising "...about $35 \%$ of all living fishes and some 7000 species..." (Cohen, 1970; Rosen, 1973). In terms of interrelationships between families etc., this immense group of fishes is still poorly understood. of the 12 orders within the Acanthopterygii the tripterygiids and clinids belong to the largest, $\mathrm{n}$ amely the Perciformes. They are blennioid fishes (suborder Blennioidei) of the superfamily Blennioidae with the controversial characteristics (Springer and Freihofer, in press) outlined by Gosline (1968). The various relationships of families within the Blennioidae will be discussed in greater detail in the later section on osteology.

The gobiesocids in contrast belong to the superorder Paracanthopterygii (Greenwood et.al., 1966). "Fishes,grouped together in this superorder represent a spiny finned radiation more or less comparable morphologically with that of the Acanthopterygii" (Greenwood et.al., 1966). Although they are morphologically comparable, evidence suggests that the two superorders, Acanthopterygii and Paracanthopterygi, are phylogenetically distinct, hence the common origin of the two remains unclear (Greenwood et al., 1966; Rosen, 1973). Gosline (1968) appears unsatisfied with the inclusion, by Greenwood et al., (1966), of the Gobiesociformes (order to which the gobiesocids belong) within the Paracanthopterygii, preferring to consider them as percoid derivatives and hence by implication within the Acanthopterygii. Fraser (1972) sheds little further light on where the Gobiesociformes should be placed, stressing mainly the equivocal relationships of this specialized order. Gosline (1970) suggests possible notothenioid origins for the Gobiesociformes, reinforcing his contention (Gosline, 1968) that the Gobiesociformes have percoid origins and hence again by implication belong to the Acanthopterygii and not the Paracanthopterygii.

Shifting the Gobiesociformes from one superorder to another has obvious phylogenetic significance. Placing the Gobiesociformes within the 
Paracanthopterygii (Greenwood et al., 1966) makes them widely divergent from the tripterygiids and clinids (Perciformes), whereas if placed within the Acanthopterygii (or along that line) as Gosline (1968, 1970) indirectly suggests, the Gobiesociformes are phylogenetically closer to the tripterygiids and clinids. Whatever their true origins the gobiesocids (clingfishes) constitute a specialized and advanced family of euteleosts (Greenwood et al., 1966; Rosen, 1973). The relationships of the genera and species within the Gobiesocidae are dealt with in detail by Briggs (1955). Included in his work is a review of the genera and species common to New Zealand and therefore further definition here is unnecessary.

The early literature e.g. Hutton (1904) recognizes seven tripterygiid species from New Zealand waters, although Waite (1913) accepts but three. That the tripterygiid fauna is much larger is suggested by Moreland (pers. comm.) who from a comprehensive knowledge of the fauna recognizes over 20 species. The nomenclature of the recognized New Zealand tripterygiids and clinids is curxently very confused, and therefore it is necessary to review in part the adult nomenclature. For this I rely on information from four main sources: collections by the present author of tripterygiid and clinid fish from rockpools and sublittoral zones in Port Nicholson and along the southern coast of Wellington; Mr J. Moreland (National Museum) who has the group under study; Mrs M. M. Darby (1966) who has reviewed the taxonomic status of Forsterygion varium, Tripterygion capito and the T. robustumT. nigripenne complex; and Mr D. Anderson, University of Auckland, who concurrently reviewed the common New zealand tripterygiids in conjunction with reproductive behavioural studies of some of the species. 
Blennioid fish with lateral line canals on suborbital ring and preopercle not covered by bone; first gill arch united to operculum by a membrane; no cirri on nape; scales usually ctenoid; pectoral rays usually branched; caudal rays always branched. Dorsal fin of three distinct portions, which may be connected at their bases - Hubbs (1952) Uppermost pectoral ray articulates with the scapula - Gosline (1968).

The New Zealand tripterygiids are abundant close inshore but many are also to be found in deeper water offshore. Four genera and 10 species are referred to here, but about 10-15 new forms remain undescribed. These are currently known only by such common names as the "Oblique-swimming blenny", "Yaldwyn's-blenny", "Blue-spotted" or "4-spotted blenny", "Yellow and Black blenny" and the "Scaly-headed blenny".

Forsterygion Whitley and Phillipps, 1940

First dorsal fin with V-VII spines; second dorsal with XIX-XXV; anal with about I-II, 25-3I; pectoral rays about 16 to 21 ; lateral ine anteriorly separated from base of second spiny dorsal by 5 or 6 scales - adapted from schultz et al., (1960).

Whitley and Phillipps (1940) designate Blennius varius (Forster in Bloch and Schneider, 1801) as the type-species of Forsterygion. They state that Cuvier and Valenciennes (1836), ..."figure this New Zealand blenny as Tripterygion nigripenne beside the typical Tripterygion (nasus)." Cuvier and Valenciennes (1836) describe T. nigripenne (figured as T. nigxipenne) and $\underline{T}$. varium $(=\underline{B}$. varius, not figured) as separate species. Whitley and Phillipps (1940) appear to have treated the two as synonyms. Subsequent studies (by the author) show Cuvier and Valenciennes (1836) to be correct and therefore T. nigripenne is figured correctly by them (i.e. as a species distinct from T. varium). Schultz et al. (1960) define Forsterygion more precisely than do Whitley and Phillipps (1940) and obviously use T. nigripenne as the type-species. This species has a lower number of spines in the dorsal fin than $T$. varium (B. varius). The confusion arises from the unfortunate synonymising of varium and nigripenne in the literature. Forsterygion is distinct from Tripterygion in having a greater number of spines in the dorsal fin, a proportionately larger head with a steeper profile and an overall greater size of the adults. 
Forsterygion varium (Forster, in Bloch and Schneider, 1801)

1801 Blennius varius Forster, in Bloch and Schneider, Systema Ichthyologiae, p.178.

1836 Tripterygion varium (Forster, in Bloch and Schneider, 1801), Valenciennes, in Cuvier and Valenciennes, Histoire Naturelle des Poissons, p.414.

1861 Tripterygium varium (Forster, in Bloch and Schneider, 1801), Gunther, Cat. fishes British Mus., 3:277.

1872 Trypterygium varium (Forster, in Bloch and Schneider, 1801), Hutton, Fishes of N.Z., p.33.

1877 Tripterygion nigripenne (non Valenciennes, in Cuvier and Valenciennes, 1836), Hutton, Trans. Proc. N.Z. Inst., 9:354.

1893 Tripterygion varium (Forster, in Bloch and Schneider, 1801),

Gill, Mem. natn. Acad. Sci., 6:119.

1940 Forsterygion varium (Forster, in Bloch and Schneider, 1801),

Whitley and Phillipps, Trans. Proc. R. Soc. N.Z., 69:236.

1968 Forsterygion varium (Forster, in Bloch and Schneider, 1801), Whitley, Aust. Zool., 15(1):80.

Forsterygion capito (Jenyns, 1842) •

1842 Tripterygion capito Jenyns, Zool. Voy. H.M.S. Beagle. Fish, (4): 94, pl.19, $1 \mathrm{fig}$.

1913 Tripterygion varium (non Forster, in Bloch and Schneider, 1801), waite, Rec. Cant. Mus., $2(1): 7$, pl.3.

?1926 Enneapterygius varius (non Forster, in Bloch and Schneider, 1301), Rendahl, Vidensk. Medd. Dansk nat. Foren., 81:10.

Forsterygion nigripenne (Valenciennes, in Cuvier and Valenciennes, 1836)

1836 Tripterygion nigripenne Valenciennes in Cuvier and Valenciennes, Histoire Natuxelle des Poissons, p.413, pl.338.

1861 Tripterygium nigripinne Valenciennes in Cuvier and Valenciennes, 1836; Gunther, Cat. fishes British Mus., 3:277.

1872 Trypterygium nigripenne Valenciennes in Cuvier and Valenciennes, 1836; Hutton, Fishes of N.Z., p.31.

?1873 Trypterygium nigripenne Valenciennes in Cuvier and Valenciennes, 1836; Hutton, Trans. Proc. N.Z. Inst., 5:263. 
1879 Trypterygium robustum Clarke, Trans. N.Z. Inst., 11:292, p1.15.

?1923 Tripterygion varium (non Forster, in Bloch and Schneider, 1801), McCulloch and Phillipps, Rec. Aust. Mus., 14:21.

I have placed capito and nigripenne in Forsterygion in company with F. varium, as they are all considerably larger and have more spines in the dorsal fin than the type-species, Tripterygion nasus Risso, 1826 (Mediterranean). Further examination of the known wide range of New Zealand tripterygiids may justify reallocation of one or more of the above species. The three species of Forsterygion described here are closely similar and considerable confusion has arisen regarding them. Forsterygion varium may be separated from F. capito and $F$. nigripenne by having more spines in the second dorsal fin (XXII-XXV C.f.XVIII-XXI), and a lateral line which finishes beneath the third dorsal fin. Another, as yet un-ramed, species of Forsterygion with virtually identical ray counts as $\mathrm{F}$. varium is found in the wellington area. It is distinct from the latter in having a consistent barred pigment pattern on the ventrolateral aspect of the head (mottled in F. varium) and the possession of an area of papillose scales which covers the dorsum of the head and passes further forward of the eyes.

Forsterygion capito is a smaller fish at maturity than F. nigripenne and is separated further by having a relatively steep, straight head profile and a higher number of tubercular scales in the anterior lateral line (21-23 c.f. 17-20). Members of the F. nigripenne series (see later) are immediately recognized by a very robust head region, the profile of which is always blunt and rounded. The nape is naked or covered by scales which are smaller than those on the rest of the body.

Because of the similarity of the above three species previous descriptions of any one species may have been derived from individuals of two or three closely related ones. There is therefore, in past literature, incomplete and possibly composite descriptions of fish which cannot easily be synonymised with any currently accepted species.

This problem is particularly relevant to $\mathrm{F}$. nigripenne. The exact status of this species is as yet unresolved. Moreland (pers. comm.) considers that there are two separate species, F. robustum (Clarke, 1879) a strictly marine form and F. nigripenne (Valenciennes in Cuvier and Valenciennes, 1836) the freshwater and estuarine form. The estuarine species differs primarily from the marine species in having a larger first dorsal fin and in the males an extended second dorsal fin that considerably overlaps the third. 
Darby (1966) considers only one species F. nigripenne with two subspecies, F. nigripenne nigripenne (freshwater and estuaries) and $\underline{F}$. nigripenne robustum (marine only). These are distinguished by differences in vertebral counts, third dorsal ray counts and variation in fin size. Darby recognizes only one species on the grounds that intermediate forms can be found with meristic counts that lie between the two extremes or subspecies. Anderson (pers. comm.) considers F. robustum (Clarke, 1879) to be "...a species restricted to exposed coasts where there is plenty of water movement and shelter in coralline turf etc." He regards F. nigripenne as a form that frequents sheltered reaches of shore, in areas where there are pools with fine sand, such as inlets, estuaries and harbours. Anderson maintains that within this species are probably two well defined forms which may warrant specific status after further study: an estuarine form and a sheltered marine type.

An analysis of meristic data of 35 specimens supports the contention of Darby (1966) and Anderson (pers. comm.) that F. nigripenne is a species with clinal variation between individuals in an estuarine habitat and those in a strictly marine but sheltered one (e.g. harbours or small inlets on exposed coasts). This polymorphism may be due to the different environmental conditions in which the eggs are incubated (see later in section on larval osteology). I can find nothing in Clarke's (1879) description to set F. robustum apart from $\mathrm{F}$. nigripenne and the former is therefore listed here as a synonym of the latter.

Gilloblennius Whitley and Phillipps, 1940

"This genus is distinguished by the very long lateral line, which is more primitive in structure than in other New Zealand blennies, in which it has atrophied. Dorsal spines three. Head pointed, its profile much less steep than in other three-fin Blennies" - Whitley and Phillipps (1940).

The two species are here referred to Gilloblennius: G. tripennis (Forster in Bloch and Schneider, 1801) and G. decemdigitatus (Clarke, 1879). The former is readily distinguished from the latter by the following meristic differences:

(i) G. tripennis D.III, XV-XVI, 13-16; A. I 22-24; Vert. 38-40.

(ii) G. decendigitatus D.III, XVII-IXX, 14-16; A. I 26-29; Vert. 42-44. 
Gilloblennius tripennis has a broader head than G. decemdigitatus and is typically more vividly coloured by reds, yellows and gxeens compared with the generally more uniform greys and blacks of the latter.

\section{Gilloblennius tripennis (Forster, in Bloch and Schneider, 1801)}

1801 Blennius tripennis, Forster, in Bloch and Schneider, systema Ichthyologiae, p.174.

1836 Tripterygion forsteri, Valenciennes, in Cuvier and Valenciennes, Histoire Naturelle des Poissons, p.415.

1861 Tripterygium forsteri, Valenciennes, in Cuvier and Valenciennes, 1836; Gunther, Cat. fishes British Mus., 3:278.

1872 Trypterygium forsteri, Valenciennes, in Cuvier and Valenciennes, 1836; Hutton, Fishes of N.Z., p.31.

1877 Trypterygiun forsteri, Valenciennes in Cuvier and Valenciennes, 1836; Hutton, Trans. Proc. N.Z. Inst., 9:354.

1893 Tripterygion tripenne (Forster, in Bloch and Schneider, 1801), Gill, Mem. natn. Acad. Sci., 6:100, 119.

1926 Enneapterygius tripennis (Forster, in Bloch and Schneider, 1801), Rendah1, Vidensk. Mędd. Dansk nat. Foren., 81:9.

Rendahl (1926) includes in his description of tripennis reference to decemdigitatus although he does not refer to it as such.

1940 Gilloblennius tripennis (Forster, in Bloch and Schneider, 1801), Whitley and Phillipps, Trans. Proc. R. Soc. N.Z., 69: 235-236. 1968 Gilloblennius tripennis (Forster, in Bloch and Schneider, 1801), Whitley, Aust. Zool., 15(1):80.

Gilloblennius decemdigitatus (c.larke, 1879)

1879 Trypterygium decemdigitatus Clarke, Trans. N.Z. Inst., 11:292, pl.15.

1893 Tripterygion decemdigitatum Clarke,1879; Gill, Mem. natn. Acad. Sci., $6: 119$.

1913 Tripterygion tripenne (non Forster, in Bloch and Schneider, 1801), Waite, Rec. Cant. Mus., $2(1): 3$, pl.1.

1968 Gilloblennius decemdigitatus (Clarke, 1879), Whitley, Aust. Zool., $15(1): 80$. 
"This genus is closely allied to Tripterygion Risso, but differs in the structure of its lateral line: this runs downwards from the shoulder to the middle of the side instead of extending backward parallel with the back, and there is no secondary series of incised scales posteriorly." MCCulloch and Waite, 1918.

Rosenblatt [1959, in Schultz et al., (1960)] has redefined the genus Helcogramma, and part of his definition appearing in Schultz et al., (1960) is as follows "... is restricted to those tripterygiid species possessing an axial lateral line composed of pored and unpored scales, one anal spine, palatine teeth and two pelvic rays connected by a membrane for a part of their length."

At present H. medium (Gunther, 1861) is the only New Zealand tripterygiid assigned to this genus. Evidence from larval studies shows the presence of at least one and possibly two other closely related species, the adults of which have not been formally described. The differences between these and $\underline{\mathrm{H} \text {. medium }}$ are subtle but consistent and suggest strongly that the adults, at least in terms of meristic data, are very similar. It is almost certain, therefore, that some of the descriptions, even the original (Ganther, 1861) refer to at least two very similar species. I have not found the adults of all the larval forms that I describe later, and without information from these I am unable to list with accuracy the synonyms of H. medium.

The larvae herein described as Helcogramma medium fit more closely with the first half of Glinther's (1861) description where he describes 7 specimens with 20-21 anal rays.

\section{Tripterygion Risso, 1826}

First dorsal fin with III, occasionally IV spines; second with X to XVIII; dorsal soft rays 6 to 15 ; anal I or II, 13 to 26 ; pectoral rays 14 to 18 ; lateral line represented by pores anteriorly and notched scales posteriorly; 2 or 3 scales between anterior lateral line and base of second dorsal - adapted from Schultz et al., (1960).

Tripterygion segmentatum McCulloch and Phillipps, 1923

1923 Tripterygion segmentatum McCulloch and Phillipps, Rec. Aust. Mus. , $14(1): 20, \mathrm{pl} .4$. 
1930 Notoclinops segmentatum (McCulloch and Phillipps, 1923), Whitley, Mem. Qld. Mus., 10:20.

1968 Notoclinops segmentatus (McCulloch and Phillipps, 1923) Whitley, Aust. Zool., 15(1):80.

Tripterygion bucknilli Griffin, 1926

1926 Tripterygion bucknilli Griffin, Trans. Proc. N.z. Inst., 56:544, pl.97.

1930 Notoclinops bucknilli (Griffin, 1926), Whitley, Mem. Qld. Mus., 10:20.

1968 Notoclinops bucknilli (Griffin, 1926), Whitley, Aust. Zool., 15(1):80.

Whitley (1930) assigns segmentatum and bucknilli to the new genus Notoclinops but gives no reasons for removing them from Tripterygion. I cannot find any distinguishing characteristics which justify this move and therefore return the latter species to Tripterygion.

Moreland (pers. comm.) and Anderson (pers. comm.) consider T. bucknilli as a synonym of $\mathrm{T}$. segmentatum as most of the distinguishing characters cited by Griffin (1926) are attributable to the fact that the holotype described by McCulloch and Phillipps (1923) was a juvenile (21 mm s.1.). Diagnostic characters such as unbranched pectoral and caudal rays and incomplete scalation relate directly to the young age of the holotype. Any further differences are attributed to natural variation as a result of differences in type locality, otago T. segmentatum and Bay of Plenty T. bucknilli. In the absence of information from a wide range of specimens, from the North and South Island, to substantiate a cline for these forms, I am reluctant to include $\underline{T}$. bucknilli as a synonym of $\underline{T}$. segmentatum. 
"Presence of scales; weak and flexible suborbital ring; covered lateral line canals on head; absence of branched rays in pectoral fins; absence of ctenii on scales. Jaw teeth conical in all members of this family. Most species have a patch of cirri over each anterior nostril and orbit" - Hubbs (1952).

Three genera and four species are referred to in this article. Ericentrus rubrus (Hutton 1872) and Cologrammus flavescens (Hutton, 1872) are ovoviviparous and belong to the tribe clinini (subfamily Clininae) (Penrith, 1969). Notoclinus fenestratus (Forster, in Bloch and Schneider, 1801) and N. compressus (Hutton, 1872) are oviparous and have been assigned to the subfamily Clininae by R. Rosenblatt (in Schultz et al., 1960). Notoclinus fenestratus and N. compressus should not, however, be included in the subfamily clininae as neither has a hook on the anterior edge of the cleithrum, small or embedded scales, scales with radii on all margins or unbranched rays in the caudal fin. These characters or rather the absence of them place these two species within the subfamily Labrisominae (Hubbs, 1952). The low number of pectoral rays (11) and lateral line scales (16-17) plus the presence of three.distinct dorsal fins necessitate the placing of $\underline{N}$. fenestratus and $\underline{N}$. compressus in a new tribe the Notoclinini.

Notoclinus Gill, 1893

"Differs from Tripterygion in having the spines of the first dorsal fin descendant, and the second fin not longer than the third. Pectoral rays less numerous and wholly undivided" - Waite, 1913.

Two species are described here, Notoclinus fenestratus (Forster, in Bloch and Schneider, 1801) and Notoclinus compressus (Hutton,1872). These species are readily separated by fin ray counts and relative sizes. Notoclinus fenestratus is a larger more robust species than $\mathrm{N}$. compressus and has the following meristic counts; D.IV, X-XI, 12-13, A. I,21-23, Vert. 39-40 compared with D.IV, X-XI, 10-11, A. I, 18-20, Vert. 35-36, for the latter. This distinction is further qualified by consistent differences between the larval stages. 
Notoclinus fenestratus (Forster, in Bloch and Schneider, 1801)

1801 Blennius fenestratus Forster, in Bloch and Schneider, Systema Ichthyologiae, p.173.

1836 Tripterygium fenestratum (Forster, in Bloch and Schneider, 1801), Valenciennes in Cuvier and Valenciennes, Histoire Naturelle des Poissons, 11:416.

1861 Tripterygium fenestratum (Forster, in Bloch and Schneider, 1801), Gunther, Cat. fishes British Mus., 3:278.

1872 Trypterygium fenestratum (Forster, in Bloch and Schneider, 1801), Hutton, Fishes of N.Z., p.32.

1877 Auchenopterus compressus (non Hutton, 1872), Hutton, Trans. Proc. N.z. Inst., 9:354.

1885 Trypterygium compressum (non Hutton, 1872), Arthur, Trans. N.Z. Inst., 17:168, pl.14, fig.6.

1890 Auchenopterus fenestratus (Forster, in Bloch and Schneider, 1801), Hutton, Trans. Proc. N.Z. Inst., 22:281.

1893 Notoclinus fenestratus (Forster, in Bloch and Schneider, 1801), Gill, Mern. natn. Acad. Sci., 6:100, 119, 124.

1913 Notoclinus fenestratus (Forșter, in Bloch and Schneider, 1801), Waite, Rec. Cant. Mus., $2(1): 9$, pl.4.

1968 Notoclinus fenestratus (Forster, in Bloch and Schneider, 1801), Whitley, Aust. Zool., 15(1):81.

Notoclinus compressus (Hutton, 1872)

1872 Trypterygium compressum, Hutton, Fishes of N.Z., p.32.

1873 Trypterygium compressum Hutton, 1872; Hutton, Trans. Proc. N.Z. $5: 263$, pl.xv, fig. 51 .

1876 Auchenopterus (Tripterygium) compressum Hutton, 1872; Hutton, Trans. Proc. N.Z. Inst., 8:214.

1926 Notoclinus fenestratus (non Forster, in Bloch and Schneider, 1801), Rendahl, Vidensk. Medd. Dansk nat. Foren., 81:12.

Hutton (1872) describes N. compressus as distinct from N. fenestratus, although his later works indicate that he was confused in dealing with both species. Hutton (1873) retains compressus but relegates fenestratus to being an "accidental variety" of F. nigripenne. Hutton (1876) places compressus in the genus Auchenopterus but later (1877), having resurrected 
fenestratus (from being an "accidental variety"), synonymises it with Auchenopterus (Notoclinus) compressus. Hutton (1890) accepting Forster's (1801) priority to the species title fenestratus, reverses the situation and lists compressus as a synonym of A. fenestratus. Gill (1893), places fenestratus in Notoclinus but retains compressus as a synonym. They are separated here on the grounds already described.

$$
\text { Ericentrus Gill, } 1893
$$

"Head and mouth small, body elongate, covered with small scales. Two dorsal fins not entirely separate, the second very long with a single posterior soft ray. Anal with two spines. Pectoral with undivided rays. Anterior portion of lateral line curved, composed of distinct scales."

- Waite, 1913.

Ericentrus rubrus (Hutton, 1872)

1872 Sticharium rubrum Hutton, Fishes of N.Z., p.33.

1873 Clinus rubrum (Hutton, 1872), Hutton, Trans. Proc. N.Z. Inst., $5: 264$, pl.9, fig. 53 .

1893 Ericentrus rubrus (Hutton, 1872), Gill, Mem. natn. Acad. Sci.', $6: 119,123$.

1913 Ericentrus rubrus (Hutton, 1872), Waite, Rec. Cant. Mus., 2(1):13, pl.5.

1927 Ophioclinus venusta Griffin, Trans. Proc. N.z. Inst., 58:149, fig.9

1968 Ericentrus ruber (Hutton, 1872), Whitley, Aust. Zool., 15(1):79.

Cologrammus Gill, 1893

"The Sticharium flavescens or Clinus flavescens of Hutton is differentiated from other types (and especially Exicentrus) by the form, more nearly even dorsal with a short-rayed portion (about 38 spines $t$ 6 rays), and imperfect lateral line; it may appropriately be designated as Cologrammus." - Gill 1893.

Cologrammus flavescens (Hutton, 1872)

1872 Sticharium flavescens Hutton, Fishes of N.z., p.33.

1873 Clinus flavescens (Hutton, 1872), Hutton, Trans. Proc. N.Z. Inst., $5: 264$, pl.15, fig. 54 . 
1893 Cologrammus flavescens (Hutton, 1872), Gill, Mem. natn. Acad. Sci. , $6: 119,124$.

1913 Cologxammus flavescens (Hutton, 1872), Waite, Rec. Cant. Mus., $2(1): 15$.

1968 Cologrammus flavescens (Hutton, 1872), Whitley, Aust. Zool., $15(1): 79$. 


\section{Family GOBIESOCIDAE}

The larval development of 9 clingfishes is described in this thesis. The adults of two species are as yet undescribed but the remaining 7 species are adequately described by Briggs (1955) and therefore require no elucidation here. The 7 are as follows:

Trachelochismus melobesia Phillipps, 1927

Trachelochismus pinnulatus (Forster, in Bloch and Schneider, 1801)

Diplocrepis puniceus (Richardson, 1846)

Gastrocyathus gracilis Briggs, 1955

Gastroscyphus hectoris (Gunther, 1876)

Dellichthys morelandi Briggs, 1955

Haplocylix littoreus (Forster in Bloch and Schneider, 1801) 
Larval, prejuvenile and adult ostology of Forsterygion varium

\author{
Introduction
}

Forsterygion varium (Forster, in Bloch and Schneider, 1801) is an endemic New Zealand tripterygiid which is present in harbours and shallow coastal waters. Previous works on $\mathrm{F}$. varium include:- its taxonomy (see list in review); parasites (Laird, 1951 and 1953); changes in metabolism (Morris, 1965); embryological and larval development (Ruck, in press a); and spawning behaviour and sexual dimorphism (Doak, 1972). The main purpose of the present study of its osteology was to provide information on the sequence and timing of the development of meristic structures such as spines, rays and vertebrae for use in identifying the larval stages of some New Zealand tripterygiids, clinids and gobiesocids.

Spines, rays and vertebrae are formed sequentially and the larval size at which these structures are laid down may be characteristic of a species and hence of value in identifying closely related species. The sequence of appearance and the position of fins and other bony structures have been used to identify larval stages of carangids (Ahlstrom and Ball, 1954; Berry, 1959; Aprieto, 1974; Miller and Sumida, 1974), myctophids (Moser and Ahlstrom, 1970, 1972, 1974), cyprinids (Balinsky, 1948; Richards and McBean, 1966), percids (Norden, 1961) and scorpaenids (Moser, 1972). However, what has not been used to any significant degree is the sequential development of, either spines and rays within any one $f i n$, or vertebrae. Because tripterygiids and clinids have slender bodies and long dorsal and anal fins it was thought that the sequential development of their bony structures might be exaggerated enough in time or spread out over enough of the larval life to make interspecific comparison of larvae easier. Preliminary observations of preserved larvae (not prepared for bone study) indicated this assumption to be correct. Accordingly it became necessary to document the time of appearance of bony structures, that is the larval size at which a structure began to ossify, and to see whether the uptake of alizarin (an indication of degree of ossification) varied with the condition (size and time in formalin) of the specimen. In addition it was necessary to find out the sequence of ossification, to determine when a ray, spine or vertebral complement was complete, and to see whether other 
less obvious bony features could be of value in characterizing larvae. It was hypothesised that the overall degree of ossification could be characteristic of a particular size larva of one species. However it was impossible to say how well a structure had ossified without knowing the condition in the mature adult. Therefore a detailed study of the adult osteology of $\mathrm{F}$. varium was undertaken, not only for the above purpose but also because the larval work appeared incomplete without some extension into the adult. The results of the osteology could also form a necessary base for a careful review of all the New Zealand tripterygiids, a task beyond the scope and aims of this thesis.

Initially it was intended to compare the larval and adult osteology of two tripterygiids, F. varium and an Helcogramma species (believed to be H. medium (Günther,1861) at the time the study was begun), with that of a clinid, Notoclinus compressus (Hutton, 1872). The comparison of three species was later considered unnecessary as the main purpose of this study was to supplement work on the identification of larval fishes. However the anterior skeleton of the Helcogramma species was already prepared and is included to show the relative position of the skull bones in tripterygiids.

Seven adults ranging in size from 55-80 mm s.1. and 16 larvae were cleared in $\mathrm{KOH}$ and then the bone was stained with Alizarin Red $\mathrm{S}$. The technique follows that of Hollister (1934) but was also used in combination with a trypsin method modified from Taylor (1967). Adults and larvae were partially cleared and stained in $\mathrm{KOH}$ and then transferred to trypsin solutions to prevent the disintegration of muscle tissue, and also to destain. Final clearing was in 50:50 glycerin and distilled water. Tap water was avoided as it tended to destain the bone, possibly due to the bleaching effects of chlorine. $\mathrm{KOH}$ at half the oxiginal strength was used to clear and stain the larvae. The cartilaginous elements of drawn specimens were not stained as they were clearly visible as translucent granular areas. Several adult skeletons were completely disarticulated by strong solutions of trypsin so that internal structures could be seen.

The degree to which alizarin is absorbed by the skeleton is taken as an indication of the amount of bone present at any particular larval stage. Only the best stained individuals were used to indicate the beginning of ossification of skeletal elements.

The terminology of the skull bones follows Harrington (1955), the axial and fin skeletons Hollister (1936) and Springer (1968), while that 
of the chondrocranium De Beer (1937). Drawings were made with the help of a camera lucida from a specimen $80 \mathrm{~mm}$ s.l. Unless otherwise stated the sizes of the adults and larvae are given as standard length.

\section{The adult skeleton}

In general the skeleton is well ossified with broad overlapping joints particularly of the bones forming the roof and floor of the neurocranium. There is very little firm interdigitation of adjacent bones. The elements are well ossified but quite transparent when stained showing the internal and external relief. Considerable time is required to distinguish the two aspects.

Neurocranium (Figs. $2 \& 3$ )

Ethroid: This is a small bone which lies in the sagittal plane supported by the cartilaginous ethmoid plate and nasal septum, but making no firm contact with any adjacent bones. It is thin and membranous and curls around the anterior face of the nagal septurn with the rear end open, in line with the splayed anterior vertical edge of the septal bone. Anteriorjy the ethmoid appears waisted broadening posterodorsally toward the anterior wings of the frontals, and expanding considerably anteroventrolaterally onto the ethmoid plate. There are two rounded points on the anteroventral edge.

Lateral Ethmoid: A broad membranous bone which is concave posteriorly around the anterior curvature of the orbit, broadly convex anterodorsally but concave anteroventrally to accomodate the olfactory organ. The dorsal lateral portion is simple and single layered but divides to form a two layered, complexly folded ventral portion. The two layers form a curved, almost tubular section which transversely crosses each lateral ethmoid. This tube (surrounding the lamina orbitonasalis) is complete ventrolaterally and on the medial edge of the large foramen for the olfactory tract. The layers radiate anteriorly, posteriorly and ventrally from the ventromedial edge of the tube to align with the posterolateral edge of the ethmoid, the anterolateral edge of the septal bone and the posterolateral margin of the prevomer, respectively. Each lateral. 
ethmoid is held firmly by the ethmoid plate, nasal septum and the lamina orbitonasalis, therefore making no direct contact with adjacent bones. Mid-ventrally there is a tight membranous fold which is filled with cartilage to form a round condyle. This articulates with a posterodorsal extension of the palatine. The lateral arm of the lateral ethmoid (lateral extension of tube section) attaches ventrally to the lachrymal.

Prevomer: This is a T-shaped bone with a tapered posterior shaft overlapping the anterior rostrum of the parasphenoid. The head is broad and hollowed ventrally, bearing about 30-35 sharp conical teeth on the anterior ventral margin. On the anterior border of the head is an almost vertical bony plate, slightly concave anteriorly, which is divided into three, a medial and two lateral sections. The dorsal surface of the prevomer is broadly attached to the ethmoid plate.

Parasphenoid: This is a long ventrally keeled bone, with a narrow anterior rostrum and a broad posterior plate. The posterior tapered extension of the prevomer slides into a similarly shaped groove on the ventral surface of the rostrum. Posteriorly the edges of this groove curl ventrally to firmly contain the prevomex. The rostrum forms the floor of the orbit. Level with the anterior margin of the prootic, wings extend abruptly dorsolaterally toward the anterolateral wings of the sphenotic. Fxom these dorsolateral points each lateral margin converges gradually posteromedially across the prootics to the median ventral surface of the exoccipital. There is a pronounced groove along the posterior, ventral mid-line which broadens into a deep notch on the posterior margin. The keel is deepest adjacent to the dorsolateral wings, level with the ventrolateral foramen for the carotid artery.

Basioccipital: A single strongly ossified bone with an expanded posterior circular condyle which articulates with the centrum of the first vertebra. Anteriorly it divides as two arms each grooved internally to form the postero-ventromedial floor of the otic bulla (contains the sagitta). Between these two arms is a shallow external groove which is covered by the posterior plate of the parasphenoid. The antero-ventrolateral edges meet the bulla portions of each prootic while the posterolateral edges are bordered by the antero-ventromedial margins of the exoccipitals. The arms are double 
membraned bones, while the central condyler portion is simply a thick margined tube.

Frontal: This bone is broad and thin posterodorsally and posterolaterally but narrows anteriorly above the orbit. The antero-ventrolateral margin is hollowed slightly encircling the dorsal and posterior curvature of the eye. Anteriorly this margin overlaps the posterodorsal extension of the lateral ethmoid. Above the margin and forming part of it is the bony tube which carries the supra-orbital canal. The anterior median part of this tube meets its opposite along the mid-line. The posterior medial edges of each frontal overlap broadly (either the right or the left may overlie its opposite). The anteriormost extension of the supraorbital tube swings away from the mid-line to line up with the nasal tube. There is a lateroventrally directed wing which tapers to a mid-lateral point overlapping the antero-dorsolateral surface of the sphenotic. The arched posterior edge of the frontal overlaps the anterior lateral wing of the supraoccipital and the anterior edge of the parietal. The posteriormost tip of each frontal may slide into a pocket on the parietal. A vertical fillet runs obliquely across the inner surface from the posterodorsal margin of the orbit towards the anterior lateral wing of the supraoccipital.

Parietal: This narrow bone borders antero-dorsomedially the deep otic depression which is formed by the 3 semicircular canals. The dorsal medial end is broad and double lobed while the anterior lobe lies partly beneath the frontal and overlies the anterolateral corner of the supraoccipital which interposes broadly between the parietals. The larger posterior lobe overlies the posterior lateral edge of the supraoccipital and the anterior dorsal surface of the epiotic. The entire anterior dorsolateral surface of the parietal is overlapped by the frontal. The antero-ventrolateral arm of the parietal tucks beneath the anterodorsal projection of the pterotic and part of the frontal, but overlies the dorsal surface of the sphenotic. Each parietal bends abruptly into the otic depression, forming a sharp ridge which rises to the dorsolateral surface and swings anteromedially in line with the commissural canal. Three curled membraneous bones lie above the parietal supporting the commissural sensory canal. The internal surface has a shallow groove which is continuous ventrally with another on the inside of the sphenotic. The anterior vertical semicircular canal follows this groove. 
Pterotic: This is a lateral bone forming the floor of the otic depression. It consists of an internal U-shaped tube with internally directed flared apertures. This tube carries the horizontal semicircular canal. An outer membranous bone (not tubular) meets the prootic antero-ventromedially, the exoccipital posteroventraliy and the epiotic medially in the otic depression. The anterodorsal extension of the pterotic overlaps the postexodorsal surface of the sphenotic and the lowest posterior corner of the parietal. A scroll-like ledge of bone extends anterolaterally from the pterotic to support part of the sensory canal. Beneath this ledge is a ventrolateral fossa which receives the posterior condyle of the hyomandibular. There is a posterolateral scalloped wing attached to the dilator operculi muscle.

Sphenotic: This is a relatively small bone on the anterolateral corner of the neurocranium. It is bordered and overlain by the frontal, parietal, pterotic and lateral edge of the prootic. A pointed portion (dermosphenotic) extends from the anterolateral margin to meet the posteriormost bone of the circumorbitals. Ventrally behind this is the deep, anterior hyomandibular fossa, half of which is also formed by the prootic. Directly above the fossa extending laterally from the sphenotic is a curled ledge of membranous bone. This is continuous with a similar structure on the pterotic and supports the sensory canal leading onto the circumorbitals. The anterior medial margin of the sphenotic meets the posterolateral edge of the pterosphenoid which is tucked under the orbital ledge of the frontal.

Prootic: Each complex prootic is bordered anterodorsally by the pterosphenoid, postero-dorsolaterally by the pterotic, dorsolaterally by the sphenotic, posteriorly by the exoccipital and posteromedially by the basioccipital. The ventral medial surface is broadly overlaid by the paraspenoid, while part of the posterior ventral surface is overlapped loosely by the opisthotic. The posterior ventrolateral surface of each prootic is rounded externally, grooved internally and bordered by inward projecting lamellae to form the antero-ventrolateral portion of the otic bulla. It is within this bulla formed by the prootic, exoccipital and basioccipital that the sacculus and its two otoliths, sagitta and tiny asteriscus, sit. Anterolateral to the bulla is a shallow and smaller internal depression carrying the utriculus with its flat, semicircular lapillus. 
The otoliths are relatively large and show faint growth rings in particular the large sagitta. Externally at the anterolateral corner is a stout bridge crossing a long, deep depression to meet the ventral edge of the fossa in which the anterior condyle of the hyomandibular articulates. Within the depression directly beneath the bridge lies a large anterior and a small posterior foramen through which the trigeminal and facial nerves pass. The anterior end of this depression opens towards the orbit, spreading laterally around the hyomandibular fossa, anterodorsally (broadly) to meet the pterosphenoid and medially onto a solid transverse bar which meets its opposite and the basisphenoid on the mid-line. This bar arises from the anterior medial surface of the prootic and has an anterolaterally directed foramen (presumably for the oculomotor nerve). The anterior ventrolateral surface of the prootic and the parasphenoid wings extend slightly further forward of the bar lobscuring its view ventrally). The ventromedial corner of the pterosphenoid is pocketed to receive the antero-dorsomedial margin of the prootic. The prootic, like most of the otic bones, is a double membraned structure which forms as perichondral lamellae on the otic capsule.

Epiotic: This is a small bone which forms the postero-dorsolateral corner of the neurocranium and carries part of the posterior vertical semicircular canal. It consists of a thin inner tube surrounded by an outer membranous bone. Antero-dorsomedially this tube is broadly flared and is directed in toward the brain. The ventral apexture is also flared, adjacent to the posterior opening of the pterotic tube, and in line with the shallow internal groove in the exoccipital. The outer membranous bone obscures the inner tube, meets the medial edge of the pterotic, the dorsolateral border of the exoccipital and the postero-ventrolateral margin of the supraoccipital, and slides under the parietal anterodorsally. The epiotic bears a postero-ventrolateral extension which is joined by a ligament to the post-temporal.

Opisthotic: This is a superficial bone overlying the ventral junction of the exoccipital, pterotic and prootic. It is a sub-triangular bone with a curled anteromedial edge which follows the lateral contour of the otic bulla. A small narrow ridge runs across the opisthotic towards the pterotic wing. It is easily removed from the rest of the neurocranium. 
Supraoccipital: A small median dorsal bone with its flat medial dorsal portion tucked beneath the frontals anteriorly and parietals laterally. The sloping posterior and medial areas are more ossified than the rest. The posteroventrolateral edges abutt the epiotics. The pointed posteroventral edge of the supraoccipital meets the antero-dorsomedial margins of the exoccipitals.

Exoccipital: An extremely complicated bone which forms the posterior ventral wall of the neurocranium. Externally each exoccipital meets its respective prootic anteriorly, basioccipital ventromedially, supraoccipital dorso-anteromedially, pterotic laterally, epiotic anterodorsolaterally and the opposite exoccipital medially. The short, lateral, vertical junction between the exoccipital and the pterotic is strongly interdigitated. Each exoccipital is a double layered (pexichondral) structure with a dorsolaterally directed internal groove (round externally) which is continuous with a similar groove in the epiotic. This carries the ventral part of the posterior vertical semicircular canal. At the lower end of this groove is a large ventrolaterally directed foramen for nerve $x$. Adjacent to the foramen is a small internal pocket carrying the asteriscus. This pocket broadens antero-ventrolaterally into the otic bulla carrying the sagitta. A small portion of the bulla is formed by the exoccipital while the rest is formed by the basioccipital and prootic. On the posteromedial internal border of each otic bulla is a solid septum which runs obliquely ventral from the middle of the exoccipital to attach firmly to the inner surface of the basioccipital. These two struts add considerable strength to this part of the neurocranium possibly to take the stress transmitted from the vertebral column. Projecting posteriorly from each exoccipital is a cone shaped condyle that articulates with equivalent surfaces of the first vertebra. A deep hollow curves down toward the foramen for nerve $\mathrm{x}$ between the rounded external portion of the semicircular canal and the dorsal medial ridge at the junction of the two exoccipitals. These and the basioccipital surround the posteriorly directed foramen magnum.

Basisphenoid: This bone is T-shaped when viewed dorsally, with the lateral edges of the $T$ firmaly attached to the transverse prootic bar. The shaft passes anteroventrally toward the parasphenoid broadening vertically and splitting to form an upper and a lower arm. The lower arm is blunt and paired laterally as weakly ossified membranes which meet the internal. 
mid-line of the parasphenoid. The dorsal arm tapers anteroventrally meeting no other bone.

Pterosphenoid: A small sub-triangular bone situated in the posterior lateral corner of the orbit. Dorsolaterally it is overlain by the frontal ledge, laterally it meets the medial orbital extension of the sphenotic and ventrally it contacts and partly underlies a broad orbital projection of the prootic. The ventral anterior surface is perforated by two oblique foramina which carry the trigeminalis and txochlearis nerves.

Septal bone: A single median bone consisting of a large compressed, curved tube with an anterior flared aperture which wraps around the posteriox edge of the nasal septum. The posterior ventral end of the tube is broady flared onto the trabecula communis directly above the parasphenoid rostrum. A rnembraneous septum arises in the sagittal plane from the dorsum of the tube. This septum passes between the eyes.

Circumorbital bones: There are 4 membranous bones around the ventral curvature of the eye. These extend from the lateral ethmoid to the sphenotic. The lachrymal is broad anteriorly and beaxs a posterodorsal projection which articulates with the arm of the lateral ethmoid. The bones decrease in size posteriorly, and the dorsal portion of each is curled to partly surround the infraorbital sensory canal. The anterodorsal margins slide beneath the preceeding bones of the series and are held firmly by connective tissue. The fourth circumorbital curls around the antero-ventrolateral wing of the sphenotic. The ventral margin of the second, third and fourth circumorbitals is curled laterally in places.

Sclerotic bones: The eye is bounded anteriorly and posteriorly by membranous concave schlerotic bones (not illustrated).

Nasal: Each nasal is a curved tubular bone which runs antero-ventrolaterally from the forward margins of the frontals. The apertures are both broadly flared.

Extrascapular: An L-shaped membranous bone with curled edges lying dorsolaterally above the otic depression. The dorsal arm of the $L$ carries 
the commissural sensory canal while the lower arm carries the lateral line proper. The antero-ventrolateral angle of the $I$ is open to receive the posterior extension of the pterotic sensory canal.

\section{Opercular Series (Fig. 4, no.2)}

This is a broad overlapping series whose elements are strongly ossified anteriorly and membranous posteriorly.

Preoperculum: A crescent shaped bone closely associated on the anterior curvature with the hyomandibular, symplectic and posterior quadrate. The entire length accommodates the preoperculomandibular sensory canal. Around the curvature runs an interrupted ossified tube with a medial surface spreading posteriorly and ventrally, and partly overlies the interoperculum. A rounded ledge or bone protrudes forward between the hyomandibular and the symplectic, while the anterior medial surface is notched to receive the interhyal which is fixed by a ligament.

Operculum: A triangular bone, concave medially and rounded on the posterodorsal margin. The long posteroventral margin overlaps the suboperculum and leads to a shaxp point ventrally. A strongly ossified condyle at the anterodorsal corner articulates firmly with an elongate condyle of the hyomandibular.

Suboperculum: An elongate bone curving beneath the posteroventral margin of the operculum and tapering posterodorsally to a thin weakly ossified slither. A narrow well ossified hook curves abruptly around the anterior and anteroventral edge of the operculum.

Interoperculum: An almost rhomboid plate which is considerably overlapped by the preoperculum. The dorsal portion is more strongly ossified than the rest and there is a poorly developed ball and socket joint between the posterodorsal edge and the adjacent suboperculum. The joint is held by ligament. 
Hyomandibular: This a Y-shaped tubular bone with membranous bone webbing the arms of the $\mathrm{Y}$. The two dorsal ends are capped with cartilage (remains of the hyosymplectic cartilage). These make firm contact with facets formed by the prootic, sphenotic and pterotic. The medial surface of the anterior dorsal arm is perforated by an elongate foramen opening into a canal which carries the hyomandibular branch of the facial nerve. This canal passes through the ventral arm of the $\mathrm{Y}$ and opens on the ventral lateral surface. The posteroventral margin curls laterally to make broad contact with the anterodorsal edge of the preoperculum. A tubular extension passes beneath the upper preoperculum from the dorsal posterior margin to meet the anterodorsal condyle of the operculum. This also has a solid cartilagenous plug to articulate on.

Symplectic: This is a tapered tubular bone, broadly flattened posterodorsally and spherical at the narrow anteroventral end. The latter passes deeply into a pocket in the quadrate firmly held by the unossified end of the hyosymplectic. The symplectic is joined to the hyomandibular by cartilage. Two membranous wings extend from the symplectic, one passing posteroventrally beneath the preoperculum and the other extending posteroventrolaterally.

Metapterygoid: A rectangular bone bordering the antero-ventromedial margin of the hyomandibular, the upper anterodorsal edge of the symplectic and the posterodorsomedial quadrate. The antero-dorsomedial corner is notched to receive the endopterygoid, while the opposite corner loosely overlaps the hyomandibular. The anteroventral sector is wedge shaped and double layered and formed by perichondral lamellae on the posterior palatopterygoquadrate.

Quadrate: A broad fan shaped bone with a large double headed anteroventral condyle articulating with the angular ("articular"). A long arm extends posterodorsally along the lower anterodorsal surface of the preoperculum. Between this and the quadrate proper is a deep pocket which receives the symplectic. The anterior vertical margin sits on a medial ledge along the posterior border of the ectopterygoid. A posteriorly directed flap of the ectopterygoids' lateral surface crosses the anterodorsal comer. 
Endopterygoid: A boomerang shaped membranous bone arching medially along the dorsal medial surfaces of the ectopterygoid and quadrate and notching posteriorly into the metapterygoid. The middle of the arch is ridged and bent vertically on the same plane as the other pterygoid bones.

Ectopterygoid: This is a narrow crescent shaped bone which is the most ossified in the series. The posterior vertical edge has a ledge to carry the quadrate. This ledge continues as a deep groove around the posterodorsal curvature to receive the anteroventral edge of the endopterygoid. The anterodorsal point passes into a tapered groove on the medial surface of the palatine. The tapered posteroventral point of the palatine slips into a pocket on the dorsal lateral surface of the ectopterygoid.

Palatine: Each palatine consists of a solid posteroventral portion and a curved antero-dorsolaterally directed arm. The lower portion is firmly attached to the ectopterygoid by ligament. There are several sharp cardiform teeth $(6+3$ small) protruding from the anterior medial surface, representing the dermopalatine. Above these is a bony rod which is directed posteroventrally toward the endopterygoid - an ossified vestage of the cartilagenous palatopterygoquadrate. Dorsally there is a deeply cupped mouth-like structure which articulates firmly with the ventral condyle of the lateral ethmoid. The antero-dorsolateral arm lies in a groove on the head of the maxillary.

\section{Lower Jaw (Fig.4, no's 1 \& la)}

Angular ("articular"): A well ossified bone with a broad shallow quadrate articulation surface bordered posteriorly by a hook-like posterodorsal extension. The angular rises steeply anterior to the point of articulation, and then falls gradually anteriorly into a deep pocket between the arms of the dentary. The main body of the angular is concave medially and convex laterally. Posteriorly within this concavity is a rod of bone (articular) which continues forward onto the dentary as meckel's cartilage. A ventral vertical plate bears the tube for the preoperculomandibular sensory canal. The medial surface of this plate is convex forming the 
inner wall of the tube. The lateral wall of the tube is incomplete. Directly beneath the articulation facet on the medial surface is a wide groove which snugly receives the retroarticular ("angular").

Retroarticular ("angular"): A small bone, with a dorsal conical and a ventral hoof-shaped portion. There is a ventral lateral ledge abutting the posteroventral edge of the angular.

Dentary: This is a stout bone with two posterior arms and a medially curved anterior shaft meeting its opposite at the midline. Along the dorsal edge are about 25 cardiform teeth. A further 24 much smaller teeth lie on a shelf of bone on the anterior inner curvature making a total of about 100 teeth on the lower jaws. The ventral area of the dentary including the lower posterior arm forms a tube carrying the anterior extension of the preoperculomandibular sensory canal. This tube is perforated by two large ventrolateral foramina and a ventromedial one. Posteriorly between the two arms is a deep pocket or tube which receives the angular. The meckel's cartilage also passes into this pocket deep within the dentary and is continuous with the anterior mentomeckelian ossification.

Upper Jaw (Fig.4, no.3)

Premaxillary: This is a relatively large bone with a long slender posterodorsally directed medial process. The solid ventral part is curved and bears on its anterior margin a row of about 20 pointed cardiform teeth. On the medial curvature is a pad of several hundred teeth which reduce in number towards the posteroventral end of the arm. The premaxillaries are separated by a thin layer of connective tissue. The posterodorsal ends of each long process are embedded in a mass of cartilage which slides along the anterodorsal groove of the frontals. Adjacent to the process is a posterodorsally directed portion loosely articulating with the head of the maxillary. Ventrally on each premaxillary is another posteriorly directed phlange. 
Maxillary (See shape on Helcogramma sp., Fig.1): An elongate bone with a solid ventro-posterolateral shaft (flared ventxally) joined by loose membranous connective tissue to the posterior margins of the premaxillary. At the anterodorsomedial end of the shaft is a solid spiralled head. This is large and bulbous posteriorly with the rounded surface articulating on the anterolateral concave surface of the prevomer. The head also has an anteromedial extension articulating on the smaller posterodorsally directed process of the premaxillary. Between the articulating surfaces of the vomex, maxillary and premaxillary are circular wads of cartilage which provide lubricated surfaces. The anterodorsal extension of the autopalatine sits in a deep groove on the posterodorsal part of the head.

\section{The laterosensory system}

The laterosensory pattern of $F$. varium is similar to that found in the blenniids (Springer, 1968). The canals are supported by continuous or interrupted ossified tubes and scroll-like ledges. The integumentary pores may open directly from the canals or via long, tubular extensions leading away from the main system.

The anteriormost opening of the supraorbital system is on the maxillary lip just forward of the nasal bone. The canal passes posteriorly through the tubular nasal, but before doing so gives off 7-8 small pores. These are arranged in a v-pattern around the edge of the large anterior aperture of the nasal bone. A single pore opens mid-dorsally on the tubular nasal, which itself has a broad posterior opening with two arms. The anterolateral arm carries part of the supraorbital canal to a large tubular pore opening directly in front of the posterior nostril. The posterior arm leads into the tubular ossification bordering the frontal. These two arms partly encircle the posterior tubular nostril. Passing from the supraorbital canal at the junction of the nasal, frontal and lateral ethmoid is an elongate tubular pore opening laterally on the fleshy circumorbital margin. This arrangement is typical of many pores around the eye. Scattered above the posterior opening of the nasal are 8-9 small pores opening directly to the surface. Nearly all of the cephalic canals are covered by these 
direct, irregularly arranged pores. The supraorbital canal passes around the lateral edge of the frontal onto the lateral surface of the sphenotic. A short transverse canal joins the two supraorbital canals at the narrow anterior junction of the frontals. This transverse connection passes through medial, narrow, elongate and opposing apertures in the tubular ossifications. Two medially placed pores lie above this connecting canal. The supraorbital canal has two rows of pores, one lateral series opening on the fleshy margin of the eye and another medial row with pores opening among the papillose scales. The supraorbital canal divides on the sphenotic to send one branch along the circumorbital bones to the lachrymal and the other onto the pterotic. The canal is supported on the pterotic and sphenotic by scroll-like folds which protrude laterally from each bone. Just before reaching the sphenotic the supraorbital canal sends a large branch across the frontal to a large lateral pore.

The infraorbital canal is partly surrounded by the circumorbital bones, moreso dorsally than ventrally. Two distinct rows of pores arise from this canal, an upper of small pores running along the lower margin of the eye and are partly surrounded by bone, and a lower of elongate tubular pores passing from the ventral margin of the canal to open above the cheek muscle. The longest and largest of these lower pores radiates anteroventrally across the surface of the lachrymal. The infraorbital canal terminates as a single large pore on the anterodorsal extension of the lachrymal adjacent to the anterior nostril. The pterotic canal, before passing onto the extrascapular, sends a branch down onto the preoperculum. This passes through an ossified tube on the angular, through another on the dentary, out a medial fenestra and across the intermandibular tissue to join with its equivalent from the other side. only portions of the canal on the preoperculur, angular and dentary are fully enclosed by bony tubes. This preoperculomandibular canal has two well defined rows of pores, an anterolateral and a posterolateral one. They are, however, interspersed by numerous smaller pores arising all over the canal. There are fewer pores along the angular and dentary and there is a single medial pore where the canals of each side join below the mandibles.

The pterotic canal divides on the extrascapular to form the commissural canal which meets its opposite above the supraoccipital and the lateral line proper. The latter passes posteriorly through a 
partly ossified tube on the post-temporal into tubular scales which run along the trunk following the dorsal contour to a point below the first ray of the third dorsal. From here it continues beneath a series of perforated scales along the mid-lateral line to the caudal joint. The commissural canal passes anterodorsally around the scale margin forming a posterior (relative to the canal) row of 10 tubular pores opening above the anterior myotome. Smaller tubular pores pass anteriorly above the parietals opening amongst the papillose scales. These pores along with tubular ones from the posterodorsolateral supraorbital form a uniform series above the frontals and parietals.

\section{Hyoid arch (Fig.5, no's 5 \& 5a)}

Interhyal: An elongate tubular bone with its cartilagenous ends firmly attached anterodorsally to the preoperculum and posteroventrally to the epihyal by ligaments.

Epihyal: A triangular bone strongly interdigitated on its anteroventral margin with the posterodorsal edge of the ceratohyal. The ventral lateral surface receives the heads of the two upper branchiostegals, which are the longest and broadest of the series. The antero-mesodorsal corner is hooked.

Ceratohyal: This is the longest bone of the arch. It has a broad posterodorsal blade and a tapered anteroventral shaft running beneath the ventral hypohyal. The anteroventral margin of the blade is notched to receive the two lower branchiostegals. The two remaining middle branchiostegals lie on the ventral latexal surface of the blade. On the antero-mesodorsal edge of the tubular shaft is an elongate membranous cone surrounding at its open end the posteroventral extension of the dorsal hypohyal.

Ventral hypohyal: This is a squat cone with a well ossified basal point which has a long posterodorsal extension. Between this extension and the cone is a deep pocket partly surrounding the ventral tubular shaft of the ceratohyal. The ventral hypohyals are joined medially by ligaments. 
Dorsal hypohyal: This is a fold of bone capping the medial mound of cartilage between the ventral hypohyal and the cone of the ceratohyal. The posteroventral surface is indented and overlies a small anterodorsomedial phlange of the ceratohyal. The posteroventral edge extends a short way into the cone mouth of the ceratohyal. The dorsomedial margin is ridged and curves abruptly onto the flattened anterodorsal surface.

Basihyal: An elongate tubular bone which is slightly wider anteriorly than posteriorly. The cartilage protrudes from each end.

Urohyal: This is a T-shaped bone with the dorsal horizontal surface broad anteriorly and tapered abruptly posteriorly. At right angles to this is a vertical posteriorly tapered plate which is divided anteriorly into two ventrolaterally directed phlanges. Between these phlanges is a sponge like network of bone. The urohyal is attached anteriorly to the posterior margins of the ventral hypohyals.

Branchiocrantum (Fig.5, no's $4 \& 4$ a)

The main elements are well ossified and tubular. Each tube is filled with cartilage which protxudes at each end to make contact with that of adjacent elements. The entire branchiocranium is surrounded by tissue of the anterior alimentary canal. The epibranchials and upper pharyngeals of each side approach each other at the dorsal mid-line and attach broadly via muscles to the ventral neurocranium.

Pharyngobranchial (upper pharyngeal): This is a broad bone which is concave dorsally and convex ventrally. The posterolateral end is a double tube structure facing the medial ends of the third and fourth epibranchials. A flattened tubular extension juts at right angles from the anterodorsal corner. The medial end of the second epibranchial fits closely into the inner angle of this extension. Because of its close association with the last 3 epibranchials it is likely the upper pharyngeal bone represents the second, third and fourth pharyngobranchials. Each upper pharyngeal bears about 25 curved conical teeth on its ventral 
surface (the largest are on the anteromedial edge).

Epibranchials: There are 4 curved tubular epibranchials. The first two are the longest having simple tubular medial ends and broad, phlanged lateral ends. The short third and fourth epibranchials have flared medial and lateral tubular ends. Only the posterior margins on these bear small phlanges.

Ceratobranchials: These axe elongate (except the fifth), slightly curved tubular bones. The medial and lateral ends of each are flared while the middle section is quite narrow. On the antero-ventrolateral and posteroventrolateral margins are two long keels aligning with two rows of ossified filaments supporting the gill lamellae. These filament rows pass ventrally beneath the hypobranchials and dorsally adjacent to the lateral ends of the epibranchials. On the medial curvature of each ceratobranchial is an anterior and a posterior row of gill rakers. The anterior row consists of 11 curved tooth-like elements. The posterior row is composed of 10 rakers consisting of inverted cones whose apexes are strongly denticulated. Each row is connected to the ceratobranchial by connective tissue. The fifth ceratobranchial is an elongate plate posterolaterally tapered and medially bluntly broad. The dorsal surface except the lateral end is covered in numerous curved, pointed teeth with the largest bordering the posteromedial margin. From the ventral antero-medial corner a tapered rod protrudes and plunges antero-ventrolaterally. The fifth ceratobranchials draw close together anteriorly on the ventral mid-line.

Hypobranchials: The first two are curved tubes with slightly flared ends. The posteromedial and anterolateral edges bear horizontal phlanges. The third hypobranchial is horn-shaped with its curved tapered end directed antero-ventrolaterally beneath the second hypobranchial. The open end is angled and faces the anteromedial projections of the third and fourth ceratobranchials.

Basibranchials: A single row is present along the ventral mid-line between the medial ends of the hypobranchials. The first is shaped like an arrow-head with curled lateral edges while the second is a short waisted tube half as long as the third which is elongate, narrow and posteriorly tapered. 


\title{
Pectoral and Pelvic girdles and fins
}

\author{
(Fig.5, no's 1, 2, 2a, $3 \& 3 a$ )
}

Posttemporal: Each has an anterodorsal arm attached by ligament to the epiotic and an anteroventral arm firmly attached to the posterolatexal surface of the pterotic. On the lateral surface level with the junction of the arms is an incomplete tube carrying the anterior lateral line before it passes into the tubular scales on the trunk. Beneath the posterior end of this tube is a solid, ventrally rounded condyle which fits into a shallow fossa on the supracleithrum.

Supracleithrum: This is a curved, laterally compressed bone with a lateral ledge bearing a dorsally directed fossa. The broad medial face overlaps and is firmly adhered to the dorsal blade of the cleithrum. The fossa receives the condyle of the posttemporal, and the anterodorsal extension slides beneath the latter.

Cleithrum: This is a broadly crescent-shaped bone which arches anteroventromedially to meet its opposite medially on the ventral mid-line. The cleithra are joined to the trailing edges of the urohyal by an elastic ligament. Each cleithrum is like a curved portion of "rightangled iron" with an almost vertical medial plate and a curved, laterally directed phlange. The lateral plate or phlange has a broad concave surface anteromedially. The internal angle of the right-angle is filled with a porous, bony fillet. The vertical plate broadens dorsally to receive the supracleithrum laterally and the postcleithrum medially. The anterodorsal corner is projected dorsally as a spine and there are secondarily thickened strips on the medial surface. The lateral plate tapers dorsally towards a broad foramen which opens into a deep sinus within the bony fillet. A similar foramen opens into a ventral sinus on the lateral surface above the pelvic girdle. An anterior marginal ridge which is continuous with the dorsal spine lies high on the medial surface. This ridge steps down to a broad posterior medial surface receiving the proximal ends of the scapula and coracoid. The marginal ridge broadens ventrally to lose its distinctiveness on the lower medial surface. Just above the cleithral symphasis on the inner aspect is a ventromedially directed foramen leading into the sinus within the porous bone. 
Scapula: The scapula is laterally compressed anteroventrally with the ventral lateral surface firmly attached to the cleithrum. The distal end is strongly ossified and has 3 definite condyles: an anterodorsal one ligamentously attached to the posterior margin of the cleithrum, a posterodorsal one with a cartilagenous cap (part of the main tubular structure of the scapula) directed toward the dorsal pectoral rays, and a postero-ventromedial condyle loosely attached by muscle to the dorsomedial margin of the upper radial. The centre of the scapula is perforated by an elongate foramen. The cartilagenous core is continuous ventrally with that of the coracoid.

Coracoid: An L-shaped bone with a posteromedially directed spur. The main part is a flattened membranous tube which is narrow anteroventrally where it attaches to the outer surface of the cleithrum. The lateral surface of the flared dorsal end is broadly adhered to the medial surface of the cleithrum. The posterior margins of the scapula and coracoid articulate with the radials.

Radials: The lower 3 are horizontal "hour-glass" tubes surrounding cartilage. Posteriorly they articulate with the pectoral rays and anteriorly with the posterior margins of the scapula and coracoid. The articulating ends are cartilagenous. The area between the flared extremeities of the radials is filled with vertical membranous bone. The lower membrane of the ventral radial is curled posterolaterally receiving on its anterodorsal surface a triangular muscle, which runs forward to the lateral cleithral plate. The dorsal radial has a well ossified proximal end fused to the posteroventral margin of the scapula. The dorsal vertical web is absent and the distal end is fan-shaped like the other radials. The rays are all segmented distally and gradually decrease in length dorsally and ventrally to the thirteenth. The uppermost 3-4 are the unbranched, the next 6-7 branched, followed by 7 thickened and unbranched rays. The proximal ends are divided and have strong lateral and medial processes for the attachment of adductor and abductor muscles.

Postcleithra: Two laterally flattened, elongate bones which pass obliquely beneath the proximal ends of the pectoral rays. The dorsal one is broad anterodorsally where it joins the cleithrum and tapers 
posteroventrally to meet the thin "boomerang" shaped ventral postcleithrum.

Pelvic girdle: Each pelvis is a laterally convex bone (deeply concave medially) with a thin, external, dorsoposteriorly directed phlange on the antero-dorsolateral margin. Directly beneath this phlange is a curved posterolaterally tapered tube which gives strength to this part of the pelvis. Extending posteriorly from this tube is the thin dorsolateral "roof" curving to meet the strongly ossified posterodorsolateral edge. The antero-ventrolateral and postero-ventrolateral "floor" is irregular and incomplete. In the vertical medial plane there is a pitted plate, broad posterodorsally and tapered unevenly anteroventrally. The medial surface of this plate, the edge of the "roof" and the open ends of the tube are firmly joined to their opposites on the neighbouring pelvis. The complete unit is tightly wedged between the ventral cleithra. Most of the contact with each cleithrum is along the external surface of the phlange. The posterolateral point of each pelvis is strongly ossified and has condyles and depressions articulating with the pelvic spine and the two proximally divided rays. The spine is short, tapered and considerably dwarfed by the long, curved, segmented rays. The medial ray is the longesst. There is a prominent, triangular dorsolateral and a ventromedial process on the spine. The latter process passes well beneath the rays. The upper arm of the medial ray has a dorsolateral process and the lower a medial one. Adjacent to the inner ray is a small posteromedially directed spur which is attached to the pelvis. This is possibly a vestige of the third ray found in blenniids (Springer, 1968).

\section{Vertebrae and Unpaired fins (Fig's 6 \& 7)}

There are 3 dorsal fins, the anterior two have spines, and the last soft segmented rays. The spines vary in length and each articulates via a ring-joint with an elongate pterygiophore. The pterygiophore is a curved tubular rod dorsally enclosing a foramen. The posterodorsal end loosely articulates with a recess in the anterior dorsal margin of the next pterygiophore. The ventral end of the tubular rod is extended anteriorly and posteriorly by broad membranous webs. The pterygiophores of the first dorsal fin are smaller and narrower than those of the second, 
although the very first has an anteriorly expanded web lying close to the posterodorsal roof of the neurocranium. There are 6-7 spines in the first and 23-25 in the second dorsal fin. The proximal pterygiophores of the third dorsal and anal rays each consist of a single curved tube with a flared distal and a tapered proximal end. The anterior and posterior margins bear broad web-like extensions and the anterior web is reduced in the anal fin.

The flared end of the proximal pterygiophore is smoothed by a cartilagenous condyle articulating with the anterior end of the distal pterygiophore. An anterodorsal process (anteroventral is the anal fin) meets the posterior, rounded end of the preceeding distal pterygiophore. The latter is a short curved cartilage which is ossified laterally as a paired structure. The rays are divided to produce lateral arms with medial processes running beneath the ventral concavity of each distal pterygiophore. The last proximal pterygiophore of the third dorsal and anal fin has an additional posteroventral and posterodorsal process respectively. Also the distal articulating surfaces are doubled and rounded laterally to receive the last two reduced rays of each fin. There is no distal pterygiophore. It is likely that the last proximal pterygiophore of each fin is formed by the fusion of two previously separate ones. The third dorsal has 14-15 rays and the anal fin 26-30.

The anal rays are preceeded by two spines. The anterior one articulates via a ring-joint with a slender pterygiophore. The posterior spine also articulates via a ring joint, but this is with an anteroventral process from the proximal pterygiophore of the first anal ray.

There are 48-51 vertebrae including the urostyle. Sixteen to 17 of these are precaudal without haemal spines. The centra are amphicoelus, constricted medially with definite longitudinal struts and bearing a notochordal canal. The anterior 5 centra are shorter than the rest and are more firmly articulated with each other. The centra of the first 3 vertebrae have posteroventrally directed processes overlapping the next succeeding centrum. These processes are not homologous to neural, haemal, pre- or postzygapophyses (Springer, 1968). The neural prezygapophyses of the first vertebra are enlarged anteriorly and articulate with the exoccipital condyles. The next 4 vertebrae also have well developed but more slender neural prezygapophyses which articulate anteromedially with the postero-dorsolateral surface of the 
preceeding centrum. The remaining vertebrae have zygapophyses which draw near to but do not contact those of adjacent centra. Articulation is restricted to the anterior and posterior surfaces of the centra. The fifth to eleventh vertebrae have ventrolaterally directed parapophyses bearing the third to ninth pleural ribs. The first two pleural ribs articulate with small fossa on the ventral lateral surfaces of the third and fourth neural arches. The parapophyses of the twelfth to sixteenth centra are directed ventrally and are joined medially by parapophysial stays. The ventral extensions of the parapophyses swing laterally to carry the remaining 5 pleural ribs which decrease in length posteriorly. The first two epipleurals are attached loosely to sockets in the neural arches of the two anterior vertebrae. The third to twelfth have elongate heads fastened to the trailing edges of the first 10 pleural ribs. The thirteenth to seventeenth epipleurals meet the haemal arches of adjacent vertebrae, while the remaining 8 smaller ones lie on the septum between the hypaxial and epaxial muscles. All vertebrae except the first and last have neural spines and arches which extend posterodorsally to overlap the pterygiophores of the dorsal fins. The second neural spine is poorly defined. Each broad neural arch from the sixth to the penultimate vertebra is perforated by a large, lateral foramen. The haemal spine is present on all vertebrae from the eighteenth onwards overlapping the proximal pterygiophores of the anal fin. The haemal arches are narrow on all but the last 8 vertebrae where they are broad in keeping with the neural spines. These and the solid neural arches and spines support the slender caudal peduncle. The penultimate vertebra has a well developed haemal spine which helps support the 7-8 procurrent rays. The neural spine is reduced extending posteriorly between an anterodorsal groove in the urostyle. This groove also receives the anteroventral ends of the epurals.

The 9-10 dorsal procurrent rays are supported by the posterodorsal extensions of the two epurals and the neural spine of the antipenultimate vertebra. The broad dorsal hypural plate is fused to the posteroventral margin of the urostyle and carries one unbranched and 6 branched rays. The ventral hypural plate is firmly attached to the lower posteroventral. curve of the urostyle and carries 5 branched and two unbranched rays. It is perforated on the anterior lateral surface by foramina which receive the branches of the caudal artery. Each caudal ray is segmented and 
divided (except procurrent rays) with arms which overlap the posterior borders of the hypurals.

\section{Discussion - adult skeleton}

Before relating the osteology of $\mathrm{F}$. varium to that of other blennioid fishes it is necessary to outline current views on the status of families within the superfamily Blennioidae. Hubbs (1952) recognizes four families, the Pterygocephalidae (fossils only), Tripterygiidae, Clinidae and Blenniidae within the superfamily Blennioidae (Blenniicae) of the suborder Blennioidei. Stephens (1963, 1970) includes another family, the Chaenopsidae, recognized earlier as a subfamily (Chaenopsinae) within the Blenniidae (Hubbs, 1953) or the Clinidae (Springer, 1955). Although Springer (1964) doubts the family status of the Chaenopsidae he apparently continues to regard them as distinct from other blennioid families (Springer, 1968). Greenwood et al., (1966) and Gosline (1968) recognize the chaenopsidae and the other three families of living fishes within the Blennioidae.

Bohlke (1957), Robins and Randall (1965) and Bohlke and Chaplin (1968) do not recognize the Chaenopsidae as a family separate from the Clinidae. Bohlke and Robins (1974) go so far as to group the chaenopsids, the tripterygiids and the clinids within the one family Clinidae, and they recognize only two families the Clinidae and the Blenniidae. Springer (1966) even suggests that "...when these families are better known all will be synonymized with the Blennildae."

Despite the differing views outlined above and regardless of the taxa used it is possible to recognize groups of fishes which have "clinid", "chaenopsid", "tripterygiid" and "blenniid" characteristics indicating lines of divergence from some basal stock. When referring to their expanded family Clinidae Bohlke and Robins (1974) suggest that further research may reveal "...other lines of divergence within the larger unit that are similar in degree of specialization....to those that are 'tripterygiid' and 'chaenopsid'. For convenience in comparing the ostology of F. varium with that of other Blennioids, the somewhat less ambitious path of recognizing the four families Chaenopsidae, Clinidae, Tripterygiidae and Blenniidae is chosen in the present study. 
48.

That the Clinidae, Tripterygiidae, Blenniidae and Chaenopsidae have a close relationship has been substantiated by Hubbs (1952), Makushok (1958), Ford (1959 in Penrith, 1969), Gosline (1968), Stephens (1963) and Bohlke and Robins (1974). But "...the relationship of the "tropical" blennies (Blennioidae*) to any other fish group is by no means clear." (Gosline, 1968). Hubbs (1952) believes that the Blenniidae diverged from the main stalk of the superfamily earlier than either the Tripterygiidae or Clinidae, because all living blenniids are specialized in having high anteriorly located eyes and in being scaleless. Presumably a considerable amount of time (of separation) would be required to bring about these specializations (Penrith, 1969). The Clinidae and the Tripterygiidae share more generalized features than the Blenniidae, and the 'iripterygiidae are perhaps more primitive than the clinidae. The Chaenopsidae have characteristics essentially intermediate between those of the Clinidae and those of the Blenniidae (Penrith, 1969; Stephens, 1963) perhaps with a greater leaning toward the Clinidae (Stephens, 1970; Bohlke and Robins, 1974).

As previously stated the developmental and adult osteology of $\underline{F}$. varium was studied to supplement larval work and therefore no extensive ostological survey of the New Zealand tripterygiids and clinids has been attempted. The osteology of $F$. varium is, however, compared with that of the Clinidae, Chaenopsidae and Blennildae (from information contained within accounts given in the following paragraph). No chaenopsids and only two blenniids are found in New zealand waters, the latter two being restricted to Northern latitudes. In contrast the known New Zealand tripterygiids number over 20 species and the clinids four.

The osteology of the blenniids (excluding the New Zealand species) has been thoroughly covered by springer (1968) but except for superficial coverage in taxonomic studies (Hubbs, 1952; Bohlke, 1957; Stephens, 1963, 1970; Bohlke and Robins, 1974) the osteology of the chaenopsids, clinids and tripterygiids is at present unknown. A paper by R. H. Rosenblatt and J.S.Stephens $J r$, is soon to be published and one on chaenopsid osteology by V. G. Springer is being prepared (Springer, pers.comm.).

In the present study six specimens of adult clinids and eight of adult tripterygiids were prepared for bone study for comparison with F. varium: of the clinids, three were Ericentrus rubrus (Hutton, 1872) subfamily Clininae and three were Notoclinus compressus (Hutton, 1872) 
subfamily Labrisominae; of the tripterygjids, four were Helcogramma species [possibly H. medium (Gunther, 1861)] and four Forsterygion capito (Jenyns, 1842).

\section{The skull}

The tripterygiid and clinid skull is markedly less specialized than that found in the blenniids (Springer, 1968). The discussion which follows is one of comparison between the generalized osteology of the clinids and tripterygiids and the more specialized osteology of the blenniids, appreciating that the information on the former two families is almost entirely restricted to the few species mentioned above.

The clinid and tripterygiid neurocranium is narrower than that of the blenniids, particularly above the orbits where the frontals taper anteriorly. The prevomer is the anteriormost bone of the neurocranium. In the blenniids, however, the ethmoids typically extend forward of the prevomer, an arrangement which when coupled with the foreshortened jaw bones gives the typical blunt snout of the blenniids. The bones of the clinid and tripterygiid neurocranium are less firmly sutured than those in the blenniids, especially in the ethmoid region where the margins of the ethmoids and the prevomer merely touch in places, being supported and separated by the anterior chondrocranium. Where bones do overlap in the tripterygiids and clinids very little interdigitation takes place, an exception being between the exoccipital and the pterotic in the mature skull.

"In the blenniids the ascending wings of the parasphenoid reach the descending wings of the frontals and thus exclude the pterosphenoids from the orbital region" (Springer, 1968). In the clinids and the tripterygiids the wings of the parasphenoid are widely separated from those of the frontals, by interposing sphenotics and prootics. The sphenotic, parasphenoid and prootic bones are arranged to form a sharp angle to the posterolateral corner of the orbit, and the pterosphenoids are displaced anteriorly to form part of the posterior wall of the orbits (Fig.3 no.I). This arrangement places the articulation point of the hyomandibuj.ar vexy close to the orbit. The blenniid condition effectively elongates the neurocranium and separates the orbit from the hyomandibular, and allows the jaws to lie further back, almost directly beneath the oxbit. In 
both the Clininae and the Labrisominae (Clinidae) the ascending wings of the parasphenoid are reduced (i.e. as represented by E. rubrus and N. compressus respectively). In the tripterygiids the laterosensory organs and canals are supported by continuous or interrupted ossified tubes and scroll-like ledges especially on the sphenotic and the pterotic. The latexal extrascapular is a delicate tubular or scroll like bone (supporting the commissural sensory canal) which lies loosely supported by connective tissue within a depression bounded by the three semicircular canals of the parietal, pterotic and epiotic bones. The Labrisominae and the Clininae, as illustrated by $\underline{N}$. compressus and $\underline{E}$. rubrus respectively. exhibit a greater amount of bony protection for all their sensory canals than do the tripterygiids. In the blennids the sensory system is extensively protected by bone and the autogenous lateral extrascapulaxs are, in direct contrast with those of the tripterygiids and clinids, more closely associated with the parietal, pterotic and epiotic bones (Springer, 1968).

Smith and Bailey (1962) report that most teleosts, including the basal percoids, have six circumorbitals [more correctly infraorbitals (Springer, pers. comm.)]. Blennifids have two to five circumorbitals, including the dermosphenotic? (Springer, 1968, 1971, 1972). Springer (pers. comm.) states that primitive blenniids have five circumorbitals, Clinins five, labrisomins three to five, chaenopsids two to four and tripterygiids five, including in each case the dermosphenotic. The New zealand clinids and tripterygiids have four or five circunorbital bones. In the clinids and tripterygiids the last circumorbital makes contact with a short, curled projection (dexmosphenotic?) of the autosphenotic. In the blenniids the posteriormost circumorbital (referred to as the dermosphenotic) makes contact with the descending wing of the frontal, and may lie some distance from the sphenotic (Springer, 1968). The clinid circumorbital series is a weak one compared with that of either the blenniids or the tripterygiids. In the tripterygiids the bony canal of the infraorbital laterosensory system is poorly formed, existing only as small curled ledges in $\mathrm{F}$. varium and $\mathrm{F}$. capito.

All the clinids and tripterygiids examined have medial projections from the prootic bones which meet at the midline, and which join the meningost (postexior) portion of the basisphenoid. The prootic projection and the meningost form the roof of the posterior myodome. A similar 
condition is known in only two blenniid species, medial projections of the prootic being absent altogether in all others (Springer, 1968).

The parietals typically meet in the midline in the blenniids, whereas in the clinids and triptexygiids they are separated by the supraoccipital. The opisthotic (intercalar of springer) in the clinids and tripterygiids is a loosely held plate on the ventral aspect of the posterior neurocranium. In the blenniids the opisthotic is more firmly sutured to the pterotic, prootic and exoccipital bones in that region.

"The other families of the Blenniicae, generally considered less specialized than the Blenniidae, as well as many of the perciforms usually considered more primitive, have moderately well developed premaxillary ascending processes, teeth implanted on the bones of the jaws, no specialized posterior canines and dentaries that are joined by an even non-suturing joint" springer (1968). The clinids and the tripterygiids generally comply with the above except that the premaxillary ascending processes are very well developed, in fact more so than those in the Blenniidae. This factor gives the tripterygiids and the clinids a potentially more protrusible jaw system than that of the blenniids. Notoclinus compressus (Labrisominae) has peg-like teeth on the premaxillaries and dentaries, unlike the typical pointed teeth of other clinids and tripterygiids. Villiform teeth axe found on the palatines and the prevomer in the latter two families, but are never present on the palatines and only occasionally on the prevomer in the blenniids.

The suspensory elements of the blenniids appear to be more loosely arranged than those of the other three families, there being less of an integrated "shield" of elements protecting the gills and suspending the lower jaw. Furthermore in the blenniids the hyomandjbula and the opercular series lie a considerable distance from the orbit (Springer, 1968).

The New Zealand tripterygiids have an ossification on the cartilagenous interorbital septum. The origin and/or homologies of this bone is uncertain. The question that arises is whether this bone is a modified (beryciform) orbitosphenoid, considered lost in Perciformes (Greenwood et al., 1966), or whether it is a de novo ossification, the septal bone, as described by Springer and Freihofer (In press) for Pholidichthys. A description of its formation during larval development is included here in an attempt to clarify the situation. The anterior 
chondrocranium of F. varium (Fig. 11, no's 1 \& 2) has a small cartilagenous interorbital septum which extends posteroventrally fxom the nasal septum (upper portion) to make contact with the trabecular communis. Anteriorly this septum, while making contact with the nasal septum is also continuous with the taenia marginalis and the lamina orbitonasalis, the latter being perforated by the foramen olfactorium advehens. The olfactory nerve emerges from the anterior membxanous case around the brain, via the foramen olfactorium evehens at the upper margin of the interorbital septum and runs beside it, then through the foramen olfactorium advehens to the olfactory organ. The bone in dispute forms around this interorbital septum and complies essentially but not fully with De Beer's (1937) a description that the orbitosphenoid "..ossifies as perichondral lamellae on the interorbital septum and in the cartilage (preoptic root of the orbital cartilage) surrounding the foramen olfactorium evehens. The paired orbitosphenoids eventually fuse." In F. varium the bone does not form around the foramen olfactorium evehens but just in front, a situation that would only require a reduction in the amount of ossification of the orbitosphenoid, especially as this nerve foramen perforates the posterior margin of the orbitosphenoid normąlly. It appears that by definition the bone forming on the interorbital septum in tripterygiids could be called a modified orbitosphenoid. The bone, however, does not make contact with the pterosphenoid, as is typical of lower teleosts (Harrington, 1955) and the Beryciformes (Rosen, pers. comm.; Springer, pers. comm.), but instead it is widely separated from it by the membranous connective tissue supporting the brain. It does make contact, or at least closely approaches, the anterior arms of the frontals which form around the taenia marginalis, and in this complies with the situation in the Beryciformes.

The bone on the interorbital septum occupies a position similar to that of the rhinosphenoid of characids (Weitzman, 1962; Roberts, 1966, 1969). The rhinosphenoid possibly represents an anterior fxagmentation of the orbitosphenoid (Daget, 1964; Patterson, 1975). If the bone in F. varium is homologous to the rhinosphenoid then separation from the pterosphenoid would be accounted for by the loss of the true orbitosphenoid portion.

An argument against the presence of a true orbitosphenoid in F. varium is the phylogenetic relationship between the Beryciformes 
and the Perciformes of the Acanthopterygii, where the Beryciformes differ from the Perciformes by three main characteristics one of which is the possession of an orbitosphenoid (Greenwood et al., 1966). Rosen (pers. comm.) concludes "...that there is a transformation series of the character state orbitosphenoid which describes its gradual reduction and loss," from the Beryciformes through to the perciformes. Rosen (pers. comm.) also comments on the relation of the bone to the olfactory nerves in saying that "...an osteological novelty forming in the region of the olfactory sacs and extending backward in the orbit... would be expected to include these nerves." Furthermore the problem of homology is difficult because if there is going to be an ossification of cartilage in the interorbital region where else would it occur but in or around the cartilagenous interorbital septum? (Springer, pers.comm.). The fact that the rhinosphenoid of characids (in combination with an orbitosphenoid) is not present in any other family of fishes (Weitzman, 1962; Roberts, 1969) makes it unlikely that it is homologous with the bone in F. variun. The latter is almost certainly not a modified beryciform orbitosphenoid (above reasons) but appears to be an autogenous ossification, somewhat analogous to the rhinosphenoid in that it forms from two centres of ossification on a reduced anterior septum (interorbital) of cartilage (Bertmar, 1959). Its relative size, shape and position (with the exception of the orbitosphenoid) approaches that of the rhinosphenoid in the characid Acestrorhynchus (Roberts, 1969).

The bone which forms on the cartilagenous interorbital of $\mathrm{F}$. varium is here referred to as a septal bone (de novo ossification) as described by Springer and Freihofer (in press) for a similar ossification in Pholidichthys. Springer is at present using this information as evidence of a possible relationship between the Pholidichthyidae and the Tripterygiidae.

Vertebral column, unpaired and paired fins

The vertebral columns of the bleniids, clinids and tripterygiids are similar, with no neural spine on the first vertebra, a low spine on the second and a pronounced spine on the third. They all share the same tendency "..for the parapophyses of the middle precaudal vertebrae to develop wing-like processes that overlap and attach ligamentously to the next 
vertebra anteriorly" (Springer, 1968). The general pattern of epipleurals and pleurals is also similar for the three families, excepting minor variation between genera and species.

Gosline (1968) and Springer (1968) believe that the Acanthoclinidae or Opisthognathidae are the most primitive members of the blennioid stock. Springer uses the caudal fin of Acanthoclinus (from New Zealand material) "...as a basis for intexpretation of the blenniid caudal." The tripterygiid and clinid caudal fin, like that of the blenniids, is more specialized than that of Acanthoclinus, due to fusions or loss of some elements. The most primitive blenniid caudal has two epurals, an autogenous hypural five, a fused dorsal hypural plate and urostyle an autogenous ventral hypural plate and nine branched caudal rays. Other blenniids show variation on this one theme with fusion of the epurals and loss in some of hypural five (Springer, 1968). Individuals of the Clinidae and the Tripterygiidae also show variations that parallej those found in the Blenniidae. Notoclinus compressus (Labrisominae) has one epural (two have fused?) and a reduced hypural five. The ventral hypural may possess a ventral, posteroventrally directed spur. What this spur represents is uncertain. Helcogramma sp. and F. varium have two well developed and separate epurals but hypural five is not present. Notoclinus, Forsterygion and Helcogramma have branched and unbranched rays in their caudal fin, typical of the families (and subfamilies) they belong to. Ericentrus rubrus (Clininae) has two epurals and no hypural five. The epurals in some specimens are partially fused resembling closely the most specialized caudal type found within the Nemophidinae of the Blenniidae (Springer, 1968). Ericentrus rubrus has no branched rays in the caudal fin, another characteristic it shares with the Nemophidinae. The caudal fin of adult blenniids, chaenopsids, tripterygiids and clinids is typically rounded, although there are a few exceptions with notched and straight caudals. The number of segmented caudal rays in the clinids and tripterygiids falls within the range for blenniids of 10-15 (Stephens, 1963, 1970; Springer, 1968; Penrith, 1969; Bohlke and Robins, 1974).

The anal fins of the four families are similarly arranged, being long continuous series of rays preceded by I-II spines. The first spine is attached to its own pterygiophore (proximal) while the second is attached to the proximal pterygiophore of the first anal ray. It is realized that the pterygiophores in the blennioid dorsal and anal fins constitute the distal, medial and proximal radials with the latter two 
fused (Springer and Freihofer, in press; Springer, pers. comm.). However, the terminology proximal pterygiophore (= medial + proximal radials) and distal pterygiophore (= distal radial) is used here, when comparing blennioid families, in keeping with that used by springer (1968). In the tripterygiids, as in some blenniids (Springer, 1968), the terminal proximal anal pterygiophore supports two rays, and the posterior of the two rays is usually small and slender. This is not the case in Erubrus and N. compressus where there is no reduced extra ray on the terminal proximal pterygiophore. They do, however, lack the terminal paired distal anal pterygiophore and also the terminal paired distal dorsal pterygiophore. The absence of the distal pterygiophores and a single terminal ray present, is the more typical condition in the blenniids (Springer, 1968).

The dorsal fin exhibits a wide range of variation in the four families. There is a spinous portion followed by a rayed portion. The relative number of spines and rays in each portion is variable and the spinous portion is frequently subdivided into two parts (tripterygiids in particular). There are no gaps in the dorsal fins of either the blenniids or the chaenopsids that would indicate the loss of either a ray or spine (Stephens, 1963, 1970; Springer, 1968). The terminal spine in the blenniids may be reduced and the fin notched at this point to give a divided appearance to the dorsal fin. Furthermore different lengths of the spines and rays may add to the divided appearance of the dorsal fin, extreme examples of this being illustrated by the male chaenopsid Emblemaria diphyodontus (Stephens and Cervigon n.sp) (Stephens, 1970) and the blenniid Omobranchus aurosplendidus (Richardson, 1846) (Springer and Gomon, 1975). The dorsal fin in the clinids, especially the clininae, may also form a continuous series with the spines typically twice as numerous as the rays (Penrith, 1969). In Ericentrus rubrus there are no gaps and only one ray. This ray attaches to the proximal pterygiophore of the preceding spine (last in the spine series). In some clinids of the Clininae and frequently in the Labrisominae the first three or four spines maybe separated from the rest by a gap which has been formed by the loss of one or two spines (Hubbs, 1952; Penrith, 1969; Rosenblatt and Parr, 1969; Stephens and Springer, 1973). Notoclinus compressus (Labrisominae) has lost one or two terminal dorsal spines which are represented by one or two reduced proximal pterygiophores. The first dorsal portion of $\mathrm{N}$. compressus is composed of four spines. There is a 
large gap between the fourth and fifth spines suggesting the loss of at least two spines and their pterygiophores. There is also a gap between the third and fourth spine (within the first dorsal fin) which may indicate the loss of at least one spine and its pterygiophore. A similar and more extreme situation exists in the "clinid" Haptoclinus apectolophus (Bohlke and Robins, 1974).

The tripterygiids typically have three well defined dorsal fins, two anterior ones of spines only and a third of rays. Typically the divided dorsal fin is formed by the loss of spines and pterygiophores from between the first, second and sometimes third fins. The number of vertebrae between the second and third dorsal fin of the Helcogramma sp. suggests the loss of two pterygiophores and spines. In F. varium and F. capito, however, no such loss is apparent, except that there is no terminal spine on the proximal pterygiophore of the first ray as occurs in the blenniids (Springer, 1968). The spines in the first dorsal fin number less than those in the second and the total spine count always exceeds the ray count.

In the blenniids the coracoid is reduced and fused (synostosis) with the cleithrum (Springer, 1968). In the tripterygiids no fusion has occurred and the coracoid is well, developed. The scapula is reduced in the blenniids (Springer) and does not support the upper pectoral rays, whereas in the triptexygiids the scapula is elongate and its distal end lies level with the distal margins of the radials and characteristically supports the upper pectoral rays (Gosline, 1968). Notoclinus compressus has a pectoral condition similar to that in the tripterygiids. This situation, coupled with the three part dorsal fin (lost spines), castes some doubt about whether or not $\mathrm{N}$. compressus belongs to the Labrisominae, suggesting instead a possible affinity with the tripterygiids. A much more detailed study is necessary, howevex, before any conclusions about such a relationship can be made. Ericentrus rubrus, however, falls intermediate between the blennijd and tripterygiid condition. The scapula, although not as reduced as that in the Blenniidae, does not extend to the outer edge of the radials and therefore does not support any upper pectoral rays. A similar situation exists in the "clinid" Haptoclinus apectolophus (Bohlke and Robins, 1974). The coracoid in E. rubrus is not as reduced as that in the blenniids but it is similar in being partially fused to the cleithrum. Exicentrus rubrus has the anterior "hook" on the cleithrum, typical of the Clininae but not of the Labrisominae although Springer (1958) notes the presence of the cleithral hook in some specimens of some species of Malacoctenus (Labrisominae). The clinids, blenniids and chaenopsids have 
simple rays in the pectoral fin. In the tripterygiids the uppermost rays are characteristically branched. The dorsal pectoral ray of $\mathrm{N}$. compressus, which is supported by the scapula, is very reduced and visible only in stained specimens.

The clinids (Clininae and Labrisominae), chaenopsids and the tripterygiids have a radial formula of 2-1-1 which conforms with the generalized perciform condition of 2-1-1 (Regan, 1913). Springer (1968) suggests that most of the less specialized perciforms have a radial formula of 3-1-0 or 2-1-1 and therefore all chaenopsids, clinids and tripterygiids having a formula of 2-1-1 are less specialized than the blenniids (with $2-1-1,2-0-2$ or $1-0-3)$. Springer suggests that $2-1-1$ is the most primitive for blenniids, 2-0-2 is the most common and $1-0-3$ is the most specialized where the radials have "dropped" from the scapula onto the coracoids. The pelvic fins lie forward of the pectorals in the four families. The pelvic rays of the blenniids, chaenopsids and tripterygiids pass posterolaterally and act as simple struts on which the animal may rest. The range of movement of the rays is linited. In the clinids, however, the pelvics curve laterally from the body (Hubbs, 1952; Penrith, 1969; Rosenblatt and Parr, 1969) and are capable of a wide range of movements that allows a "walking" type of motion along the bottom. This greater movement is possible in the clinids ( $\mathrm{E}$. rubrus) because the pelvic rays and spine are less firmly fixed to the pelvic girdle than in the other three families where association is via a "ball and socket" type of joint.

"Nearly all tripterygiids are described as having $I, 2$ (pelvic fin rays) yet individuals of the West Atlantic species....have reduced third rays present" (Bohlke and Robins, 1974). Forsterygion varium has I,2 whereas Helcogramma has I, 3 with the third very reduced as described by Bohlke and Robins (1974). All chaenopsids have I, 3 pelvic fin elements (Stephens, 1963, 1970). Bohlke and Robins (1974) suggest that although clinids are described as having I,2 or I, 3 pelvic fin rays, "xe-examination of several species always recorded as having I,2 showed otherwise." Ericentrus rubrus very definitely has a I,2 ratio whereas $\mathrm{N}$. compressus has I, 3 pelvic-fin rays, with the third ray very reduced. Calliclinus (Labrisominae) has I,4 pelvic fin rays (Stephens and Springer, 1973). In the blenniids there is a range of $I, 2$ to I,4 (Springer, 1968).

It is generally accepted that the blenniids are more specialized than the chaenopsids, clinids and tripterygiids (Hubbs, 1952; Springer, 1968; Penrith, 1969). The tripterygiids and clinids are a mozaic of both 
58.

"primitive" and "advanced" features and according to Penrith (1969) it is difficult to say with any certainty which group is collectively more advanced than the other. The clinids possess several characteristics which are almost as specialized as those found in the blenniids. For example clinids have unbranched pectoral and caudal rays, partially fused coracoid and cleithrum (E. rubrus), covexed laterosensory canals, reduced scales (Clininae) and a scapula which typically does not support the uppermost pectoral rays. The tripterygiids also show some specialized features such as loss of spines from the dorsal fin, reduced pelvics (I,2 or I,3) compared with I,5 of the less specialized perciforms, and a depressed body. The tripterygiids and clinids share generalized features such as villiform teeth on jaws, vomer and palatines, no canines a typically perciform jaw apparatus and scales. However, "Aside fxom their specializations it would seem that the Tripterygiidae does show more unspecialized characters (branched pectoral-fin rays, ctenoid scales, scapula supporting pectoral-fin ray, sensory canal tube bones unroofed by bones etc.) than do the Clinidae" (Springer, pers. comm.). The chaenopsids on external features alone (Stephens, 1963, 1970; Penrith, 1969) share characteristics in common with both the blenniids and the clinids. Lack of information on chaenopsid osteology prevents further qualification of this point.

\section{The larval skeleton (Figs $8,9, \& 10$ )}

The cartilaginous skeleton: At hatching (5.85 mm s.1.) the anterior cartilaginous skeleton is present including the chondrocranium, jaws, hyoid arch and branchiocranium. The cartilaginous elements of the vertebral column and fins are laid down during later stages. The cartilaginous skeleton of the $16.5 \mathrm{~mm}$ larva has all the basic structures and is described here in detail. The cartilage is overlain with bone but continues to grow during later development.

Chondrocranium: The complete chondrocranium is broad posteriorly narrowing gradually anteriorly. The posterior dorso- and ventrolateral walls are formed by the otic capsules: regions of solid cartilage surrounding the auditory canals. The medial intrusions enclose the canals 
59.

but not the sacculus or utriculus. Anteromedially the walls of the otic capsules are contoured to fit around the mesencephalon and myelencephalon. The elongate head of the hyosymplectic fits into a ventral groove under the horizontal semicircular canal. The anterior part of this groove forms a distinct fossa which receives the forward condyle of the hyosymplectic head. Medial to the fossa is a broad bridge overlying the long depression leading into the foramina for nerves $V$ and VII. The contours on the otic capsule correspond to similar relief on the otic bones present in the adult. The floor between the otic capsules is broad, even, divided posteriorly by the notochord and perforated anteriorly by the hypophysial foramen. This foramen is obscured at $16.5 \mathrm{~mm}$ by the parasphenoid plate. The lateral arms of the trabecula cranii border the hypophysial foramen to join anteriorly as the trabecula communis, which continues forward as a narrow bar beneath the orbit and expands anteriorly as the ethmoid plate. The tectum synoticum forms the thin posterior roof of the chondrocranium between the otic capsules. The tectum cranii (roof above orbit) is represented by a narrow transverse epiphysial bar with a posterior, medial, poorly defined plate (taenia tecti medialis) which extends back toward the tectum synoticum. A similar plate (taenia tecti medialis anterior) passes forward to divide the epiphysial fenestra and broadening anteriorly to join the taenia marginalis laterally and the nasal septum anteriorly. These two medial plates arch gently over the mesencephalon and telencephalon. The nasal septum plunges obliquely to join the broad ethmoid plate, and consists of a solid sagittal ridge joined to the lamina orbitonasalis by a thin concave membranous cartilage. This shallow concavity (nasal fossa) continues ventrally with the dorsal surface of the ethmoid plate and accommodates the olfactory tube and organ. Olfactory nerves and blood vessels pass through a smal.1 foramen perforating the ventral end of the nasal fossa. The lamina orbitonasalis is anteriorly a well defined pillar extending laterally and ventrally beyond the lateral margins of the ethmoid plate. From here it rises postero-dorsolaterally around the curvature of the orbit as the taenia marginalis to join the epiphysial bar and finally meet the anterodorsolateral corner of each otic capsule. The brain is held in place by membranous connective tissue which lines the inner orbit and the area between the taenia marginalis. Between the maxillary bone and the anterolateral, scalloped face of the ethmoid plate is a small cylindrical rod. This is the preethmoid cartilage which 
acts as a lubricating surface between the maxillary and vomer. The posterior wall of the chondrocranium is formed by the externally grooved occipital arches which merge laterally and ventrally with the otic capsules. Each arch is perforated by a foramen for nerve $\mathrm{x}$, and joined dorsally with its opposite by the tectum posteriorus. The lateral commissure of the prootic passes transversely as a rod of cartilage behind the orbit. The interorbital septum is small and i.s joined to the nasal septum.

Suspensorium and hyoid arch: The hyosymplectic is broad dorsally and tapers anteroventrally to meet the posteroventral margin of the quadrate. The medial and lateral surfaces are smooth and perforated to carry the hyomandibular nerve. The dorsal head articulates with the ventral lateral surface of the otic capsule. The palatopterygoquadrate forms the ventral curvature of the orbit. The fan-shaped quadrate leads into the long pterygoid and palatine shaft on its anterodorsal corner while the concave medial surface of the palatine meets the lateral margin of the ethmoid plate. An elongate drop-shaped 'labial' cartilage surrounds the two posterodorsal premaxillary processes acting as a sliding surface between the premaxillaries and the nasal septum. The ventral condyle of the quadrate fits firmly into a posterior dorsal socket on meckel's cartilage, from which a pronounced coronoid process rises anterodorsally. The main bar of meckel's cartilage curves medially to meet its neighbour at the mid-line.

The hyoid arch is complete. The ceratohyal is long, broad in the middle and tapered ejther end. Antero-ventromedially the ceratohyal meets the hypohyal which is a blunt anteriorly tapered cartilage with a curved posterolateral and a medial spur. The latter tucks beneath the anterior basibranchial. The basihyal is a single elongate rod overlapping the anterior tip of the first copula which is an elongate medial rod ossified later in three parts. The second copula is a small disc of cartilage sitting on the end of the first. It does not ossify latex. The remaining elements of the branchiocranium are present as solid cartilages resembling the tubular portions of those in the adult. 
Vertebral column, girdles and fins: Each pelvis is concave medially, convex laterally and tapered posteriorly. The blunt anterior end meets its neighbour medially and the two lie wedged between the cleithra. The coracoid and scapula are continuous forming a narrow inverted T-shaped plate. Four rectangular radials border the curved posterior margin of the scapula. The notochord is a cellular tube surrounded by a thickened sheath and is constricted slightly between centra. Each bony centrum is clasped on its anterior dorsolateral and ventrolateral surfaces by broad arch bases (basidorsals and basiventrals). These are directed posterodorsally and posteroventrally to semi-enclose the spinal cord and blood vessels respectively. The supradorsal and infrahaemal cartilages (spines) are laterally compressed and slightly wider than their respective arch bases. The cartilagenous arch bases and spines are joined only by ossified sections of the arches, although in $16.5 \mathrm{~mm}$ larvae they are close together and appear joined. The last vertebrae are without arch bases, although their associated spines are well developed. The first 17 centra bear tiny arch bases at points where later parapophyses arise. The neural spines (supradorsal cartilages) of the first 11 centra are absent although their arch bases are present. The arches of these centra remain unjoined through to the late prejuvenile stage. It is apparent that the cartilagenous precursors of bony axial elements are formed just prior to the onset of ossification. The centra of the vertebral column are ossified before all the arch bases and spines have formed. The earliest complete vertebral count is obtained from Alizarin stained specimens, not from those stained for cartilage by Victoria Blue or Toluidine.

The hypurals and epurals are present as separate elements resembling those of the adult. The ventral hypural is perforated anteriorly by two foramina, the anterior of which later disappears. The pterygiophores supporting the longest rays of the dorsal. and anal fins are clearly defined. The distal pterygiophores are similar to those in the adult while the proximal pterygiophores are narrow posterodorsally and laterally compressed anteroventrally. At $27 \mathrm{~mm}$ these follow the tubular pattern of those in the adult as do the pterygiophores supporting the spines of the dorsal fins. A summary of the cartilagenous development is as follows:

(1) Primary upper and lower jaws, hyoid arch, branchiocranium and chondrocranium arise before any axial elements. 
(2) Hypurals, infrahaemals and supradorsals form in the tail first and develop sequentially anteriorly.

(3) Arch bases arise anteriorly and extend slowly posteriorly.

(4) All pterygiophores develop posteriorly first moving gradually anteriorly. The pterygiophores of the rays are formed before those of the spines.

These basic trends are modified by the tendency for the largest elements (or those supporting the largest) in a series, to form before the rest. For example the posterior pterygiophores of the anal fin appear before the anterior ones. However, the last rays but 4 are the longest in the adult and the pterygiophores of these develop before any posterior or anterior to them.

\section{Ossification of the larval skeleton}

\section{Neurocranium}

Ethmoid: This bone even in the adult is weakly ossified and appears in prejuveniles over $27 \mathrm{~mm}$ as a superficial ossification of the anterodorsal surface of the nasal septum. From here it spreads gradually around the septur and ventrally toward the ethmoid plate.

Lateral ethmoid: This develops as a perichondral lamella on the orbitonasalis $(27 \mathrm{~mm})$ later forming the tubular section of the adult bone. Radiating laterally from the lamella is a curved more strongly ossified dermal plate, representing the prefrontal (Harrington, 1955). There is very little endochondral ossification of the lateral ethmoids. Instead in specimens larger than $27 \mathrm{~mm}$ the primary ossification spreads ventromedially onto the ethmoid plate leaving the ventral surface unossified.

Prevomer: This appears at $16.5 \mathrm{~mm}$ as a T-shaped bone closely associated with the ethmoid plate but not as a perichondral ossification of the latter. The anterolateral corners curve ventrally following the ventral relief of the ethmoid plate. At $27 \mathrm{~mm}$ the anterior margins begin to grow around the anterolateral. surface of the ethmoid plate. Teeth also begin 
developing on the anterior ventral margin. As early as $16.5 \mathrm{~mm}$ the posterior shaft is firmly attached to the rostrum of the parasphenoid.

Parasphenoid: At $10.5 \mathrm{~mm}$ it is visible as a thin anteriorly and posteriorly tapered plate running from beneath the ethmoid plate to the anterior tip of the notochord. The posterior portion soon broadens $(16.5 \mathrm{~mm})$ and extends posteriorly beneath the basioccipital. The ascending wings develop as slender transverse processes directed toward the pterosphenoid $(27 \mathrm{~mm})$. Behind these are small notches which become deeper as the processes and lateral margins spread. The notches are eventually surrounded to form foramina carrying the carotid arteries into the parabasal canal beneath the lateral commissure (prootic bar). At $28.7 \mathrm{~mm}$ the parasphenoid overlaps the medial ventxal surfaces of the prootics.

Basioccipital: This is one of the first bones to ossify, and is present in the $10.5 \mathrm{~mm}$ larva as a tapered tube surrounding the anterior tip of the notochord. At $16.5 \mathrm{~mm}$ two separate areas of the chondrocranial floor begin to ossify internally and externally. These lie anterolateral to the anterior tip of the notochord and constitute the anterior arms of the basioccipital. They are joined medially by a ventrally concave plate and extend posteriorly to join the anterolateral margins of the notochordal ossification.

Frontal: Initially each frontal is laid down above the taenia marginalis $(24 \mathrm{~mm})$ and spreads laterally, anteriorly and posteriorly, to meet its opposite at the mid-line and the parietals posteriorly. The plate directly above the mesencephalon is the last to ossify and is complete at $28.7 \mathrm{~mm}$. The addition of superficial relief (secondary thickening) is a process which continues throughout the adult life. The dorsal and medial grooves of the tubular sensory canals form as fine curled bones along a line medial and parallel to the plate above the taenia marginalis.

Parietals: This dermal bone is present at $16.5 \mathrm{~mm}$ as an extremely faint membrane overlying the dorsolateral surface of the anterior semi-circular canal. From here it grows mostly on its anterodorsal and posteroventral edges beneath the frontals, over the supraoccipitals and down into the 
otic depression. The postparietals appear at $28.7 \mathrm{~mm}$ as curled membranes loosely attached to the dorsal surface of the parietals.

The otic bones: These all arise at approximately the same stage $(24 \mathrm{~mm})$ and are formed in a similar manner. Each begins as perichondral lamellae about the canals of the auditory capsule. Lamellae are laid dow on the external and internal surfaces, giving a double membraned structure as found in the adult. Individual bones first form as isolated units (middle zones of the fully developed bones) and gradually spread to meet adjacent bones. Except for a few the borders between the otic bones are separated by cartilage of the auditory capsule. The dermal portions associated and finally fused with the otic bones form at about the same time. These include the dermosphenotic which develops on the anteroventrolateral projection of the auditory capsule and the curled portions of the sphenotic and pterotic which support the sensory canals. The superficial ossification of the epjotic receiving the posttemporal does not form till later in development (juveniles). The prootics ossify on their posterior areas first, spreading forwaxd and across the bridge to the anterior hyomandibular fossa. The last portion of the prootic to ossify is the lateral commissure. The opisthotic which is merely a superficial plate is laid down at $27 \mathrm{~mm}$ beneath the inner curvature of the horizontal semi-circular canal.

Supraoccipital: This is a perichondral bone which forms around the anterior tapered part of the tectum synoticum and the narrow posterior projection of the taenia tecti medialis. Initially $(27 \mathrm{~mm})$ it is composed of an elongate tubular rostrum which broadens posteriorly as a semi-circular plate. As development advances it spreads laterally beneath adjacent bones.

Exoccipital: These bones develop as perichondral ossifications of the lateral occipital arches in larvae between 12 and $14 \mathrm{~mm}$. This produces the double membraned bone typical of that in the adult. An endochondral ossification is directed ventromedially fxom the inner lamella toward the basioccipital to form the rigid strut. By $24 \mathrm{~mm}$ the lamellae have grown anterodorsally, laterally and anteroventrally to surround the 
foramen for nerve $x$. Future development is simply the gradual expansion of the internal and external lamellae to meet adjacent bones.

Basisphenoid: The basisphenoid is barely visible at $28.7 \mathrm{~mm}$. It consists of a weak anterior vertical plate dividing anteroventrally about the interorbital septum. The posterior transverse bar forms against the anterior edge of the lateral comrissure. The anterior plate and the posterior bar join later.

Pterosphenoid: This is first visible as faint perichondral ossifications of the posterior taenia marginalis on the posterior inner curvature of the orbit $(28.7 \mathrm{~mm})$. These ossifications expand to meet the prootics, frontals and sphenotics and surround the tiny foramina for the trochlearis and trigeminalis nerves.

Septal bone: This forms as perichondral lamellae around the interorbital septum. The septum is barely visible during the early stages and ossification of the lateral surfaces does not begin until $28.7 \mathrm{~mm}$. The anteroventral margins of the lamellae meet at the mid-line. The posterodorsal margins also meet but along a greater length, eventually rising posterodorsally as a median plate between the eyes.

Circumorbitals, nasals and extrascapulars: These develop as simple membranous plates which gradually spread and curl at their margins. The lachrymals are visible in 16 man larvae followed by the fourth, the second and finally the third circumorbitals. The nasals are present at $24 \mathrm{~mm}$ and the extrascapulars at $27 \mathrm{~mm}$.

Opercular bones: These form as wide plates at $16.5 \mathrm{~mm}$ with strongly ossified anterior margins. At $28.7 \mathrm{~mm}$ the elements overlap considerably and the preoperculum is curled anteriorly to carry the sensory canal. 


\section{Suspensorium}

Symplectic and hyomandibular: These appear in $16.5 \mathrm{~mm}$ larvae with the symplectic slightly more ossified, particularly ventrally. They form as separate perichondral ossifications of the hyosymplectic, and each resembles that of the adult at $28.7 \mathrm{~mm}$. The dorsal articulating surfaces of the hyosymplectic are retained in the adult.

Quadrate, metapterygoid and autopalatine: The palatine and the quadrate are distinctly visible at $24 \mathrm{~mm}$, but arise as faint plates during earlier stages $(16.5 \mathrm{~mm})$. The metapterygoid appears much later at $27 \mathrm{~mm}$. All develop as perichondral lamellae on the palatopterygoquadrate. The ossification of the palatine, in particular the anterior arm lags behind that of the other two bones, and the dermopalatines are not added till at least $28.7 \mathrm{~mm}$. The quadrate ossifies ventrally first and gradually spreads dorsally until at $24 \mathrm{~mm}$ the middle portion of the ligament between it and the preoperculum is ossified. This ossified ligament has joined the quadrate by $28.7 \mathrm{~mm}$.

Ecto- and endopterygoid: While dermal in origin these bones are laid down close to the surface of the palatopterygaquadrate and arise at the same time $(16.5 \mathrm{~mm})$. They form close to each other on the anteroventral and posterodorsal margins of the palatopterygoid shaft, eventual.y broadening to meet adjacent bones.

Hyoid arch: The lower part of the ceratohyal is ossified at $10.5 \mathrm{~mm}$ followed by the upper part (epihyal) at $16.5 \mathrm{~mm}$. The ventral hypohyal surrounds the ventral curvature of the hypohyal cartilage and develops a posteriorly directed spur by $27 \mathrm{~mm}$. The dorsal hypohyal and interhyal ossify much later at $28.7 \mathrm{~mm}$. All of the hyoid elements except the urohyal are perichondral ossifications which form curved or tubular structures. The urohyal is present as a medial vertical anteroventrally divided plate at $24 \mathrm{~mm}$ and the anteroventral edge of the 'epihyal' interdigitates slightly with the posterodorsal margin of the ceratohyal by $28.7 \mathrm{~mm}$. The dermal branchiostegals appear first as slender laterally compressed and curved rods $(10.5 \mathrm{~mm})$. 
Branchiocranium: All the elements of the branchiocranium are perichondral ossifications except the upper and lower pharyngeal tooth plates, which are dermal in origin. These tooth plates and the ceratobranchials are faintly visible in $10.5 \mathrm{~mm}$ larvae. Small areas of bony material develop in the middle of individual elements gradually spreading to form the basic tubular adult pattern. The basi-, hypo- and epibranchials start to form at $27 \mathrm{~mm}$ and the basibranchials develop as 3 ossified centres along the first copula. The anterior dorsal gill rakers of the first ceratobranchial are quite bony at $27 \mathrm{~mm}$ whereas the gill rakers on the other arches are present only as translucent cartilages.

Secondary upper jaw: The maxillary arises first $(7-8 \mathrm{~mm})$ as a thin curved bone running from a point antexolateral to the ethrnoid plate down to overlap the lateral surface of the dentary. The antero-dorsomedial head is twisted and the ventral shank is broadened slightly and by $19 \mathrm{~mm}$ the typical adult features are acquired. At $8-9$ mon the premaxillary is present as a very thin bone arching around the anteroventral edge of the maxillary. The dorsal medial end has a small posterodorsally directed process and by $10.5 \mathrm{~mm}$ the postero-ventrolateral axm has broadened to meet the maxillary ventrally. Small teeth line the anteroventral margin. The typical adult features are present at $19 \mathrm{~mm}$.

Lower jaw: The dentary, angular and retroarticular appear with the maxillary $(7-8 \mathrm{~mm})$. The dentary is a curved dermal plate adhering to the external margin of meckel's cartilage. Posteriorly it broadens and is notched to receive the tapered anterior end of the wedge-shaped angular. The articular is not ossified. There is a faint indication of the retroarticular which is the perichondral ossification of the posteroventral corner of meckel's cartilage. By 16.5 the anterior curvature of the dentary appears to have enclosed the anterior part of meckel's cartilage as the mentomeckelian cartilage. The posterior notch is deeper and the dorsal area of the dentary is a broad, vertical, membranous plate bearing several small teeth. By $24 \mathrm{~mm}$ the basic arrangement and shape of the bones in the lower jaw approaches that of the adult. The sensory tubes of the dentary and angular are not formed until at least $28.7 \mathrm{~mm}$. At $24 \mathrm{~mm}$ the articular portion of meckel's cartilage begins to ossify. 
Pectoral and pelvic girdles: The cleithrum (along with the jaws) is the first bone to develop. It forms as a narrow twisted rod (strongly ossified) which passes obliquely in front of the pectoral fin to meet its neighbour on the ventral mid-line. At $13.5 \mathrm{~mm}$ the supracleithrum and posttemporal are present linking the dorsal blade of the cleithrum to the cranium. There is initially no ventral arm on the posttemporal and the sensory tube is not present until $28.7 \mathrm{~mm}$. Plate like extensions develop in various planes about the basic cleithral rod and by $24 \mathrm{~mm}$ it is a simplified replica of that in the adult. At this size the radials are barely ossified while the rays of the pelvic fin have begun to ossify along their margins. Arching around the posterior margins of the radials are 19 (full adult complement) divided and well ossified rays. The dorsal postcleithrum is present as an oblique streak beneath the fleshy pectoral lobe. At $28.7 \mathrm{~mm}$ the coracoid and scapula are lightly and evenly ossified and the tubular waisted radials bear vertical membranous plates. The rays have medial and latexal processes and the ventral postcleithrum is visible. Each half of the entire pelvis is bony receiving the full complement of divided rays and spines on its posterolateral corner.

Vertebral column and median fins: "The centra develop directly from skeletogenous tissue surrounding the notochord. Each forms as a faint ring growing rapidly laterally and becoming strongly ossified. Those directly above the hindgut develop first $(12 \mathrm{~mm}$ ) followed by the anterior and posterior ones. Between 13 and $14 \mathrm{~mm}$ a few centra remain unossified but by $16.5 \mathrm{~mm}$ the series is complete, although the posterior 2-3 may be barely visible. The neural and haemal arches and spines begin to ossify at $16.5 \mathrm{~mm}$. Bone is laid down on the arch bases and gradually spreads to join the supradorsal and infrahaemal spines respectively. The arches on the first centra to form are the first to ossify, moving slowly anteriorly and posteriorly. At $24 \mathrm{~mm}$ all arch bases are ossified including the reduced anterior parapophyses. The first 5 neural arches are not joined.

At $11.5 \mathrm{~mm}$ there are 14 well defined caudal rays the middle 5 having two segments and are the longest. There are faint perichondral lamellae either side of the hypurals and a band of bone surrounding the anterior end of the urostyle. The full complement of segmented rays is present along with 3 dorsal and ventral procurrent rays in $16.5 \mathrm{~mm}$ larvae. The middle 5 rays are the longest with 5 segments each while the others have 
only 3. The ventral hypural is perforated by two foramina on each side, the posterodorsal one later occluding. All the procurrent rays are visible by $27 \mathrm{~mm}$ and the dorsal and ventral segmented rays have overtaken the middle ones to produce a notched posterior margin. There are 7-9 segments in each and overgrowth of the dorsal and ventral rays is caused by elongation of the basal segments.

The spines and rays of the dorsal and anal fins ossify long before their respective pterygiophore elements. Posterior rays are typically the first formed although modified by the tendency for the largest to arise first. Dorsal and anal rays begin to ossify between 13 and $14 \mathrm{~mm}$ moving gradually anteriorly and reaching their full complement by $16.5 \mathrm{~mm}$. Cartilagenous pterygiophores are present at the same time as the spines and rays ossify and are therefore helpful (when stained) to reinforce meristic counts. The central tubular region of each proximal pterygiophore forms as a perichondral lamella $(27 \mathrm{~mm})$ but it is not until $33.5 \mathrm{~mm}$ that the distal pterygiophore ossifies. All the spines of the first and second dorsal fin are present at 27 and $24 \mathrm{~mm}$ respectively. An anterodorsal projection of each spine pterygiophore is the first part to ossify (27 mm) gradually spreading ventrally. This spur projects anterodorsally through the aperture between the ventral arm of the spines.

\section{Discussion - larval skeleton}

The literature dealing with the development of cartilaginous and bony skeletons of fishes is extensive and therefore only a few of the more important references are mentioned here. Information on the origin of the skeleton is perhaps of greatest value in revealing homologous and analogous structures which are of so much importance in phylogenetic studies [Hubbs, 1926; Norman, 1926; Gregory, 1933; De Beer, 1937; Holmgren, 1943; Goodrich, 1958 (and numerous references therein); Bertmar, 1959; Kapoor, 1970; Bjerring, 1972]. While almost all studies on skeletal development have some phylogenetic importance, some are more directly concerned with other aspects such as establishing the significance of meristic characters for use in adult taxonomy (Lindsey, 1954; Tyler, 1963; Garside, 1966; Ciechomski and Weiss de Vigo, 1971; Fahy, 1972) or for identifying the larval stages of fishes (see references cited in the introduction). Works of a more genexal nature on skeletal 
ontogeny include those by Runyan (1961), Mansueti (1963), Peckham (1963), Benzie (1968b), Woolcott et al., (1968), Potthoff (1974), Houde and Swanson (1975), and Jollie (1975).

The cartilaginous skeleton of F. varium is laid down in a pattern typical of that found in other teleosts (for the latter see Norman, 1926; De Beer, 1937; Holmgren, 1943; Goodrich, 1958; Bertmar, 1959; Jollie, 1975). The tropibasic chondrocranium is sparingly endowed with cartilage, much of which persists in an expanded form into the adult. The persistance of the chondrocranium accounts for the comparatively minor degree of interdigitation of bony elements in the adult skull [c.f. the neurocranium of hexagramids (Quast, 1965) and percids (Osse, 1969)] so that much of the structural strength is provided by cartilage. This is particularly the case in the ethmoidal region. The brain is 'protected' more by membranous connective tissue than by cartilage, especially in the orbital region and dorsally. The chondrocranium exhibits, as in other advanced teleosts (Springer, 1968; Gosline, 1968, 1970; Osse, 1969), a strong "tendency for the anterior part of the cranial cavity to be narrowed and reduced from below upwards by the enlarging orbits and the formation of the septum, and for the bulk of the brain to be pushed backwards... the orbital walls meeting in the middle line." (Goodrich, 1958). The interorbital septum (orbital. walls meeting in the mid-line), around which the septal bone later forms (see adult skeleton), is small and situated well forward between the orbits. In the $16.5 \mathrm{~mm}$ specimen it is barely visible whereas in the adult the relative size of the interorbital septurn (septal bone) increases, thus illustrating well the trend described above by Goodrich.

The ossification of the various elements in the skeleton also follows a pattern typical of teleosts in general (Harrington, 1955; Goodrich, 1958; Moser, 1972; Jollie, 1975). Obviously, however, the tripterygiid skeleton lacks some of the bony elements normally found in lower teleosts, such as the preethmoid, supxamaxillary, supraorbital and medial rostral etc. (Jollie, 1975).

The prevomer in $\mathrm{F}$. varium does not show the bilateral orjgin which i.s illustrated in the less advanced esocids (Jollie, 1975). It develops, instead, from a single centre below the ethmoid plate.

Jollie (1975) prefers to call the pterotic the intertemporosupratemporal, on the grounds that these two dermal elements "have fused in actinopterygians and extended perichondrally and endoperichondrally into the neurocranium." In F. varium the dermal and chondral portions of 
the pterotic arise at about the same time (dermal poxtions a little latex in some specimens) but are distinctly separate entities arising from different anlagen. The chondral portion also arises from two ossification centres, one internal to the cartilagenous horizontal semicircular canal and the other external. The adult pterotic forming from these centres resembles a tube within a tube, with the inner "tube bone" making no contact with the outer. The intertemporo-supratemporal canal units are represented by an anterior curled ledge of bone and a posterior wing (supratemporal?) to which the dilator operculi muscle attaches. The separate developments of these pterotic bones and the reduced state of the canal units in F. varium do not support directly Jollie's origin of the pterotic.

In $F$. varium the quadrate has a long arm which extends posterodorsally along the lower anterodorsal surface of the preoperculum. Between this and the quadrate body is a deep pocket which receives the symplectic. The quadrate arm is also present in other teleosts including characids (Weitzman, 1954, 1962; Roberts, 1966, 1969), cyprinids (Harrington, 1955), gobiesocids (Gosline, 1970), percids (Osse, 1969), sciaenids (Topp and Cole, 1968), blenniids (Springer, 1968) and many others. In F. varium the quadrate arm ossifies separately from the main body of the quadrate on what appears to be ligamentous connection between the quadrate and the preoperculum. Jollie (1975) describes this ossification in the esocids as the quadratojugal and calls the combined bone in the adult the quadratojugo-quadrate. That the element arises separately from the quadrate is quite clear in $\mathbf{F}$. varium. Its identity as a quadratojugal, however, is no more certain.

The upper jaw elements develop in a manner typical of Perciformes in general (Berry, 1964) whexe the maxillary develops first followed by the premaxillary. The latter arises anterodorsal to the naxillary and then extends posteriad to exclude the maxillary from the gape. Such a sequence in development supports the view that the upper jaws in advanced teleosts apparently "...reflect or pass through the primitive condition of upper jaw structure during their larval period." (Berry, 1964). The premaxillary develops quickly, however, and very soon acquires small functional teeth important for early predatory life.

Meristic structures of Forsterygion nigripenne (Cuviex and Valenciennes, 1836) exhibit a cline from an estuarine form through to a 
sheltered marine form. The vertebrae, anal rays and dorsal rays of the estuarine form are consistently lower in number than those of the marine form. These meristic differences may be accounted for by differences in the environment in which the eggs of the two forms develop. Several workers have established a correlation between (certain) meristic counts and environmental factors such as carbon dioxide content, oxygen content, light intensity and duration, temperature, salinity and productivity of the water in which egg and larval development take place. The higher the temperature, for example, the smaller the number of vertebrae formed on the average (Lindsey, 1954; Lindsey and Ali, 1965; Garside, 1966; Ciechomski and Weiss de Vigo, 1971; Fahy, 1972). The higher temperatures have the effect of speeding up development and thus lowering the number of vertebrae formed. A decrease in salinity may have the same effect as raising the temperature, during embryological development (Schmidt in Garside, 1966). The final number of vertebrae is fixed pxior to hatching (Taning, 1946) during somite formation (Fahy, 1972). Johnson and Barnett (1975) have shown that eggs and larvae from areas of low productivity are on the average larger than those from axeas of high productivity, and suggest that this is an apparent compromise between death by starvation or by predation, both of which are related to size. The mean values of vertebral counts etc., are lowest in the smaller eggs and larvae; the converse is also true. Johnson and Barnett consider that "...egg size and hence size at hatching are genetically determined features reflecting adaptation to productivity conditions."

The average temperature of estuarine water in New Zealand even during winter and spring when F. nigripenne spawns is likely to be slightly higher (but locally there is no evidence available to support this) than that encountered on the southern coast of the North Island where cold currents pass through cook strait. What is certain is that estuarine water will have on the average a lower salinity than strictly marine water. Higher average temperatures and lower salinity and hence shorter pexiods of development may well account for the lower vextebral counts of the estuarine form of F. nigripenne. Because there is a 1:1 ratio of rays to vertebrae in the tripterygiids (Cosline, 1968) a reduced vertebral number, especially in the caudal region (Ciechomski and Weiss de Vigo, 1971), may well alter dorsal and anal ray counts also. If so this would account for the consistently lower ray counts in the estuarine form of $\mathrm{F}$. nigripenne. 
This situation complies with the general observation that "...warm or brackish water forms of a species of fish have.... fewer vertebrae than the forms inhabiting cooler or more saline water" (Hubbs, 1926).

Estuaries are well known for their high productivity, which is higher than that of many other maxine environments and makes them valuable as spawning grounds (Webb, 1973). Populations of F. nigripenne may be genetically adapted to this high productivity and as a consequence may produce smaller larvae with lower meristic counts. The cline from estuarine to strictly marine conditions would therefore be an expression of differences in temperature, salinity and productivity between these habitats. The presence of a cline also suggests that the estuarine form is not as yet reproductively isolated from the marine form, and hence separate specific status for each is not warranted (see review section).

The length at which skeletal elements begin to ossify initially is shown in Table I. Moser (1972) also includes the length of larvae and prejuveniles at which each element achieves its adult form. Selection of this length as Moser says is "...somewhat subjective since bone formation is a gradual process that continues throughout the life of the fish." The subjectivity is exaggerated in F. varium where the larval development extends over a greater size range $(5.85-27 \mathrm{~mm})$ than in the species studied by Moser (1972), Sebastes macdonaldi (Eigenmann and Beeson, 1893) which has a larval range from $4.5-1.5 .9 \mathrm{~mm}$. Because of the wide range in larval size in $\vec{F}$. varjum the size at which the adult form of each bone is attained is therefore not included here.

It is possible to make some generalizations about the sequence of ossification, which in very broad terms is similar to that in other teleosts (Goodrich, 1958; Mosex and Ahlstrom, 1970; Moser, 1972; Jollie, 1975). The skeletal elements begin to ossify over a wide range of larval lengths and the time it takes for each to acquire the adult characteristics also varies. The premaxillary forms at $8-9 \mathrm{~mm}$ and has most of the adult features by $19 \mathrm{~mm}$, a span of 10-11 mm. This can be compared with the ceratohyal which is laid down at $10.5 \mathrm{~mm}$ and becomes adult-like at $27 \mathrm{rm}$, thus spanning about $17 \mathrm{~mm}$. Functionally related elements tend to attain their adult form at about the same size. The maxillary arises before the premaxillary (7-8 $\mathrm{mm}$ and 8-9 $\mathrm{mm}$ respectively) yet they are equally ossified at $19 \mathrm{~mm}$. Similarly, bones covering the otic capsule and the roof of the neurocranium start to ossify at successive 
larval sizes but by $27 \mathrm{~mm}$ they are ossified to the same degree and meet at the margins. A similar trend is found in the opercular series. The sequences suggest that, as the fish grows larger and becomes more active, stress on the body increases, thus requiring the formation of certain "sets" of bones. The otic complex gives strength to the posterior chondrocranium, to which is attached the pectoral girdle and the anterior myomeres of the body, and these latter elements impose greater stress as swimming efficiency improves. The otic bones also indirectly support the jaws and opercular bones. Increased activity by these will require solid bracing (limited flexibility) in the otic region.

Large and strongly ossified bones in the adult seem to form first. For example the frontals, exoccipitals and the parasphenoid ossify earlier than the small otic bones such as the autosphenotic and the autopterotic. It is possible that earlier formation of the larger elements is necessary so that contiguous bones achieve a relative size, form and degree of ossification at the same time. Conversely the smaller and weaker the bone, the later it ossifies, as for example the ethmoid and sclerotics which are only faintly visible at $28.7 \mathrm{~mm}$. There appears to be no definite correlation between the size at initial ossification and the origin (endochonaxal or dermal) of a bone (Moser, 1972).

The onset of ossification of a bone is related somewhat to function. The caudal and jaw elements which presumably are necessary during the planktonic phase for swiming and feeding begin to ossify at a very small stage. The cleithrum also arises early, its appearance being due to its importance in anchoring muscles which help to open the mouth (Jollie, 1975). Similarly the early appearance of the opercular bones and the branchiostegals no doubt relates to their importance in respiration. Teeth (on jaws) and teeth plates (pharyngeal) are quick to develop, being required for initial predatory feeding. Teeth on the vomer and palatine do not appear till about $27 \mathrm{~mm}$ (comparatively late) in contrast to the early appearance of the same in Esox (Jollie, 1975). In Esox, however, vomerine and palatine teeth are the main toothed armament of the upper jaw, whereas in $\mathbf{F}$. varium they are of secondaxy importance and their late arrival may be a step toward their eventual loss.

The number of vertebrae are important diagnostic features of larval tripterygiids, clinids and gobiesocids, and therefore detection of these in young larvae is very important. Myomere counts which can be obtained in the small prolarvae correlate with vertebral counts but are more 
difficult to make. This difficulty is due to the poor definition of the myomeres in the anterior and caudal regions, accentuated by misleading folds of integument induced by formalin shrinkage of specimens. The bony centra develop first above the hindgut, then progress anteriorly and posteriorly from there. The anteriormost ones are completed very quickly so that the overall trend is for the centra to arise anteriorly and be progressively added to posteriorly. The length of the larval caudal region (posterior to the anus) in tripterygiids and clinids is large and hence the sequential development is exaggerated. The bony arches are also laid down in a similar manner. The centra of the vertebral column are ossified before all the arch bases and spines have formed (either in cartilage or bone). The earliest complete vertebral count is obtained from Alizarin stained specimens and not from those stained for cartilage by Victoria Blue or Toluidine. In F. varium a complete vertebral count can be made at about $15 \mathrm{~mm}$, but this differs in other species.

There is a direct relationship between the size of the adult and the larval size at which a structure first appears. As a genexal trend, the smaller the average size of the adult (tripterygiids, clinids and gobiesocids) the smaller the larval size at which the centra are ossified. The converse is also true, in that the larger the adult species the more "retarded" the development and hence the later (in terms of size) ossification takes place. This relationship also applies to other skeletal elements and to other diagnostic features such as pigmentation patterns etc. Knowledge of the relationship made possible in many cases the separation of the larvae of closely related species simply by size differences at particular stages of development, with the latter being determined by the degree of ossification of rays, spines, vertebrae, skull bones etc.

In $\mathrm{F}$. varium less than $15 \mathrm{~mm}$ long it is possible to distinguish "unossified" posterior centra by counting constrictions in the notochord. These constrictions are evidence of ossification although the material will not take up or retain the Alizarin stain. In Sebastes macdonaldi the neural arches are the first to ossify (anteriorly to posteriorly) followed by the centra in like manner (Moser, 1972). Each centrum forms from two ossification centres in $\mathrm{S}$. macdonaldi but this is not apparent in $\underline{\mathrm{F} \text {. varium }}$ where each centrum arises from one centre. 
The caudal rays may be counted earlier than any of the other rays, but because of the limited variation between the adults of different tripterygiid and clinid species the counts are of little diagnostic value. The urostyle in the caudal fin develops from a single centre of ossification giving no indication of possible origin from several centra as in the lower teleosts (Peckham, 1963).

The cartilagenous pterygiophores develop posteriorly first and then gradually anteriorly. Those of the rays are formed before those of the spines. This basic trend is modified by the tendency for the largest elements (or those supporting the largest) in a series to form before the rest e.g. the posterior pterygiophores of the anal fin appear before the anterior ones but the last rays but four are the longest in the adult and the pterygiophores of these develop before any which are posterior or anterior to them. The spines and rays of the dorsal and anal fins ossify long before their respective pterygiophore elements. Nevertheless the cartilagenous pterygiophores are present when the spines and xays ossify and are helpful (when stained) for reinforcing counts, especially in specimens with damaged fins or when spines and rays are tiny or poorly ossified. The cartilagenous pterygiophores can be stained rapidly and temporarily with methylene blue.* The posterior rays and spines are typically the first formed, although like the pterygiophores this sequence is modified by the tendency for the laxgest to arise first. The spine counts, especially those of the first dorsal fin, are very diagnostic for the tripterygiids and clinids. However, these spines are the last to ossify, and consequently are only important in diagnosis during late prejuvenile stages. The long fins exaggerate the sequential development of fin elements and hence give a "sliding scale" of development. Unfortunately much of this scale is subjective, and use of it depends on familiarity with the material being studied, overall fin shape, height of bony anlagen, and intensity of staining etc. objectively, however, ossified elements may be counted for different levels of development and compared with those of other species.

The pectoral rays ossify comparatively early (about $16 \mathrm{~mm}$ in F. varium) but are of little importance diagnostically as it is difficult to determine when the series is complete. Furthermore, in the tripterygiids the variation in adult pectoral ray counts is slight, although greater variation exists between clinids and tripterygiids and between different 
gobiesocid species. The completion of the complement of rays in the dorsal and anal fins is punctuated by the appearance of anterior spines; no spines are present in the pectoral fins. The completion of the second dorsal $f$ in is indicated by the first appearance of low spines in the anterior dorsal fin, which tend to arise simultaneously.

The overall. shape of the larva as determined by its skeletal configuration is diagnostic of genexa if not of species. Again this is difficult to assess objectively because of subtle variations in the angle of the preoperculum, the slope of the forehead (very shallow in Gilloblennius decemdigitatus (Clarke, 1879)), the shape of the snout and olfactory capsule, and the profile of the fins. Nevertheless these features are extremely important in an "intuitive" assessment of a larval series which may later be firmly defined by more standard methods. Osteological studies form an important basis for understanding overall larval and adult structure.

The presence of bone material is indicated by the uptake of Alizarin stain, and the amount of bone present is roughly proportional to the intensity of staining i.e. the darker the staining the more bone is present. The uptake of Alizarin is affected by the age of the specimen and the duration of washing prior to staining. Old and poorly washed specimens often stain poorly and give inaccurate pictures of overall ossification. Furthermore excessive destaining may cause loss of stain from delicate bony structures. It is important, therefore, to use relatively freshly fixed specimens and to standardize as near as possible staining procedures, especially if interspecific comparisons are to be made. 
TABLE I: Sequence of ossification of Forsterygion varium. The size at which skeletal elements ossify initially is shown.

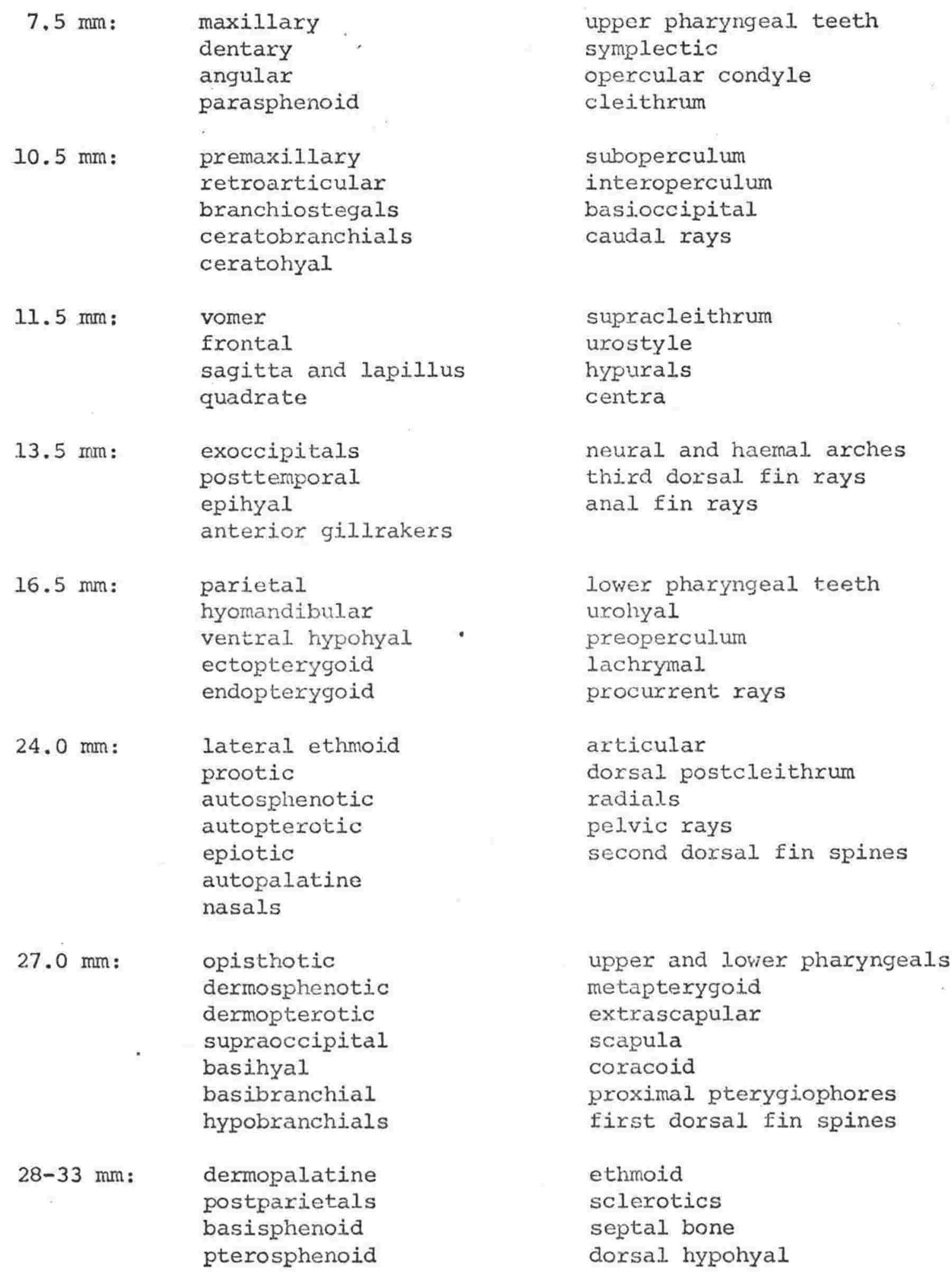


Abbreviations of the elements of the bony and cartilagenous

skeleton

Abbreviations: A, angular ("articular"); AC, anterior semicircular canal; AR, articular (endochondral ossification); AS, anal spine; BAL, otic bulla; BB, basibranchial; BH, basihyal; BO, basioccipital; BS, branchiostegal; $\mathrm{BSH}$, basisphenoid; $\mathrm{CA}$, foramen for carotid artery; $\mathrm{CB}$, ceratobranchial; $\mathrm{CH}$, ceratohyal; CL, cleithrum; CO, corocoid; CPRA, centrum of proatlas vertebra (basioccipital); CT, centrum; D, dentary; DR, dorsal ray; DS, dorsal spine; E, ethmoid; EB, epibranchial; ECT, ectopterygoid; EH, epihyal; ENT, endopterygoid (mesopterygoid); EO, exoccipital; EP, epural; EPB, epiphyseal bar; EPI, ethmoid plate; EPL, epural; EPO, epiotic; F, frontal; FM, foramen magnum; Fna, nasal fossa, FNCE, exit of facial nerve canal; HA, haemal arch; HB, hypobranchial; HC, horizontal semicircular canal; HM, hyomandibular; HS, haemal spine; HY, hypural; IH, interhyal; IOP, interoperculum; Ins, nasal septum; INT, integument; LA, lachrymal; LE, lateral ethmoid; LH, ventral hypohyal; LON, Lamina orbitonasalis; I.PH, lower pharyngeal (5th ceratobranchial); MC, meckels cartilage; MPT, metapterygoid; MX, maxillary; N, nasal; NA, neural arch; NS, neural spine; OC, olfactory capsule; OP, operculum; OPIS, opisthotic; OSP, septal bone; $P$, autopalatine; $\mathrm{PA}$, parietal; $\mathrm{PB}$, pelvic bone; $\mathrm{PBC}$, pelvic cartilage; PC, posterior semicircular canal; PCL, post cleithrum; PE, prethmoid; PER, pelvic ray; PM, premaxillary; POP, preoperculum; PP, medial extrascapular; PRtQ, palatopterygoquadrate cartilage; PR, pectoral ray; PRO, prootic; PS, parasphenoid; PSp, pelvic spine; PTO, autopterotic; PtP, pterygiophore; PTS, pterosphenoid; PTT, post-temporal; Q, quadrate; $R$, radial; $R A$, retroarticular ("angular"); S, schlerotic; SC, scapula; SCL, supracleithrum; SOC, supraoccipital; SOP, suboperculum; SPH, autosphenotic; ST, extrascapular; SY, symplectic; Tcom, trabecular communis; Tma, taenia tecti medialis anterior; TMS, taenia marginalis; TR, trabecula cranii; TSYN, tectum synoticum; Ttm, taenia tecti medialis; $U$, urostyle; UH, dorsal hypohyal; UPH, upper pharyngeal, URH, urohyal; vo, prevomer; $\mathrm{V}$, foramen for trigeminal nerve; VII, foramen for facial nerve; $\mathrm{X}$, foramen for vagus nerve. 
80.

Fig. 1. Helcogramma sp., $54 \mathrm{~mm}$ s.1. Lateral view of neurocranium suspensorium and opercular bones. 


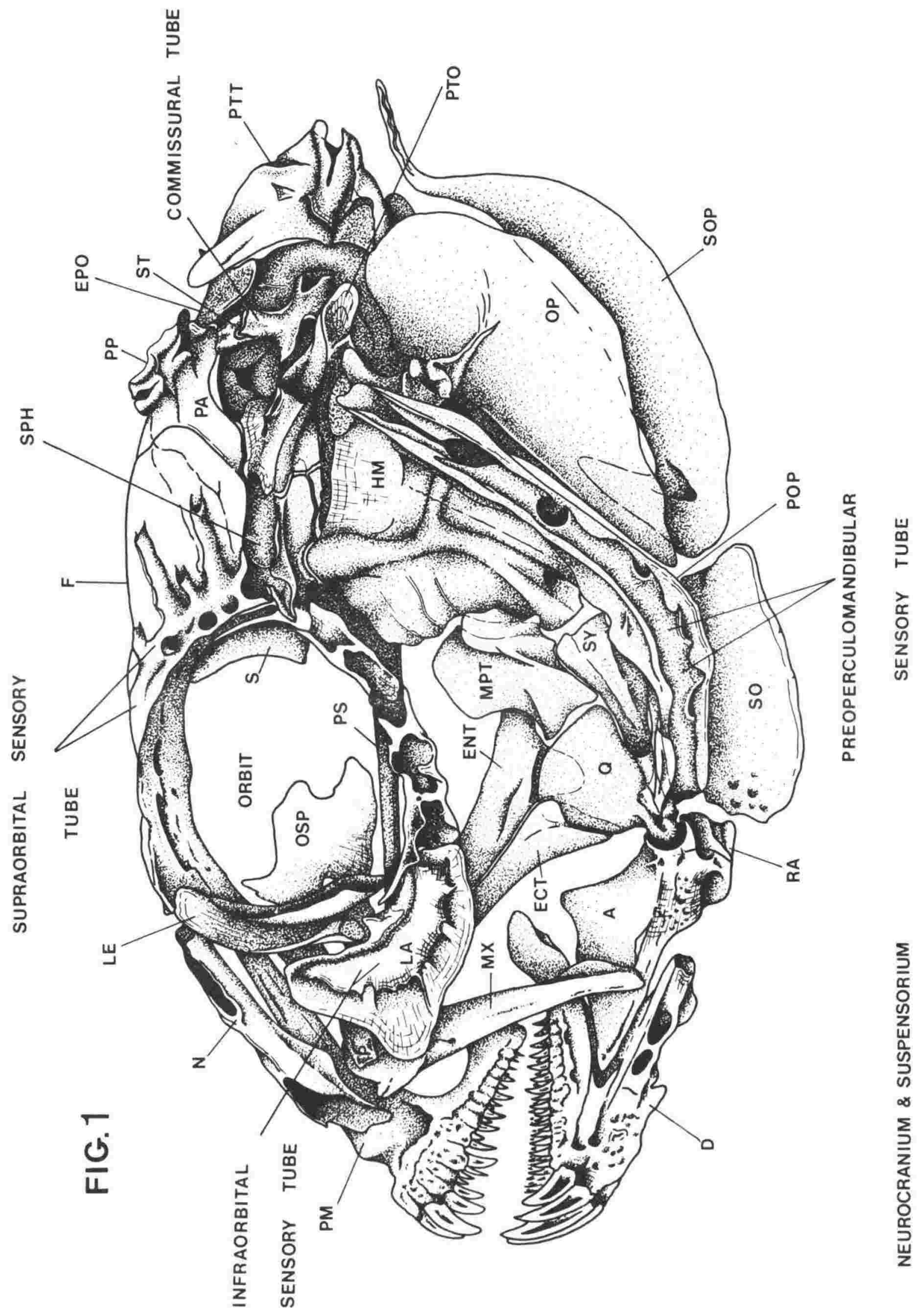


81.

Fig.2. Forsterygion varium $80 \mathrm{~mm} \mathrm{s.1.} \mathrm{No.1:} \mathrm{dorsal} \mathrm{view} \mathrm{of}$ neurocranium (postparietals missing); 2; ventral view of neurocranium. 


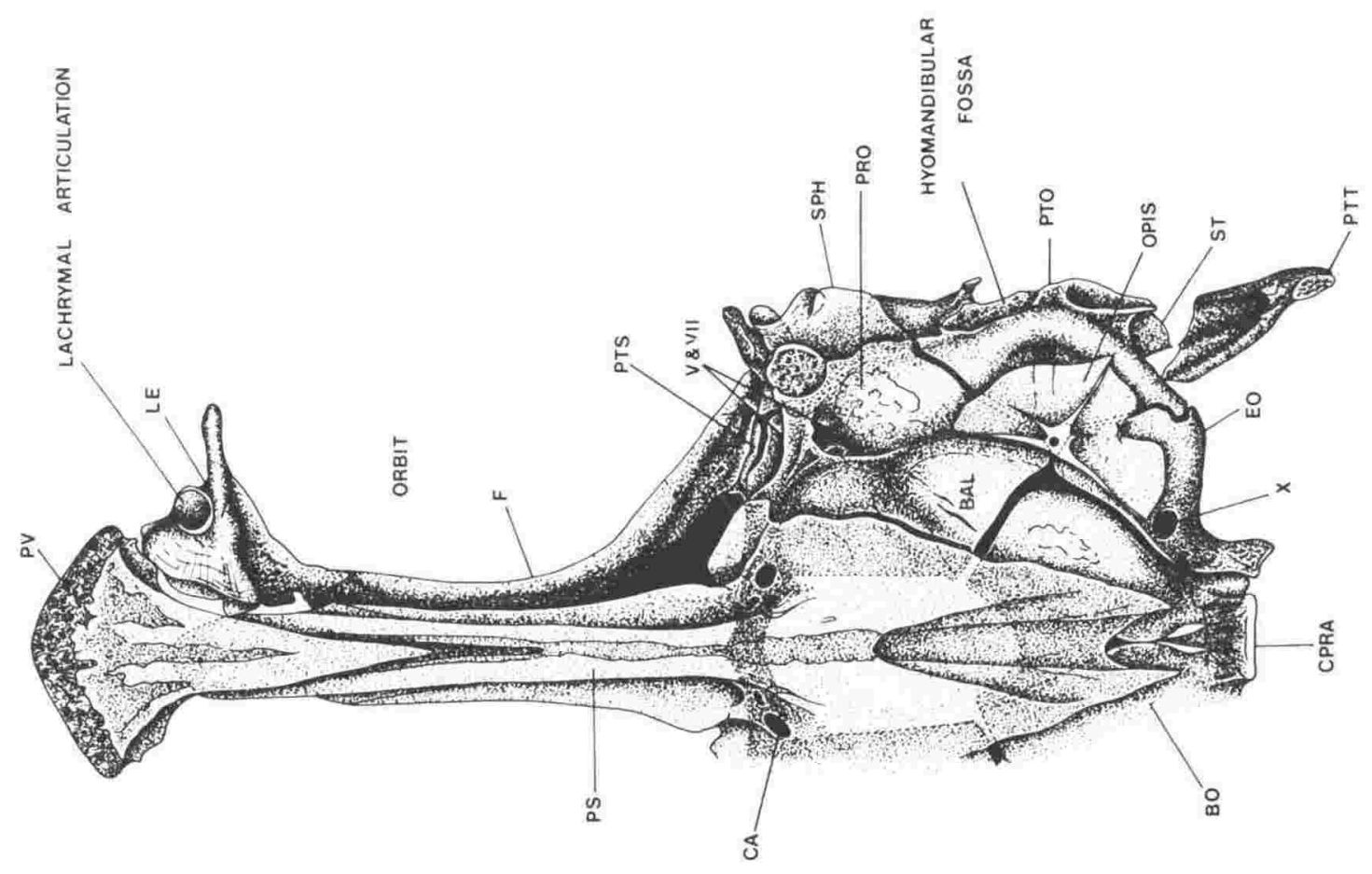

N

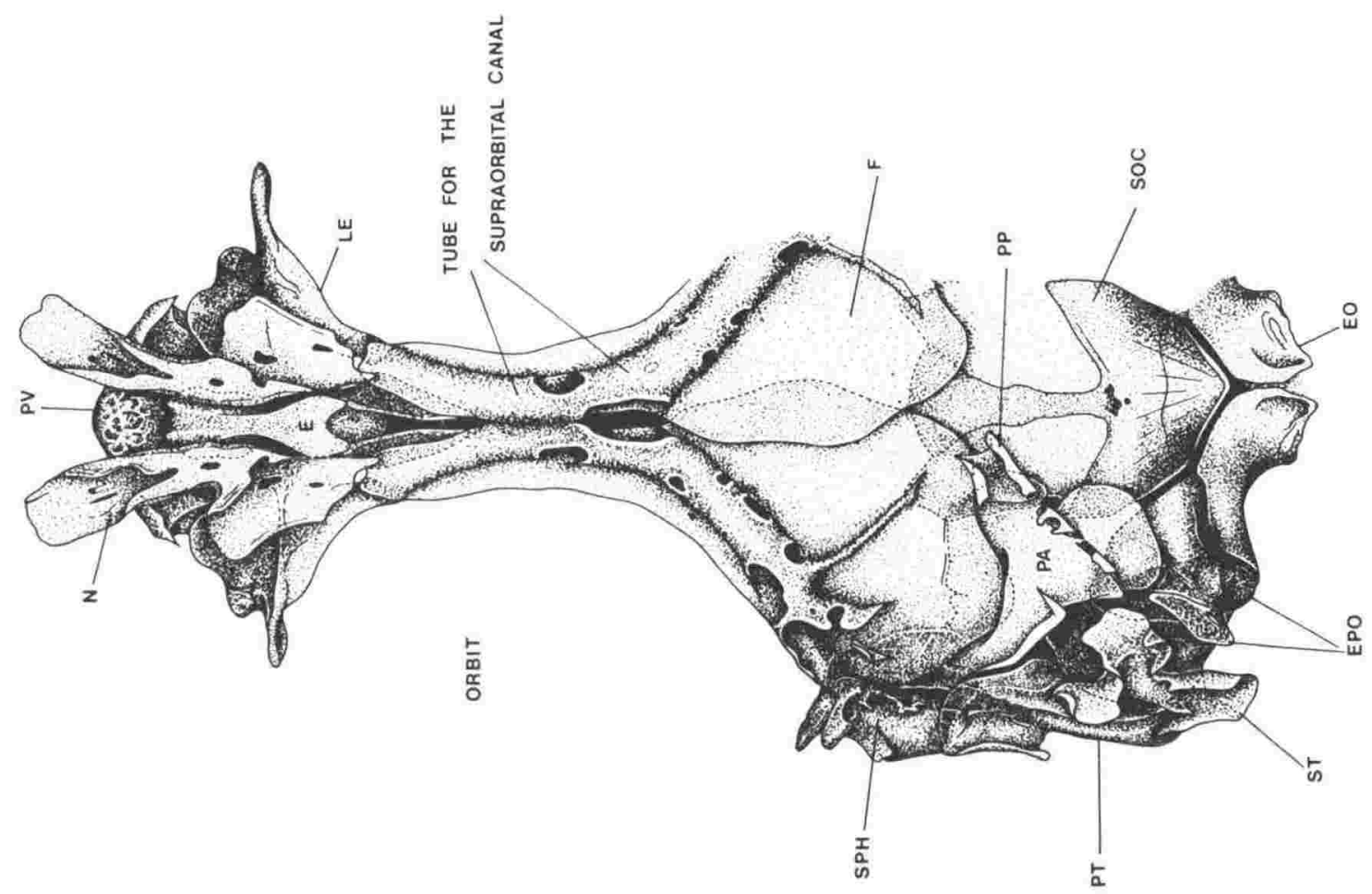

U.
$\frac{0}{4}$ 
82.

Fig. 3. Forsterygion varium $80 \mathrm{~mm}$ s.1. No.1: left lateral view of neurocranium; 2; posterior view of neurocranium, with opisthotic missing. 
FIG. 3

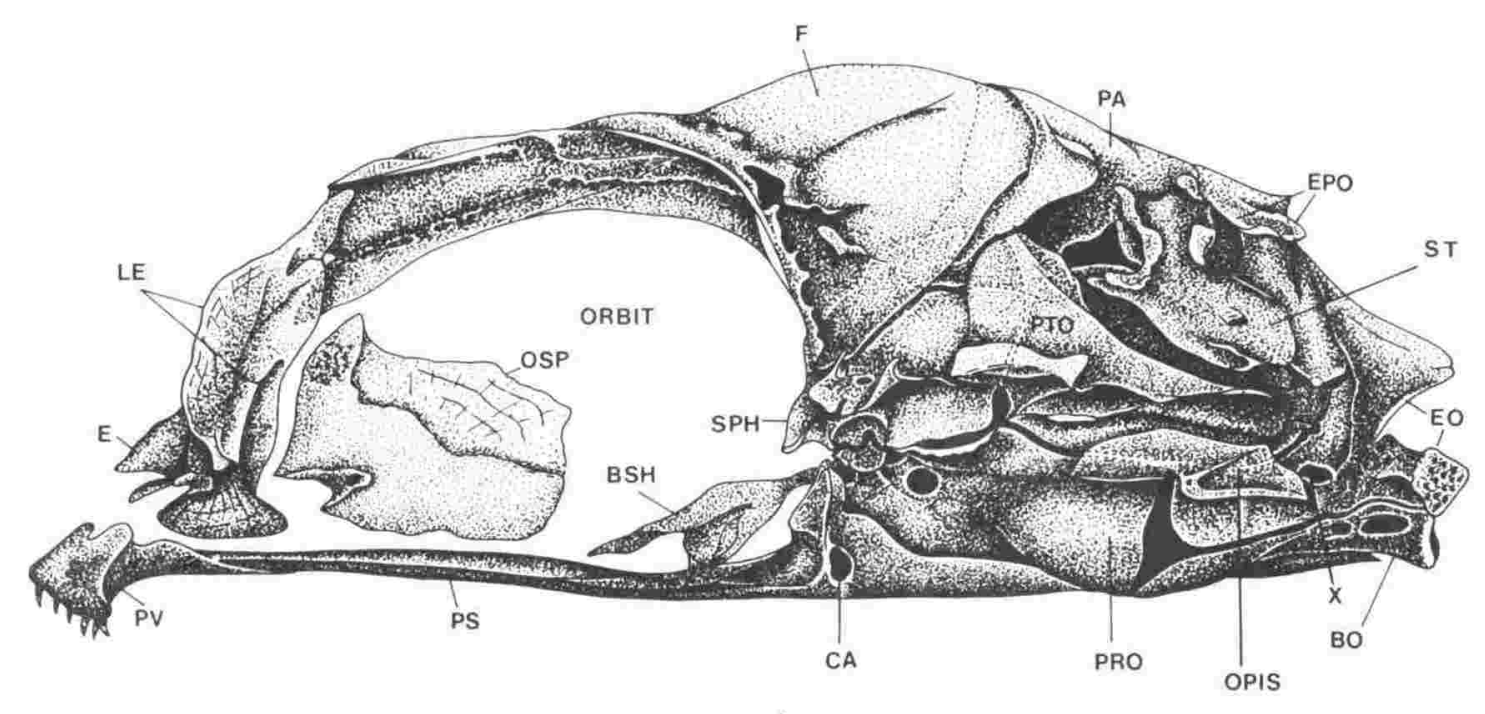

1

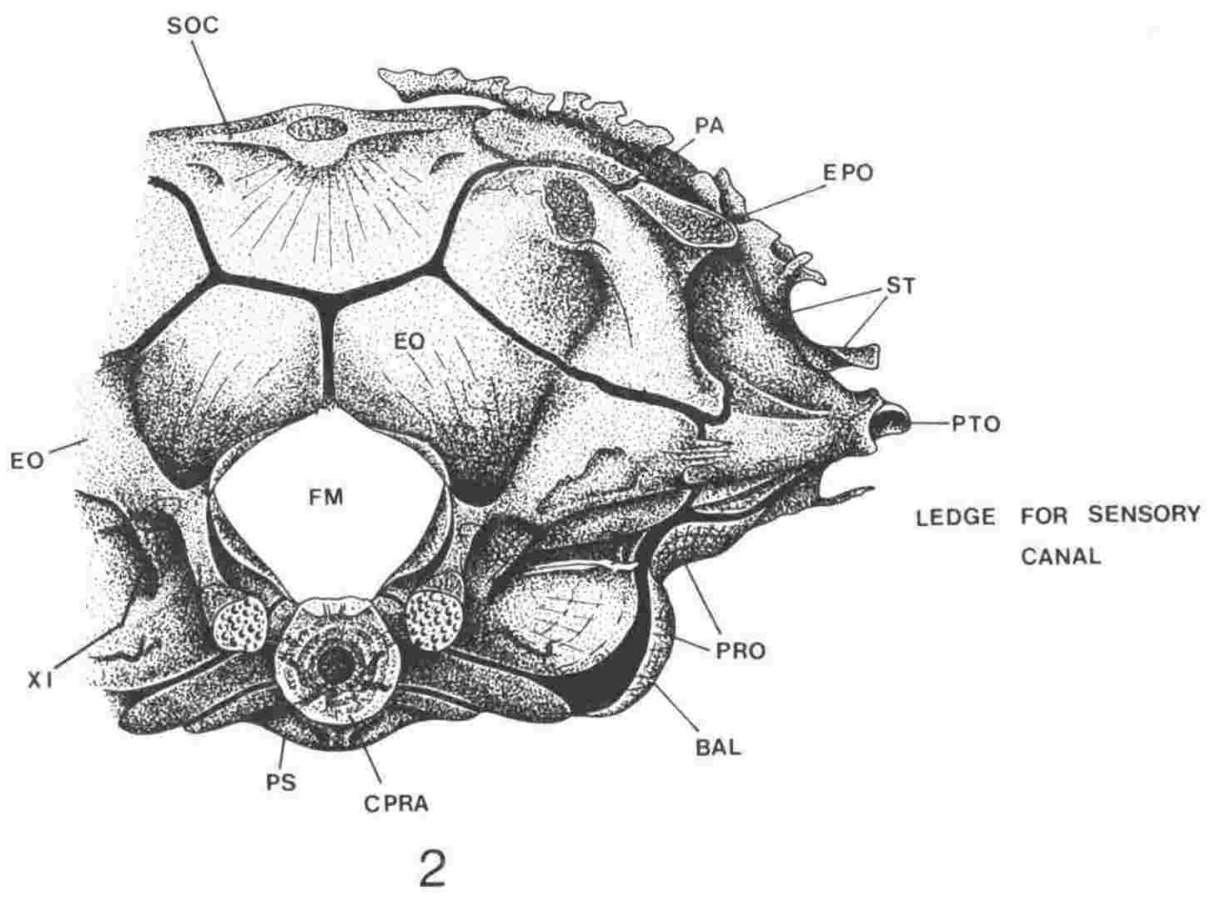


83.

Fig. 4. Forsterygion varium $80 \mathrm{~mm}$ s.1. No's 1 \& $1 \mathrm{a}$ : median and lateral view of left lower jaw; 2: left suspensorium and opercular bones; 3: premaxillary anterior view; 4: left circumorbital bones; 5: anterior view ethmoid bones and prevomer. 


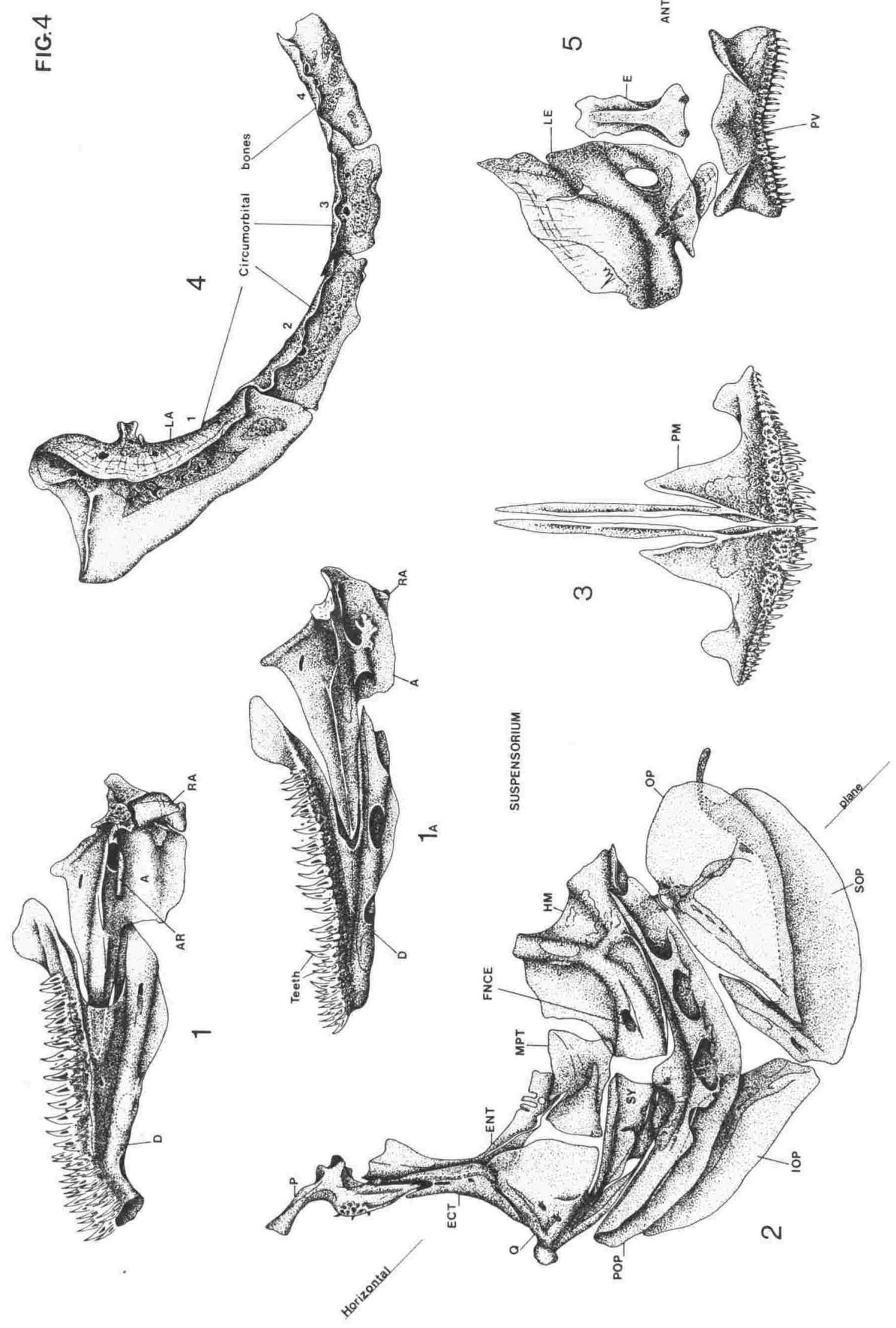


84.

Fig.5. Forsterygion varium $80 \mathrm{~mm}$ s.1. No.1: upper cleithral

elements of the left pectoral girdle; 2 \& $2 a$ : medial

and dorsal view of left pelvic girdle and rays; 3 \& $3 a$ :

lateral and medial view of left pectoral girdle;

4: left side of branchiocranium; 4a: 1st left ceratobranchial

with gill rakers and filaments; 5: left hyoid arch;

$5 a$; antero-yentrolateral view of urohyal. 


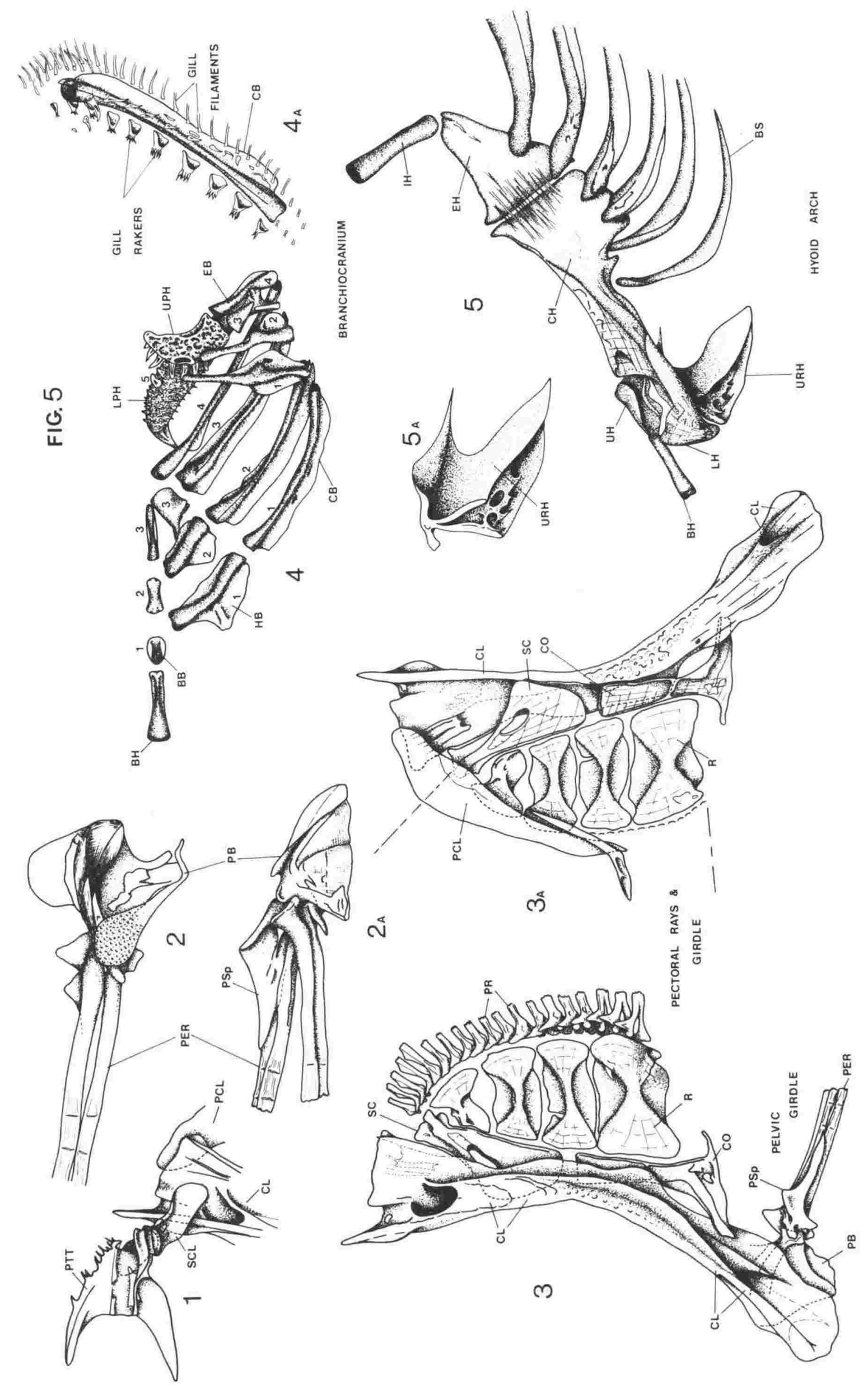


85.

Fig. 6. Forsterygion varium $80 \mathrm{~mm}$ s.1. No.1: 29th-33rd vertebrae with associated dorsal and anal fin skeletons; 2: caudal skeleton. 


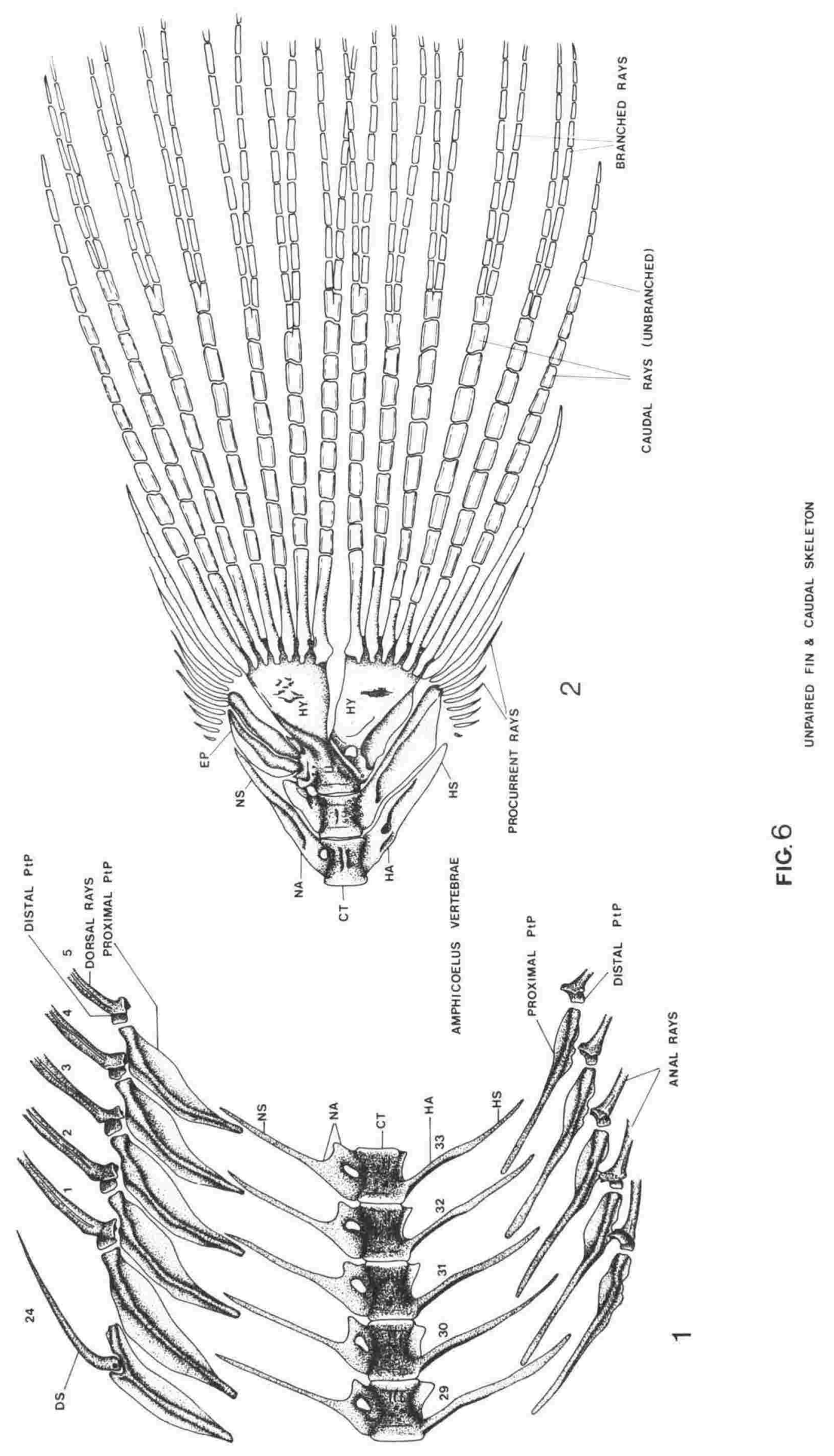


86.

Fig. 7: Forsterygion varium $80 \mathrm{~mm}$ s.1. No.1: anterior and abdominal vertebrae with associated dorsal fin skeleton; la: Ist vertebra; 2 ; 5 th vertebra; 3 ; 12 th vertebra; 4: 16th vertebra; 5: 29th vertebra, all vertebrae viewed anteriorly; 6; ventral view sagitta; 6a: lateral view sagitta; $6 \mathrm{~b}$ : ventral view asteriscus; $6 \mathrm{c}$ : ventral view lapillus. 


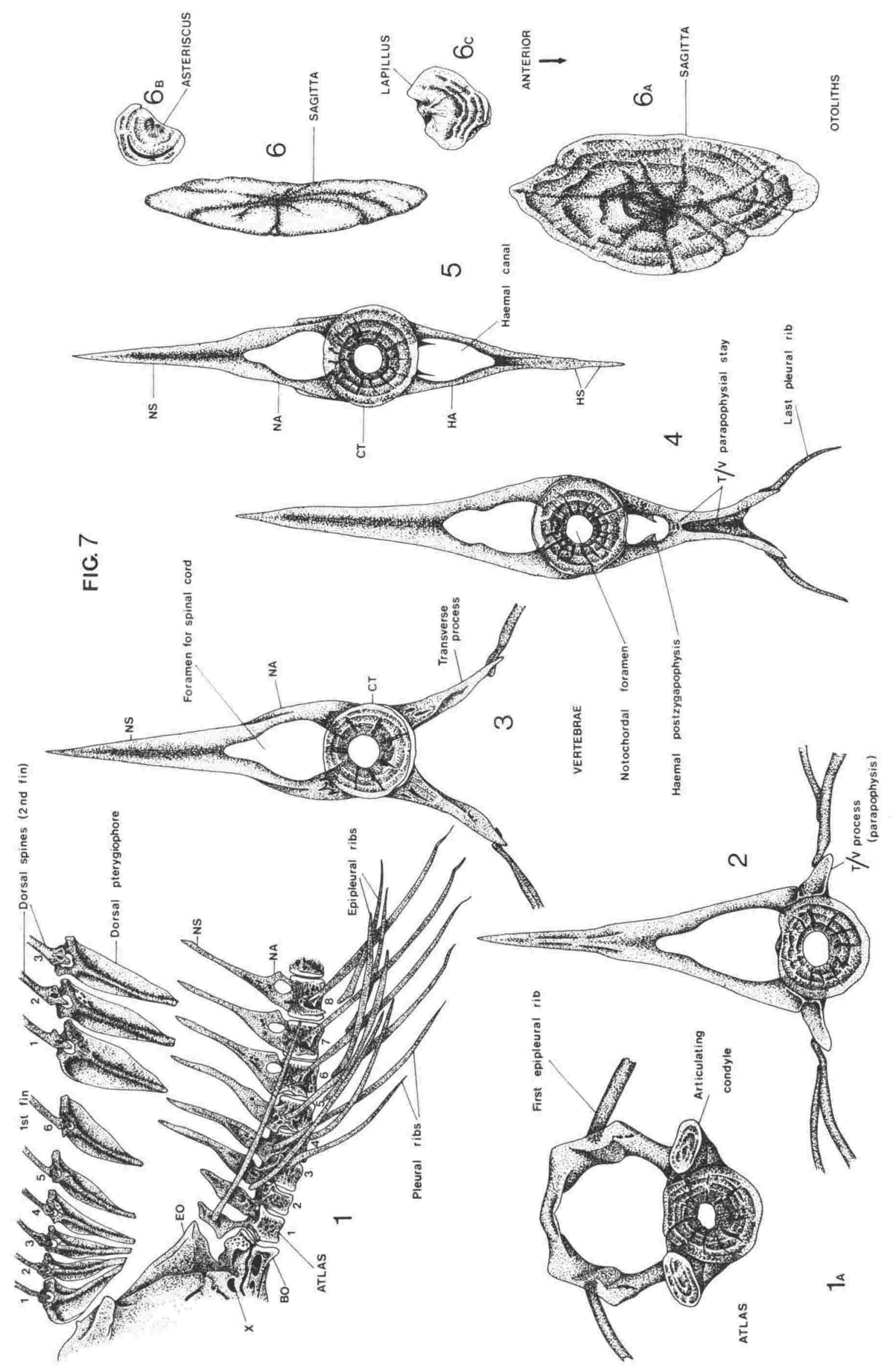


87.

Fig. 8. Forsterygion varium. No.1: $10.5 \mathrm{~mm}$ larva, lateral view of chondrocranium and suspensorium; 2: $10.5 \mathrm{~mm}$ larva, tail region showing caudal ray formation; $3: 16.5 \mathrm{~mm}$ larva, lateral view of chondrocranium and suspensorium; $4: 16.5 \mathrm{~mm}$ larva, 30th-32nd vertebral segments and associated rays. 


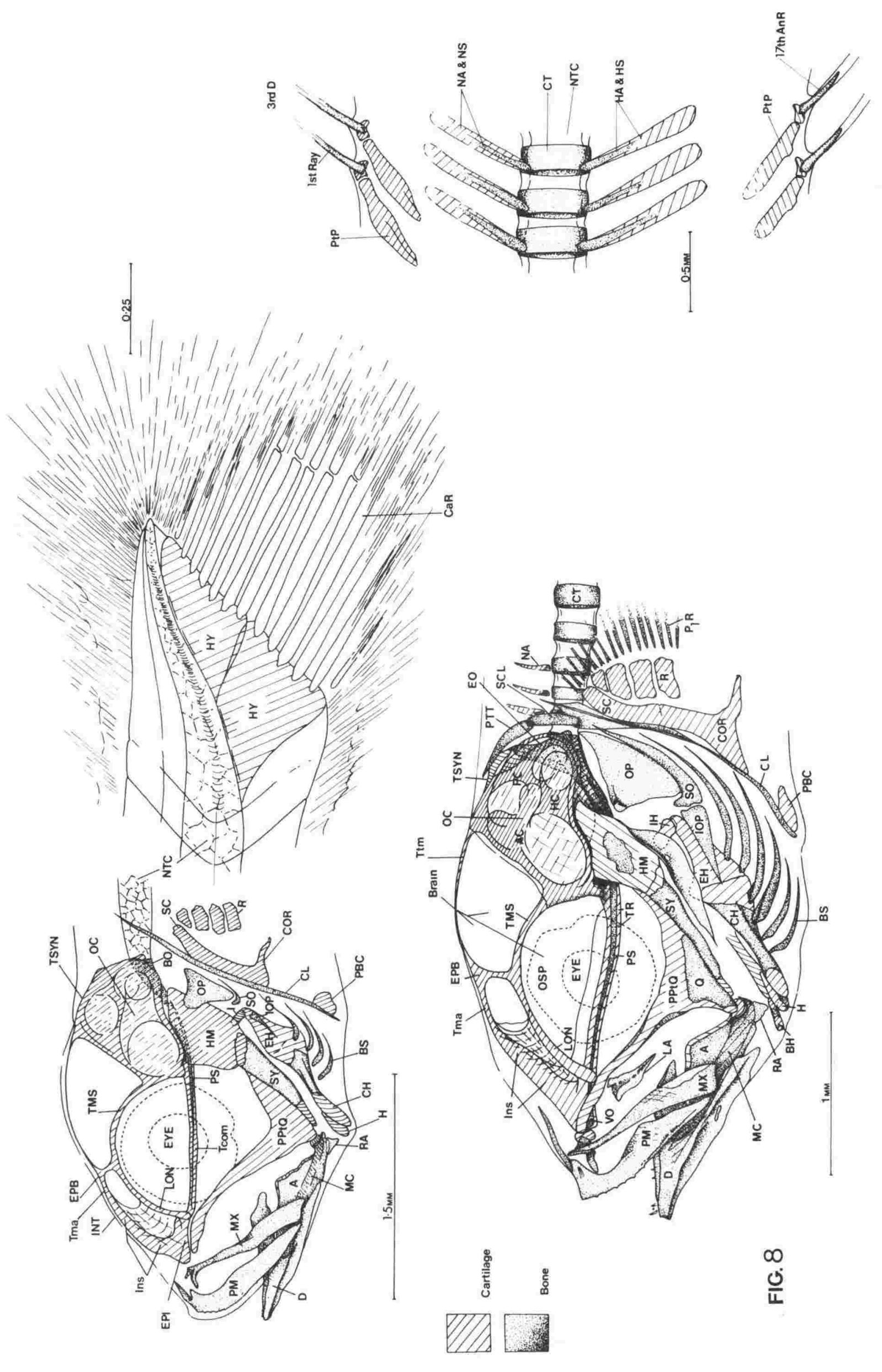


88.

Fig. 9. Forsterygion varium. No.1; $16.5 \mathrm{~mm}$ larva, dorsal view of chondrocranium and suspensorium; 2: $16.5 \mathrm{~mm}$ larva, posterior vertebrae, hypurals and caudal rays; $3: 27.0 \mathrm{~mm}$ prejuvenile, ventral view of chondrocranium showing areas of ossification; 4: $27.0 \mathrm{~mm}$ prejuvenile, dorsal view of neurocranium and suspensorium (cartilage not shown). 


89.

Fig.10. Forsterygion varium. No.1: $27.0 \mathrm{~mm}$ prejuvenile, lateral view of neurocranium and suspensorium; 2: $27.0 \mathrm{~mm}$ prejuvenile branchiocranium; $3 \& 4: 27.0 \mathrm{~mm}$ prejuvenile, vertebrae; $5,6 \& 7 ; 27.0 \mathrm{~mm}$ prejuvenile, spines and rays of dorsal and anal fins. 

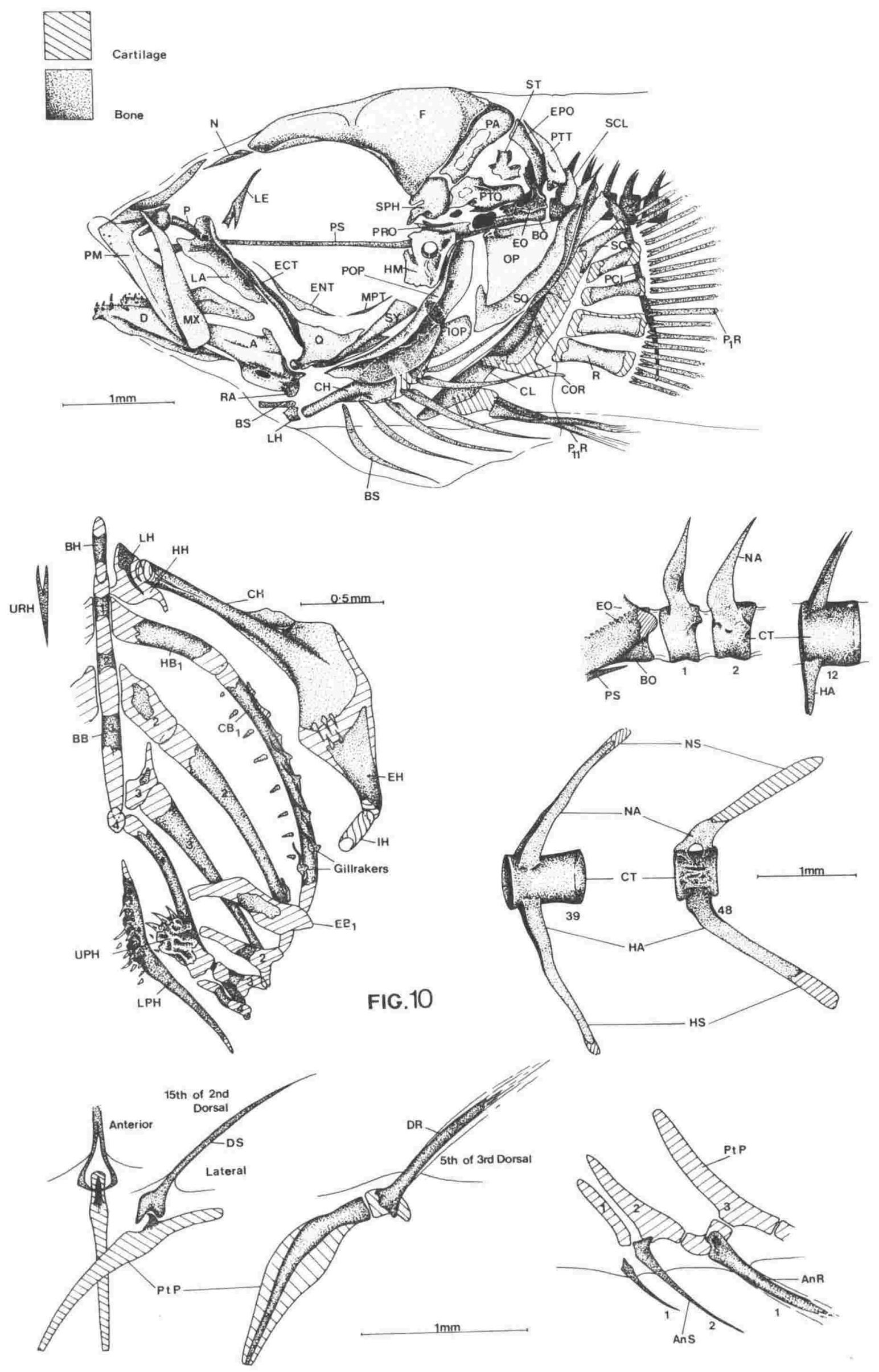
90.

Fig.11. Forsterygion varium. No.1: $23.0 \mathrm{~mm}$ prejuvenile, lateral view of anterior chondrocranium; 2: $23.0 \mathrm{~mm}$ prejuvenile, dorsal view of anterior chondrocranium. 


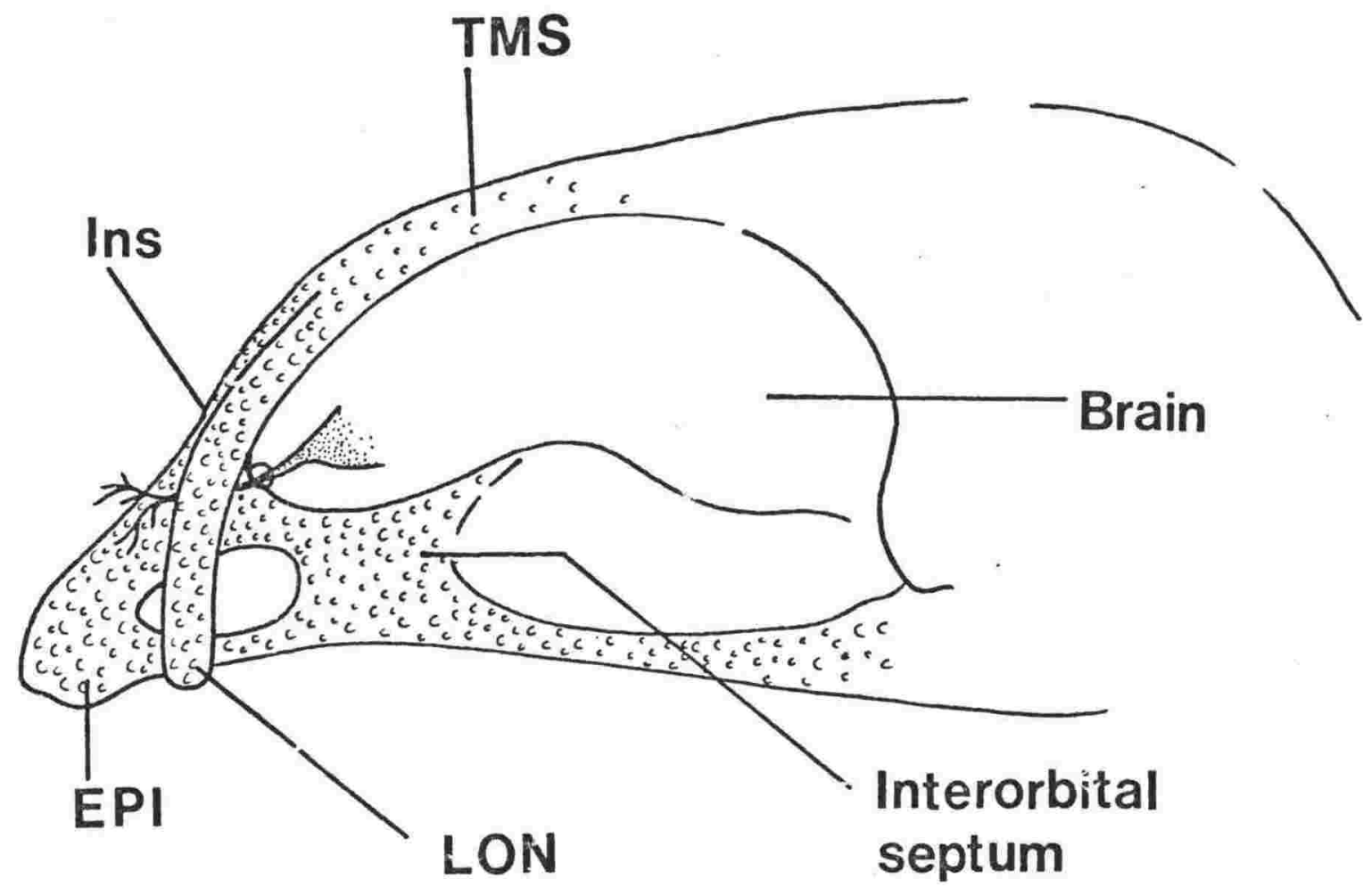

1

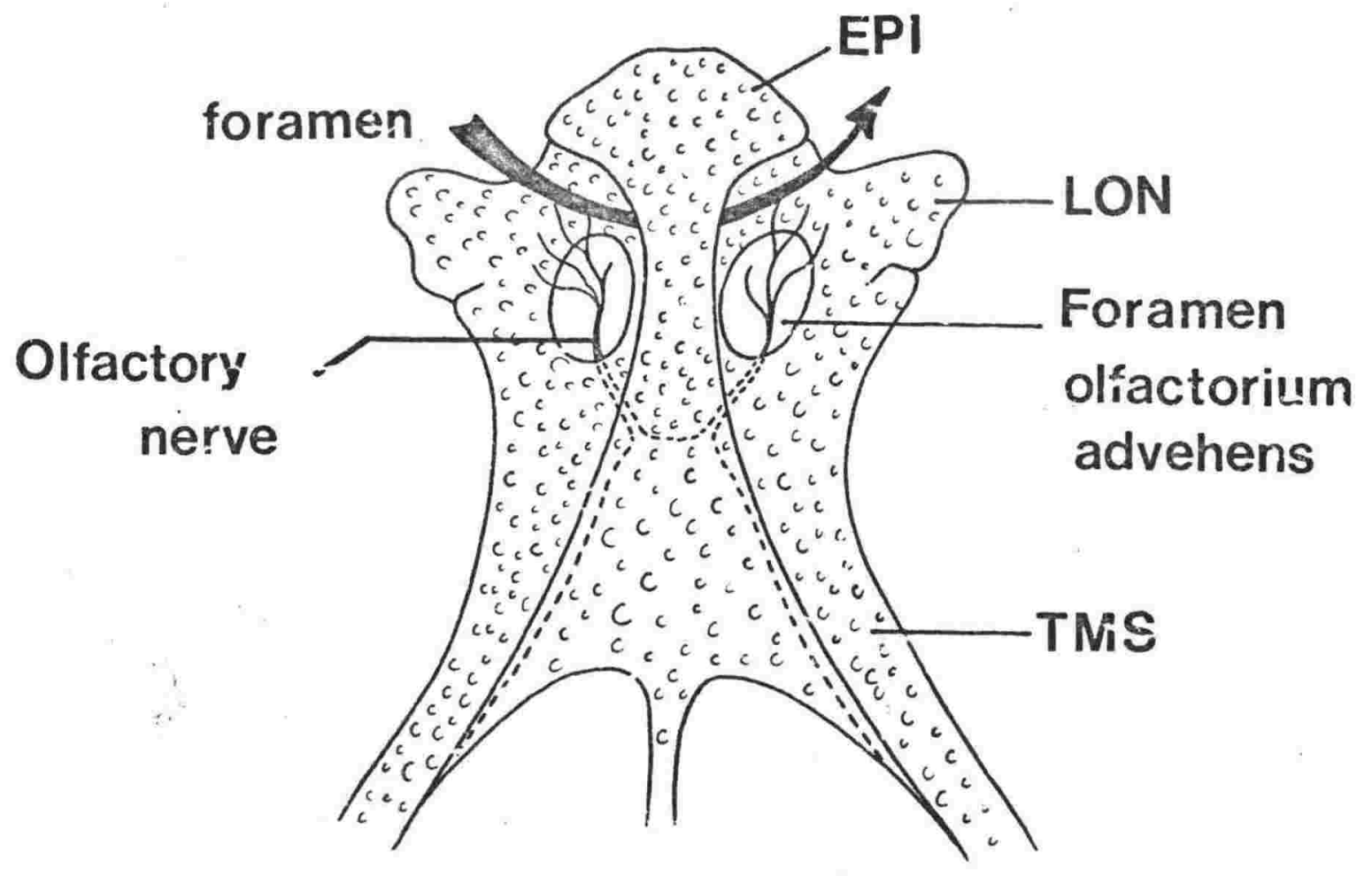

2

Fig 11 no's 1 and 2 
Development of the Lumpfish, Trachelochismus melobesia

(Pisces: Gobiesocidae)

By J. G. Ruck,

Department of Zoology, Victoria University of Wellington. Zoology Publications fxom Victoria University of Wellington, No.57, issued, August, 1971.

\section{Abstract}

In the Wellington area eggs of T. melobesia Phillipps, 1927 are laid during spring in clusters of three eggs to several hundred on the under-surface of permanently tide-covered stones. The eggs are oval with a flattened, adhesive base, many oil globules and average $1.65 \mathrm{~mm} \times 1.35 \mathrm{~mm}$. Egg clusters are attended by one parent until hatching. Under laboratory conditions this occurs at 12 days in temperatures of about $15^{\circ} \mathrm{C}$. The emergent prolarva is $4.8 \mathrm{~mm}-5.5 \mathrm{~mm}$ standard length and has a prominent yolk sac with a single large oil globule. There are scattered melanophores above the gut and a conspicuous yellow tinge surrounds the brain, extending in the lateral miscle mass to the 8 th post-anal somite. Larvae of $6.5 \mathrm{~mm}-7.85 \mathrm{~mm}$ standard length, and more advanced in development, occur in the Island Bay plankton from early September to late December.

\section{Introduction}

Trachelochisnus melobesia Phillipps, 1927, is an endemic lumpfish (Family Gobiesocidae) not uncommon on the shore in semi-exposed rock and rubble areas in the Cook strait region of New zealand. It is found throughout the inter-tidal zone but appears to be most abundant near the

N.B. This article has been published. In this thesis, however, photographs of the embryological development replace sketches found in the original. The larval stages are described briefly but the drawings are excluded, as they are more adequately portrayed in a later section. 
upper low-tide level. Adults rarely attain lengths of more than $30 \mathrm{~mm} \mathrm{s.1}$. Trachelochismus pinnulatus (Forster in Bloch and Schneider) is also found in areas inhabited by T. melobesia. However, the adult of T. pinnulatus (maximum size $71.2 \mathrm{~mm}$ s.1.) is larger than that of $\mathrm{T}$. melobesia, and lacks the reddish-purple patch on the dorsal surface, a character which readily identifies the latter species. Flattened papillae occur on the central region of the sucker disc of $\mathrm{T}$. melobesia, but are absent in $\mathrm{T}$. pinnulatus. The two species are further distinguished by fin ray counts (Briggs, 1955: 19-20) as follows:-

$$
\begin{aligned}
& \text { T. melobesia D10 }(9-11), \text { A8 }(7-8), \text { P1 } 23(22-24), \text { C12 } \\
& \text { T. pinnulatus D8 }(7-9), \text { A6 }(5-7), \text { P1 } 25(24-26), \text { C12 (11-12) }
\end{aligned}
$$

The present study describes the egg and larval development of T. melobesia. The life history of T. pinnulatus has been studied by Coakley (1964), and Graham (1939, 1953) has briefly described the egg and prolarval stages of Diplocrepis puniceus (Richardson, 1846), a further gobiesocid known fxom the New Zealand seashore. The family is otherwise poorly known in respect of early life history. Howevex, comprehensive accounts of the life history of the South American clingfish Gobiesox strumosus Cope, 1870 are gjven in Runyan (1961: 113-141) and Dovel (1963: $161-166)$.

\section{Materials and Methods}

On 20.10.69 four egg masses on stones were collected from the western shore of Lyall Bay and kept in rectangular plastic containers $(30 \times 28 \times 13 \mathrm{~cm})$ at the Island Bay Marine Laboratory. The watex was changed daily and kept constantly aerated using an eddy-current aix pump. The stones with eggs were wedged at a $45^{\circ}$ angle against the sides of the container's with the egg masses on the ventral surface. Porous "air stones" were placed beneath the eggs so that the streams of small bubbles issuing from the "air stones" flowed over the egg masses. This method prevented the accumulation of detritus on the eggs and ensured an adequate supply of oxygenated water. Despite these precautions many eggs died and these were removed each day to prevent the build up of fungi and bacteria. The eggs adhered very closely to the stones and those required for observation were very difficult to remove without damage to the egg membrane. Iimited 
success was obtained by sliding a sharp scalpel between the egg and the stone and then pipetting the dislodged egg into a petri dish. The identity of the above eggs as those of T. melobesia was confirmed by comparing these with thirty eggs laid on 30.10 .69 by one of three females (22, 23, 26 mm s.1.), which together with two males $(27,29 \mathrm{~mm} \mathrm{s.1.)}$ were kept alive in an aquarium. Development of these eggs and those obtained earlier was studied and photographed using a Timex camera. A micrometer ocular was used for measuring eggs and larvae. Measurements of the larvae were based on those recommended in Hubbs and Lagler (1958: 24-26).

Larvae larger than $5.3 \mathrm{~mm}$ were obtained during the spring by making regular plankton tows approximately 100 metres offshore at Island Bay from a small boat. The net used was of standard conical design with a $57 \mathrm{~cm}$ diameter opening and a mesh size of 500 microns.

\section{Spawning and Development}

In the Lyall Bay and Island Bay areas near Wellington T. melobesia spawns from early september to late December. Egg masses are found beneath smooth stones which usually remain covered with water at low-tide. The egg clusters range from $0.5 \mathrm{~cm}$ in diameter, containing as few as three eggs, to about $8 \mathrm{~cm}$ in diameter and containing several hundred eggs. The eggs are laid close together forming flat, irregular-shaped masses.

The larger egg clusters always contain groups of eggs which differ in colour, ranging from bright crimson to pale pink. This variation in colour represents the progressive depletion of the yolk supply in the eggs of each group as development advances. It is strongly suggested therefore that the eggs are laid at different times on the same stone. The pale yolked eggs, being the most advanced in development, are found mainly in the middle of the egg masses. However, the difference in development of eggs of some adjacent groups is relatively very small, indicating that these egg groups are laid no more than one to two days apart.

The absolute fecundity of T. melobesia is apparently very low, as determined from 10 gravid females each of which was found to contain only 20-30 xipe eggs. Considering the apparent low fecundity and the close development of the adjacent groups within a cluster it is concluded 
that more than one female is responsible for egg clusters that contain several. hundred eggs.

Approximately 60 egg clusters wexe examined in rock pools in the Wellington area, of which about $80 \%$ had an adult T. melobesia in attendance. In these cases it was not cleax whether this was a male or an immature female. When two adults were found close to the eggs the female could be distinguished by her characteristic pink and distended belly (the condition of a gravid female). Parental vigilance occurs commonly in littoral fish. Gibson (1969: 385) observes that predation on the eggs is prevented by the guardian activities of one of the parents, usually the male. Runyan (1961: 118) also states that "...eggs in good condition were usually accompanied by a rnale Gobiesox strumosus, that kept them constantly aerated by fanning anal and caudal fins. .."

\section{Development of the Egg}

The mean dimensions of 100 eggs were $1.65 \times 1.35 \mathrm{~mm}$. The eggs are oval and dorso-ventrally depressed and are attached to the substrate by a flattened adhesive base. When first laid the eggs are bright crimson and are about $1.2 \mathrm{~mm}$ long, but within minutes they expand to a length of $1.65 \mathrm{rm}$, by uptake of watex through the egg membrane (Fig.1, no.1). The yolk is central. and subspherical and has a mean diameter of $1.3 \mathrm{~mm}$. The yolk of the fertilised egg is a crimson-red colour, and contains between 10 and 100 oil globules, one of which becomes doninant during latex development. Development to hatching (Figs 1-2, no's 1-12) took 12 days at a water temperature of $15^{\circ} \mathrm{C}$.

Two hours (Fig.1, no.2). A single large cell appears from beneath the yolk and later expands and moves to one end of the yolk within one of the lateral perivi.telline cavities.

Three hours (Fig.l, no.3). The first cleavage divides the cell dorso-ventrally relative to the egg base, and at right angles to the yolk surface. The cells formed round off and appear as two swellings protruding from one end of the yolk-sac.

Four hours (Fig.1, no.4). The four celled stage is produced by a second cleavage at right angles to the first. Oil globules are 
free to move and tend to migrate to the uppermost region of the yolk; hence their position depends on the attitude of the egg.

Five hours. In the eight celled stage the blastomeres are still in a single layer. The cells are arranged in two rows of four cells each.

Six hours. The sixteen celled stage is reached with the cells beginning to form a round blastodisc.

Twenty hours (Fig.1, no.5). The blastula is well formed and consists of a prominent cap of cells, (blastodexm) beneath which lies the blastocoel.

Forty hours (Fig.1, no.6). At this stage the blastodisc has spread halfway around the yolk, in so doing obliterating the blastocoel. Epiboly is not obvious, except for the slight thickening of the lateral. rim of the blastodisc.

Sixty-six hours (Fig.1, no.7). The blastodisc covers the entire yolk-sac. The embryological axis is well defined and lies deeply notched into the yolk, particularly in the cephalic region.

Eighty-five hours (Fig.1, no.8). The outline of the embryo is distinct, encircling half the yolk-sac. The myotome rudiments and the auditory placodes are just visible. The main oil globule has become larger, apparently at the expense of the smaller oil droplets which have slowly decreased in size. Fore-brain development is quite evident, and the lens are beginning to thicken.

Fourth day. At this time the embryo encircles more than half of the yolk and the tail begins to extend free fxom the yolk-sac. The oil globule lies directly beneath the tail and remains here for the rest of the development. Optic cups contain outlines of the lenses, and there is a pronounced enlargement of the hindbrain. Approximately 17 myomeres are presént.

Fifth day (Fig.2, no.9). The total body length has increased slightly. Nearly all the myomeres are present posterior to the otic vesicles and extending well down into the tail. At this time the heart begins to beat very faintly, but blood movement is seen only in the region of the heart. The heart lies well forward beneath the head and is obscured by the yolk. 
Seventh day (Fig.2, no.10). The yolk is reduced considerably and the ventral aspect of the embryo faces upwards. The tail is well formed and is turned back to lie parallel with the body. The gut is formed and has scattered pigment spots on its upper surface. The heart beats regularly and strongly. Each beat of the heart sends a wave of movement through the yolk. Blood flows in the dorsal and ventral blood vessels and the vitelline vessels are large and run laterally across the yolk. Pigment has appeared in the chorioid of the eye.

Ninth day (Fig.2, no.11). Little change has taken place except for an increase in pigmentation of the eyes and the peritoneum.

Twelfth day (Fig.2, no.12). The mouth and external nares are formed and the gut pigmentation has become darker. There is a further reduction in the amount of yolk. Pigmentation of the chorioid appears complete. The embryo is cramped within the egg and the tail arches forward to overlap the head. Just prior to hatching the embryo becomes agitated and begins to flex its tail. As a result of this activity the chorion is ruptured and the prolarva is released.

Prolarvae. Some of the larvae that hatched in the laboratory may have been induced to do so prematurely, as a result of disturbance. This is suggested by the variation in the amount of yolk present in each prolarva immediately after hatching. Prolarval length on hatching ranges from 4.8-5.5 m. At this stage the gut is long and extends to the base of the 15th myomere. The upper peritoneum of the gut is covered with numerous stellate melanophores, extending from above the yolk to the vent. Posterior to the gut are 1.6-18 myomeres. Stellate melanophores are present in the myomeres just past the vent, but their number is variable. An obvious yellow tinge surrounds the braincase, and this extends through the myotomes above the gut to the $8 \mathrm{th}$ myomere past the vent. All larvae kept in the laboratory died within 3 days of hatching.

Larvae. At $6.5 \mathrm{~mm}$ the yolk-sac is almost absorbed and the gut reaches beyond the mid-length of the body. The oil globule is no longer visible. Pigmentation has changed very little except for an increase in the size of the melanophore at the base of the pectoral fin. The jaws are well formed and appear functional, although as yet are without well defined teeth. Two gills and 6-7 branchiostegal rays are visible on either side of the head. Two sucker buds lie ventral to the gills and heart. The longitudinal fin folds have slightly increased in size. 
At $7.85 \mathrm{~mm}$, the overall shape of the larva changes slightly. Essentially there is a flattening and broadening of the head, and an increase in the depth of the tail. In addition to the existing pigment pattern, numerous grey spots are scattexed about the outer edges of the larvae. The dorsal, anal and caudal fins show signs of ray formation. The urostyle is curved upwards and extends a considerable distance into the caudal fin. The ventral sucker is well developed and functional. At this small size the larvae are able to cling to the sides of glass jars. No specimens larger than $7.85 \mathrm{~mm}$ were caught in the plankton nets. Because larvae of this size have a fully functional sucker it is suggested that at this stage they attach beneath stones.

The average measurements of 25 prolarvae are as follows:

\begin{tabular}{|c|c|c|}
\hline Standard length (mm) & & 5.40 \\
\hline Total length (mm) & & 5.70 \\
\hline Head length (mm) & & 0.95 \\
\hline Eye length (mm) & & 0.41 \\
\hline Snout to anus (mm) & & 3.00 \\
\hline Larval measurements are as & Lows: & \\
\hline Standaxd length (min) & 6.50 & 7.85 \\
\hline Predorsal length (mm) & 3.20 & 5.00 \\
\hline Head length (mm) & 1.40 & 2.15 \\
\hline Depth of head (mm) & 0.90 & 1.21 \\
\hline Snout length (mm) & 0.31. & 0.50 \\
\hline Eye length (mm) & 0.50 & 0.57 \\
\hline Interorbital distance ( $\mathrm{mm}$ ) & 0.35 & 0.55 \\
\hline Head width (mm) & 1.10 & 1.40 \\
\hline
\end{tabular}

Acknowledgement

I would like to thank Dr P.H.J. Castle, Department of Zoology, Victoria University of Wellington, for his constructive criticism of this paper. 


\section{Literature Cited}

Briggs, J. C. 1955.

A monograph of the clingfishes (Order Xenopterygii). Stanford Ichthyol. Bull., 6, 224 pp., 114 fig., 1 tab.

Coakley, A. 1964.

Life history and general biology of Trachelochismus pinnulatus

(Forster) (Order Xenopterygii). Unpublished M.Sc. thesis,

University of Canterbury (not seen).

Dovel, W. L. 1963.

Larval development of clingfish, Gobiesox strumosus, 4.0 to 12.0

millimeters total length. Chesapeake Sci., 4(4): 161-166, 6 fig.,

$3 \mathrm{tab}$.

Gibson, R. N. 1969.

The biology and behaviour of littoral fish. Oceanogr. Mar. Biol.

Ann. Rev., 7: 367-410, 5 fig., 3 tab.

Graham, D. H. 1939.

Breeding habits of the fishes of Otago Harbour and adjacent seas.

Trans. Proc. R. Soc. N.Z., .69: 361-372, 7 pl.

Graham, D. H. 1953. Treasury of New Zealand fishes. (Wellington:

A. H. \& A. W. Reed). 404 pp., 153 fig.

Hubbs, C. L. \& Lagler, K. F. 1958. Fishes of the great lakes region.

(Michigan: Cranbrook Institute of Science). 213 pp., 251 fig.,

6 tab.

Runyan, S. 1961.

Early development of the clingfish, Gobiesox strumosus Cope.

Chesapeake Sci., $2(3,4)$ : 113-141, 33 fig., 6 tab.

J. G. Ruck, B.Sc. (Hons),

Department of zoology,

Victoria University of Wellington,

P. O. Box 196,

Wellington, New Zealand. 
99.

Fig. 1. Trachelochismus melobesia. No.1: Fertilized egg just following the uptake of water; 2 : two hours; $3: 3$ hours; 4: 4 hours; $5 ; 20$ hours; $6: 40$ hours; $7: 66$ hours; 8: 85 hours. 



5
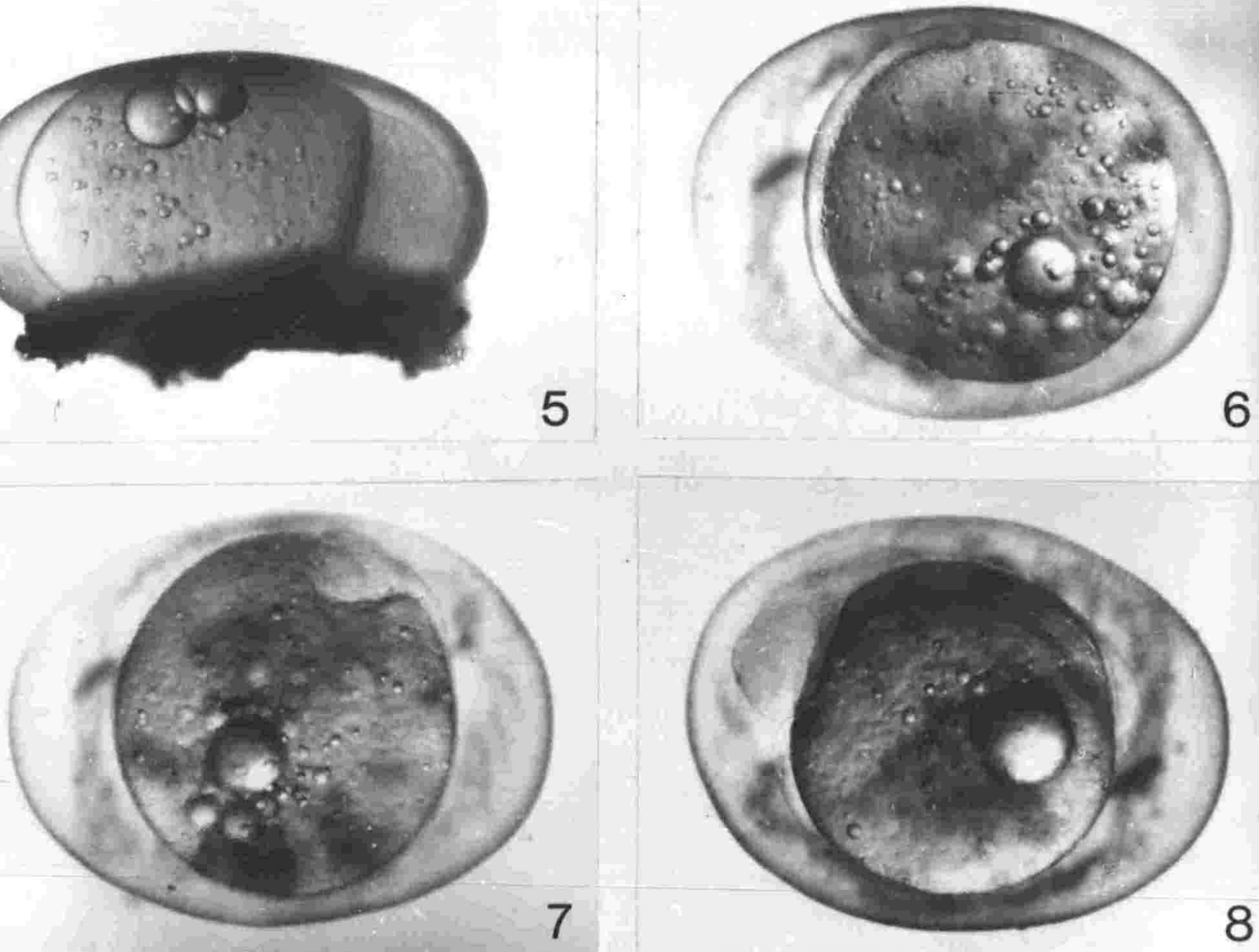
100.

Fig. 2. Trachelochismus melobesia. No.9: 5th day; 10:7th day; 11: 9 th day; 12 : 12 th day. 

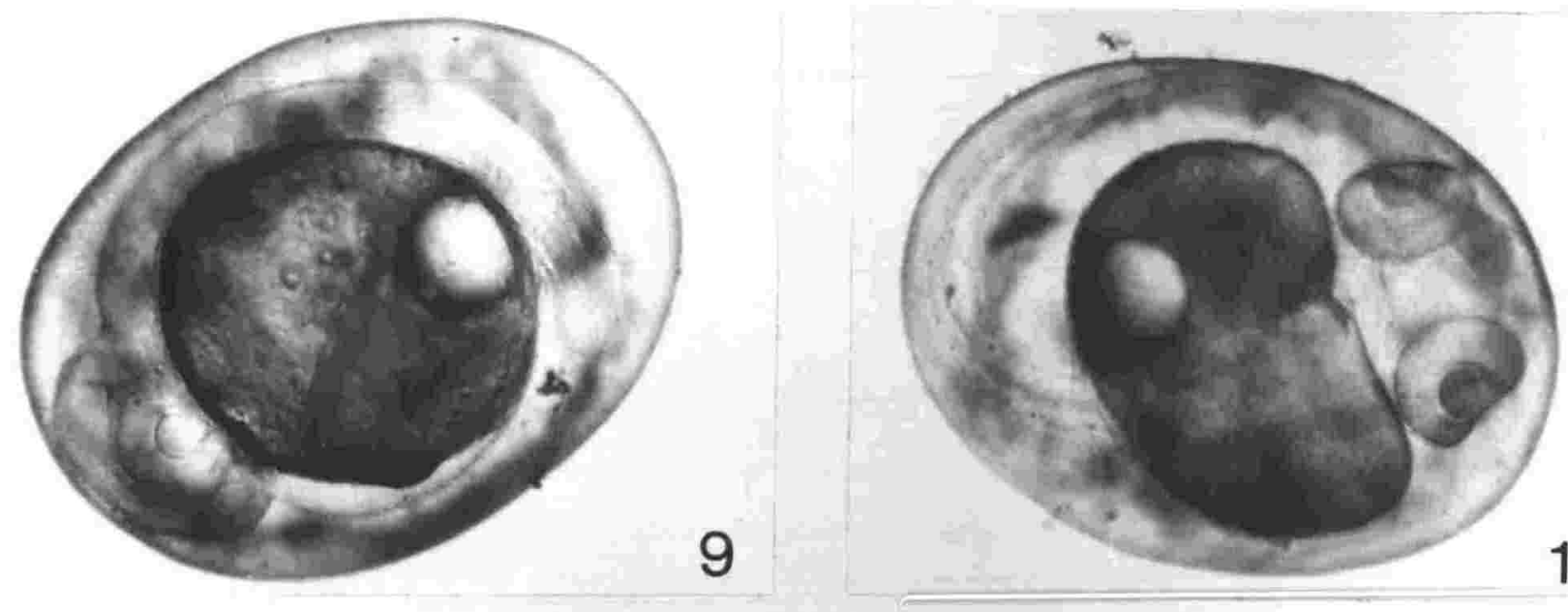

10



12 
Development of Tripterygion capito and $\underline{T}$. robustum

(Pisces: Tripterygiidae)

By J. G. Ruck

Zoology Department, Victoria University of Wellington.

Zoology Publications from Victoria University of

Wellington. No.63, issued 1973.

Abstract

In the Wellington area T. capito Jenyns, 1842 and T. robustum Clarke, 1879 spawn from July to October, depositing their eggs in clusters on the undersurface of rubble throughout the littoral zone. These clusters are attended by the male parent until hatching which under laboratory conditions occurs after 17 and 22 days respectively, in temperatures ranging from $11^{\circ} \mathrm{C}-13.5^{\circ} \mathrm{C}$. The egg development of both species is described.

The prolarvae of $\mathrm{T}$. capito average $4.90 \mathrm{~mm}$ standard length and possess yolk sacs of varying sizes each with 1-2 small oil droplets. Two large stellate melanophores lie above the gut. There are 16-26 stellate post-anal melanophores along the ventral mid-line, and 2-5 along the dorsal mid-line of the tail. The prolarvae of $\mathrm{T}$. robustum average $5.95 \mathrm{~mm}$ standard length and differ from T. Capito in having only 5-15 midventral melanophores.

\section{Introduction}

Tripterygion capito Jenyns, 1842 and T. robustum Clarke, 1879 are endemic tripterygiids abundant in areas of seaweed and rock throughout Wellington Harbour and along the shoreline of Cook Strait, New Zealand. Both species occur around all New zealand and are found throughout the intertidal zone. There are several other tripterygiid species in New 
Zealand but the systematics of these are at present confused.

Tripterygion capito is relatively small (maximum size $70 \mathrm{~mm}$ s.1.) and individuals are generally dark in colour although many specimens appear silver-grey with dark dorso-lateral bands from head to tail. Tripterygion robustum is largex (maximum size $105 \mathrm{~mm} \mathrm{s.1.}$ ) and characteristically has a blunt rounded forehead. The colour ranges from a uniform black to light green with irregular greenish-brown patches. The anal fin is often fringed with white.

This paper describes the egg and prolarval development of the two species. Graham (1939, 1953) has briefly outlined the egg and prolarval stages and spawning behaviour of $\underline{T}$. vaxium (Forster in Bloch and schneider, 1801) from New Zealand.

\section{Materials and Methods}

On 1.7.70 15 adult T. robustum were placed in an asbestos aquarium $(2.5 \times 0.38 \times 0.23 \mathrm{~m})$ at the Island Bay Marine Laboratory. Several stones and Haliotis (paua) shells were placed in the aquarium to provide shelter and objects on which to spawn. The tank was supplied with fresh running seawater and the fish wexe fed chopped liver and fish. On 8.7.70 freshly laid eggs were found on the undersurface of one of the Haliotis shells. These were attended by a male T. robustum (78 m s.1.) which, along with the eggs, was removed from the asbestos tank and placed in a small plastic aquarium ( $30 \times 28 \times 13 \mathrm{~cm}$ ). The water was changed daily and kept constantly aerated.

On 25.7.70 a similar system was set up using 10 adult T. capito, but no spawning occurred. However on 28.8 .70 an egg mass on a portion of clay pipe was collected from the Island Bay shore and kept in a plastic aquarium $(30 \times 28 \times 13 \mathrm{~cm}$ ), along with the adult male $T$. capito found attending the eggs. The watex was changed and aerated in the same manner as for $\mathrm{T}$. robustum.

Eggs of both species required for observation were easily removed from the substrate by sliding a scalpel or mounted needle beneath the egg and then pipetting the dislodged egg into a petri dish.

Development of these eggs was studied and sketches of the eggs and larvae were made using a camera lucida. An ocular micrometer was used for measuring eggs and larvae. Measurements of the larvae were based on those 
recommended by Hubbs and Lagler (1958), with the exception of head length, taken here as the distance from the tip of the snout to the back of the otic capsule. This is more definite than the opercular flap which is often not readily visible. Egg and larval terminology is based on that used by Rugh (1948), Balinsky (1965) and New (1966).

\section{Spawning and Development of T. capito}

In the Lyall Bay and Island Bay area near Wellington $T$. capito spawns from mid-July to late october. Egg masses are found from mid-tide to low tide levels in areas of rubble. The egg clusters are comparatively small measuring about $7 \times 4 \mathrm{~cm}$ and containing 2-3 hundred eggs. The eggs are laid close together forming flat, irregular shaped masses.

The egg clusters may contain groups of eggs at different stages of development, suggesting differing spawning times on the one substrate. In situ the eggs are colourless, pale pink or pale orange, the latter being associated mainly with the later "eyed egg" stages of development where the yolk is compact and the blood flow is copious. Being small and essentially transparent the egg ciusters may pass unnoticed by the casual observer. The pale pink eggs blend in with encrusting pink algae. Virtually all egg masses observed on the shore had an adult T. capito in attendance. On several occasions 2-3 adults were found close to the eggs. The male T. capito found with the eggs kept at the laboratory remained close to the eggs, moving from side to side and fanning the pectoral fins to create a curxent of water. This constant circulation apparently ensures a supply of oxygenated water to the eggs and helps in the removal of detritus that may otherwise attach to the egg surface.

\section{Development of the egg}

The egg is essentially spherical although slightly dorsoventrally depressed. The flattened base is covered with numerous fine, translucent, adhesive threads. The mean chorion diameter is $1.07 \mathrm{~mm}$ (100 eggs measured) and ranges from $1.00 \mathrm{~mm}-1.10 \mathrm{~mm}$. Early in development the yolk is spherical and averages $0.90 \mathrm{~mm}$ and contains $80-120$ small oil globules closely associated with 20-25 pigment spheres. No cluster was found with eggs earlier than the blastula stage and these were estimated 
to be about thirty hours old. Development to hatching (Fig. I, no's 1-9) took 17 days at a temperature ranging from $11^{\circ} \mathrm{C}-13.5^{\circ} \mathrm{C}$.

Thirty hours (Fig.1, no.1). The blastula is well formed and consists of a rounded cap of cells (blastoderm) overlying a deep blastocoel. Pigment spheres and associated oil droplets are distributed evenly throughout the yolk.

Two days (Fig.1, no.2). The blastodisc has spread half way around the yolk. The embryonic shield is poorly defined, appearing only as a thickened portion of the blastodisc.

Three days (Fig. I, no.3). The blastopore has closed. The optic vesicles are present but rudimentary. The embryo is deeply notched into the yolk, particularly in the head region, and the tail bud is flat. Several of the yolk pigment spheres have coalesced, as have some of the oil globules, effectively reducing slightly the number of each.

Fifth day (Fig.1, no.4). The pericardial cavity is well defined and the heart beats faintly, but there is no visible blood circulation. The brain is lobed and the ventricles pronounced. A cluster of small stellate melanophores lies anterior and posterior to each eye; the greatest number lie posterior to the eyes. The chorioid fissure is not closed. The auditory placodes appear as two small rings lateral to the hind brain. Behind each placode there is a group of about 10 small melanophores. Two more pigment clusters lie half way along the embryo, and several scattered melanophores are present in the tail bud. Pigment spheres and oil globules continue to coalesce.

Sixth day (Fig.1, no.5). The heart beats strongly and rapid circulation is visible in the dorsal and ventral blood vessels and the vitelline tributaries. Brain lobes have expanded laterally, especially the mesencephalon, and two otoliths are present within the otic vesicles. The tail extends well clear of the yolk and the gut is long and tubular. Several stellate melanophores surround the gut and extend in two rows along the ventral aspect of the tail. These melanophores are obscure and cannot be accurately counted. Ten to 15 stellate melanophores lie scattered over the yolk surface. Twenty to 30 oil droplets and 3-4 pigment spheres remain within the yolk. The relative position of the oil globules in the yolk varies according to the attitude of the egg because the oil floats to the uppermost portion of the yolk. 
Eighth day (Fig.1, no.6). The heart now lies slightly forward of the head. The chorioid fissure has closed and eye pigmentation is just visible. The general arrangement of the body pigment has altered very little. A large pigment patch is present at the posterior end of the gut, and a single row of 5 stellate melanophores runs along the ventral mid-line of the tail. Beginning directly above the anus 6 stellate melanophores run along the dorsal mid-line of the tail. Several melanophores are visible above the hind portion of the mesencephalon. All pigment spheres have disappeared from within the yolk. The tubular gut is longer.

Tenth day (Fig.l, no.7). The mesencephalon has expanded dorsally. The chorioid pigmentation is extensive although the lenses are still visible through it. The number of scattered melanophores present anterior and posterior to the eyes, above the gut and laterally within the tail is reduced. Two are present above the brain and 4 behind each otic vesicle. The melanophores along the dorsal mid-line are reduced to 3 or 4 while those on the ventral mid-line have increased to 11 or 12 . Rudimentary pectoral buds and fin folds are visible.

Twelfth day (Fig. 1, no.8). A further reduction in the number of melanophores above the gut, over the yolk surface, and anterior and posterior to the eyes has taken place. Those melanophores situated around the eyes adhere closely to the back of the eye and are somewhat obscured by the chorioid pigment. One or two melanophores lie along the lateral aspect of the body directly behind each pectoral bud.

Fouxteenth day (Fig.1, no.9). The yolk is considerably reduced, more dense and contains a single large oil globule and 13-15 smaller ones. The head is raised from the yolk revealing the chambers of the heart and the lower jaw. Pigmentation of the chorioid appears complete with many iridiophores present. The amount of body pigmentation varies. Many individuals have only those melanophores found above the gut and along the mid-ventral and mid-dorsal lines. Other specimens still possess the clusters behind each otic capsule and the 8 melanophores around the base of the brain and between the eyes. The mouth, external nares, pectoral fins and fin folds are well formed. By the 16th day (hatching) there is no pigment beneath the brain or behind the otic capsules. Just before hatching the embryo turns violently within the 
egg at frequent intervals. Eventually the tail penetrates the chorion, freeing the new prolarva.

Prolarva (Fig.1, no's 10 \& 11). Prolarval length on hatching ranges from $4.50 \mathrm{~mm}-5.25 \mathrm{~mm} \mathrm{s.1.} \mathrm{The} \mathrm{amount} \mathrm{of} \mathrm{yolk} \mathrm{present} \mathrm{varies,}$ being influenced by the degree of premature hatching caused by disturbance of the egg, such as by changing the water. The gut is long and convoluted. Above the gut, within the peritoneal layer, are two large melanophores. one directly anterior to the vent and the other above the hind portion of the liver. Some specimens also possess a melanophore beneath the gut and slightly anterior to the vent. The reduced yolk is bordered anteriorly by the heart and posteriorly by the liver and prominent green gallbladder. Sixteen to 26 (most commonly 21) stellate melanophores are present along the ventral mid-line behind the anus. Two to 5 dorsal melanophores are present in the tail. Two stellate pigment spots are situated either side of the anterior part of the yolk. An occasional specimen was found in which the melanophores around the brain and eyes and behind the otic capsules were still present, but these melanophores rapidly faded.

\section{Spawning and development of $T$. robustum}

In the Lyall Bay and Island Bay areas T. robustum spawns fxom early July to late october. Egg masses are found throughout the tidal zone beneath stones, broken clay pipes, sheets of asbestos and Haliotis shells. Egg clusters appear most abundant about the upper mid-tide level although many are found further up the tide, sometimas completely out of the water for several houxs during low tides. Several egg clusters were found in oyster shells located in spat collecting trays that were suspended from rafts 20 feet above the nearest portion of rocky seabed. The size of the masses range from $5 \mathrm{~cm}$ in diameter, with 1-2 hundred eggs, to about $12 \mathrm{~cm}$ in diameter with several thousand eggs. The eggs are laid close together forming flat irregularly arranged clusters.

The laxge egg masses contain groups of eggs at different stages of development, suggesting that like 2. capito, these clusters are the result of several different spawnings. The eggs are essentially colourless although collectively they appear pale green. The colour of the yolk (pale yellow) changes very little as development proceeds. Five separate egg masses were laid in the asbestos aquarium and in each 
case the male parent attended the eggs. The male T. robustum, like the parent T. capito, lay close to the eggs and created a current of water by movement of its anal and pectoral fins. Again this ensured a supply of oxygenated water and the removal of detritus. Egg masses kept without a male parent or adequate aeration suffered up to $90 \%$ mortality. These eggs also accumulated masses of minute debris which covered the entire chorion and obscured the embryo.

\section{Development of the egg}

The egg and yolk are completely spherical, and the mean chorion diameter of 80 eggs was $1.16 \mathrm{~mm}$ (range 1.12-1.23 mm). The yolk early in development is slightly granular, and contains 25-35 medium sized oil droplets closely associated with 15-25 pigment spheres. The eggs attach to the substrate by a mass of tendrils arising from one side of each egg. Each tendril consists of a matted series of fine filaments that arise from a single point on the chorion. These tendrils are larger than those of T. capito.

Development to hatching (Fig.2, no.1-12) took 22 days at a water temperature ranging from $11^{\circ} \mathrm{C}-13.5^{\circ} \mathrm{C}$.

Two hours (Fig.2, no.1). A single large mass of protoplasm appears at one end of the yolk.

Fifteen hours (Fig.2, no.2). One hundred and twentyeight angular cells form a low cap on one side of the yolk.

Thirty hours (Fig.2, no.3). The blastula is well formed and consists of a semi-circular mass of cells (blastoderm) overlying a wide blastocoel.

Fifty hours (Fig.2, no.4). The blastodisc covers two thirds of the yolk. The embryonic shield is clearly defined and lies slightly embedded in the yolk particularly in the anterior region.

Three days (Fig.2, no.5). The embryonic axis (neural keel and somite precursors) is obvious although still flattened against the yolk. Poorly defined optic vesicles are visible. Twelve to 20 large oil globules are present and the pigment spots have decreased in number (5-10). The blastopore is closed. 
Fourth day (Fig.2, no.6). The embryo is prominent above the yolk surface and encircles more than half of the yolk sac. The lenses are formed and have partially separated from the ectoderm. Eight myomeres lie well forward either side and Kupffer's vesicle has appeared dixectly beneath the tail bud. A group of tiny melanophores li.es anterior and posterior to each eye. A further two clusters are laterally positioned within the myomeres half way along the embryo.

Fifth day (Fig.2, no.7). The brain is lobed and the ventricles are visible. The auditory placodes appear as faint depressions behind the eyes. Approximately 15 stellate melarophores lie scattered over the surface of the yolk sac. The relative position of the melanophores is unchanged although there is an increase in the chromatophore number. A further group of melanophores has developed behind each otic vesicle.

Sixth day (Fig.2, no.8). The heart beats slowly and faintly but no blood flow is visible except very close to the heart. The pexicardial cavity has not yet expanded and the heart is embedded in the yolk directly beneath the head. The chorioid fissure has not closed. Each auditory placode appears as a double ringed structure. Four pairs of chromatophore groups are present, with one group anterior and another posterior to each eye, one behind each auditory placode and one two thirds back along the body of the embryo. The melanophores in these groups form a diffuse network making accurate counting impossible. The tail bud is still attached firmly to the yolk surface.

Seventh day. The heart beats regularly and strongly, and blood flows through the main vessels and across the yolk tributaries. The heart lies deep within the pericardial cavity slightly forward of the head. The sinus venosus is the only clearly visible chamber. The oil globules are reduced further (to 5-10), and all pigment spheres deep in the yolk have dispersed. The anterior myomeres are chevron shaped.

Eighth day. The yolk is dense and granular. The embryo alters position about once every minute.

Eleventh day (Fig.2, no.9). Pigmentation has appeared in the chorioid of the eye and the chorioid fissure has closed. The eyes have shifted laterally broadening the head and bringing the otic vesicles closer to the eyes. Several stellate melanophores are scattered above the brain and along the mid-ventral and mid-lateral aspects of the body 
and tail. The tail overlaps the head and the rudimentary pectoral buds are present.

Thirteenth day (Fig.2, no.10). Three otoliths lie within each otic vesicle. The chorioid pigment is darker although with transmitted light the lenses are still visible. Individual melanophores throughout the embryo are generally larger, although the size varies according to changes in light intensity. The melanophore clusters that were located two thirds back along the body have dispersed. Fewer pigment spots are present on the yolk surface. There is a large melanophore in the upper peritoneum slightly forward of the anus and 8 smallex ones above the gut in the region of the pectoral fins. The tail is long and passes over the head and returns to lie parallel with the body. Narrow fin folds are visible.

Seventeenth day (Fig.2, no.11). The head is lifted slightly from the yolk, exposing the chambers of the heart and the poorly developed lower jaw. The lenses are no longer visible through the eye pigmentation. The gallbladder j.s visible in the mid-gut region. Two to 8 oil globules are present within the yolk.

Twentieth day. The external nares appear as two shallow depressions anterior to the telencephalon. A row of 9-10 stellate melanophores runs along the mid-ventral line past the vent. Fin folds and pectoral fin buds are well developed.

Twenty second day (Fig. 2, no.12). The yolk is reduced considerably and the ventral side of the embryo faces upwards. Numerous iridiophores are present in the chorioid of the eye. A considerable reduction in the amount of pigment present around the brain, above the gut and in the lateral mid-line of the tail has taken place. Two to 3 chromatophores lie on the yolk surface. The mouth, external nares and pectoral fins are well defined. The embryo twists violently before hatching. The tail is flexed rapidly, as a result of which the chorion is ruptured and the prolarva is released. Immediately following this release the prolarvae swims to the surface.

Prolarva (Fig.3, no's 1 \& 2). Prolarval length on hatching ranges from $5.70 \mathrm{~mm}-6.10 \mathrm{~mm}$ s.l. All melanophores about the brain have faded. As with $\mathrm{T}$. capito the size of the yolk sac varies according to the degree of premature hatching. Three to 4 oil globules are present in the yolk. The upper gut peritoneum has two large melanophores, one slightly behind the yolk and the other slightly anterior to the anus. The yolk is bordered 
anteriorly by the heart and posteriorly by the liver and the prominent gallbladder. From 5-15 stellate melanophores are present along the ventral mid-line behind the anus and from 2-4 are situated along the dorsal midline of the tail. One or two melanophores lie beneath the pectoral fins on either side of the yolk. The prolarvae are positively phototropic. The average measurements of 25 prolarvae of each species are as follows :

\begin{tabular}{|c|c|c|}
\hline & T. capito & T. robustum \\
\hline Standard length (mm) & 4.90 & 5.95 \\
\hline Total length (mrn) & 5.17 & 6.20 \\
\hline Head length ( $\mathrm{mm}$ ) & 0.76 & 0.84 \\
\hline Eye length (mm) & 0.33 & 0.37 \\
\hline Snout to anus (mm) & 1.90 & 2.50 \\
\hline Mid-ventral melanophores & $21(16-26)$ & $7(5-15)$ \\
\hline Mid-dorsal melanophores & $2(2-5)$ & $2(2-4)$ \\
\hline Lateral yolk melanophores & 2 & $1(1-2)$ \\
\hline
\end{tabular}

\section{Acknowledgement}

I should like to thank Dr P.H.J. Castle, Department of Zoology Victoria University of Wellington, for his constructive criticism of this paper.

\section{Literature Cited}

Balinsky, B. I. 1965.

An introduction to embryology. Philadelphia and London:

S. B. Saunders Co., 673 pp., 443 fig., 23 tab.

Graham, D. H. 1939.

Breeding habits of the fishes of Otago Harbour and adjacent seas. Trans. Proc. R. Soc. N.Z., 69:361-372, 7 pl.

Graham, D. H. 1953.

Treasury of New Zealand fishes. Wellington: A. H. \& A. W. Reed, 404 pp., 153 fig.

Hubbs, C. L. \& Lagler, K. F. 1958.

Fishes of the great lakes region. Michigan: Cranbrook Institute of Science, 213 pp., 251 fig., 6 tab. 
New, D.A.T. 1966.

The culture of vertebrate embryos. London: Logos Pxess, Academic Press, 245 pp., 46 fig., 3 tab., 16 pl.

Rugh, R. 1948.

Experimental embryology. Minneapolis: Burgess Publishing Company, $481 \mathrm{pp}$.

J. G. Ruck, B.Sc. (Hons),

Department of Zoology,

Victoria University of Wellington,

P. O. Box 196,

Wellington, New Zealand. 
Fig. 1. Tripterygion capito. No.1: 30 hours; 2: 2nd day; 3: 3xd day; 4; 5th day, 5: 6th day; $6: 8$ th day; 7: 10th day; 8 : 12th day; 9: 14th day; 10 \& 11: prolarva, $4.51 \mathrm{~mm}$ s.1. 

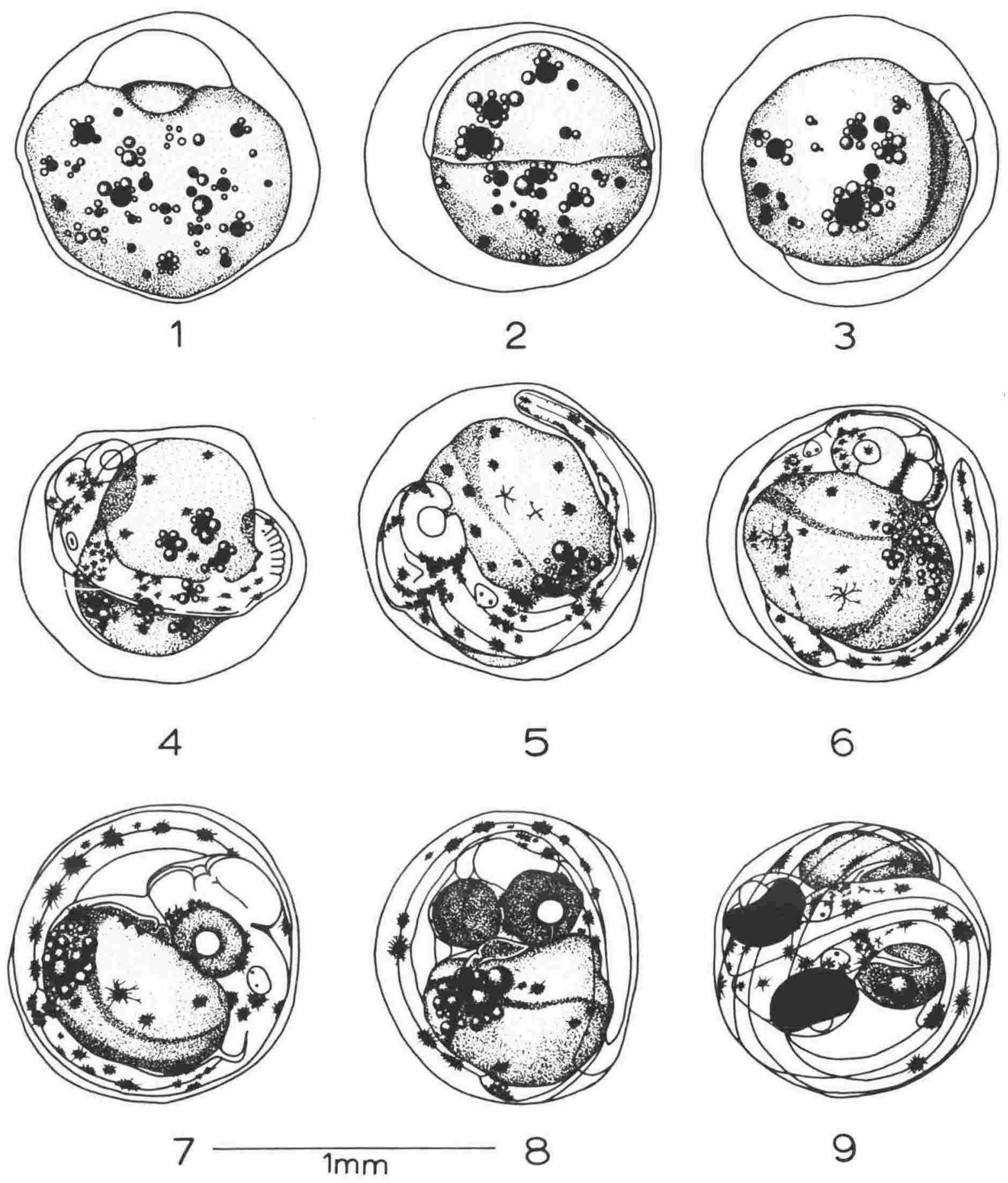

9

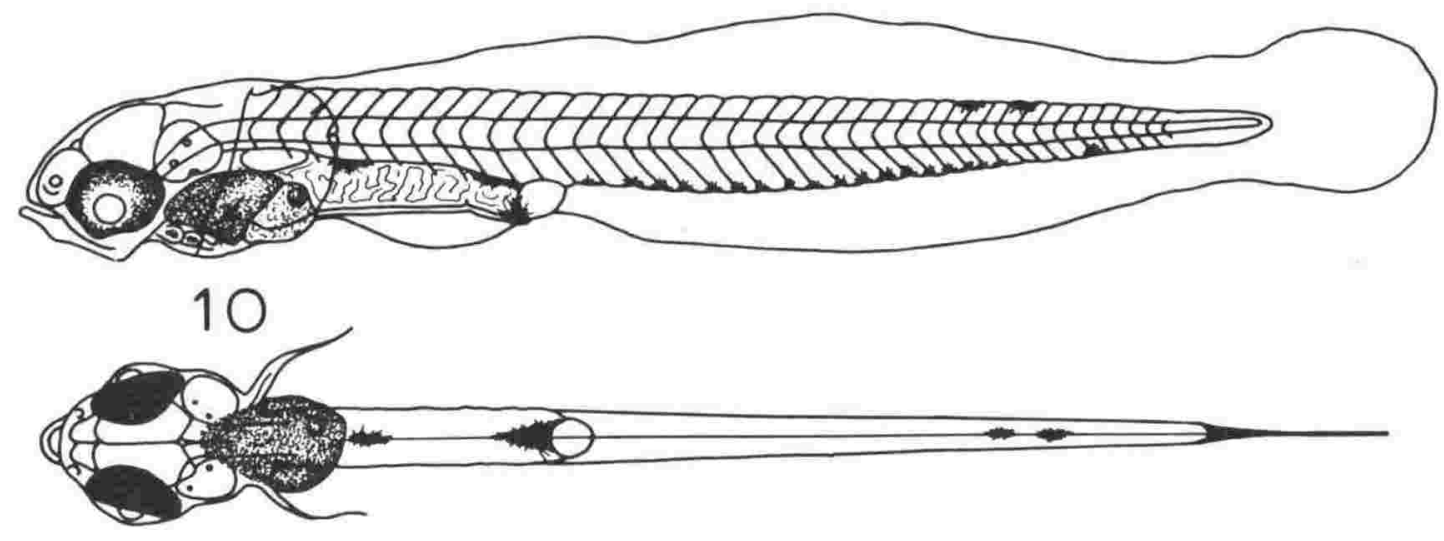

11 $1 \mathrm{~mm}$ 
113.

Fig. 2, Tripterygion robustum. No.1: 2 hours; 2: 15 hours; 3: 30 hours; 4: 50 hours; 5; 68 hours; 6: 4 th day; 7: 5 th day; 8 : 6 th day; 9 : 11th day; $10: 13$ th day; 11: 17 th day; 12 : 22nd day. 

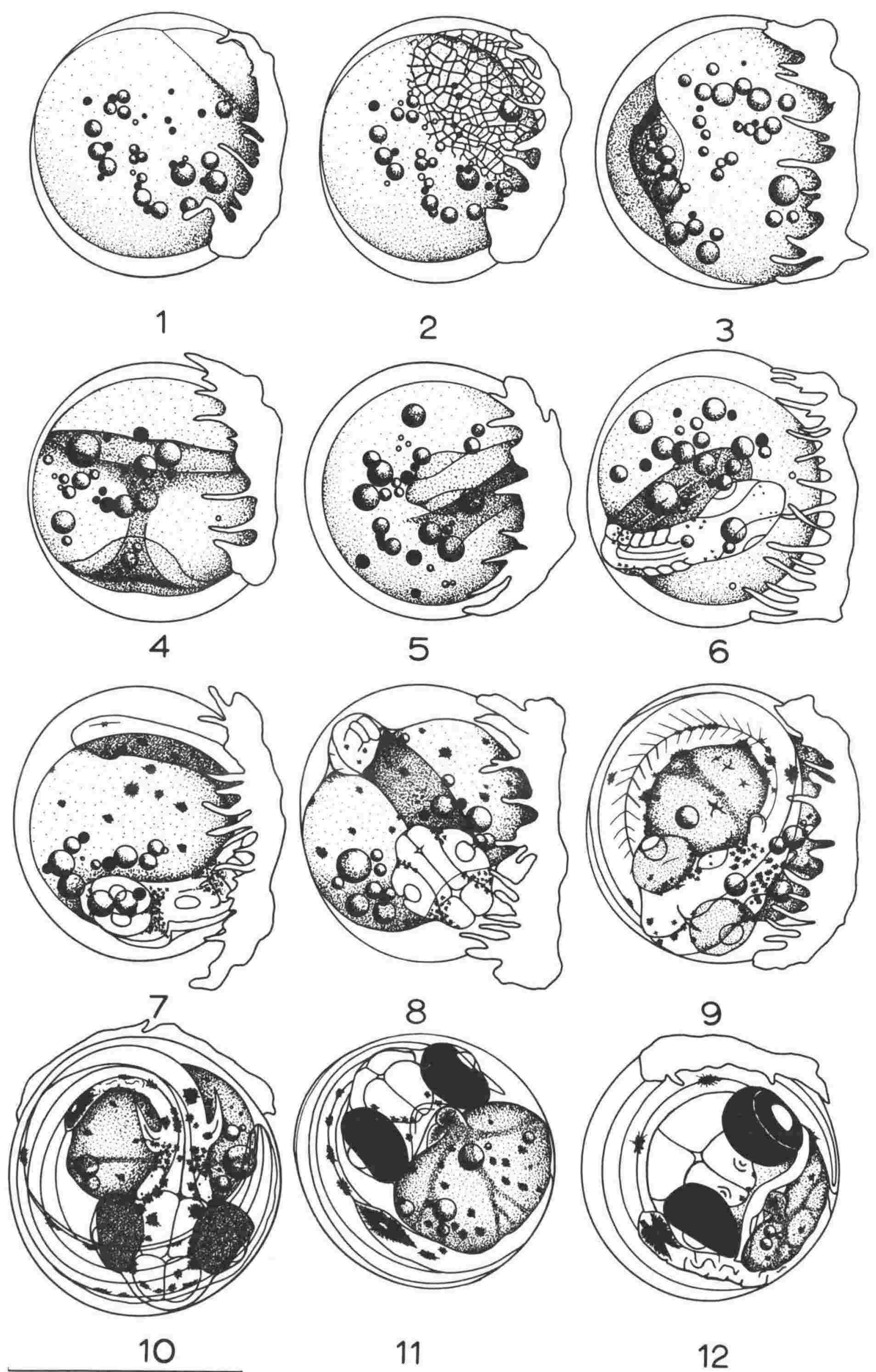
114.

Fig. 3. Tripterygion robustum. No's 1 \& 2: prolarva, $6.00 \mathrm{~mm}$ s.1. 


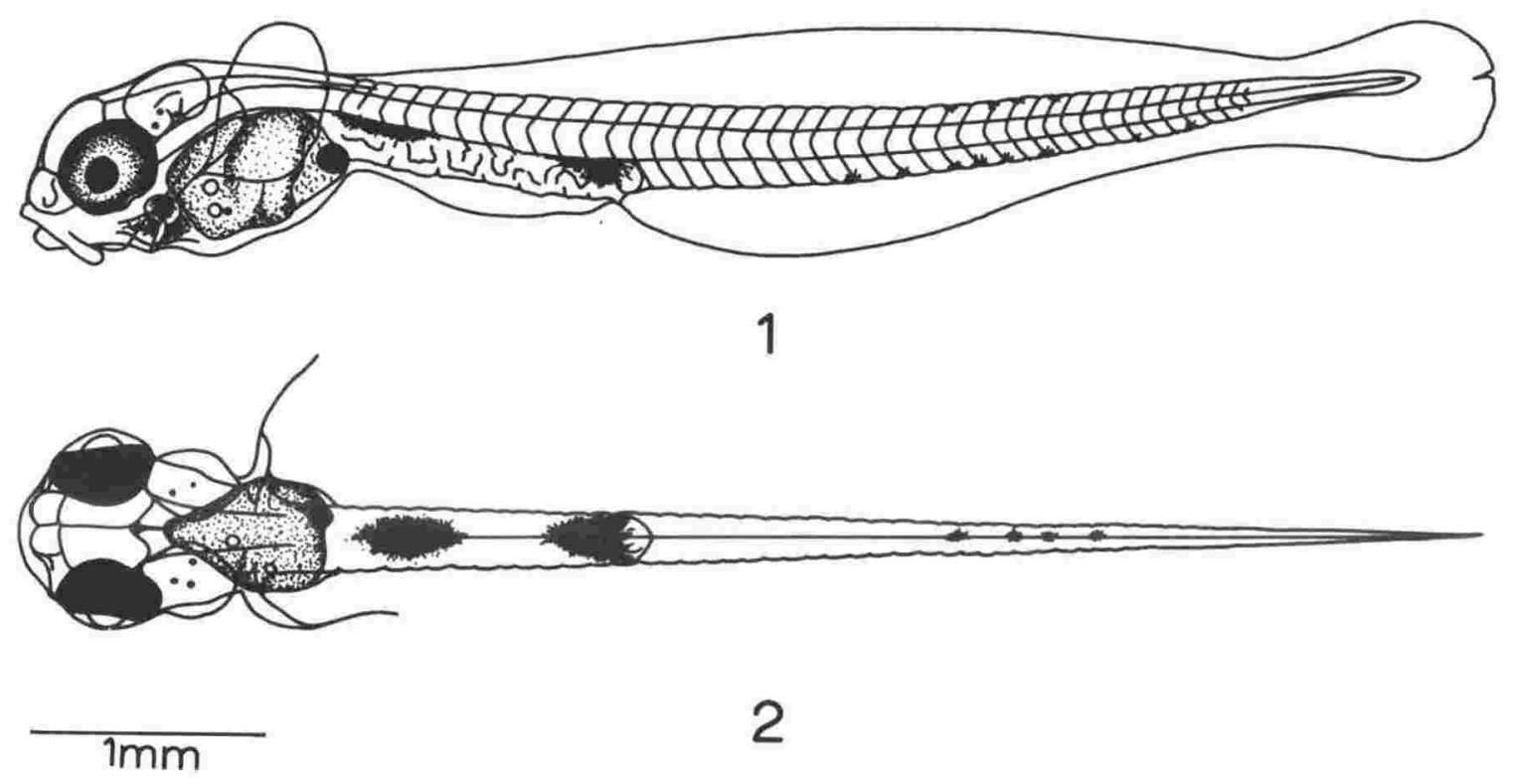


Development of the clingfishes, Diplocrepis puniceus, and Trachelochismus pinnulatus (Pisces: Gobiesocidae)

By J. G. Ruck

Zoology Department, Victoria University of Wellington. Zoology Publications from Victoria University of Wellington, No.64, issued 1973.

\section{Abstract}

In the Wellington area eggs of D. puniceus (Richardson, 1846) and T. pinnulatus (Forster in Bloch and Schneider, 1801) are laid from mid-winter to spring, in clusters on the undersurface of permanently tide-covered stones. Egg clusters of both species are attended by an adult until hatching. Under laboratory conditions hatching occurs for each species at 24 days in temperatures of about $11.5^{\circ} \mathrm{C}$.

The prolarva of D. puniceus is $5.00 \mathrm{~mm}-6.05 \mathrm{~mm}$ standard length and has a prominent pale mauve yolk sac with a single large oil globule. The upper parietal pexitoneum and the lower hind-brain are covered with conspicuous, stellate melanophores. Numerous melanophores are present on the myomeres of the trunk and peduncle. Caudal rays begin to develop by the 15th day.

The prolarva of T. pinnulatus ( $5.35 \mathrm{~mm}-6.10 \mathrm{~mm} \mathrm{~s} .1$.$) is similar$ to that of $\mathrm{D}$. puniceus but lacks the melanophores on the myomeres.

\section{Introduction}

Diplocrepis puniceus (Richardson, 1846) and Trachelochismus pinnulatus (Forster in Bloch and Schneider,1801) are endemic New Zealand species of the family Gobiesocidae. They are common throughout the midtide and low tide levels of the rocky shore around New zealand as is also the lumpfish Trachelochismus melobesia Phillipps, 1927. 
Diplocrepis puniceus is a relatively large clingfish with conspicuous coloration, attaining $100 \mathrm{~mm}$ standard length. The patterns and intensity of its body colour vary considerably in both male and female. In general, however, the dorsal surface is mottled with light and dark shades of green, contrasting with a pale yellow ventral surface. During the breeding season the males have an overall lilac tinge which is possibly a response to the deep purple colour of the egg clusters which they attend.

In T. pinnulatus the dorsal surface is light or dark green with longitudinal brown bands or brown spots and the ventral surface is pale yellow. Trachelochismus melobesia ( $\max$. size $30 \mathrm{~mm}$ s.l.) is similar to, but smaller than T. pinnulatus ( $\max$. size $72 \mathrm{~mm}$ s.1.) and has a reddishpurple patch on the dorsal surface which is lacking in T. pinnulatus. Diplocrepis puniceus is distinguished from T. pinnulatus and T. melobesia by its large horse-shoe shaped head and distinctive colour. The three are further distinguished by the following fin-ray counts (Briggs, 1955):

D. puniceus Dll $(10-11)$, A5 $(4-5), \mathrm{P}_{I} 23(23-24)$, C10

T. pinnulatus D8 (7-9), A6 (5-7), $\mathrm{P}_{I} 25(24-26), \mathrm{Cl} 2(11-12)$

T. melobesia D10 (9-11), A8 (7-8), PI $23(22-24), \mathrm{Cl} 2$

The present study describes the embryonic development and early larval growth of D. puniceus and T. pinnulatus. Comparable features of T. melobesia are described elsewhere (Ruck, 1971). The 1ife histoxy and general biology of T. pinnulatus were studied by Coakley (1964), and Graham (1939, 1953) has briefly described the egg and prolarval stages of D. puniceus.

\section{Materials and Methods}

On 21.7.70 an egg mass of D. puniceus (identification from description by Graham, 1939) was collected from the western shore of Lyall Bay on the north coast of cook strait. This was kept in a rectangular plastic container $(30 \times 28 \times 13 \mathrm{~cm})$ at the Island Bay Marine Laboratory. The water was changed daily and kept constantly aerated.

On hatching, prolarvae of D. puniceus were transferred to plastic containers $(12 \times 15 \mathrm{~cm})$ which had been sterilized by exposure to ultraviolet light. The water in each container was replaced daily with filtered and sterilized seawater. No artificial aeration was provided. Artemia ("brine shrimp") nauplii were fed to the larvae as soon as most of the 
yolk sac had been absorbed. The last larva died on the 15th day after hatching. During the 15 days several larvae were anaesthetised with Sandoz MS222 and sketches were made.

Larvae of $\mathrm{D}$. puniceus larger than $5.50 \mathrm{~mm}$ were also obtained from the plankton approximately $100 \mathrm{~m}$ off-shore at Island Bay and Lyall Bay, using a plankton net of standard conical design wi.th a $57 \mathrm{~cm}$ diameter opening and a mesh size of 500 microns.

Early in July, 1970,10 adult T. pinnulatus were collected from

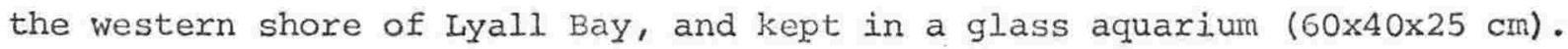
Stones, broken clay piping and empty Haliotis (paua) shells were placed in the aquarium to provide shelter and areas on which to spawn. The tank was supplied with fresh running seawater and the fish were fed chopped fish and beef liver. On 28.7.70 freshly deposited eggs were found on the undersurface of a portion of the clay pipe. These eggs wexe attended by a male T. pinnulatus ( $61 \mathrm{~mm}$ s.1.) which, along wi.th the egg mass, was transferred to a plastic container $(30 \times 28 \times 13 \mathrm{~cm})$. The water was changed daily and supplied continuously with air.

The egg masses of both species adhered closely to the substrate and hence eggs required for observation were very difficult to remove without damage to the egg membrane. Limited success was obtained by sliding a sharp scalpel between the egg and the substrate and then pipetting the dislodged egg into a petri dish. Development of the eggs of $\mathrm{D}$. puniceus and $T$. pinnulatus were studied and sketches of the eggs and larvae were made with a camera lucida. An ocular micrometer was used for measuring eggs and larvae. Measurements of the larvae are those recommended by Hubbs and Lagler (1958:24-26), with the exception of head length, taken here as the distance from the snout to the posterior margin of the otic capsule. In the prolarva the otic capsule provides a more positive point of reference than the opexculum. Descriptive terminology of eggs and prolarvae follows that used by Rugh (1948), Balinsky (1965) and New (1966). 


\section{Spawning and Development of D. puniceus}

In the Lyall. Bay area D. puniceus spawns from early July to late September. The eggs are deposited on the undersurfaces of large stones and are in close contact, forming flat ixregularly shaped egg masses which remain covered with water at low tide. Typically the egg masses are relatively large; one kept in the laboratory measured $13 \times 5 \mathrm{~cm}$ and contained approximately 2,400 eggs. Each egg mass contains sub-groups, each with up to 300 eggs, which differ slightly in colour, ranging from deep purple to pale mauve. The deep purple eggs appear crirnson when yiewed under the binocular microscope. The variation in colour between sub-groups is mainly a result of the progressive depletion of the yolk supply in the eggs of each sub-group as development advances. This suggests that the eggs of adjacent sub-groups within the same mass are deposited at different times. The pale and small-yolked eggs, which show well-developed eyes, are the most advanced in development and are found mainly in the centre of the egg mass. In some sub-groups, however, the well-developed eggs retain deep purple yolks.

The male collected with the egg mass from Lyall Bay remained with the eggs constantly. It did not eat food offered. It swam backwards and forwards across the eggs, maintaining a constant flow of water over the egg mass. This helped to remove detritus from the egg mexabranes, and made observation of the embryo through the chorion relatively easy. By comparison the chorion of eggs kept separate from the male soon became covered in foreign particles to such a degree that the embryo and yolk within were very difficult to see.

\section{Development of the Egg}

Most of the eggs within the clusters are laid so close together that they impinge on each other and so deform the chorion (Fig.1, no.1). The consequent irregularities in the shape of most eggs make accurate measurement of the egg diameter difficult. Eggs that make no contact with their neighbours, however, are spherical when viewed dorsally, and have a mean diameter of $1.80 \mathrm{~mm}$. The yolk is sub-sphexical and in a standard sample of 100 eggs has a mean diameter of $1.30 \mathrm{~mm}$. During early development the 
yolk is dark purple and contains a single large oil globule (mean diameter $0.45 \mathrm{~mm}$ ) and 20-30 smaller ones. The eggs are dorso-ventrally depressed and are attached to the substrate by a flattened adhesive base.

Development to hatching (Fig.1, no's 1-12) takes 24 days at $11.5^{\circ} \mathrm{C}$. No eggs were found earlier than the gastrulation stage, and these were estimated to be nearly two days old.

Second day (Fig.1, no.1). At this stage the blastodisc has spread halfway around the yolk. Epiboly is not obvious, except for a slight thickening of the germ ring.

Third day (Fig.1, no's 2 \& 3). The embryonic shield is well defined and lies deeply notched into the yolk. The blastodisc continues to expand over the yolk, covering about two-thirds of the yolk surface. Separated eggs retain the ring-like shallow depressions that were produced by contact with other eggs.

Fourth day (Fig.1, no.4). The blastopore is reduced to a small opening through which the yolk bulges. The neural keel (future central nervous systen) is well defined, encircling almost half of the yolk. The optic vesicles are present but rudimentary.

Fifth day. The three main divisions of the brain are distinguishable. The closing of the blastopore is complete. Three to four myomere blocks are present on either side of the neural keel.

Sixth day (Fig.1, no.5). The embryo has increased in length and is well defined. The optic cups can be seen surrounding the spherical lens tissue. Approximately 13 pairs of myomeres are present, arising posterior to the faint outlines of the auditory placodes. Kupffer's vesicle is present as two small sacs beneath the tail bud.

Seventh day (Fig.1, no.6). The lobes of the brain are well defined and from the dorsal aspect the ventricles of the prosencephalon, mesencephalon and the rhombencephalon are conspicuous. Each ventricle is covered by a thin roof. The optic cups enclose the lenses but as yet the chorioid fissure has not closed. The anterior myomeres are chevron-shaped, and the tail bud has begun to lift clear of the yolk.

Ninth day (Fig.1, no's $7 \& 8$ ). There is a distinct pericardial. cavity beneath, and extending forward of, the head. The heart beats faintly, but there is no visible circulation, and the crimson-purple of the 
yolk obscures any sign of blood-island formation. The head is expanded laterally, and the brain ventricles, especially that of the mesencephalon, are relatively large. Pigmentation of the chorioid of the eye has begun. The gut is tubular, and there are scattered, stellate melanophores in the trunk muscle directly above it.

Eleventh day (Fig.1, no.9). The heart beats regularly and strongly, and a flow of blood through the dorsal and ventral blood vessels is obvious. The sinus venosus is pronounced, lying within the pericardial cavity slightly forward of the head, and receiving blood from the large vitelline vessels. The chorioid fissure has closed completely and appears as a faint white line on the ventral aspect of the eye. Chorioid pigmentation has increased. The brain lobes are more rounded and two otoliths are present in each otic vesicle. The tail bud is less rounded and extends well clear of the yolk, and the upper parietal peritoneum is covered by scattered melanophores. A single large oil globule is present in the yolk.

Fourteenth day (Fig.1, no.10). The head is broad and has lifted from the yolk, exposing the chambers of the heart and the rudimentary lower jaw. The eyes are prominent and the lenses can still be seen through the chorioid pigment. Pectoral fin buds are present and the tail is turned to lie parallel with the body.

Sixteenth day (Fig.1, no.11). The external olfactory pits appear as shallow depressions anterior to the telencephalon. The tail overlaps the head and there is an increase in the peritoneal and segmental pigmentation. The peritoneal melanophores extend forward beneath the myelencephalon. The embryo changes position frequently.

Twenty-fourth day (Fig.1, no.12). The yolk is reduced considerably and the ventral aspect of the embryo faces upwards. Pigmentation of the eye appears complete with many small iridiophores present. The embryo is cramped within the chorion and the tail completely overlaps the head and turns on itself. The mouth, olfactory bulbs and pectoral fins are well formed. The gut is large and convoluted. The liver lies posterior to the yolk and contains a green spherical gallbladder. Just prior to hatching the embryo becomes agitated and the tail begins to flex. As a result, the chorion is ruptured and the prolarva is released. 
Prolarva (Fig.2, no's $1 \& 2$ ). Prolarval length on hatching ranges from $5.00 \mathrm{~mm}-6.05 \mathrm{~mm} \mathrm{s.1}$. There is considerable variation in the amount of yolk present in each prolarva immediately after hatching. Large amounts of yolk in some prolarvae may be accounted for by premature hatching, induced by disturbance. The yolk contains a single reduced oil globule. The heart is prominent and lies on the anterior yolk margin. The gut is long and convoluted and extends beyond the mid-length of the body. Numerous melanophores (30-50) are present on the myomeres of the body and tail, the first of which is placed behind the pectoral fin base; this series extends to the fifth myomere past the vent. Occasional specimens have as few as 6 melanophores on the myomeres. A yellow tinge is present deep in the muscle tissue directly beneath the melanophores of the body and tail. Numerous melanophores line the upper parietal peritoneum. The head is broad and blunt, and there are two clusters of stellate melanophores beneath the myelencephalon. The prolarvae are positively phototropic.

Eight day larva (Fig.2, no's $3 \& 4$ ). $6.20 \mathrm{~mm} \mathrm{s.1.} \mathrm{The} \mathrm{yolk}$ is almost completely absorbed and the oil globule is very small. Pigmentation is unaltered except for the development of several melanophores at the base of each pectoral fin. A yellow tinge surrounds the base of the brain, particularly the myelencephalon, and extends posteriorly within the myomeres above the gut.

\section{Fifteenth day larva (Fig.2, no's $5 \& 6$ ). $7.70 \mathrm{~mm} \mathrm{s.1.} \mathrm{The}$} overall shape of the larva has altered slightly. There is an increase in the depth of the tail and the relative size of the gut. The pigment pattern is unchanged. Sucker buds are visible at the base of the pectoral fin. Myomeres have the double chevron pattern and the notochord is slightly upturned in the tail. Six rudimentary caudal rays are present. Larval and prolarval measurements in millimetres are as follows:

$\begin{array}{lllc} & \frac{\text { Prolarvae }}{25} & \frac{10 \text { days }}{25} & \frac{15 \text { days }}{1} \\ \text { Number of fish } & 5.67(5.40-6.60) & 7.12(6.95-7.30) & 7.90 \\ \text { Total length (mm) } & 5.28(5.00-6.05) & 6.70(6.55-6.90) & 7.70 \\ \text { Standard length (mm) } & 1.10(1.00-1.15) & 1.30(1.25-1.40) & 1.70 \\ \text { Head length (mm) } & 0.52(0.50-0.54) & 0.54(0.51-0.56) & 0.65 \\ \text { Eye length (mm) } & 3.32(3.20-3.51) & 4.41(4.15-4.55) & 5.00 \\ \text { Snout to vent (mm) } & 1.15(1.00-1.21) & 1.02(0.90-1.10) & 1.35 \\ \text { Greatest depth (mm) } & & & \end{array}$


Spawning and Development of T. pinnulatus

In the Lyall Bay area T. pinnulatus begins spawning early in July and continues through to mid-october. South of cook strait spawning occurs later. Along the Canterbury coast north of Christchurch the "Earliest records of spawning in the field were made late in August in both 1962 and 1963" (Coakley 1964). Egg masses are found beneath large and small stones, Haliotis shells and other debris which offer secure shelter and remain covered with water at low tide. The egg mass from which this description is made was small $(4 \times 1.5 \mathrm{~cm})$ and contained 198 eggs. Typically, however, egg masses are large (up to $10 \times 7 \mathrm{~cm}$ ) and contain as many as 1500 eggs. Coakley (1964) observed that the earliest spent females were the largest, indicating that the larger fish spawn early in the season. The eggs are laid close together forming flat, irregularshaped masses. The larger egg clusters always contain groups of eggs which differ appreciably in colour, that is white, orange, pale pink, crimson-red, or pale yellow. There is little intergrading of these colours. Egg sub-groups differing in colour in this way may be at similar stages of development. The variation in colour, unlike that of $\underline{D}$. puniceus, does not represent the progressive depletion of the yolk supply in the eggs as development advances, although as time proceeds there is a paling of the colour of each sub-group. It suggests, rather, that the different coloured sub-groups are laid on the same substrate by different females, and that the variation in yolk colour is due to some biochenical difference between each contributing female. The male T. pinnulatus found with the eggs in the aquarium remained close to them even when alternative shelter was provided. It lay over the eggs, fanning the pectoral and caudal fins, thus maintaining a constant flow of water over the surface of the egg cluster. This current again helped to prevent the accumulation of detritus on the egg membrane. Well over $90 \%$ of the egg masses observed on the shore had an adult T. pinnulatus in attendance. Coakley (1964) showed, by dissection, that the majority of adults found close to the egg clusters were males, but only one attendant adult was sexed in the present study. 


\section{Development of the egg and prolarva}

The eggs are oval (mean dimensions of a standard sample of 100 are $1.81 \times 1.48 \mathrm{~mm})$, dorsoventrally depressed and attached to the substrate by a flattened adhesive base. At an early stage of development the yolk is central and subspherical and has a mean diameter of $1.30 \mathrm{~mm}$. Development to hatching (Fig. 3, no's 1-11) takes 24 days at $11.5^{\circ} \mathrm{C}$. No eggs were found earlier than the gastrulation stage, and these were estimated to be approximately two days old.

Second day (Fig.3, no.1). The blastodisc has spread half way around the yolk. Epiboly is not obvious. There are six main oil globules and about 150 smaller ones present in the yolk.

Third day (Fig.3, no.2). The blastodisc continues to expand over the yolk, covering about two-thirds of the yolk surface. The embryonic shield is present as a thickened stxip of the blastodisc.

Fourth day. The blastopore is reduced to a small opening bordered by the thickened germ ring. The narrow embryonic shield lies notched into the yolk. Many of the small oil globules have coalesced to form large globules.

Fifth day (Fig. 3, no.3). The closing of the blastopore is complete. The optic vesicles are present but rudimentary. The embryo is still deeply notched into the yolk, particularly in the head region, and the tail is flat.

Seventh day (Fig.3, no.4). The embryo has increased in length, encircling more than half of the yolk. There is a distinct pericardial. cavity anterior to the head. The three main divisions of the brain are distinguishable. The lens tissue is separate from the ectoderm. Approximately 11 pairs of myomeres are present, arising posterior to the faint outlines of the auditory placodes. Kupffer's vesicle lies beneath the developing tail bua.

Eighth day (Fig.3, no's 5 \& 6). The brain, from the dorsal aspect, shows clearly defined ventricles, each covered by a thin roof. The optic cups surround the spherical lens tissue, and the chorioid fissure has not yet closed. The anterior myomeres are chevron-shaped, and the tail bud begins to lift clear of the yolk. 
Tenth day (Fig.3, no.7). The heartbeat is regular, sending blood slowly through the major blood vessels. The head has expanded laterally, and the brain ventricles, especially that of the mesencephalon, have increased in size. The chorioid is slightly pigmented and the fissure has closed. Two small otoliths are present in each round otic vesicle. The gut is thin and tubular.

Fourteenth day. The head is broad and has lifted from the yolk, exposing the chambers of the heart and the developing lower jaw. The sinus venosus is a thin expanded sac lying within the pericardial cavity forward of the head, and receiving blood from the large vitelline vessels. The brain lobes are rounded and larger. The chorioid fissure appears as a faint white line on the ventral aspect of the eye. The eyes are prominent and the lenses can still be seen through the chorioid pigment. The upper parietal peritoneum has scattered melanophores. Pectoral fin buds are present and the tail has turned to lie parallel with the head and trunk. A single large oil globule and 10 smaller ones are present in the yolk.

Sixteenth day (Fig.3, no.8). Very little change has taken place, except that the jaws appear more definite and slightly fleshy.

Twentieth day (Fig.3, no's $9 \& 10$ ). The olfactory pits appear as shallow depressions anterior to the telencephalon. The tail overlaps the head and there is an increase in the number of peritoneal melanophores. The embryo alters position frequently. A single large oil globule remains in the yolk.

Twenty-fourth day (Fig.3, no.11). The yolk is reduced considerably and the ventral aspect of the embryo faces upwards. Pigmentation of the chorioid appears complete, the silvery appearance of the eye being due to the presence of iridiophores. The embryo is cramped within the egg and the tail completely overlaps the head and turns on itself. The mouth, olfactory bulbs and pectoral fins are well formed. The gut is long and convoluted. The liver and the green spherical gall bladder lie behind the yolk. The upper peritoneal pigment is arranged approximately as two rows of 10 large stellate melanophores, extending from above the liver to the vent. In some individuals two rows of 3-4 stellate melanophores are present on the lower parietal peritoneum extending back from the yolk. Just prior to hatching the embryo becomes active and begins to flex its 
tail. As a result, the chorion is ruptured and the prolarva is released.

Prolarva (Fig.4, no's 1 \& 2). Prolarval length on hatching ranges from $5.35 \mathrm{~mm}-6.10 \mathrm{~mm}$. Like D. puniceus there was some variation in the amount of yolk present in each prolarva immediately after hatching. The yolk contains a single reduced oil globule, and the heart lies on the anterior margin of the yolk sac. The gut is long and convoluted and extends past the mid-length of the body. Upper peritoneal pigmentation is scattered and extends from above the liver to the vent. Some individuals retain the lower peritoneal pigmentation. A yellow tinge lies within the muscles directly above the peritoneal pigment, and extends beneath the otic capsules and the hind-brain. Two stellate melanophores are present beneath each pectoral fin, but no pigment is present on the head.

The measurements of prolarvae are as follows:

$\begin{array}{lc}\text { Number of fish } & 23 \\ \text { Standard length (mm) } & 5.70(5.35-6.10) \\ \text { Head length (mm) } & 1.04(0.95-1.10) \\ \text { Eye length (mm) } & 0.43(0.40-0.45) \\ \text { Snout to vent (mm) } & 3.43(3.20-3.60) \\ \text { Greatest depth (mm) } & 1.02(0.80-1.20)\end{array}$

Acknowledgement

I should like to thank Dr P.H.J. Castle, Department of Zoology, Victoria University of Wellington, for his constructive criticism of this paper. 


\section{Literature Cited}

Balinsky, B. I. 1965.

An introduction to embryology. Philadelphia and London:

W. B. Saunders Co., 673 pp., 443 fig., 23 tab.

Briggs, J. C. 1955.

A monograph of the clingfishes (Order xenopterygii) Stanford Ichthyol. Bull, , 6:224 pp., 114 fig., 1 tab.

Coakley, A. 1964.

Life history and general biology of Trachelochismus pinnulatus (Forstex) (Order Xenopterygii). Unpublished M.Sc. thesis, University of Canterbury New Zealand.

Graham, D. H. 1939.

Breeding habits of the fishes of otago Harbour and adjacent seas. Trans. Proc. R. Soc. N.z., 69: 361-372, $7 \mathrm{pl}$.

Graham, D. H. 1953.

Treasury of New Zealand fishes. Wellington: A. H. \& A. W. Reed, 404 pp. , 153 fig.

Hubbs, C. L. \& Lagler, K. F. 1958.

Fishes of the great lakes region. Michigan: Cranbook Institute of Science, 213 pp., 251. fig., 6 tab.

New, D.A.T. 1966.

The culture of vertebrate embryos. London: Logos Press Limited, 245 pp., 46 fig., 16 pl.

Ruck, J. G. 1971.

Development of the lumpfish Trachelochisnus melobesia (Pisces: Gobiesocidae). Zool. Publs Vict. Univ., Wellington. 57: 1-9, 4 fig.

Rugh, R. 1948 .

Experimental embryology. Minneapolis: Burgess Publishing Company, $481 \mathrm{pp}$. 
127.

J. G. Ruck, B.SC. (Hons),

Department of Zoology,

Victoria University of Wellington,

P. O. Box 196,

Wellington, New Zealand. 
128.

Fig.1. Diplocrepis puniceus. No.1: 2nd day, 2 \& 3 : 3 rd day; 4: 4 th day; $5: 6$ th day; $6: 7$ th day; 7 \& 8: 9th day; 9: 11th day; 10: 14th day; 11: 16th day: 12: 24th day. 

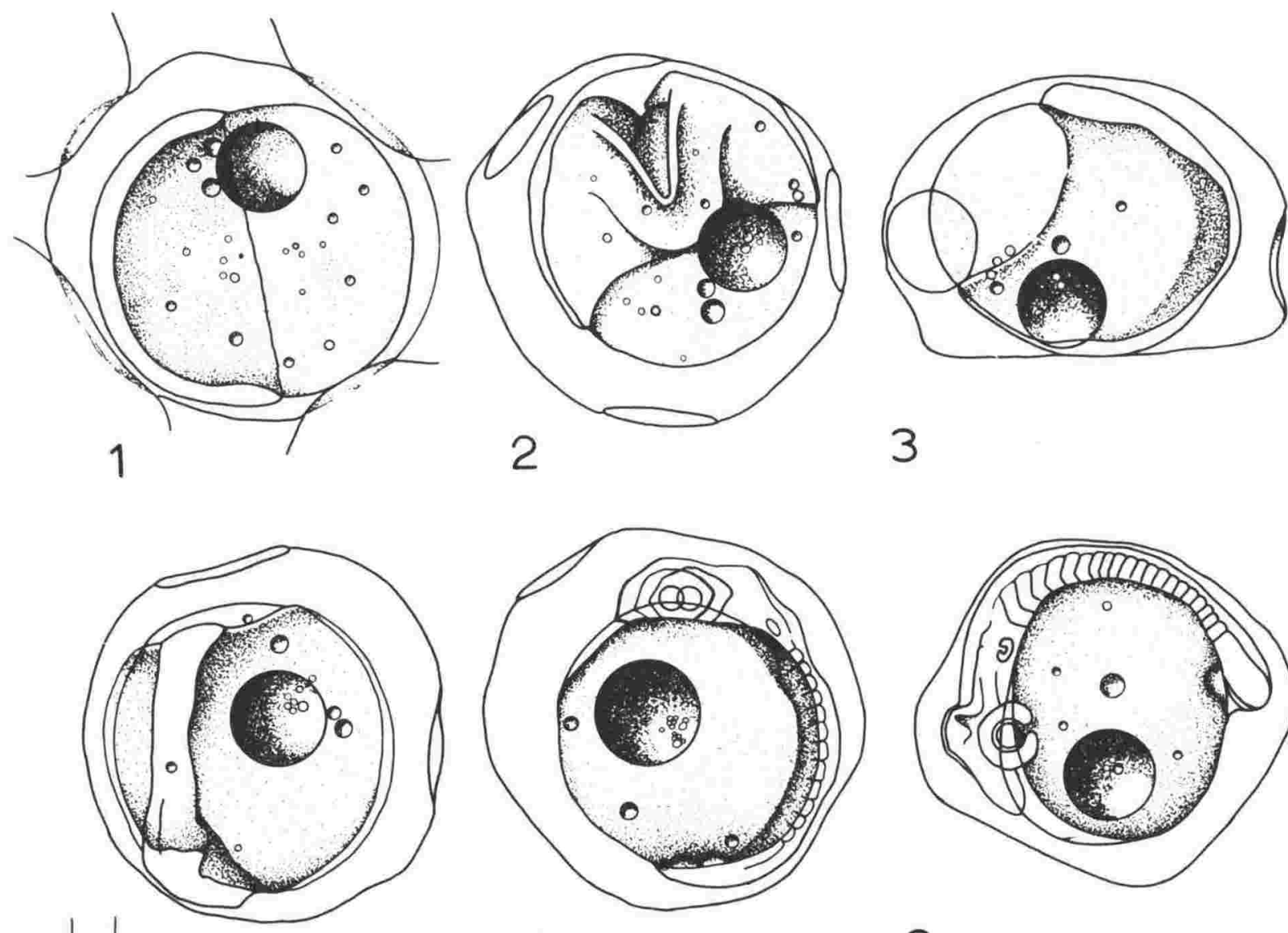

b) 4
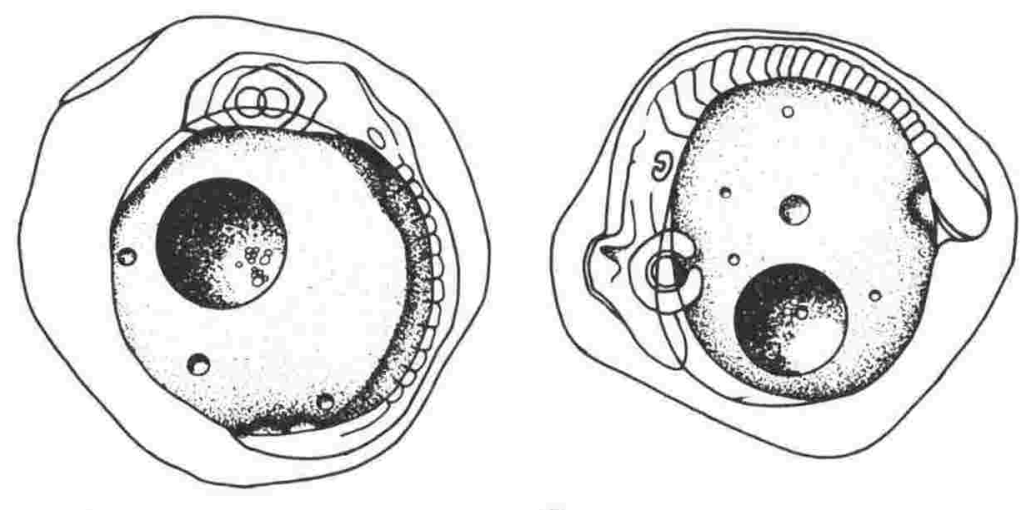

6
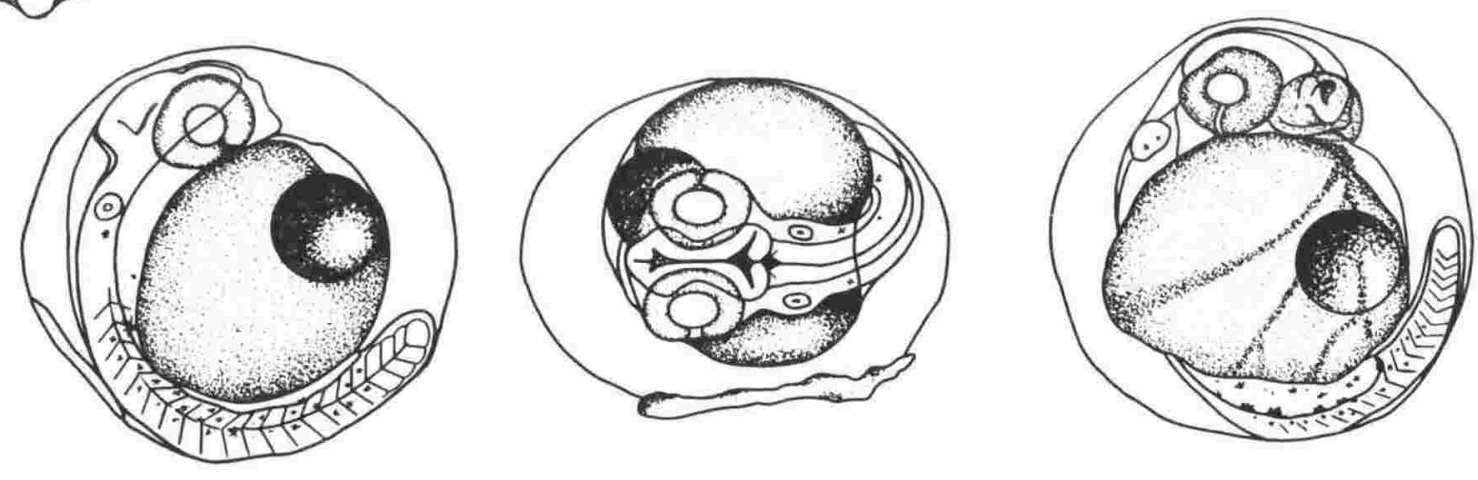

$$
7
$$

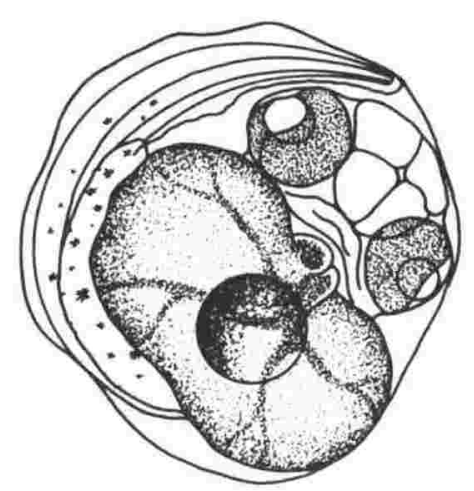

10

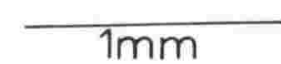

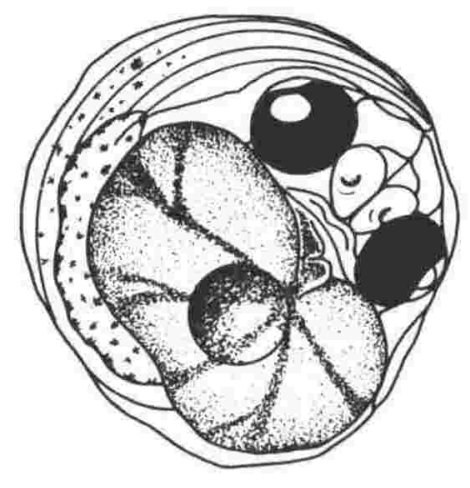

11
9

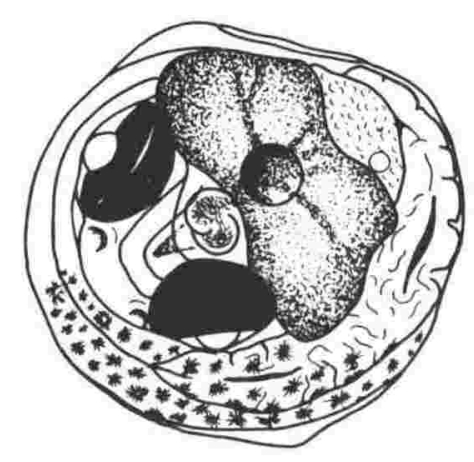

12 
129.

Fig.2. Diplocrepis puniceus. No's 1 \& 2: prolarva, $6.05 \mathrm{~mm}$ s.1.; 3 \& 4: 8 day old larva, $6.20 \mathrm{~mm}$ s.1, ; 5 \& 6: 15 day old larva, $7.70 \mathrm{~mm}$ s.1. 

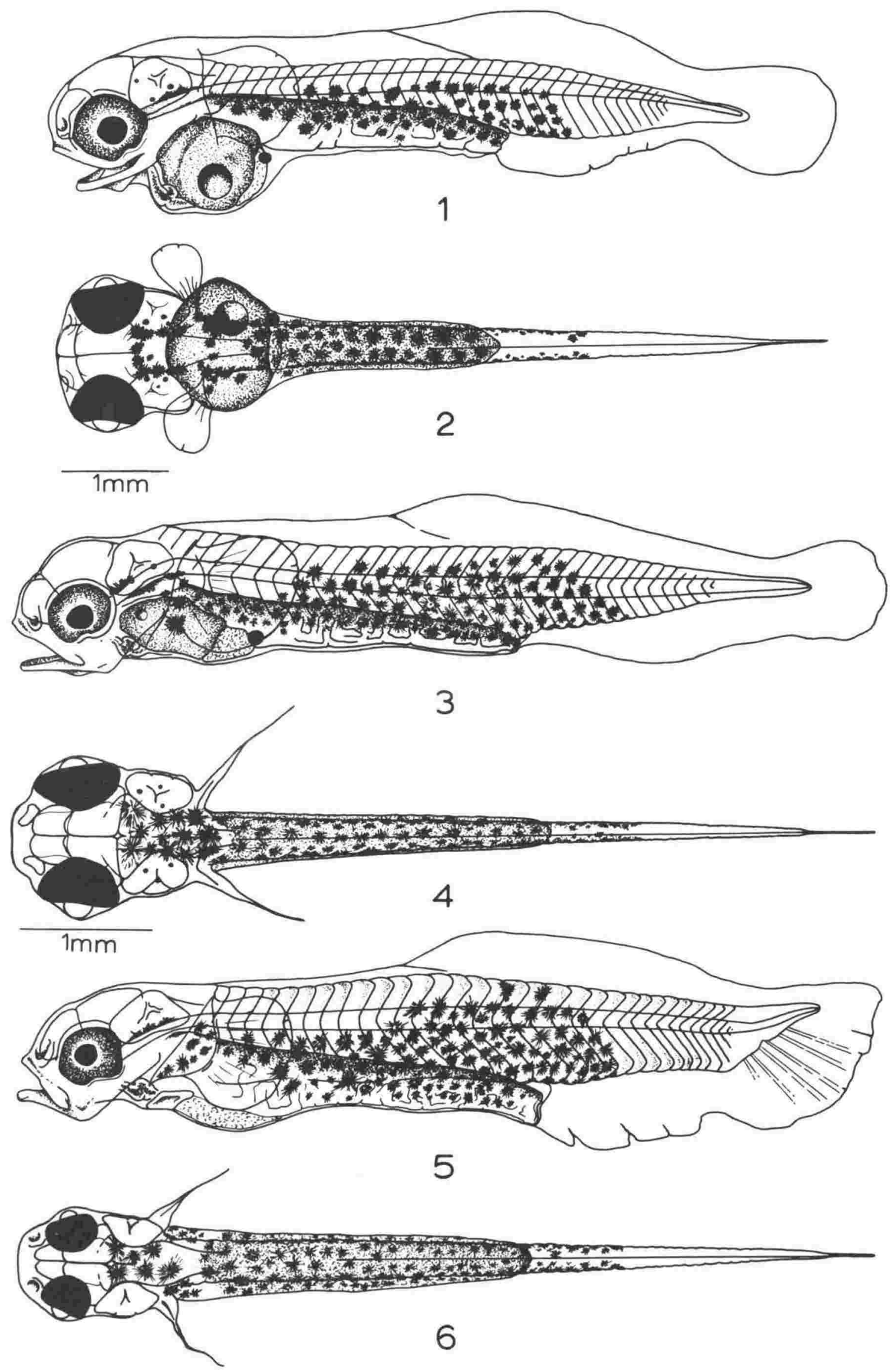
Fig. 3. Trachelochismus pinnulatus. No.1: 2nd day; 2: 3rd day; 3: 5 th day; $4: 7$ th day; 5 \& 6: 8 th day; 7 : 10th day; 8; 16 th day; $9 \& 10$ : 20 th day; 11 : 24 th day. 

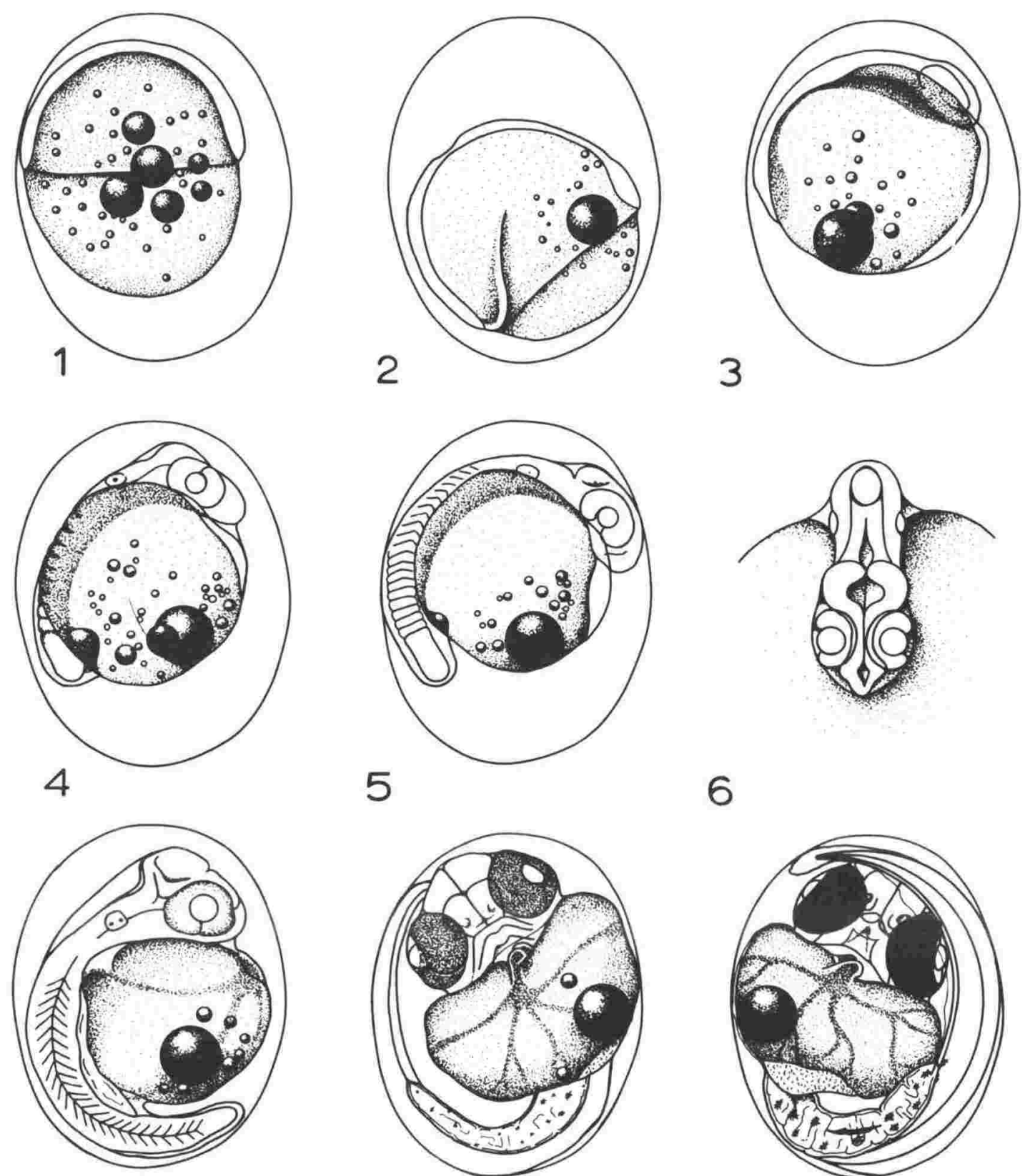

5

6

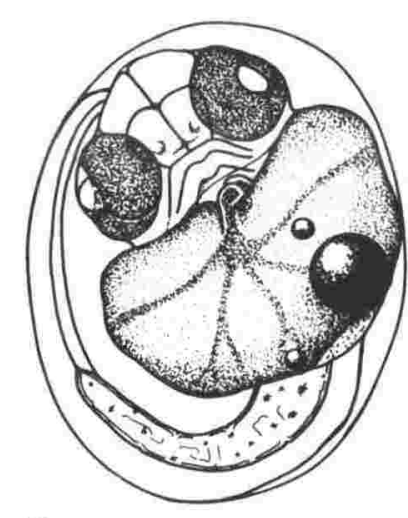

8

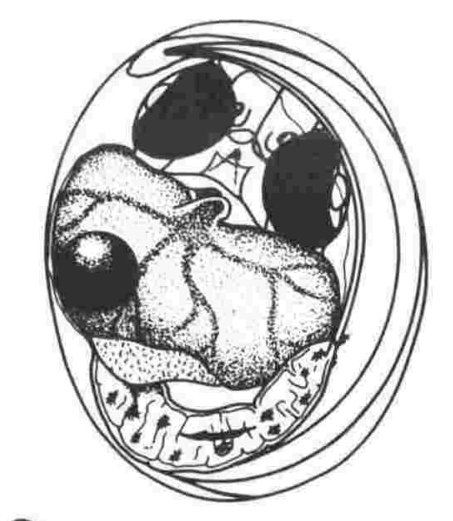

7



9

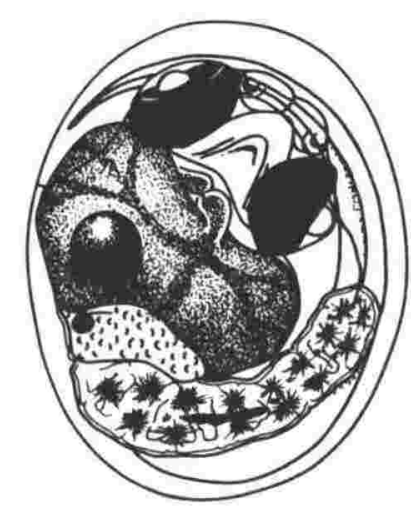

10

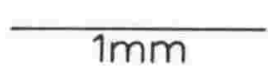


131.

Fig. 4. Trachelochismus pinnulatus. No's 1 \& 2; prolarva, $6.00 \mathrm{~mm}$ s.1. 


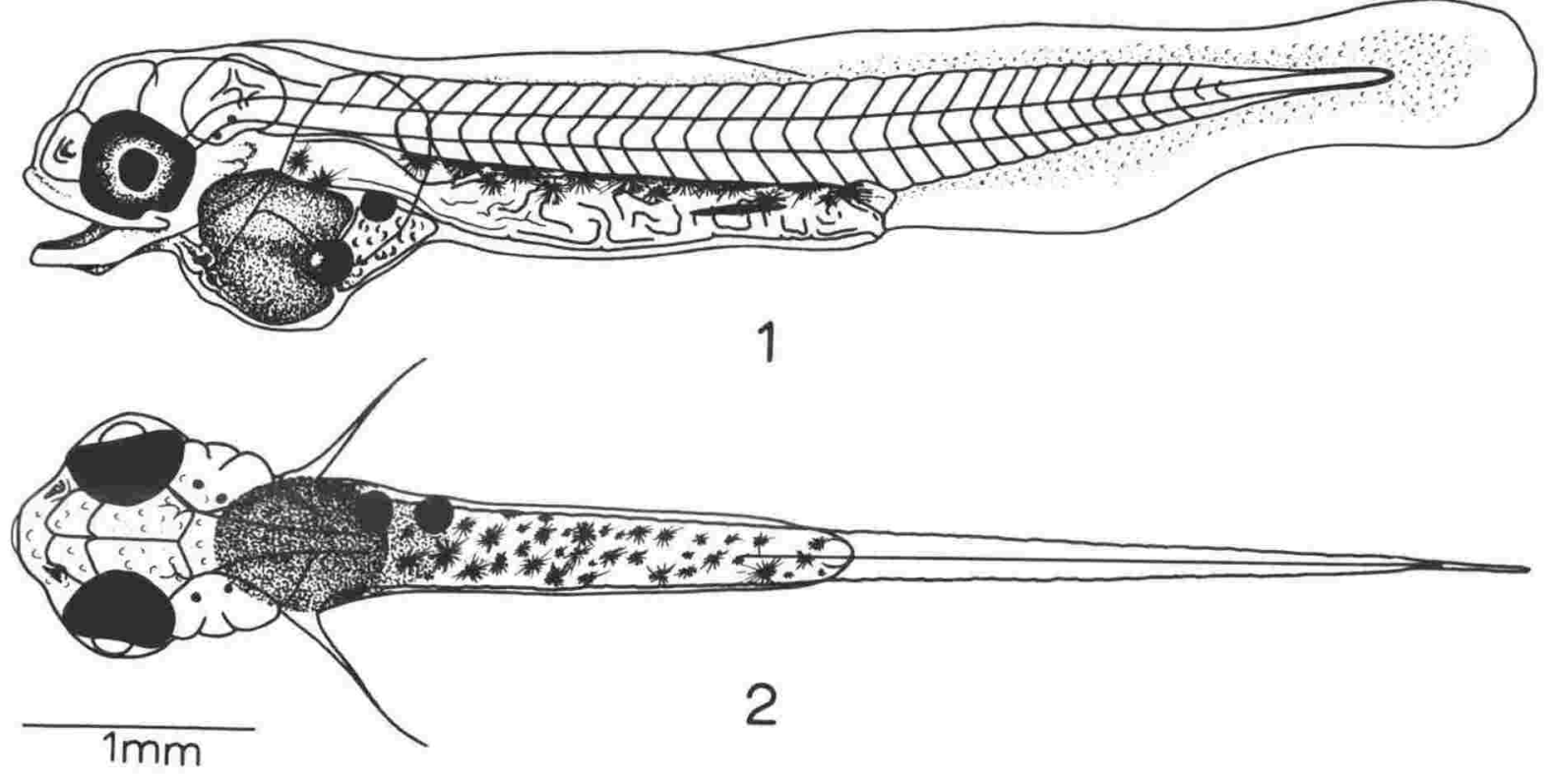


The early development of Forsterygion varium and Gilloblennius decemdigitatus (Pisces: Tripterygiidae)

By J. G. Ruck

Victoria University, Wellington

\section{Summary}

In the Wellington area $\mathrm{F}$. varium (Forster in Bloch and Schneider, 1801) spawns from early June to late November and G. decemdigitatus (Clarke, 1879) from early June to early October. Ihey deposit their eggs on firm substrates below the low tide level. These egg clusters are attended by an adult fish until hatching which under laboratory conditions occurs after 18 and 20 days respectively, in temperatures ranging from $11.5^{\circ} \mathrm{C}-13^{\circ} \mathrm{C}$. The egg development of both species is described. The prolarvae of $\mathrm{E}$. varium and G. decemdigitatus average $5.85 \mathrm{~mm}$ and $5.03 \mathrm{~mm}$ standard length respectively. Each has a long convoluted gut, and two large pigment patches in the dorsal parietal peritoneum. Prolarvae of $\mathrm{G}$. decemdigitatus have 3 large mid-dorsal melanophores interspersed by 5 smallex, much lighter, pigment cells, and 3 large, mid-ventral melanophores interspersed by 3 of the smaller cells. In contrast the prolarvae of $\underline{F}$. varium have a single row of 9-17 small, stellate melanophores along the ventral mid-line of the tail.

\section{Introduction}

Forsterygion varium (Forster in Bloch and Schneider, 1801) and Gilloblennius decemdigitatus (Clarke, 1879) are endemic tripterygiids found in shallow coastal waters throughout New Zealand. Both species typically occur in tidal pools, which even at low tide remain open to the sea.

In general F. varium (max. size $100 \mathrm{~mm}$ s.1.) is characterized by having 5 brown U-shaped markings along the dorso-lateral aspect of the body, each of which extends upwards into the dorsal fin. The brown 
areas are interspersed with yellow-green patches. The head is a mottled green and brown, while ventrally the colour is a more uniform whitishbrown. Six to 7 slightly iridescent yellow spots lie along the contour of the lateral line.

Gilloblennius decemdigitatus (max. size $90 \mathrm{~mm} \mathrm{s.l.}$ ) is a uniform black with a yellow-white ventral surface. Some individuals may be light grey speckled with brown. The anal fin is fringed with white. The head is distinctly flattened and pointed compared with other tripterygiids and the supra-orbital tentacles have approximately 10 branches each.

This paper describes the egg and prolarval development of the two species. The early development of Tripterygion capito Jenyns, 1842 and T. robustum Clarke, 1879 have been described by Ruck (1973a). Graharn (1939, 1953) briefly outlined the egg and prolarval stages and spawning behaviour of "T. varium". The present confusion in tripterygiid classification does not allow me to determine which species Grahan (1939, 1953) has described. The diameters of the eggs he gives when describing "T. varium" do not match those of Forsterygion varium (this paper) or T. robustum (Ruck, 1973a) but, although somewhat low, do approach egg dimensions for T. capito (Ruck, 1973a). Doak (1972) describes the spawning behaviour of F. variurn and Darby (1966) the spawning behaviour and prolarval stage of Helcogramma medium (Ginther, 1861).

\section{Materials and Methods}

The egg masses of $\mathrm{F}$. varium are found below low tide and are most readily obtained by diving, preferably with an aqualung. For this study eggs were scraped from the substrate into glass vials and subsequently transferred to plastic aquaria $(12 \times 1.5 \times 6 \mathrm{~cm})$ containing fresh filtered seawater. The water was changed daily and kept constantly aerated. Two plastic aquaria were used, while one contained the eggs, the other was dried and exposed to ultraviolet light. This kept bacterial numbers down, especially as the dislodged eggs rested on the bottom of the containers. In some instances it was possible to remove large portions of rock carrying eggs from the main substrate using a hammer and cold chisel. Where this was done the eggs and rock fragments were kept in the same manner 
as for Gilloblennius decemdigitatus (explained later).

On 28.6.71 8 adult $\mathrm{F}$. varium were placed in an asbestos aquarium $(250 \times 38 \times 23 \mathrm{~cm})$ at the Marine Laboratory. Several stones and shells were placed in the aquarium to provide shelter and objects on which to spawn. The tank was supplied with fresh running seawater and the fish were fed chopped fish and fresh mussels. Spawning occurred on 7.7.71 on the upper surface of a large stone. These eggs were removed and placed in a plastic aquarium (30 x $28 \times 13 \mathrm{~cm}$ ) and kept viable with a system similar to that used for $G$. decemdigitatus. The eggs laid in the laboratory confirmed the identification of those collected from the shore.

On 8.7 .70 an egg mass of $\mathrm{G}$. decemdigitatus on a portion of corrugated asbestos was collected from the Island Bay shore, and kept in a plastic aquarium. The asbestos substrate was lodged at a $45^{\circ}$ angle against the side of the aquarium with the eggs facing downwards. A fine "air-stone" was placed beneath, so that a steady stream of bubbles passed upwards and over the surface of the eggs. This method has been successful for several other species of tidal fish, namely Tripterygion capito and T. robustum (Ruck, 1973a); Trachelochismus melobesia Phillipps, 1927 (Ruck, 1971); T. pinnulatus (Forstex in Bloch and Schneider, 1801) and Diplocrepis puniceus (Richardson, 1846) (Ruck, 1973b).

This arrangement ensures a supply of oxygenated water to the eggs, while they remain in a relatively natural position away from light. The water was changed daily.

Later in the season (1970) other egg masses were collected with male $\mathrm{G}$. decemdigitatus in attendance, thus confirming the identification of the first mass collected. Each egg mass, together with the male was kept in a plastic aquarium and the aeration of the eggs was left to the male parent with some assistance from an "air-stone" in one corner of the tank.

Eggs of both species requixed for observation were easily removed from the substrate by sliding a mounted needle beneath the egg and then pipetting the dislodged egg into a petri dish. Development of the eggs of $\mathrm{F}$. varium and $\mathrm{G}$. decemdigitatus were studied and sketches of the eggs and larvae were made with a camexa lucida. An ocular micrometer was used for measuring eggs and larvae. Measurements of the prolarvae were based on those recommended by Hubbs and Lagler (1958), with the exception of head length, taken here as the distance from the tip of the snout to the 
back of the otic capsule. This is more definite than the opercular flap which is often not visible. The bulk of the egg and larval terminology is based on that used by Tavolga and Rugh (1947), Rugh (1948), Balinsky (1965) and New (1966).

\section{Spawning and development of Forsterygion varium}

Along the coast around Wellington Forsterygion varium spawns from early June to late November. Egg masses are found below low tide on exposed vertical surfaces of large stones or on overhanging portions of underwater cliffs. No egg masses were found beneath stones unlike those of $\mathrm{G}$. decemdigitatus. Eggs are laid in a single sheet on top of encrusting algae and tube worms. The egg masses vary in size from $2-3 \mathrm{~cm}$ in diametex, containing $1-2$ hundred eggs to about $11 \mathrm{~cm}$ in diameter and containing several thousand eggs.

The large clusters characteristically have subgroups of eggs at different stages of development, indicating successive and different spawnings on the same substrate. It is not clear whether different females are responsible for the total number of eggs in each mass. The presence of eggs at different stages of development in any one mass is not uncommon for intertidal fish. I have found mixed batches of eggs for Tripterygion capito, T. robustum (Ruck, 1973a) Gilloblennius tripennis (Forster in Bloch and Schneider, 1801) Trachelochismus melobesia (Ruck, 1971) T. pinnulatus and Diplocrepis puniceus (Ruck, 1973b). Darby (1966), records a similar situation for Helcogramma medium. This spawning behaviour is not restricted to New Zealand fish as similar conditions have been recorded with the clingfish Gobiesox strumosus Cope, 1870 (Runyan, 1961) and the sponge blenny paraclinus marmoratus (Steindachner, 1866) (Breder, 1941) in which the male "guarding" the nest may mate with different passing females.

Spawning of $\mathrm{F}$. varium was observed on 7.7 .71 in the large asbestos aquarium. The eggs were laid on the upper surface of an irregularly shaped stone. Courtship behaviour was not seen. The spawning behaviour was the same as that described by Doak (1972). During spawning, however, several other members of the same species attempted to swallow the freshly laid eggs, but were persistently opposed by the mating male. However, 
this behaviour was somehow transposed to the male who also began to eat the new eggs. Because of this the egg mass was removed from the tank and placed in another aerated aquarium. This aberrent behaviour may have been induced by overcrowding or the artificial nature of the spawning area.

Somewhat related behaviour was noted in the field, when the eggs were scraped from the main masses. Loose eggs were rapidly consumed by the attendant male. A similar event is described by Weisel (1947) where for no obvious reason fresh laboratory-spawned eggs of the mudsucker Gillichthys mirabilis Cooper, 1863, were eaten by the parents. The depositing of eggs on exposed surfaces is relatively uncommon for New Zealand li.ttoral fish. In most cases eggs are laid beneath rocks or in crevices e.g. previously mentioned New Zealand species as well as the rockfish Acanthoclinus quadridactylus (Forster in Bloch and Schneider, 1801) (Jillet 1968). Because the eggs of F. varium are lajd on vertical exposed surfaces no parental aeration appears required. In the above mentioned species the eggs lie beneath objects and therefore require certain physical activity on the part of the attendant parent to ensure a flow of oxygenated water over them. Nevertheless the male F. varium, which is generally darker than the female during breeding, remains close to the egg mass and actively repels fish or human intruders.

The protective activities of littoral fish particularly blennioid species have been noted, for Blennius gattorugine L. (Brown, 1929), Petroscixtes trossulus (Jordon and snyder, 1902) (Hinokiyama in Gibson, 1969), Petroscirtes bipunctatus Day, 1875 (Dutt and Rao, 1960), Salarias enosimae (Jordon and Snyder, 1902) (Mito, 1954), Blennius pholis L. (Qasim, 1956), Acanthoclinus quadridactylus (Forster in Bloch and Schneider, 1801) (Jillet, 1968) as well as the previously referred to New Zealand blennioid and gobiesocid species that I have observed. Breder (1941) describes the elaborate and somewhat vigorous procedure that the male sponge blenny Paraclinus maxmoratus undergoes to oxygenate its eggs. 


\section{Development of the Egg}

The eggs are moxe or less spherical and uniformly covered with fine elastic filaments which converge to form tendrils. These radiate from small points on the chorion $0.10 \mathrm{~mm}$ apart. The lower tendrils entwine with projections fxom the substrate and with the tendrils of adjacent cells (Fig.1, no's 1 \& 2). When first laid the chorion is soft and translucent white and the tendrils seem to adhere to the substrate immediately.

After water hardening and consequent expansion of the chorion (1 hour after fertilization) the lower tendrils are drawn tight thus anchoring the egg firmly. This is important considering the degree of exposure of these eggs to turbulence. The tendrils of newly laid eggs are white and impart a fluffy cotton wool appearance to the whole egg mass. However, due to exposure and the adhesive nature of the tendrils fine silt and detritus accumulates on the eggs thus giving a brown colour to more advanced masses of eggs. The numerous adhesive filaments obscure the limits of the chorion making accurate measurements very difficult. It is possible to clear parts of the chorion of inaividual eggs by removing them from the masses with tweezers and gently brushing ther over the surface of a dry cloth. The diameter of the chorion ranges from 1.12-1.23 mm, identical to that of I. robustum (Ruck, 1973a). The yolk is large (about $1.1 \mathrm{~mm}$ in dia.), spherical and transparent to pale yellow in colour. Within the yolk there is a cluster of pale yellow oil. globules that vary from egg to egg, in number (15-40) and size (microscopic to $0.15 \mathrm{~mm}$ ). Viewed in the masses, these oil globules early in development appear bright orange. Development of the eggs to hatching (Fig.1, no's 1-12), took a minimum time of 18 days at a temperature of about $12^{\circ} \mathrm{C}$.

One hour (Fig.1, no.1). There is a gradual accumulation of protoplasm at one end of the yolk forming a transparent slightly raised zone. The yolk surface beneath this first "cell" is deeply ridged.

Three hours. First cleavage takes place at right angles to the yolk surface.

Seven-eight hours (Fig.1, no.2). A second cleavage occurs at right angles to the first producing 4 rounded cells or blastomeres. 
Nine-ten hours (Fig.1, no.2a). The eight cell stage is attained by the equal cleavage of each of the 4 blastomeres giving two rows of 4 cells.

Eighteen hours (Fig.1, no.3). Repeated cleavages at right angles and horizontal to the yolk surface produce a pronounced mass of cells. Individual cells can still be seen and the exposed surface of each is rounded. No blastocoel is visible. At the periphery of the cellular mass the cells are open to the periblast (the nucleated membrane surrounding the yolk). This is the morula stage.

Fortytwo hours (Fig.1, no.4). The blastula stage is reached and consists of a rounded cap of tiny individually indistinct cells (blastoderm) overlying a shallow blastocoel. There is considerable confusion in the literature in the use of the terms blastodisc and blastoderm. Battle (1940, 1944) applies the term blastodisc to the very early "one-cell" stage through to the point where the blastodisc begins to spread around the yolk. At this point she refers to it as the spreading blastoderm. Oppenheimer (1937) also considers the "one-cell" stage to be the early blastodisc but in contrast to Battle applies the term blastoderm as early as the four-cell stage, retaining it through to the closing of the blastopore. New (1966) using information fxom work by Oppenheimer (1937) and Armstrong and Child (1965), avoids "blastodisc", but uses blastoderm to denote the mass of cells found just prior to blastocoel formation. Like oppenheimer (1937) he applies the term blastoderm to cellular tissue up to the closing of the blastopore. Balinsky (1965) refrains from using blastodisc or blastoderm until the blastula stage is reached, at which point he calls the upper cells of the blastula, overlying the blastocoel, the blastoderm, and because they form a sort of disc the whole structure a blastodisc. His use of the term blastoderm as late in development as this, relates to the fact that only at this point do the cells overlying the blastocoel adhere closely enough to one another to form a type of epithelial mass (hence blastoderm). Balinsky (1965) continues to use blastodisc when referring to the cellular mass encircling the yolk right through to blastopore closure. In a sense blastodisc and blastoderm are synonomous as Bodemer (1968) considers them to be. In this study and others I have used the terms as laid down by Balinsky (1965). 
Third day (Fig.1, no.5). The blastodisc covers over half of the yolk surface. The rim of the disc is thickened by epiboly to form the gerrn ring. On one side of the blastodisc where involution is the greatest the embryonic shield develops. The thin sheet of cells of the disc other than the embryonic shield and germ ring is the extra-embryonic ectoderm that eventually encloses the yolk as the yolk-sac. The lip of the blastodisc is preceded by a thin sheet of cells, as it migrates around the yolk.

Early fourth day (Fig.1, no.6). The blastodisc covers virtually all the yolk and the blastopore is very small. The embryonic axis (including the notochord, neural keel and somites) is well defined. of these the neural keel is the most obvious encircling half the yolk and being expanded considerably in the region of the primordial brain. The primordial brain is embedded slightly in the yolk, is irregular in outline and expanded laterally due to the formation of the optic vesicles as outgrowths of the prosencephalon. A broad and shallow perj.cardial cavity lies forward of the head.

Fourth-fifth day (Fig.1, no.7). The blastopore is closed and Kupffer's vesicle is present beneath the well defined tail bud. The head of the embryo is distinct and embedded slightly in the yolk. The optic vesicles are completely separated from the prosencephalon. Lateral to each vesicle there is a thickening of the ectodermal tissue for the lenses formation. The outside face of each optic vesicle is scalloped with the onset of the optic cup development. Half-way along the embryo there are faint outlines of myomere block rudiments lateral to the neural keel. The pericardial cavity has expanded beneath the head slightly. Seventh day (Fig.1, no.8). The embryo encircles more than half of the yolk circumference but the tail bud is still attached. The heart beats very faintly well beneath the head. There is no blood flow. By extending back under the head the pericardial cavity lifts the anterior part of the head from the yolk. The main lobes of the brain are clearly defined, and the ventricles are visible especially in the optic lobes and the medulla. The cerebellum (metencephalon) is represented by the rhombic lip on the anterior border of the fourth ventricle. The auditory placodes (pits) are present lateral to the medulla as shallow depressions in the ectoderm. The lenses of the eyes are quite spherical and fill the entire optic cup. The chorioid 
fissure is wide. The myomeres extend well into the tail, the anterior most ones are chevron shaped. Fifteen to 20 stellate melanophores lie scattered over the yolk surface under the yolk sac. A small and diffuse cluster of melanophores lie in front of each eye and a slightly larger but similax cluster behind.

Four other pigment clusters are present, one posterior to each auditory placode and two fainter ones half way along the embryo. There are 4 elongate melanophores in the tail bud. The oil globules within the polk lie directly beneath the head.

Ninth day (Fig.1, no.9). The heart beats regularly sending blood rapidly through the vessels of the body but more slowly through the wider vitelline vessels. The heart itself is little more than a contractile tube with virtually no diffexentiation into chambers. The most anterior portion of the tube (sinus venosus) extends slightly forward of the head. The optic lobes have expanded dorsally and laterally pushing the eyes sideways, and there is a deep cleft in the myelencephalon. Each ventricle is covered by a thin roof that later thickens. The eyes have increased in size so that each lens no longer occupies the whole of the optic cup. Approximately 18 oil globules of varying sjzes remain in the yolk. The main areas of pigmentation in the embryo have increased in size due to an increase in the number of melanophores. The melanophores within each group blend with each other making accurate counting impossible. The chorioid of the eyes are faintly pigmented, and the otic vesicles are triangular and contain two visible otoliths. The tubular gut is visible extending back from beneath the otic vesicles, and possessing several melanophores distributed around its perimeter. The tail is well clear of the yolk sac curving about the yolk peximetex and finishing close to the head. Two rows, each with 5-6 melanophores run along the mid-ventral aspect of the tail. Three more lie in the dorsal tip of the tail. Twelve to 15 stellate melanophores are scattered over the yolk surface. The posterior most myomeres are still block shaped, without the characteristic chevron shape of the anterior ones. The embryo undergoes infrequent contraction and relaxation of the trunk and tail myomeres. The notochord is prominent running from beneath the medulla to the tip of the tail, appearing as a transparent strap in the mid-line of the embryo. 
Eleventh day (Fig.1, no.10). All the chambers of the heart are differentiated. The lateral vitelline vessels (future ductus cuvieri) emerge from the embryo anterior to the pectoral bud and traverse the yolk to enter the sinus venosus. A third vitelline vessel (future left post cardinal vein) emerges from beneath the gut posterior to the developing liver (left side) to pass around the ventral yolk surface to enter the sinus venosus at a point between the entry of the two lateral vessels. The brain lobes are rounded and the cleft in the medulla narrower. The cerebellum is obscrued by the rearward projection of the optic lobes. Chorioid pigmentation is dark but the lenses are still visible with transmitted light. Each otic vesicle is "bean-shaped" having lost the earlier triangular outlinè. The pectoral primordia are present as thick lobes of tissue behind each otic vesicle. Total pigmentation has altered little, except that individual pigment cells are in general larger, and the number of yolk melanophores is reduced to 6 or 7. The melanophores along the ventral mid-line of the tail have combined to form a single row of about 7 pigment spots. Very few oil globules remain in the yolk, of these the largest does not exceed $0.2 \mathrm{~mm}$ in diameter. The dorsal, ventral and caudal fin folds show signs of growth. The embryo contracts and relaxes frequently resulting in considerable rotation within the chorion.

Fourteenth day (Fig.1, no.11). The anterior most portion of the head is free from the pericardial membrane. The head has expanded dorsally and laterally drawing the otic vesicles closer to the eyes. Rotation of the embryo is infrequent due to increased growth of the embryo resulting in cramped conditions inside the chorion. The tail overlaps the head considerably and the upper and lower fin folds are quite broad; the lower being granular in appearance. The chorioid pigmentation is almost complete. As a result certain melanophores behind the eyes are obscured and are visible from the dorsal aspect only (Fig.l, no.lla). The number of melanophores behind each otic vesicle is reduced to about 4. The group of 7-8 small melanophores in the dorsal parietal peritoneum is very closely aggregated, and lies in the position of the laxge pigment patch found above the gut in the prolarval stage. One large pigment patch lies antero-dorsally to the anus in the parietal. peritoneurn, and sends long dark processes about the hind gut. The urinary vesicle is visible just behind the anus. 
Eighteenth day (Fig. 1, no.12). The embryo is very tightly coiled within the egg. The chambers of the heart are exposed due to the lifting of the head from the yolk surface. This is also in part due to the considerable reduction in yolk. As this stage is just prior to hatching the embryo is identical in all respects to the newly hatched prolarva (Fig.2, no's 1 and 2). During the later stages of embryological development the embryo remains quiet. Just before hatching, however, the embryo becomes agitated and gyrates wildly, at the same time flexing the tail as if to exert pressure on the chorion. This activity appears to coincide with a general weakening of the chorion. In fact the chorion goes through stages of varying strength. Soon after fertilization the chorion is quite delicate being very easily ruptured by a needle probe. This initial weakness is followed by a long period of relative strength where a great deal of effort is required to penetrate the chorion with a needle. The bulk of embryological growth takes place during this period. The final weakening of the chorion at hatching is possibly due to the liberation of enzymes by the embryo. Fundulus heteroclitus (Gmelin, 1766) (Armstrong, 1936) releases an enzyme from cells of the mouth and gill cavities that serves to weaken the chorion at hatching. Armstrong (1936) found that this weakening of the chorion had to be coupled with general agitation of the embryo before hatching could take place.

The chorion is eventually ruptured (F. varium) and the prolarva is released.

Prolarva (Fig.2, no's 1 \& 2). Prolarvae on hatching swim immediately to the water surface and then migrate to the region of greatest light intensity. Prolarval length on hatching xanges from $5.35-6.35 \mathrm{~mm}$ standard length. The amount of yolk present varies. The yolk with 1-2 small oil globules is bordered anteriorly by the heart and posterioriy by the liver and gall-bladder. The gut is long and convoluted with two large pigment patches on the dorsal parietal peritoneum, one anterjor to the anus and the other above the liver. The ventral fin fold is interrupted at the anus, giving a small fold beneath the gut and a larger one running the full length of the tail. The ventral fin fold is constricted beneath the tip of the tail, then expands to form a spatulate caudal fold which in turn extends to the dorsal region to form the continuous dorsal fin fold. The 
anterior most portion of the dorsal fin fold has its origin directly above the cleithrum. Along the ventral mid-line of the tail are 9-17 stellate melanophores that may blend together to form a diffuse black line. No dorsal mid-line pigments are present. The notochord is broad and segmented. The lobes of the brain are pronounced, and the otic vesicles are rounded and close to the eyes that are fully pigmented. An oblique refractive line forward of the pectoral fin outlines the cleithrum. From $1-2$ individual melanophores lie in the epithelial tissue lateral to the yolk. The basic pattern of prolarval blood flow is as follows. Blood leaves the ventricle and passes directly to the gills that are tucked in behind the eyes. From the gills it passes backward to the dorsal aorta via paired lateral aortae (visible in dorsal view only). The dorsal aorta carries blood back along the body, directly beneath the notochord, and gives off tiny vessels to the gut and myotomes. Some of these vessels are so small as to allow the exythrocytes to travel in single file only. The dorsal aorta leads directly into the caudal artery, above the anus, that continues to the tip of the tail where it loops to form the caudal vein that carries the blood forward again toward the head. The caudal vein also receives blood from tiny vessels draining the myotomes. Once reaching the anus the caudal vein gives off many tiny vessels that pass around the rectur to join with a vein ventral to the gut. The caudal vein continues a little forward of the anus and branches sending a prominent vessel down the side of the rectum (rectal arch) to join beneath (as the left posterior cardinal vein) and continues on under the liver and yolk to enter the sinus venosus. The gut is extensively drained by capillaries that converge along the ventral gut to pass forward into the liver as the hepatic portal vein. Erythrocytes are clearly visible percolating through the hepatic sinusoids. The hepatic vein travels from the liver across the yolk into the sinus venosus. This hepatic system is visible on the left side only. A further vein (right posterior cardinal vein) carrying blood forward arises from the top of the rectal arch and passes beneath the dorsal aorta to enter the right ductus cuvieri. Blood supply to the head is obscured by the eyes. A series of vessels travels beneath the bxain from the gills carrying blood to the anterior head. From these vessels fine capillaries traverse the brain and collect in the left and right anterior cardinal veins that carry the blood back to the ductus cuviexi. The ductus cuvieri pass vertically down grooves in the sides of the yolk sac to the sinus venosus. 
The average measurements of 2.5 prolarvae are as follows:

$\begin{array}{lc}\text { Standard length (mm) } & 5.85 \\ \text { Total length (mm) } & 6.10 \\ \text { Head length (mm) } & 0.90 \\ \text { Eye length (mm) } & 0.39 \\ \text { Snout to anus (mm) } & 2.37 \\ \text { Mid-ventral melanophores } & 15(9-17) \\ \text { Lateral yolk melanophores } & (1-2)\end{array}$

Spawning and development of Gilloblennius decendigitatus

In the Island Bay area near Wellington G. decemdigitatus spawns from early June to early october. Egg masses are found below low tide in areas of dense algal growth. The undersides of flat stones seem preferred although egg masses may also be found on coralline encrusted stones and various synthetic artifacts such as clay pipes, tin cans and asbestos sheets. The flat irregular shaped masses vary in size and number of eggs similar to that of Forsterygion varium.

One less characteristic egg mass, however, was found spread thinly over an area $3 \times 20 \mathrm{~cm}$. The large clusters characteristically have subgroups of eggs at different stages of development, again indicating different spawning times on the same substrate. There is as much as 5 days difference in development time between some eggs. The most advanced eggs are usually found in the middle of the egg masses representing the site of the earliest spawning on the substrate. The eggs are essentially transparent, although the presence of numerous tiny white dots on the chorion of each egg makes the masses quite conspicuous. Virtual.1y all. the egg masses observed on the shore had an adult G. decemdigitatus somewhere close by.

\section{Development of the Egg}

The egg is spherical with a mean chorion diameter of $1.17 \mathrm{~mm}$ and ranging from $1.12 \mathrm{~mm}-1.20 \mathrm{~mm}$. Some eggs may be dorso-ventrally depressed with depths of only $0.8 \mathrm{~mm}$. At the blastula stage the yolk is essentially 
spherical, colourless, averages $0.90 \mathrm{~mm}$ and contains 10-11 oil globules associated with 3-4 pigment spheres. Attached at various points over the entire chorion are blobs of matted tendrils, that are not as attentuated as those of Forsterygion varium. These serve to fasten the eggs to the substrate and to each other. All eggs removed from the shore were at the blastula stage, and these were estimated to be between 30-40 hours old. Development to hatching (Fig.3, no's 1-9 and Fig. 4, no's 10-12) took 22 days at a temperature ranging from $11.5^{\circ} \mathrm{C}-13^{\circ} \mathrm{C}$.

Thirty-forty hours (Fig.3, no.1). A prominent blastoderm lies at one end of the yolk, overlying a shallow blastocoel. There is a thin skixt of cells spreading outward from the base of the blastodisc. This is the blastula stage. The oil globules and pigrnent spheres aggregate in the dorsal most part of the yolk, depending on the attitude of the egg at the time of removal from the substrate.

Third day (Fig.3, no.2). The blastodisc covers nearly half of the yolk. The faint outline of the embryonic shield and germ ring indicate that epiboly is well under way. Some of the oil. globules have coalesced resulting in 6-8 large ones plus 10-12 small oil droplets. The pigment spheres have also coalesced to form a single dark mass.

Sixth day (Fig.3, no.3). The embryo is well defined and is raised above the surface of the yolk right back to the prominent tail bud. The optic vesicles which axe completely separated from the prosencephalon, are indented slightly on the lateral faces to receive the developing lenses. Approximately 15 pairs of myomeres are present lateral to the neural keel. The pigment has migrated to the yolk surface where it is arranged in cells as irregular curved patterns. No distinct melanophores are visible at this stage. The yolk measures $0.80 \times 0.60 \mathrm{~mm}$.

Seventh day (Fig.3, no.4). The walls of the brain axe infolded defining the third and fourth ventricles and the main lobes. A slit like depression between the second and third myomere marks the auditory placode. The lenses are well rounded filling the optic cup and the chorioid fissure is closed. A smudge of brown pigment is visible behind each eye and each auditory placode. The melanophores on the yolk surface are now individually defined and arranged in two closely aligned rows that form S-shaped and semi-circular patterns. The overall shape, size and number of melanophore groups varies slightly with different embryos although 
the pattern is generally similar either side of a single yolk. Four large and several small oil globules remain in the yolk, the largest measuring no bigger than $0.20 \mathrm{~mm}$ in diameter. The myomere blocks have developed to the end of the tail, which is just free of the yolk.

Eighth day (Fig.3, no.5). The heart beats slowly and feebly with the extremely faint blood restricted to an ebb and flow within the heart itself. The heart lies deep in the pericardial cavity beneath the anteriox part of the head. Extra pigmentation to that already mentioned includes one melanophore in front of each eye and an obscure series of melanophores along the ventral mid-line of the tail, beginning at the point where the tail lifts clear of the yolk. The anterior myomeres are chevron shaped while those in the tail are still rounded and oblong. The gut is long and obscured by being embedded in the yolk. The vent is visible just in front of the tail melanophores. The whole embryo flexes periodically.

Tenth day (Fig.3, no.6). The heart beats regularly and strongly, and blood flows through the major vessels. The pericardial cavity is expanded forward of the head. A faint haze of pigmentation covers the chorioid of the eye, and the lenses no longer fill the optic cups. The tail arches over the yolk to a point just in front of the snout. The mid-ventral tail melanophores have increased although exact counting is impossible due to the overlapping of adjacent pigment cells. Six to 7 stellate melanophores lie on the lateral aspect of the free tail. The otic vesicle is triangular in outline.

Thirteenth day (Fig.3, no.7). Individual pigment cells on the yolk are beginning to lose definition by considerable spreading and intexdigitating with adjacent cells. The lobes of the brain are well defined and rounded and the chorioid pigmentation is quite dark. The tail follows the curvature of the chorion and overlaps the head slightly. The embryo still has considerable room at this stage and is able to twist and turn frequently. Intense light appears to accentuate this movement. Seven melanophores are present along the ventral mid-line of the tail, while 9 are visible along the dorsal mid-line. The trunk of the embryo is essentially free of pigmentation. The yolk encloses a single oil globule about $0.30 \mathrm{~mm}$ in diameter. 
Fourteenth day (Fig.3, no.8). The head has increased in depth and the anterior part is lifted from the yolk surface. The heart lies within the pericardial cavity forvard of the head. The melanophores on the yolk form two main diffuse sheets that fan out on either side of the embryo, from just in front of the pectoral buds. Individual melanophores are extended and 'spidery' with appendages extensively overlapping and interlocking with those of adjacent pigment cells. Pigment clusters behind the eyes and otic capsules are unchanged. Several dark pigment cells have gathered about the gut, which now extends clear of the yolk surface. The melanophores along the dorsal and ventral mid-lines of the tail present themselves as an unbroken series of intertwined cells, making counting of individual cells impossible. The dorsal and ventral fin folds are faintly visible.

Fifteenth day (Fig.4, no.1). The yolk is reduced being quite dense and somewhat granular in appearance. The broad sheets of melanophores have dissociated leaving only a few large 'spidery' pigment cells on the surface of the yolk. The others seem to have become incorporated into the embryo particularly above the gut. The eyes are darkly pigmented but the lenses are still visible through the chorioid with transmitted light. The tail has lengthened, overlapping the head and extending back along the body as far as the pectoral buds.

Seventeenth day. Two large pigment patches are situated in the parietal dorsal peritoneum, one forward of the anus and the other above the yolk sac.

Twentieth day (Fig.4, no.2). The embryo lies tightly coiled within the chorion, restricting movement to an occasional roll. The yol.k superficial pigmentation is reduced to 3-4 small melanophores. A small melanophore has appeared in the epithelium at the back of the yolk sac. The melanophores in the tail ( 3 ventral and 3 dorsal) are now individually distinct. One small stellate melanophore is present in front of each eye, directly behind the shallow olfactory pits. Pigmentation of the eyes appears complete. The snout is away from the yolk far enough to expose the 3 chambers of the heart and the outline of the developing lower jaw.

Prior to hatching which occurs on about the twenty second day, the embryo twists more violently than usual, until the weakened chorion is ruptured. The tail eraerges first, leaving the much larger yolk and 
head within the chorion. After a rest the embryo resumes its struggle to finally release itself.

Prolarva (Fig.4, no's $3 \& 4$ ). As is the case for many intertidal species mass hatching can be induced by disturbance of the egg masses, particularly by the addition of fresh cool water to the aquarium. It is possible that this condition has survival value in that hatching may well be induced by a rising tide that brings fresh cool water to the egg masses, on the shore. It would seem desirable for hatching to occur on a rising tide as this would prevent prolarvae being stranded in pools for any great length of time, where they may easily be preyed upon by tidal fish. The eggs of Galaxias attenuatus (Jenyns, 1842) which essentially incubate out of water, are induced to hatch only when immersed by a spring tide (Benzie, 1.968; McDowall, 1968). Prolarvae on hatching also swim very rapidly to the surface of the water and toward the strongest light intensity. Again it is possible these adaptations carry the prolarvae quickly from the dark side of stones to areas furthest away from possible predation.

The yolk is typically very reduced and is bordered anteriorly by the heart and posteriorly by the liver and gallbladder. A single small oil globule is present within the yolk. The lower jaw articulates freely and quivers at regular intervals. For a short time after hatching 3-4 tiny melanophores persist posterior to each otic capsule, but soon fade and disappear. The gut is long, tubular and convoluted and has the two large pigment spots in the dorsal parietal peritoneum. A third pigment patch lies at the apex of the liver. The most characteristic arrangement of melanophores is along the dorsal and ventral mid-lines of the tail. Dorsally there are 3 large melanophores interspersed by 5 smaller, much lighter (brown) pigment cells. Similarly there are 3 large melanophores along the ventral mid-line interspersed by 3 lighter pigment cells. One more very faint melanophore is sometimes present at the very end of the tail.

The measurements of 25 prolarvae are as follows:

$\begin{array}{ll}\text { Standard length (mm) } & 5.03 \\ \text { Total length (mm) } & 5.24 \\ \text { Head length (mm) } & 0.81 \\ \text { Eye length (mm) } & 0.40 \\ \text { Snout to anus (mra) } & 1.86 \\ \text { Total mid-dorsal melanophores } & 8(6-9) \\ \text { Total mid-ventral melanophores } & 3(3-5)\end{array}$


Five-day Prolarva (Fig.4, no's $5 \& 6$ ) (5.8 mm s.1.).

The yolk sac is virtually completely absorbed. The pigment spots above the gut are closer together. All the brown pigment cells along the dorsal and ventral mid-lines have disappeared, leaving 3 large melanophores ventrally and dorsally. A new stellate melanophore is present in the integument above the optic lobes. The head is narrower than the prolarval stage and the snout is longer.

\section{Acknowledgement}

I would like to thank Dr P.H.J. Castle, Department of Zoology, Victoria University of Wellington, for his constructive criticism of this paper.

\section{$\underline{\text { References }}$}

Armstrong, P. B. 1936.

Mechanism of hatching in Fundulus heteroclitus. Biol. Bull. max. biol. Lab., Woods Hole, 71 (2): 407.

Armstrong, P. B. and Child, J. S. 1965. Stages in the normal development of Fundulus heteroclitus. Biol. Bull. mar. biol. Iab., Woods Hole, 128: 143-168.

Balinsky, B. I. 1965.

An introduction to embryology, Philadelphia and London:

W. B. Saunders Co., 673 pp., 443 fig., 23 tab.

Battle, H. I. 1940.

The embryology and larval development of the goldfish (Carassius auratus L.) from lake Erie. Ohio Jour. Sci., 40(2): 82-93, 21 fig., 1 tab., 1 graph.

Battle, H. I. 1944 .

The embryology of the Atlantic salmon (Salmo salar Linnaeus).

Can. J. Res., 22 (5) : 105-125, 39 fig., 4 tab. 
Benzie, V. L. 1968.

Some ecological aspects of the spawning behaviour and early development of the common whitebait, Galaxias maculatus attenuatus (Jenyns). Proc. N.z. ecol. Soc. 15: 31-39, 13 fig., 1 tab. Bodemer, C. W. 1968.

Modern embryology. New York: Holt, Rinehart and Winston, Inc., 475 pp., 20. 7 fig.

Bredex, C. M. 1941.

On the reproductive behaviour of the sponge blenny Paraclinus marmoratus (Steindachner). Zoologica, N.Y., 26 (22): 233-236, 6 fig.

Brown, E. M. 1929.

Notes on the Breeding Habits and early larvae of the Tompot

blenny. Proc. Zool. Soc. Lond., 1929, 487-489, I fig.

Darby, M. M. 1966.

The ecology of fishes in tidal rock pools with a revision of the common littoral species Tripterýgion nigripenne cuvier and Valenciennes. Unpublished M.Sc. Thesis, University of Canterbury. Doak, W. 1972.

Fishes of the New Zealand region. Auckland: Hodder and stoughton, 132 pp., 48 fig., $48 \mathrm{pl}$.

Dutt, S. and Rao, V. V. 1960.

On the breeding habits and early development stages of

Petroscirtes bipunctatus Day. J. Zool. Soc. India, 12 (2): 158-161, 1 fig.

Gibson, R. N. 1969.

The biology and behaviour of littoral fish. Oceanogr. Mar. Biol. Ann. Rev., 7: 367-410, 5 fig., 3 tab.

Graham, D. H. 1939.

Breeding habits of the fishes of Otago Haxbour and adjacent seas. Trans. Proc. R. Soc. N.z. 69: 361-372, 7 pl.

Graham, D. H. 1953.

Treasury of New Zealand Fishes. Wellington: A. H. \& A. W. Reed. 404 pp., 153 fig.

Hubbs, C. L. and Lagler, K. F. 1958.

Fishes of the great lakes region. Michigan: Cranbrook Institute of Science. 213 pp., 251 fig., 6 tab. 
Jillett, J. B. 1968.

The biology of Acanthoclinus quadridactylus (Bloch and Schneider) (Teleostei-Blennioidea). II Breeding and development. Aust. J. mar. Freshwat. Res., 19: 9-18, 5 fig., 2 tab.

McDowall, R. M. 1968.

Galaxias maculatus (Jenyns), the New Zealand whitebait. Fish. Res. Bull., (2) : 83 pp., 43 fig., 8 tab.

Mito, S. 1954.

Breeding habits of a blennioid fish, Salarias enosimae

Jap. J. Ichthy., 6: 144-152, 4 fig., 3 tab.

New, D.A.T. 1966.

The culture of vertebrate embryos. London: Logos Press, Academic Press, 245 pp., 46 fig., 3 tab., 16 pl.

Oppenheimer, J. M. 1937.

The normal stages of Fundulus heteroclitus. Anat. Rec., 68 (1): $1-15,33$ fig., 3 pl.

Qasim, S. Z. 1956.

The spawning habits and embryonic development of the shanny (Blennius pholis L.). Proc. zool. Soc. Lond., 127: 79-93, 5 fig., 5 tab., 1 pl.

Ruck, J. G. 1971.

Development of the lumpfish Trachelochismus melobesia (Pisces:

Gobjesocidae) Zool. Publs Vict. Univ. Wellington, 57: 1-9, 4 fig.

Ruck, J. G. 1973a.

Development of Tripterygion capito and T. robustum (Pisces:

Tripterygiidae). In press.

Ruck, J. G. $1973 \mathrm{~b}$.

Development of the clingfishes Diplocrepis puniceus and

Trachelochismus pinnulatus (Pisces: Gobiesocidae). In press.

Rugh, R. 1948.

Experimental embryology. Minneapolis: Burgess Publishing

Company, $481 \mathrm{pp}$.

Runyan, S. 1961.

Early development of the clingfish, Gobiesox strumosus Cope.

Chesapeake Sci., $2(3,4)$ : 11.3-141, 33 fig., 6 tab. 
152.

Tavolga, W. N. and Rugh, R. 1947.

Development of the platyfish, platypoecilus maculatus.

Zoologica, N.Y., 32: 1-15, 25 fig.

Weisel, G. F. 1947.

Breeding behaviour and early development of the mudsucker,

a gobiid fish of California. Copeia, 2: 77-85, $10 \mathrm{fig.,} 1 \mathrm{tab}$. 
153.

Fig. 1. Forsterygion varium. No.1: I hour; 2:7-8 hours; 2a: 9-10 hours; 3 : 18 hours; $4: 42$ hours; $5: 3$ rd day; 6: early 4 th day; $7: 4$ th-5th day; $8: 7$ th day; $9: 9$ th day: 10: 11th day; 11 ; 14 th day; $12: 18$ th day. 


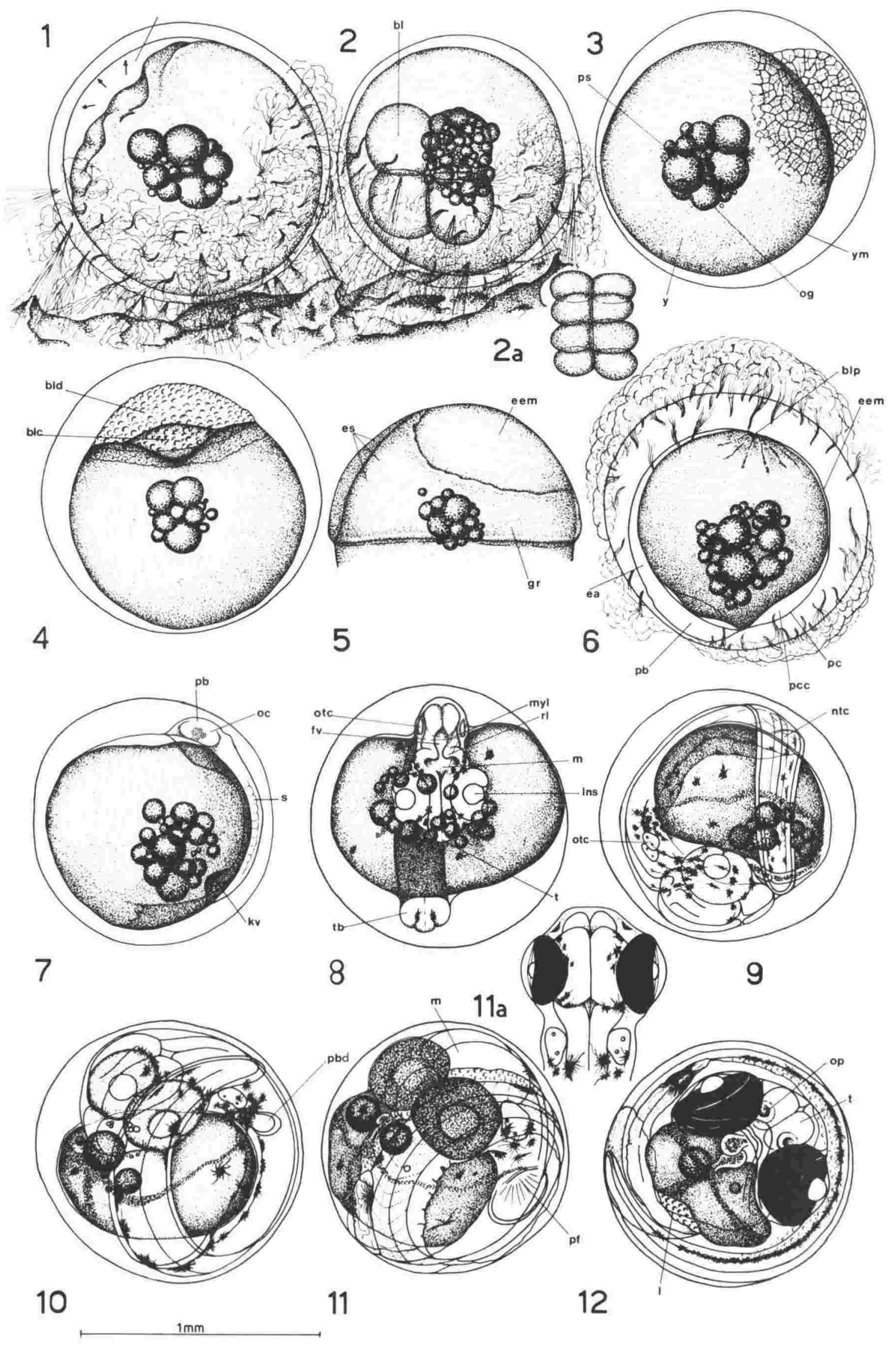


Fig.2. Forsterygion varium. No's 1 \& 2: prolarva. Abbreviations: an, anus; at, atrium; bl, blastomere; blc, blastocoel; bld, blastula-blastoderm; blp, blastopore; cf, caudal fin; cl, cleithrum; df, dorsal fin fold; ea, embryonic axis; eem, extra-embryonic ectoderm es, embryonic shield; fv, 4th ventricle; $g$, gut; gb, gallbladder; gr, germ ring; kv, kuppfers vesicle; 1, liver; $1 j$, lower jaw; lns, lens; $m$, mesencephalon; mt, metencephalon (cerebellum); myl, myelencephalon; myt, myotomes; ntc, notochord; oc, optic cup; og, oil globule; op, olfactory pit; otc, otic capsule; otl, otoliths; pb, primordial brain; pbd, pectoral bud; pc, pericardium; pcc, pericardial cavity; pf, pectoral fin; pp, peritoneal pigment; prt, protoplasm; ps, pigment sphere, rl, rhombic lip; s, somite; sc, spinal cord; $s v$, sinus venosus; $t$, telencephalon; $t b$, tail bud; $v$, yentricle; vf, ventral fin fold; vp, ventral melanophores; y, yolk; ym, yolk membrane. 

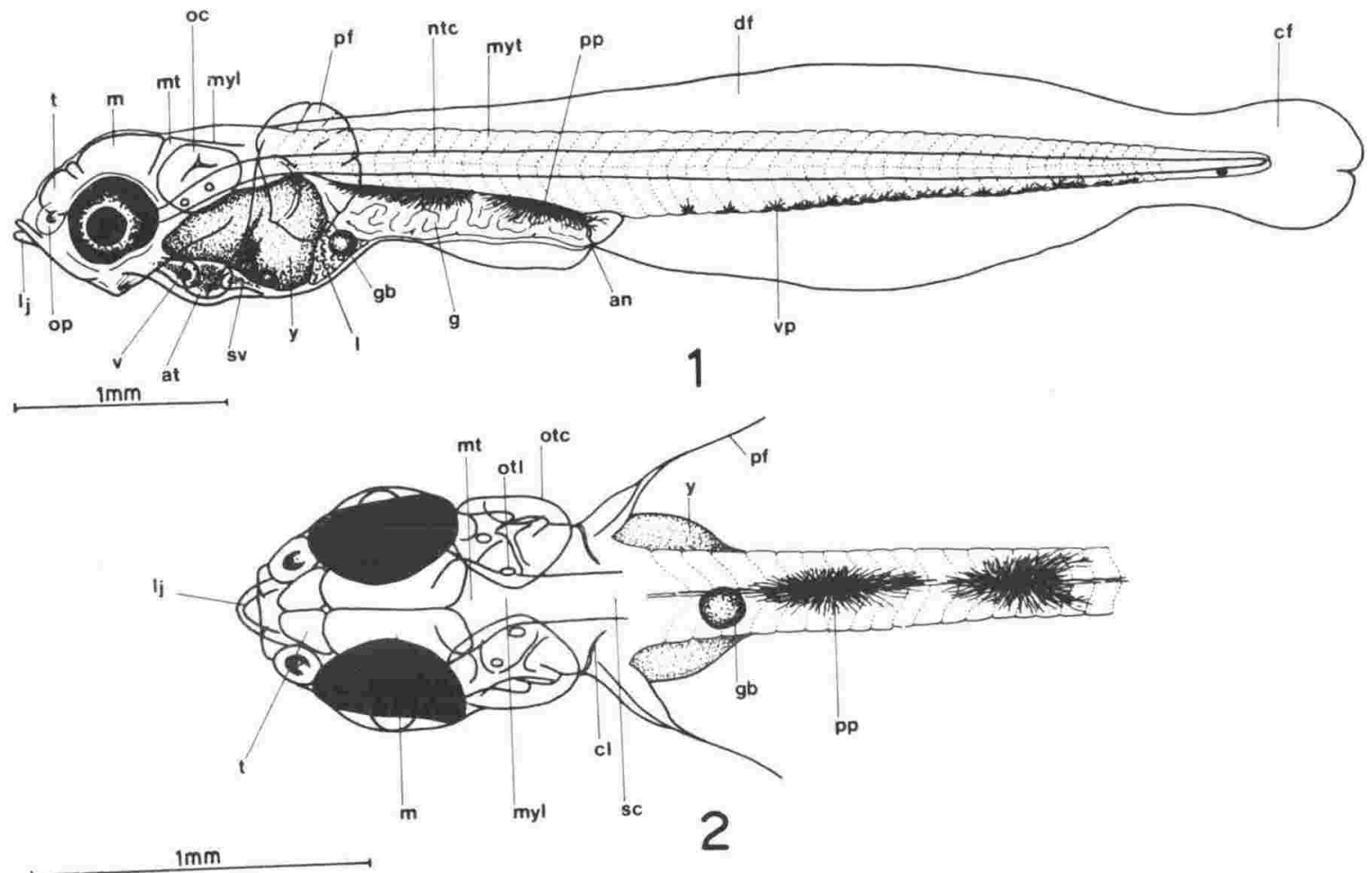
Fig.3. Gilloblennius decemdigitatus. No.1: $30-40$ hours; 2: 3 rd day; $3: 6$ th day; $4: 7$ th day; $5: 8$ th day; 6: 10th day; 7: 13th day; 8: 14th day. 

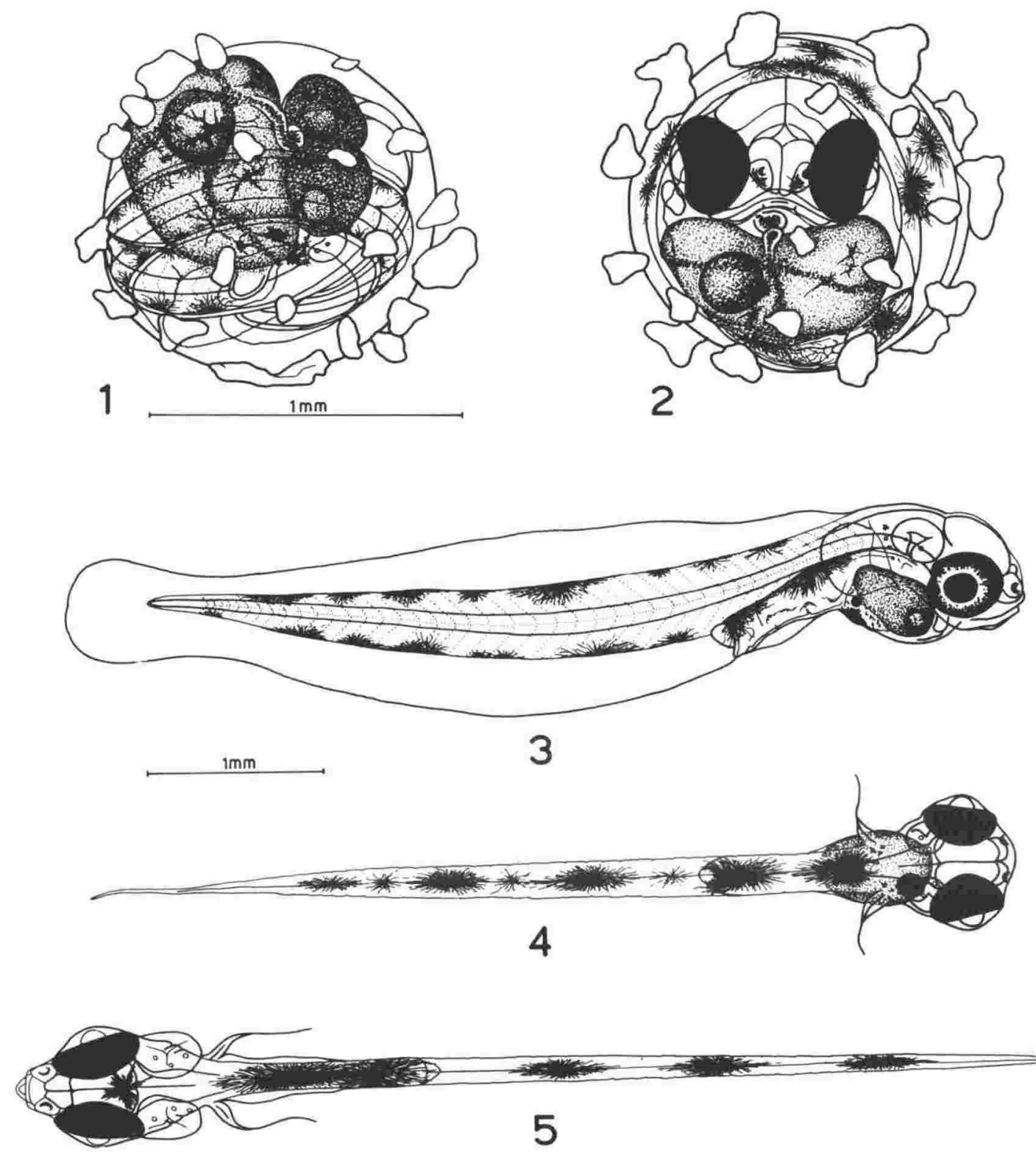

5




156.

Fig.4. Gilloblennius decemdigitatus. No.1: 15th day; 2: 20th day; 3 \& 4: prolarvae, 5 \& 6: 5 day old prolarva, $5.80 \mathrm{~mm}$ s. 1 . 

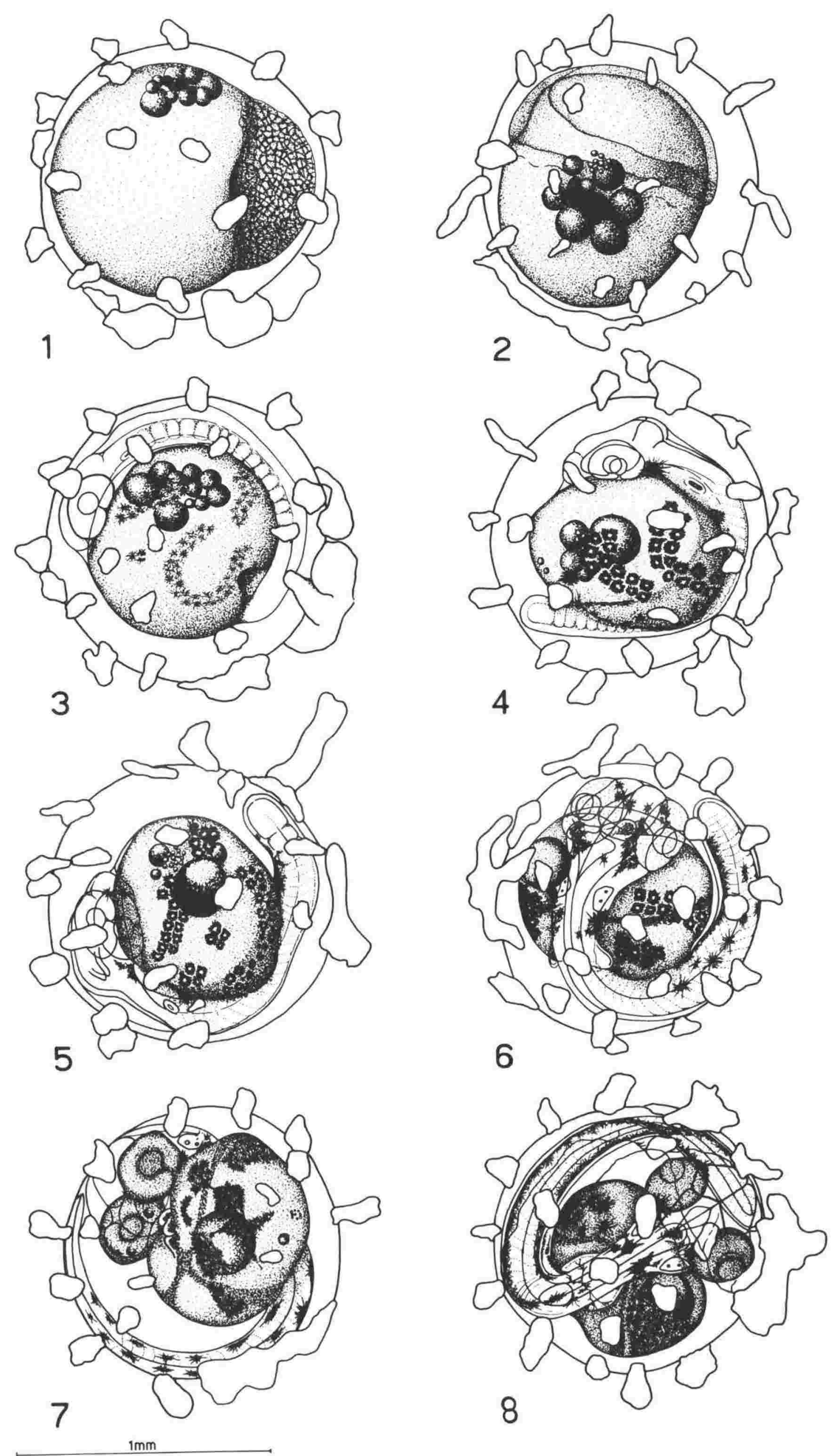
Embryological development of Gilloblennius tripennis (Forster, in Bloch and Schneider, 1801) (Pisces: Tripterygiidae)

By J. G. Ruck

Department of Zoology, Victoria University, Wellington

Abstract

In the Wellington area the eggs of $G$. tripennis are laid from July to October on the undersurface of stones and shells in shallow tidal zones. The eggs are spherical (1.32-1.40 $\mathrm{mm})$ and have large yolks (1.20-1.31 mm). Each yolk contains a large oil globule, several smaller ones and aggregations of melanic pigment. The chorion has about 12 adhesive tendrils that radiate from specific points and intertwine with adjacent eggs. Development to hatching takes about 21 days at $13-15^{\circ} \mathrm{C}$.

The prolarva is 5.40-6.00 mm standard length with a large yolk sac. There are two melanophore patches above the gut and a series of 0-9 melanophores along the ventral mid-line of the tail. One medium sized melanophore lies above the optic lobes and a further two spidery ones above the medulla.

\section{Introduction}

Gilloblennius tripennis (Forster in Bloch and Schneider, 1801) is an endemic tripterygiid which lives in areas of rock and algae along the New Zealand coast. This species is seldom found in tide pools, preferring sub-littoxal zones or large semi-enclosed areas permanently open to the sea. Specimens I have seen have always been brightly coloured, blending well with rock surfaces encrusted with sponges, anemones and seaweeds. The colour consists basically of 6 dark green H-shaped patches along the doxsal mid-line, each one fringed by a lighter green line. The area between the patches is a uniform red to mauve colour and the doxso-lateral aspect of the head is mottled in 
greens and purples. The ventro-lateral zones behind the anus consist of alternating bands of black and light green, contrasting with the whitish abdomen. The anal fin is frequently a faint orange colour. These colours apply to Wellington specimens.

The distinctive colours and overall lower fin-ray counts distinguish this species from the closely related Gilloblennius decemdigitatus (Clarke, 1879), the embryological development of which is described elsewhere (Ruck, in press).

\section{Materials and Methods}

Only three egg clusters were found, two at Lyall Bay on 1.9.71, 27.7.72 and one at Breaker Bay on 6.8.72, all in the Wellington area. Those collected from Lyall Bay were at a late stage of development and nearly all the prolarvae had hatched by the time the eggs had arrived at the Marine Laboratory. The cluster collected on 6.8 .72 had eggs at varying stages of development, some as early as the "four-cell" stage, while others were close to hatching. No male or female parent was collected with the eggs and therefore the conclusion that these eggs belong to Gilloblennius tripennis relies heavily on circumstantial evidence. The eggs from Breaker Bay were found in an Haliotis (Paua) shell that was wedged between two rocks at such an angle as to partially expose the eggs within (Fig.1, no.1). An attendant adult lay above the eggs and a tentative identification as G. tripennis was possible.

Furthermore the eggs have a characteristic pattern of pigment cells on the yolk surface (Fig.2, no.2), which I have so far found only on these eggs and those of G. decemdigitatus (Ruck, in press). Finally, the time the larval. stages appear in the plankton coincides with the months when the eggs were found. During the months of July, August and September I searched the shallow water (up to 20') around Breaker Bay, Island Bay and lyall. Bay but found no more specimens.

The eggs were kept in the laboratory in small plastic aquaria supplied with running seawater and an air supply that passed aerated water over the eggs (Ruck, in press). Eggs required for observation and illustration were removed from the shell by a blunt needle and large pipette Sketches of the embryological development were made using 
lucida, and an ocular micrometer was used for measuring eggs and larvae. Measurements of the prolarvae and the terminology of the erbryological stages are the same as those of Ruck (in press).

\section{Spawning and development}

The main spawning time, as indicated by the presence of a large number of larvae in the plankton, is during July, August and september with a gradual decline in october. Information relating to the identification of later larval stages, their relative proportions and numbers, will be included in a later publication dealing with several tripterygiid larval species. The 3 clusters of eggs were found below low tide in areas of considerable rock and algal cover. Two were confined in the inner surface of large Haliotis shells while the third occupied an indentation on the undersurface of a coralline encrusted rock. In each case the eggs formed a multi-layered pad of cells that spread outward as a single layer at the periphery. The eggs occupied the deepest, most protected part of the inner shell (Fig.1, no.2). With this arrangement of the eggs aeration (under laboratory conditions) of the innermost ones was difficult resulting in a high mortality from bacterial and fungal infection. Under more natural conditions these eggs would require a constant supply of fresh water coupled with the intimate attention of one paxent, a condition characteristic of many New Zealand tripterygiids (Ruck, in press).

\section{Developinent of the egg}

The eggs in situ are pale yellow, although some of those more advanced in development appear slightly orange. The eggs are entwined in tendrils which impart a greyish hue to the clusters. The deepest layer of eggs displays the tiny black eyes and yolks of the advanced embryos. The most recently laid eggs lie at the edges of the clusters. It is evident that the clusters composed of several thousand eggs at varying stages of development are produced by successive and different spawnings.

Eggs early in development are spherical (1.32-1.40 mm diameter) and have large yolks (1.20-1.31 mm diameter) which occupy most of the inner chorionic cavity. 
Within each yolk there is a large oil globule (0.33-0.35 mm) partially surrounded by and closely associated with 5-9 irregular masses. Each mass is composed of melanic pigment and numerous minute oil spheres. One or two other medium sized oil globules may be found in the yolk separate from the main one.

Radiating from specific points on the chorion are tracts of elastic filaments (approx. 12 per egg), which intertwine with those of adjacent eggs, forming a framework within which the eggs are suspended. Many of the eggs suspended thus make no chorionic contact with others. The clusters are satisfactorily resistant to shock and remain porous enough to allow movement of water around each egg. The tendrils of the lowermost eggs adhere to the substrate and thus effectively anchor the whole pad of eggs.

Development to hatching (Fig.l and 2, no's 3-12) took 21 days at a temperature of $13-15^{\circ} \mathrm{C}$.

First day (Fig.1, no.3). The groups of eggs from the edge of the main cluster were at various stages of early development. The earliest consisted of 4 transparent, rounded blastomeres raised prominently from one end of the yolk. Eight blastomeres are formed by the division of the 4 blastomeres at right angles to the yolk surface. The result is two rows of 4 cells lying close to the yolk surface.

Second day (Fig.1, no.4). Approximately 15 hours after the 8 blastomere stage, the early blastula is formed. This comprises a prominent cap of cells (blastoderm) that overlies a rather shallow blastocoel. Individual cells are faintly discernible and constitute the blastoderm. These cells are collectively referred to as the blastodisc.

Third day (Fig.I, no's 5a, 5b \& 5c). Early on the third day (20 hours later) the blastodisc has spread almost a third of the way around the yolk. The germ ring is preceded in its migration by a monolayer skirt of cells. The embryonic shield develops at the point of greatest involution. The pigment masses about the large oil globule have become slightly more diffuse and attenuated. Late in the third day the blastodisc covers two thirds of the yolk surface. The embryonic shield is thicker and longer encircling almost a quarter of the yolk circumference, The central portion of this shield is thicker and notches into the yolk especially at the uppermost end (no. $5 \mathrm{c}$ ), the site of the future brain. A refractive 
streak is visible running down the centre of the shield. This is the neural keel or future central nervous system.

Fourth day (Fig.1, no's $6 \mathrm{a} \& 6 \mathrm{~b}$ ). The blastodisc covers all but a small portion of the yolk (the blastopore). The embryonic axis (notochord, neural keel and somites) is very well defined and encircles almost half of the yolk. The central nervous system is well defined, especially the brain where the optic vesicles have budded from the prosencephalon (6a). Six myomere blocks lie lateral to the spinal cord. Lateral to the head of the embryo on the surface of the yolk and within the extra embryonic ectoderm are faint tiny melanophores. These are arranged in twisted patterns. The hind most portion of the embryo is only slightly raised above the contour of the yolk.

Fifth day (Fig.2, no.7). The blastopore, represented by a small indentation at the end of the tail, is closed. The embryo encircles more than half of the yolk, and is raised above the yolk contour along its entire length. The brain has expanded laterally and dorsally and shows signs of lobe formation. The ectodermal lens are quite flat and are only partially enclosed by the optic cups. Shallow depressions in the ectoderm lateral to the medulla mark the auditory placodes. Eleven pairs of myomeres are present. Within the embryo pigmentation is limited to several diffuse melanophores behind each eye and each auditory placode. The pigmentation on the yolk surface is more extensive, consisting of several groups of up to 70 melanophores. The groups lie in close pxoximity to the anterior part of the embryo, forming in some cases twisted patterns. The individual pigment cells are rather diffuse at this point. The pignent about the large oil globule continues to dissipate, at a rate proportional to the build up of pigment at the yolk surface.

The pigment cells present in embryonic and larval fishes are shown by orton (1953) to originate from the neural crest. These cells are amoeboid and migrate to various parts of the body. They may be pigmented soon after Jeaving the neural crest (clupeids, labrids, scombrids etc.) or later at their eventual destination (girellids, engrauljds, cyprinids etc.).

The gobiesocids conform with the latter, because the pigmentation of the cells occurs after migration into areas of the pexitoneurn and tail myotomes (Ruck, 1971, 1973b). In contrast the tripterygi.ids are intermediate to the two extremes. The cells begin to pigment close to or on the yolk 
surface after coming from the neural crests and before travelling to other parts of the body. The tripterygiids typically have reservoirs of melanic pigment deep within the yolk, which diminish at a rate proportional to the uptake of pigment by cells outside the yolk (Ruck, 1973a; in press). It is presumed that the yolk reservoirs are the source of pigment for the future chromatophores. The melanophores, following pigmentation, aggregate in specific parts of the embryo in a pattern typical of many tripterygiids. These areas are about the eyes (probably later incorporated into the chorioid), behind each otic capsule, halfway along the embryo, and as a series . ventral to the tail. Those behind the otic capsules, excluding the tail series, are eventually incorporated into the peritoneum dorsal and lateral to the gut. The number of melanophores involved varies in different species.

Sixth day (Fig.2, no.8). The pexicardial cavity has expanded beneath the head, and is visible from the lateral aspect as a shallow chamber. Very faint pulsation is discernible in the region of the heart. The walls of the brain are laterally folded defining the ventricles. The cerebellum forms as the rhombic lip, or fold, on the anterior edge of the fourth ventricle. The lenses are spherical and occupy the whole of the optic cups. The olfactory pits lie wedged between the eyes and the telencephalon. Further rows of faintly melanic pigment cells border the inner curvature of the eyes between the latter and the brain. The melanophores behind the eyes and otic vesicles are better defined and much darker. Individual melanophores within the groups on the yolk surface are angular and make contact with adjacent pigment cells along straight borders. No interdigitating of melanophore extensions is obvious at this stage. The superficial yolk pigment has spread away from the head further around the yolk. Several large isolated melanophores lie scattered over the yolk surface. The central oil globule is now devoid of associated pigment or oil droplets, and has decreased in size. The anterior miyomeres axe chevron shaped whilst those that extend into the tail remain block shaped. The tail bud has not lifted clear of the yolk.

Seventh-Eighth day. The heart is tubular, lying beneath the head, and beats regularly sending a weak flow of blood through the main vessels. The yolk is smaller and has a slightly more orange hue. This is partly due to the blood in the vitelline vessels. The chorioids of the eyes have begun to pigment beginning with an aggregation of tiny melanophores along 
a medium horizontal strip. This strip gradually spreads to cover the entire inner surface of the eye with faint pigment. The tail bud is clear of the yolk surface.

Ninth-Tenth day (Fig.2, no.9). The pericardial cavity has migrated forward of the head exposing the sinus venosus that receives blood from the large, well developed vitelline veins. The other heart chambers lie obscured beneath the head. The brain lobes, especially the mesencephalon (optic lobes), have expanded considerably and the chorioid roof has thickened. With this expansion the eyes have been pushed laterally, thus broadening the whole head. The auditory capsules contain two small granules. The lenses occupy only a portion of the optic cups, and the chorioid fissure is almost closed. The tail is well clear of the yolk and chevron shaped myomeres pass along the entire length. A narrow fin fold borders the tail. The melanophores behind each eye and otic capsule have increased in number, while those previously bordering the eyes have disappeared. A single melanophore is present in front of each eye, above the fourth ventricle and above the beginning of the spinal cord. The rounded pectoral buds are visible behind the exit of the ductus cuvieri (main lateral yolk vessels) from the body. Behind these buds the tubular gut emerges and continues a little way round the yolk. The hindmost portion of the gut is surrounded by 5-6 melanophores. The original twisted and well defined state of the superficial yolk melanophores has been superceded by a mass of interdigitating pigment cells lateral to the head. This reorganization begins by the expansion of individual nelanophores that bordered the formerly precise groups. Eventually all the yolk pigment cells respond in this manner. Very few melanophores lie above the pericardjal cavity or the vitelline veins. A single stellate melanophore is present on the ventral mid-line of the tail just past the anus.

The embryo moves frequently.

Fourteenth-Fifteenth day (Fig.2, no's $10 \& 10 \mathrm{a}$ ). The head has lifted slightly and exposes all the chambers of the heart. The chorioid is well pigmented but the lenses are still visible with transmitted light. The chorioid fissure is present as a faint white line ventral to the eyes. The broadening of the head has brought the otic capsules closer to the eyes. The overall pigmentation has altered little, except that the yolk melanophores have concentrated close to the eyes leaving much of the ventral yolk surface fxee of pigment cells. The liver is visible on the left side flattened 
against the yolk (no. 10a). The yolk melanophores scattered behind the ductus cuvieri taper to a point behind the liver and appear to pass up onto the hindgut. These possibly contribute to the melanophore patch that is present above the gut in latex larval stages.

Twentieth day (Fig.2, no.11). The embryo is ready to hatch and therefore possesses general characteristics identical to the prolarva. The embryo is crammed tightly into the chorionic cavity, giving very little room for movement. Activity is restricted to muscular flexing of the tail with the occasional gyration of the whole embryo. Movement increases prior to hatching which results in the rupture of the chorion and the subsequent release of the prolarva.

Prolarva (Fig.2, no.12). Prolarval length on hatching ranges from 5.40-6.00 mm standard length. The prolarvae are positively phototropic swimming to the most illuminated portion of the aquarium. The anterior region is bulky due to the residual yolk and blunt snout. The oil globule remains in the yolk but is very much reduced in size. The gut is elongate and convoluted. Pigmentation on the yolk consists of numerous scattered melanophores concentrated on the antero-lateral aspect, but spreading back toward the liver. The yolk is bordered anteriorly by the heart and posteriorly by the liver. The blood flow is rapid and visible in all the main vessels.

The arrangement of the majox vessels is essentially the same as for Forsterygion varium (Ruck, in press). The upper parietal peritoneum of the gut is covered anteriorly by a large melanophore patch that sends antero-lateral projections about the gut. A second smaller patch lies above the anus. A small group of stellate melanophores lies behind the eyes and each otic capsule. There is one medium sized melanophore directly above the optic lobes and a further two spidery ones (not always present) somewhat haphazardly placed above the medulla. The eyes appear fully pigmented. Along the dorsal mid-line well down toward the caudal lobe are usually two elongate melanophores. On the ventral mid-line of the tail are 0-9 smallex melanophores. The pectoral fin is merely a semi-circular flap that arises directly behind the otic capsule high up on the body.

Prolarva seventh day. A length of $6.35-6.80 \mathrm{~mm}$ s.1. is reached, with virtually all the yolk absorbed. The prolarva is more attentuated, 
has a longer snout, and is less pigmented overall. The melanophore pattern about the liver is somewhat variable, representing that remaining from around the yolk. In some cases the liver is surrounded by a single yentral pigment cell and two elongate lateral ones (one each side only). The lateral melanophores may extend upwards and backwards to partially link with the pigment above the gut. In addition to these a medium ventral row of 1-3 melanophores extend back onto the gut. Nearly any combination of these is possible: some with only one single large melanophore beneath the anterior corner of the liver and perhaps a row along the mid-line; others with only one lateral melanophore present and a complete absence of the rest. The pigment in the peritoneum above the gut has blended to form a continuous single patch.

The melanophores above the central nervous system have aligned themselves, leaving one large one above the optic lobes, one medium size above the medulla and a much smaller one above the start of the spinal cord.

The melanophores behind the eyes and otic capsule have disappeared. From 2-6 melanophores are present along the dorsal mid-line compared with 6-11 along the ventral mid-line of the tail. The caudal ray anlage is visible at the end of the tail and extends into the ventral part of the caudal fold. A small melanophore is usually present within the fin fold anterior to the caudal anlage. The gut shows minor signs of coiling.

The measurements of 25 prolarvae are as follows:

$\begin{array}{lcc} & \text { Range } & \text { Average } \\ \text { Standard length (mm) } & 5.40-6.00 & 5.72 \\ \text { Total length (mm) } & 5.50-6.20 & 5.93 \\ \text { Snout-vent (rm) } & 2.00-2.40 & 2.20 \\ \text { Head length (mm) } & 0.95-1.15 & 1.05 \\ \text { Eye length (mm) } & 0.45-0.55 & 0.50 \\ \text { Greatest depth (mm) } & 0.95-1.30 & 1.1 .0 \\ \text { Dórsal mid-line pigment } & 2 & 2 \text { most common } \\ \text { Ventral mid-line pigment } & 0-9 & 0 \text { most common } \\ \text { Myotomes } & 38-40 & 40 \text { most common }\end{array}$


166.

The measurements of 4 seven day old larvae are as follows:

$\begin{array}{lllll}\text { Standard length (mm) } & 6.80 & 6.40 & 6.35 & 6.35 \\ \text { Total length (mm) } & 7.10 & 6.60 & 6.60 & 6.55 \\ \text { Snout-vent (mm) } & 2.60 & 2.50 & 2.35 & 2.25 \\ \text { Head length (mm) } & 1.30 & 1.2 & 1.2 & 1.2 \\ \text { Eye length (mm) } & 0.55 & 0.55 & 0.55 & 0.55 \\ \text { Greatest depth (mm) } & 0.95 & 0.90 & 0.90 & 0.85 \\ \text { Dorsal mid-line pigment } & 6 & 3 & 0 & 3 \\ \text { Ventral mid-line pigment } & 11 & 6 & 9 & 7\end{array}$

\section{Literature cited}

Orton, G. L. 1953.

Development and migration of pignent cells in some teleost fishes. J. Morph., 93: 69-99, 25 fig., 5 pl.

Ruck, J. G. 1971.

Development of the lumpfish Trachelochismus melobesia (Pisces:

Gobiesocidae), Zool. Publs Vict. Univ. Wellington, 57: 1-9, 4 fj.g.

Ruck, J. G. 1973a.

Development of Tripterygion capito and Tripterygion robustur

(Pisces: Tripterygiidae). Zool. Publs. Vict. Univ. Wellington,

63: 11 pp., 3 fig.

Ruck, J. G. $1973 \mathrm{~b}$.

Development of the clingfishes Diplocrepis puniceus and

Trachelochismus pinnulatus (Pisces: Gobiesocidae). Zool. Publs

Vict. Univ. Wellington, 64 : $12 \mathrm{pp} ., 4 \mathrm{fig}$.

Ruck, J. G. (in press). The development of Forsterygion varium and Gilloblennius decemdigitatus (Pisces: Gobiesocidae). J. mar. Freshwat. Res. 
167.

Fig.1. Gilloblennius tripennis. No.1: Haliotis shell with eggs, wedged between rocks; 2: Pad of eggs inside Haliotis shell, 3: 4-cell, 8-cell and gastrulation stages; 4 ; 2 nd day; $5 a, 5 b$ \& $5 c$ : $3 r d$ day; $6 a \& 6 b: 4$ th day. 


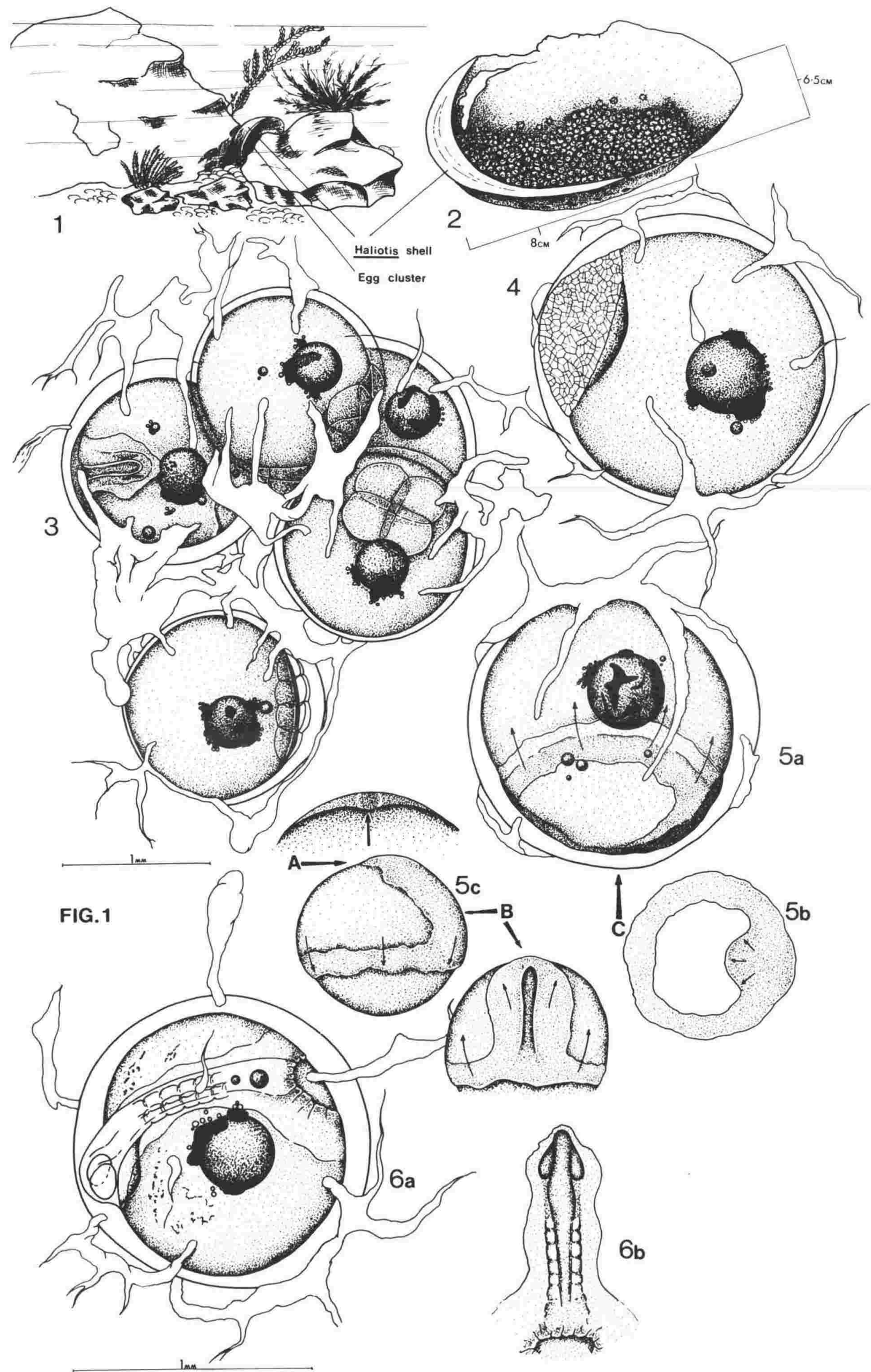


168.

Fig.2. Gilloblennius tripennis. No.7; 5th day; $8: 6$ th day; 9: 9th-10th day; 10 \& 10a: 14 th-15th day; 11 : 20th day; 12: Prolarva. 


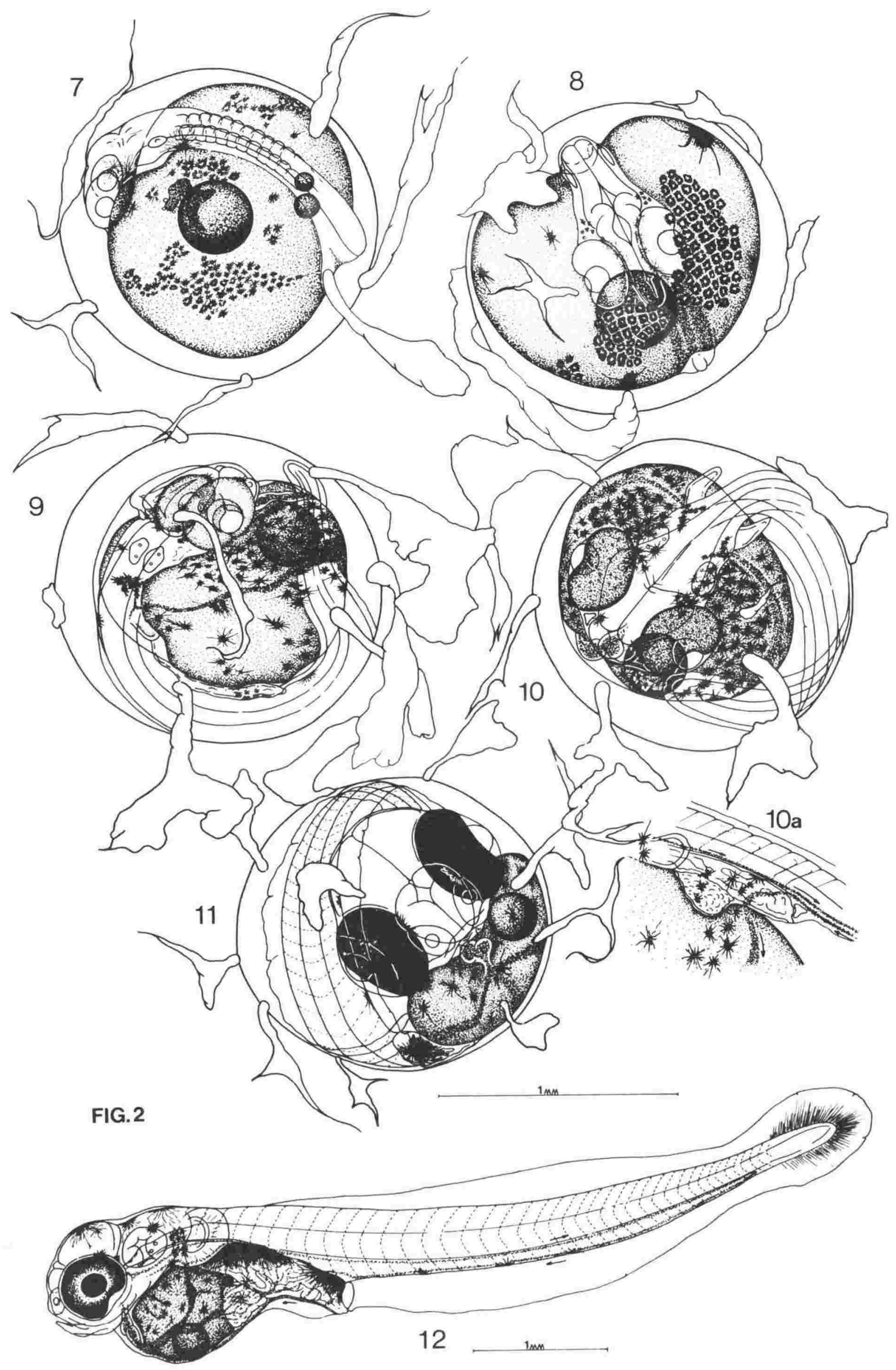




\section{Discussion - embryological development}

The eggs of nine species of New Zealand fishes common to inshore areas are described and figured for the first time. The method of presentation is one that is commonly used (Dotu and Mito, 1955; Mito, 1954, 1963; Cassie, 1956, 1963; Swarup, 1958; Runyan, 1961; Deuel et al.. 1966; Benzie, 1968c, 1968d; Ritchie, 1969; Baker, 1971, 1972; Robertson and Raj, 1971; Llewellyn, 1973, 1974; Miller and Sumida, 1974; Berrien, 1975), where series of diagrams or photographs depicting stages in the embryological development are presented along with detailed descriptions of each stage.

This embryological work extended over several years, and when the study of each egg series was completed it was published. The order in which the species were dealt with depended principally on the availability of material rather than choice. Some of the publications pre-date work done on the taxonomic review of the New zealand tripterygiids and clinids. In combination these facts explain two anomalies in the embryological section, these being firstly the separation of the accounts of congeneric species (the early stages of Trachelochismus melobesia and I. pinnulatus appear in separate papers), and secondly the inconsistency in the nomenclature of two tripterygiid species (Tripterygion capito in the embryological section is subsequently referred to Forsterygion and Tripterygion robustur in the embryological section is later synonymized with Forsterygion nigripenne).

The species in the order in which they have been studied are, Trachelochismus melobesia Phillipps, 1927; Forsterygion capito (Tripterygion capito) (Jenyns; 1842); Forsterygion nigripenne (Tripterygion robustum) (Valenciennes in Cuvier and Valenciennes, 1836); Diplocrepis puniceus (Richardson, 1846); Trachelochismus pinnulatus (Forster in Bloch and Schneider, 1801); Forsterygion varium (Forster in Bloch and Schneider, 1801); Gilloblennius decemdigitatus (Clarke, 1879); G. tripennis (Forster in Bloch and Schneider, 1801) and Gastroscyphus hectoris (Gunther, 1876). The last mentioned species is illustrated in the larval and not the embryological section of this thesis.

There has been relatively little work published on the spawning and egg development of the New Zealand tripterygiids, clinids and gobiesocids. Graham (1939, 1953) describes the spawning behaviour and figures the embryological and prolarval stages of a species he named as Tripterygion 
varium. However, the present confusion in tripterygiid nomenclature does not allow me to determine which species Graham (1939, 1953) was describing. The diameters of the eggs he describes do not match those of Forsterygion varium or $\mathrm{F}$. nigripenne but although somewhat low they do approach those of F. capito. The prolarval descriptions are also too general to allow precise identification. Darby (1966) describes the spawing behaviour and prolarval stage of Helcogramma medium (Gunther, 1861). The taxonomic status of H. medium is confused (see review), and there is some evidence that Darby (1966) may have dealt with several species rather than one. The larval work on tripterygiids has revealed at least two and possibly three new Helcogramma species which are meristically similax and likely to be common throughout the tidal. zone (prejuveniles abundant in the surge channels). Furthermore Gunther (1861) in describing H. medium uses specimens with 20-21 anal rays and others with 23. Evidence from the meristics of the prejuvenile Helcogramma species indicates that Gunther, by using specimens with different anal ray counts, has described at least two species for $\mathrm{H}$. medium.

The only account of New Zealand clinids is that by Moreland and Dell (1950) where they describe developing eggs in the abdomen of Ericentrus rubrus (Hutton, 1872). Ericentrus rubrus is ovoviviparous and gives bixth to small larvae that have developed well past the yolk sac stage. The eggs are oval, without adhesive tendrils, and superficially resemble those of the gobiesocid $\underline{T}$. pinnulatus. The embryological development is typical of that found in oviporous tripterygiids e.g. F. varium.

The gobiesocids have received more attention, with the eggs and larvae of $\mathrm{D}$. puniceus described by Graham (1939, 1953) and the spawning, egg masses and prolarvae of T. pinnulatus described by Coakley (1964). There is no doubt that the eggs described by Grahan (1939, 1953) belong to D. puniceus, but the poor quality of the illustrations and descriptions make them of limited value for embryological comparison. Information on the development of other New Zealand marine and freshwater species is more extensive although somewhat scattered, as shown in the annotated bibliography in the appendix. Some of the more informative accounts are those of Anderton (1906); Thomson and Anderton (1921); Graham (1939, 1953); Cassie (1956, 1963); McKenzie (1961); McDowall (1965); Benzie (1968c, 1968d); Ritchie (1969); Baker (1.971, 1972, 1973); Robextson and Raj (1971); Robertson. (1973, 1975). 
Several New Zealand tripterygiids and gobiesocids e.g. F. varium and $T$. pinnulatus, have large eggs which are readily available from the shore and easily maintained in laboratory aquaria (Ruck, in press a). The large size and clear chorion of gobiesocid eggs make them particularly suitable for in vivo study of developing embryos. All the species observed in the present study displayed parental care of the egg stages, by fanning the clusters with their fins and by aggressively opposing intruders. The prolarvae emerge with reduced yolk sacs and functional jaws so that feeding begins soon after hatching. The fecundity is low (20-3000 eggs) compared with that of pelagic fishes which may produce scores of thousands of eggs (Breder, 1962; Ryland, 1966; Smith,1972; May, 1975). With parental care in the tripterygiids and gobiesocids, a significant proportion of the early development takes place in relative security, thus offsetting the low fecundity. Many other families of reef fishes show comparable parental care of demersal eggs e.g. Blenniidae, Gobiidae, Cottidae, Pholidae, stichaeidae and some Clinidae etc., (Gibson, 1969). The tendency for inshore fishes to lay fewer eggs (Gibson, 1969) but to adequately protect them during the vulnerable stages of embryological development is an advantage in the relatively severe physical conditions of coastal reef zones.

The male of the species generally "guard" the egg clusters and each must attract a series of females to his "nest" for spawning (Gibson, 1969). Associated with this are elaborate behavioural displays which are exhibited by both males and females not only for courtship but also for protection of territory i.e. "nests" and egg clusters (Breder, 1941; Weisel, 1947; Mito, 1954; Tavolga, 1956; Gibson, 1969). Elaboxate spawning behaviour of this nature was observed for $F$. varium, and was identical to that recorded for the same species by Doak (1972). However, little attempt was made to document the behaviour of the species studied because it was considered beyond the scope and aims of this thesis.

In the gobiesocids and tripterygiids egg clusters are laid on or beneath submerged objects with each egg attached to the substrate or to another by an adhesive structure. Typically the yolks of the eggs are pigmented, by substances dispersed throughout the yolk, and also within pigment cells (tripterygiids only) on the yolk surface. The colour of the yolks and the arrangement of pigmentation is diagnostic of the species. The value of brightly coloured eggs is unclear, although Breder (1962) suggests that opacity may protect labile structures or systems from 
harmful radiations by filtering or direct blocking. Breder (1.962) believes that further protection from radiation may be afforded by laying eggs in shaded areas, although protection from predation is likely to be a stronger motive. Also the habit of laying eggs in crevices and under stones undoubtedly protects eggs from direct wave action and may also improve ventilation by allowing one-way passage of water across the eggs. Because visual stimuli play an important role in courtship behaviour of fishes (Tavolga, 1956), it is possible that the coloured eggs reinforce the territorality response in the male parent attending the eggs, and may also serve as a warning to intruding fishes. In the gobiesocid D. puniceus the males were seen to assume the purple hue of the eggs being tended.

The tripterygiids and gobiesocids are typical teleosts with respect to their embryological development (Battle, 1944; Tavolga and Rugh, 1947; Rugh, 1948; Balinsky, 1965; New, 1966; Billet and Wild, 1975). The eggs follow the usual demersal pattern, with small subdermal spaces, relatively small fins on the developing embryos, prominent vitelline circulation, pigmented yolks and the hatching of well developed prolarvae (Shelbourne, 1956; Breder, 1969). Despite the above there are differences in the basic structure of tripterygiid and gobiesocid eggs. Tripterygiid eggs are typically spherical with the exception of those of F. capito which are slightly depressed. They have well developed adhesive tendrils, reservoirs of melanin within the yolks, and embryos with considerable somatic and yolk pigment cells. The chorion is often obscured by detritus attached to the tendrils. In contrast gobiesocid eggs are typically depressed, have flat adhesive bases without tendrils, and have brightly coloured yolks. The embryos and yolks, compared with the tripterygiids, are markedly devoid of pigment cells (including pigment reservoirs). The pigment cells present in embryonic and larval fishes are shown by Borcea (in Orton, 1953a) (as early as 1909) to originate from the neural crest. These cells are amoeboid and migrate to various parts of the body. The cells may be pigmented soon after leaving the neural crest (clupeids, labrids, scombrids etc.) or later at their eventual destination (girellids, engraulids, cyprinids etc.) (Orton, 1953a). The gobiesocids conform with the latter, because the pigmentation of the cells occurs after migration into areas of the peritoneum and tail myotomes. In contrast, the tripterygiids are intermediate to the two extremes. The cells begin to pigment close to or on the yolk surface presumably soon after migrating from the neural crest and before travelling to other parts of the body. The reservoixs of pigment deep within the yolk diminish at a rate proportional to the uptake of pigment by cells outside 
the yolk. It is presumed that the yolk pigment reservoirs are the source of pigment for the future chromatophores. The melanophores, following pigmentation, aggregate in specific parts of the embryo in a pattern typical of the tripterygiids. These areas are about the eyes (probably later incorporated into the chorioid), behind each otic capsule, halfway along the embryo, and as a series ventral to the tail. Such an arrangement differs from that in the gobiesocid embryos where the small number of pigment cells consist mainly of a uniform array in the dorsal peritoneum, a short series ventral to the tail, and in some species several scattered in the lateral integument above the anus.

Tripterygiid eggs are similar to those of the blenniids (Breder, 1941; Thomson and Bennett, 1953; Mito, 1954; Munro, 1955; Dutt and Rao, 1960). In the blenniids as in the triptexygiids the eggs are laid in layers beneath objects or within shells in shallow coastal waters. In both families the eggs may be spherical, subspherical or markedly depressed, and the yolks are typically pale yellow or creamy in colour. The bright orange eggs of the blenniid Omobranchus anolius (Cuvier and Valenciennes, 1836) are an exception (Thomson and Bennett, 1953). In the tripterygiids and the blennitids the yolks contain a large oil globule and several smaller ones which diminish in size as development continues, and also opaque granular material [in Dasson steadi (Whitley) (Munro, 1955)] which is apparently the source of melanin for future melanophores. In the blenniid Paraclinus marmoratus (Steindachner, 1866) (Breder, 1941) and the

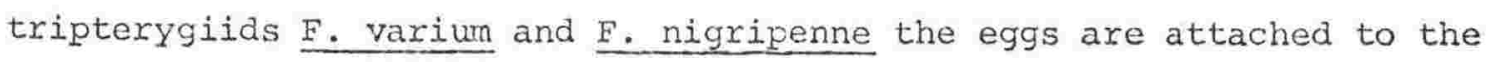
substrate by well developed adhesive tendrils. In contrast the eggs of the blenniid Omobranchus anolius (Thomson and Bennett, 1953) and those of the tripterygiid $\underline{E}$. capito are attached to the substrate by a gelatinous glue, on the flattened base of the egg, with no well defined adhesive tendrils. The overall shape of the blenniid prolarva (see above references) with a bulbous head, persistent yolk sac, pigmented parietal peritoneurn and with a mid-ventral series of melanophores along the tail is also similax to that of the tripterygiids in this study.

In contrast, however, blenniid eggs are smaller than those of the tripterygiids (0.79-0.90 mm c.f. 1.00-1.40 mm), the clusters are smaller and the period of embryonic development is shorter especially for warm water species such as Salarias enosjmae Jordon and Snyder, 1802. In the latter species embryological development takes a week at a 
temperature of $24.8-31.2^{\circ} \mathrm{C}$ (Mito, 1954) compared with 18 days for F. varium at a water temperature of about $11.5-13^{\circ} \mathrm{C}$. The New Zealand tripterygiids do not have bright red or purple pigmented bodies in the yolk as is typical of the blenniids (Munro, 1955). The prolarvae of both families vary considexably in size with those of the Blennidae being much smaller than those of the Tripterygiidae (2.85-3.70 mm c.f. $4.50-5.85 \mathrm{~mm})$. This is no doubt a reflection of relative egg sizes and relative sizes of the adults for example the maximum adult standard length of the blenniid Petroscirtes bipunctatus Day, 1875 is $55 \mathrm{~mm}$ compared with $100 \mathrm{~mm}$ for the tripterygiid $\underline{F}$. varium.

The adult blennid 0 . anolias (Thomson and Bennett, 1953) exhibits a peculiar behaviour of taking newly hatched prolarvae into their mouths and "spitting" them violently from the "nest", a behaviour not seen in the New zealand tripterygiids. This strange behaviour apparently removes a gelatinous material from axound the prolarvae, which if not removed results in the death of the prolarvae. No prolarvae of New Zealand tripterygiids are covered in this material and hence the prolarvae hatch without help from parents. In blenniids and tripterygiids the spherical eggs require the embryos to coil strongly during late development. In the Gobiids, however, the chorion is elongate and the embryos may remain uncoiled throughout development (Weisel, 1947; Dotu and Mito, 1955). According to Orton (1953b) "... a study of the larval stages of fishes and amphibians reveals much important data on their systematics and evolution." The larval form may highlight the phylogenetic relationships of a species by providing a whole new range of characteristics that can be compared (Orton, 1953b; Moser and Ahlstrom, 1970, 1972, 1974). In general terms, the more closely related are the species, the more characteristics their larval stages have in common. It is logical to assume a similar relationship for eggs and embryological stages, though it would be unwise to conclude too much about the phylogeny of the species observed in the present study because the number of species sampled is low. Nevertheless it is possible to reinforce present views on the relationship (reflected in the nomenclature) by highlighting those developmental similarities and disparities which must to some extent be a product of phyletic descent and divergence.

The tripterygiid eggs described can be separated into two main groups, equating with those of Forsterygion on the one hand and Gilloblennius on the other. Within the former group the eggs of $\mathrm{F}$. varium and $\mathrm{F}$. nigripenne 
(Tripterygion robustum) are identical in size (1.12-1.23 mm) and in yolk colour (pale yellow). The tendrils are similar and melanophores arise in the same well defined regions of the embryonic body during development. The newly hatched prolarvae are also very similar differing only in myomere numbers and the number of melanophores along the ventral midine.

The eggs of F. capito (Tripterygion capito) are also similar but differ from those of $\mathrm{F}$. varium and $\mathrm{E}$. nigripenne in theix smaller chorion diameter (1.10 mm c.f. $1.20 \mathrm{~mm}$ ), in being dorsoventrally depressed, and in having fewer adhesive tendrils on the ventral surface. The similarities of the eggs of the three species support the earlier inclusion of capito and nigripenne within Forsterygion along with varium. In the Gilloblennius group the eggs of $\mathrm{G}$. decendigitatus and $\mathrm{G}$. tripennis are spherical, the chorion is evenly covered in adhesive tendrils and the yolks have sinusoidal patterns of melanophores on their surfaces. The two species differ in the way the eggs are laid and in the pigmentation of the prolarvae. Gilloblennius decemdigitatus lays its eggs as flat, single layered sheets and each tendril on the chorion (except those close to the substrate) is a single contracted mass. In contrast the eggs of $\mathrm{G}$. tripennis are laid as multi-layered pads, with adjacent eggs intercomnected by elongate many-armed tendrils. The tendrils of $\mathrm{G}$. decemdigitatus may be contracted because the eggs are laid in sheets and do not require the extended tendrils to enmesh and keep apart neighbouring eggs, as is necessary in the multi-layered clusters. The multilayered masses are satisfactorily resistant to shock and remain porous enough to allow movement of water around each egg. The tendrils of the lowermost eggs adhere to the substrate in the same manner as for G. decemdigitatus and thus effectively anchor the whole pad of eggs.

It is possible that $\mathrm{G}$. decemdigitatus evolved from a form which laid its eggs in the same manner as does G. tripennis. If so the change from a multi-layered condition to that of a single layer would mean that the tendrils on the side of the chorion away from the substrate would no longer be necessary for anchoring eggs. In the blenniids, which are considered more specialized than the tripterygiids (Hubbs, 1952), the tendrils are restricted to the lower surface of the eggs which are laid in single layered sheets (Thomson and Bennett, 1953). This suggests that the single layered, as compared with the multilayered egg cluster, is the more primitive condition.

Forsterygion species, with the exception of $\mathrm{F}$. varium, have eggs with tendrils restricted to the ventral and ventrolateral surfaces. Here the tendrils attach directly to the substrate and rarely to adjacent eggs. 
This condition may have evolved from that found in Gilloblennius whose adults are considered slightly more primitive than Forsterygion species because of their elongate undivided lateral lines (Whitley and Phillipps, 1940). During this evolution the tendrils which were no longer used would gradually be lost. Forsterygion varium has retained the "primitive" uniform covering of tendrils, possibly because its atypical habit of laying eggs exposed to wave action requires all the tendrils for anchoring them.

The prolarvae of Gilloblennius are quite different to those of Forsterygion. Those of G. decemdigitatus are elongate and have large melanophores along the dorsal and ventral mid-lines, contrasting with the more robust and poorly pigmented prolarvae of G. tripennis. These basic differences and others (see later larval section) between these Gilloblennius species are expressed throughout the larval development indicating that not only is Gilloblennius distinct from Forsterygion but the two Gilloblennius species are, themselves quite divergent.

The egg series of 3 gobiesocid species are described in detail and have characters which, in general, support the systematic relationships established elsewhere for this group (Briggs, 1955). Briggs describes eight adult New Zealand gobiesocids. He considers that all of these except Trachelochismus pinnulatus and $\mathrm{T}$. melobesia are relicts or unrelated to any other clingfishes. He maintains that the New Zealand clingfishes were separated from the original Western Pacific stock and have been maintained by isolation. Trachelochismus pinnulatus and T. melobesia are closely related, although distinct from other New Zealand genera and species. The eggs of these two species are nearly identical in size, shape and mode of development. They differ in yolk colour (variable in

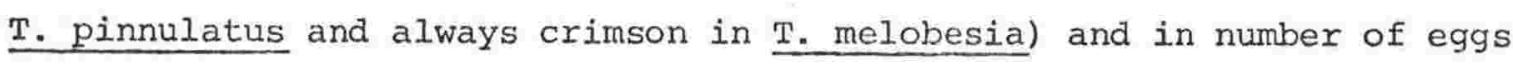
per clustex (much higher in T. pinnulatus). This difference in size of cluster relates indirectly to the size of the adults which in turn affects their fecundity. Adult T. pinnulatus (max. $71 \mathrm{~mm} \mathrm{s.1.)}$ are larger than adult T. melobesia ( $\max .30 \mathrm{~mm}$ s.1.).

The prolarvae of these two species are also very similar, differing only in size and the number of post-anal melanophores.

Diplocrepis puniceus is a relict species, whose eggs are very different from those of the Trachelochismus species. The eggs are round (c.f. oval in Trachelochismus) and depressed. The yolk is a deep purple, and there are depressions in the lateral chorion produced by contact with 
adjacent eggs. The prolarvae and late embryos are characterized by a broad series of melanophores in the integument lateral to the trunk and tail (not present in equivalent stages of Trachelochismus). Unfertilized but water hardened eggs of Gastroscyphus hectoris axe sub-triangular in shape, smaller than those of T. pinnulatus, T. melobesia and D. puniceus, and have small spherical yolks. The marked differences in eggs of Trachelochismus, Diplocrepis and Gastroscyphus support the conclusion by Briggs (1955) that these clingfish are unrelated. At the other end of the scale close relationships between adults (e.g. T. melobesia and T. pinnulatus) are strongly reflected by the similarities in the early stages.

Artificial key to the tripterygiid and gobiesocia eggs described in this study

1(10) eggs spherical or dorsoventrally flattened; yolk pale and containing pigment reservoirs; well developed tendrils usually present:

\section{TRIPTERYGIIDAE}

2(5) eggs spherical; a sinusoidal pattern of melanophores on yolk surface; chorion covered in globular or branched tendrils:

GILLOBLENNIUS Whitley and Phillipps, 1940

3(4) eggs laid as a single sheet; average chorion diameter $1.1 .7 \mathrm{~mm}$; tendrils globular:

\section{Gilloblennius decemdigitatus (Clarke, 1879)}

4(3) eggs laid as multilayered pads; average chorion diameter $1.36 \mathrm{~mm}$; tendrils elongate and branched:

\section{Gilloblennius tripennis (Forster,}

in Bloch and Schneider, 1801)

5(2) eggs usually spherical but may be dorsoventrally flattened; no sinusoidal patterns of melanophores on yolk surface; tendrils diffuse and usually restricted to the lower portion of the chorion: 
$6(9)$ eggs spherical; chorion diameter $1.12-1.23 \mathrm{~mm}$; point of attachment of tendrils to the chorion clearly defined;

7 (8) tendrils arise from all of surface of chorion; eggs laid on exposed rock surfaces under water:

Forsterygion varium (Forster. in Bloch and Schneider, 1801)

8(7) tendrils only on lower surface of the chorion; eggs laid beneath submerged objects :

Forsterygion nigripenne (Tripterygion robustum)

(Valenciennes in Cuvier and Valenciennes, 1836)

9(6) eggs dorsoventrally flattened; chorion diameter 1.00-1.10 mm; point of attachment of tendrils to the chorion poorly defined:

Forsterygion capito (Tripterygion capito) (Jenyns, 1842)

10 (1) eggs subspherical, oval or triangular, dorsoventrally flattened; yolk brightly coloured and lacking pigment reservoixs; no clearly defined tendrils:

\section{GOBIESOCIDAE}

Il(14) eggs oval; yolk white, orange, pale pink, crimson-red or pale yellow:

TRACHELOCHISMUS Brisout de Barneville, 1846

12 (13) yolk typically crimson-red or pale pink; avexage chorion dimensions $1.65 \times 1.35 \mathrm{~mm}$; egg masses raxely exceed 500 eggs:

Trachelochismus melobesia Phillipps, 1927

13(12) yolk white, orange, pale pink or pale yellow, seldom crimson-red; average chorion dimensions $1.81 \times 1.48 \mathrm{~mm}$; egg masses typically exceed 500 eggs:

Trachelochismus pinnulatus (Forster, in Bloch and Schneider, 1801) 
14 (11) eggs subspherical or triangular; yolk vivid to pale purple or pale yellow:

DIPLOCREPIS Günther, 1861 and GASTROSCYPHUS Briggs, 1955

15(16) eggs subspherical with chorionic depressions (pressure from adjacent eggs); yolk vivid to pale purple:

Diplocrepis puniceus (Richardson, 1846)

16(15) eggs triangular; yolk pale yellow:

Gastroscyphus hectoris (Günther, 1876) 


\section{Prolarvae, larvae and prejuveniles \\ Introduction}

The following section deals with the prolarval, larval and prejuvenile stages of tripterygiid, clinid and gobiesocid fish.

It is often difficult to identify fish larvae. This is due, mainly, to the marked metamorphosis which occurs between the prolarval and adult stages. An accompanying feature is that metamorphosed individuals frequently have distinctive characteristics which are absent in the larvae. The problem is accentuated where several closely related and often morphologically similar species are being compared.

The identification of larvae has, in practice, been approached in two main ways (Ahlstrom, 1975). The most difficult of the two, yet most positive, is that of laboratory rearing. The eggs from the plankton or from known parents (artificial spawning) are raised in the laboratory through metamorphosis. Characteristics of the eggs and larvae at various stages are then recorded. Marine teleosts are not easily reared but there has been success with some species, as listed by Atz (1964) and May (1970). The advantage of this technique is obvious, as definite identification of the larval stages can be made. However, it is time consuming and exacting work, often requiring elaborate equipment (Blaxter, 1962, 1968a; Fahy, 1964; Shelbourne, 1963, 1964; Shelbourne et al., 1963; Riley and Thacker, 1963; Ryland, 1966, Delmonte, 1968; Adron et al., 1974; Miller and Sumida, 1974).

The alternative and perhaps most widely used method is to build up larval series with specimens collected from the plankton. The various stages are linked by one or several relatively stable characteristics or by a distinctive trait whose gradual change may be traced without difficulty. The accuracy of this method depends on the distinctiveness of the species as well as the stability of the diagnostic features. For example characteristic fish like the sea horses (Young, 1927), trigger fish (leatherjackets) and porcupine fish (Elder, 1966) are easily recognized as they quickly acquire adult features.

To a lesser degree accuracy of larval identification may depend on geographical distribution. It is easier to determine the larval series of species restricted to lakes, estuaries or harbours where the total number of species is limited, than to establish one that is widespread and mixed with populations of many other closely related forms e.g. in the open sea. 
For the most part larval stages of teleosts are similar, and diagnostic characters are generally subtle. Characters used by researchers have included branchiostegal counts (Richards and McBean, 1966), pyloric caeca counts (Applegate, 1966), gillrakers and teeth (Ahlstrom and Counts, 1958; Aboussouan, 1975), photophores (Ahlstrom and Counts, 1958; Moser and Ahlstrom, 1970, 1972, 1974; Ahlstrom, 1975), myomere counts (Delsman, 1923; Dakin and Colefax, 1934, 1940; Castle, 1968, 1970; Baker, 1972, 1973; Houde, 1975; Smith, 1975), eye shape and presence of stalks (Moser and Ahlstrom, 1974; Ahlstrom, 1975), and arrangement of pigmentation (Balinsky; 1948; Winn and Miller, 1954; Castle, 1968; Leonard, 1971; Potthoff, 1975). I have mentioned the above because the authors have isolated specific characters as being either important or essential in describing certain species. It is more usual for larval forms to be described using a wide range of variables each of which on its own would not suffice. In fact any characteristics that bind one stage to another or to the adult can be used. Ahlstrom (1975) gives four main categories under which these characteristics may be grouped.

Morphometrics - Changes in body proportions as determined by measurements of body parts over a size range of specimens.

Meristics - Countable structures such as myomeres, vertebrae, rays, spines, branchiostegals, gillrakers etc.

Pigmentation - Patterns of pigment cells at different stages of larval development.

Specialized larval characters - These include features unique to a larval stage such as spines, narrowed eyes, stalked eyes, voluminous fin folds, extended snout, trailing gut, tentacles and sagittal crests etc.

The literature devoted to larval descriptions is enormous and, therefore, only a few of the more informative accounts will be listed here, in addition to those previously mentioned in this larval section: they include Fish (1932), Battle (1944), Deubler (1958), Swarup (1958), Berry (1959), Dovel (1960, 1963), Norden (1961), Mansueti (1962, 1964), Mansueti A. J. (1963, 1964), Orton (1955, 1963), Ryland (1966), New (1966), Mansueti and Hardy (1967, and references therein), Moser (1967), Benzie (1968b, 1968c, 1968d), Castle (1969: 81, list of publications), Aprieto (1974), Miller and sumida (1974) and Berrien (1975). 
As larvae grow there is often a shift in importance of diagnostic features. During the early stages the relative size and shape, myotome counts and pigmentation take priority, but as development advances more stable structures such as vertebrae, rays, spines and early scales acquire importance.

In this study each developmental series was constructed using the second method described above, where specimens were collected from the plankton and linked with other stages and with the adult. The problems peculiar to the tripterygiids, clinids and gobiesocids are discussed later (larval discussion).

The literature on the development of New Zealand marine and freshwater teleosts, formerly scattered, is now contained within a bibliography in the appendix.

\section{$\underline{\text { Terminology }}$}

Several attempts have been made to stabilize the terminology used to define stages of fish development (Hubbs, 1943, 1958; Rass, 1946; Balinsky, 1948; Jones, 1950, 1967; Lagler, 1964; Ryland, 1966; Mansueti and Hardy, 1967). However no single approach is satisfactory for all groups because of the general variations in metamoxphosis. Specific terms that are universally accepted have been applied to stages unique to groups of fish, such as leptocephali (eels, tarpons and bonefishes) and alevins (salmon and trout). The overall terminology satisfying the present study is that used by Mansueti and Hardy (1967), which is adapted from Hubbs (1943, 1958).

Yolk-sac larva or prolarva; This stage begins at hatching and continues until the yolk-sac is no longer visible.

Larva: This begins with the loss of the yolk sac, and ends with the acquisition of the adult fin ray complement. The rays need not be fully ossified.

Prejuvenile: This includes "...the intermediate period between the larval and typically juvenile form of any species in which it occurs" (Mansueti and Hardy, 1967). This period includes the differentiation of the rays, the development of juvenile pigmentation and the inception of scale formation. The tripterygiids, clinids and gobiesocids are all pelagic during this 
phase: the onset of the juvenile stage being marked by a change to a sedentary existence.

Juvenile: This stage is reached when the body shape is very similar to that of the adult and continues to when sexual maturity is attained.

Length is not an absolute indication of the age or degree of development of a larva, because there is variability in growth rate potential of given localities (Balinsky, 1948; Johnson and Barnett, 1975). More definite characteristics such as shape, ossifications, organ structure and amount of pigmentation are needed to define a particular stage. These stages or degrees of development lie within the categories of prolarva, larva and prejuvenile and relate to metamorphic sequences peculiar to a family or group of fishes. Balinsky (1948) lists 46 stages of the embryological and larval development of the family Cyprinidae based on morphological and anatomical characters. Similarly shelbourne (in Ryland, 1966) establishes stages and subdivisions for plaice larvae which relate to the migration of the eye from one side to the other. The many fin rays and spines of the tripterygiids and clinids are ossified sequentially during growth and offer a "sliding scale" which registers the degree or level of development of a particular specimen (see discussion about larval osteology). Tripterygiid and clinid larvae of different species may thereby be compared throughout metamorphosis without establishing a rigid system of stages. An excellent paper by Balon (1975) defines very clearly developmental stages and dispenses with terms such as prelarva, postlarva and prejuvenile on the grounds that they "...lack elementary logic." If terms like 'postlarva' are used then, in keeping with this, the adult fish should be called a 'postjuvenile'. Balon introduces four general terms to define the intervals of development: period, phase, step and stage. "Period is the longest interval, divided into phases, and each phase subsequently into several steps. Stage refers explicitly to a just observed, immediate moment of development" (Balon, 1975). The periods and phases given by Balon and which are applicable to the fish in this study are as follows. 


\section{Period}

$\begin{array}{lll}\text { Embryonic } & \begin{array}{l}\text { cleavage } \\ \text { embryonic } \\ \text { eleutheroembryonic } \\ \text { protopterygiolarva } \\ \text { pterygiolarva }\end{array} & \begin{array}{l}\text { embryonic } \\ \text { embryonic } \\ \text { prolarva }\end{array} \\ & & \text { larva } \\ \text { Larval } & - & \text { larva } \\ \text { Juvenile } & - & \text { prejuvenile } \\ \text { Adult } & - & \text { juvenile } \\ \text { Senescent } & & \text { adult }\end{array}$

The terms in the right hand column are those used in the present study. Balon has no direct equivalent for prejuvenile (Mansueti and Haxdy, 1967). He prefers to consider the juvenile period as beginning when the fins are fully differentiated and in so doing encompasses the prejuveniles as described here. In the tripterygiids, clinids and gobiesocids, however, there is a definite 'phase', following full differentiation of the fins. During this time the young fish are planktonic and further differentiation of pigmentation and scales etc., takes place. The end of this "phase" is marked by a change to a sedentary existence. A separate term needs to be added to the phases given by Balon to describe this interval of development. Information presented by Balon appeared too late for complete integration into this study and therefore the terminology of Mansueti and Haxdy (1967) is retained.

Body measurements were taken from larvae and prejuveniles at various stages of development. These included standard length, total length, eye length, head length, snout to vent, greatest depth, caudal depth, head width, head depth and sucker length (gobiesocids only). The number of specimens of each species measured is recorded in the larval diagnosis which precedes the larval descriptions. Only the meristic counts and measurements valuable for species diagnosis are listed or graphed at the end of the descriptions (see appendix). Terminology, as applied to the measurements and anatomical features of the laxvae, follows that of Mansueti and Hardy (1967) [adapted for larval studies from Hubbs and Lagler (1958)]. Measurements 
relating to prejuveniles are the same as for the adults, and are as described by Hubbs and Lagler (1958). Each sexies is preceded by a summary of the adult features which are important for the identification of the larvae and prejuveniles. The adult summaries for the tripterygiid and clinid species are compiled from specimens collected by the author and from relevant descriptions in the literature listed in the taxonomic review. In some cases counts from prejuveniles and juveniles are included in the sumnaries where this information defines more precisely the range of a meristic value. Summaries for the gobiesocid species are taken from Briggs (1955). Each adult diagnosis is followed by a brief outline of characteristics peculiar to the larval species. The larvae of undescribed adults are simply listed as species A, B, etc. They are, however, included in the genera to which they seem related. The larvae of Forsterygion varium are described and figured in detail țo outline basic anatomical terminology.

The duration of spawning for each species is given as determined by the presence or absence of prolarvae and early larvae in the fortnightly plankton samples (see appendix III). It was not possible to accurately establish the peak spawning times because the numbers in the samples were inconsistent. The spawning months of species whose early stages are difficult to distingish e.g. Forsterygion varium and $\mathrm{F}$. nigripenne vere obtained by noting the presence of egg clusters on the shore. The larvae and prejuveniles of all species are present in the plankton for up to 3 months after the end of spawning. Some of the series are incomplete, either because samples were inadequate or there was difficulty in diffexentiating larvae, and in particular the early stages, of closely related species.

Because of the subjectiveness of terms such as "small", "tiny" or "large", I have constructed a scale of chromatophore size. The size classes I have chosen naturally suit the larvae of this study and may have limited application elsewhere. Although the relative size of a chromatophore is readily observed on an accurate drawing, it is sometimes necessary to describe chromatophores which are not easily shown diagramatically. The scale is as follows and is applicable to expanded chromatophores of any colour.

$$
\begin{array}{rlr}
\text { (i) Tiny } & 0 & -0.05 \mathrm{~mm} \\
\text { (ii) Small } & 0.05-0.15 \mathrm{~mm} \\
\text { (iii) Medium } & 0.15-0.40 \mathrm{~mm}
\end{array}
$$$$
\text { (iv) Large }
$$$$
\text { (v) Very large }
$$$$
0.40-1.00 \mathrm{~mm}
$$$$
1.00 \mathrm{~mm} \rightarrow
$$

In this study "pigment cell" is used as a synonym of melanophore to reduce the repetitious use of the latter term. 
Adults of various species that were required for meristic data were sampled in a variety of ways. The common inshore tripterygiids were caught in mussel-baited wixe traps lowered from rocks and wharves to the bottom. The less common tripterygiids and "trap-shy" clinids were collected while scuba diving using a so-called "slurp-gun". This consists of a clear perspex cylinder (45 cm x $10 \mathrm{~cm}$ internal diameter) blocked at one end by a transparent plate which has a hole, in which another perspex tube ( $30 \mathrm{~cm} \times 5 \mathrm{~cm}$ internal diameter) is glued. Attached to the outside of the large cylinder is a stout handle, used for bracing the apparatus when in use. A circular flat plunger of laminated perspex with a handlegrip on one side slides neatly within the larger cylinder. The "gun" is aimed at a small fish and the plunger is rapidy pulled back sucking the animal in. The fish is transferred to a plastic bag by reversing the procedure. A more complicated spring loaded plunger and trigger mechanism may be fitted. The common gobjesocids were obtained from beneath stones at low tide, on semi-exposed rocky shores. The weed dwelling gobiesocids including tripterygiids and clinids were extracted from their habitat by shaking large fronds of seaweed over buckets, into which they were dislodged.

Three main methods were used to collect larvae from the plankton:

\section{(i) plankton nets. Tows with plankton nets were needed primaxily to} collect many different larvae for taxonomic work and therefore an extensive quantitative programme was not required. Nevertheless certain partially quantitative data was necessary to establish the extent of spawning times for different species. I requixed regular collections at different depths and a net that sampled efficiently.

The net used fulfilled criteria set by Smith et al., (1968) so that a filtering efficiency of $85 \%$ be maintained throughout the tow. The design was of a cylinder cone type that had a ratio of filtering area to mouth area (R) considerably in excess of 3.2, an important constant established by Smith et al., (1968). The diameter of the mouth of the net was $0.57 \mathrm{~m}$ as recommended by Matsumoto et al., (1966).

Saville (1959) demonstrated that the maximal cross sectional diameter of an organism must be greater than the diagonal of the mesh if it is to be retained. The smallest cxoss-sectional diametex of the types of larvae I 
wished to capture was close to 600 microns, so I chose a 300 micron mesh size with a diagonal length of no greater than 400 microns.

The volume of water to be filtered was set at $250 \mathrm{~m}^{3}$ enough to adequately cope with a 5 minute tow at a speed of between 1 and 3 knots. The necessary data was applied to the "greenwater" formula of Smith et al. (1968) that resulted ultimately in the construction of a net approximately $3.4 \mathrm{~m}$ long (cylinder $173 \mathrm{~cm}$, cone height $164 \mathrm{~cm}$ ). The bridle was fixed to two points high on the hoop and a flat metal depressor was attached to the lower mid-point (Matsumoto et al., 1966). This left the mouth of the net free of obstruction.

Collection, using nets, were in the main restricted to an area at Island Bay directly offshore from the Marine Laboratory. Two transects were used each one parallel to the shore, one approximately 100 metres and the other 200 metres from the outer rock line.

Ten tows were made during each day of sampling, divided between the transects in the following manner.

Transect I (i) Two 5 minute tows at 5 metres
(ii) " " " " " 10
Transect II
$\begin{array}{ll}\text { (i) Two } 5 \text { minute tows at } 5 \text { metres } \\ \text { (ii) " " " " " " " }\end{array}$

The tows each time were made in the one direction to help eliminate the effects of tidal flow. The depth at which the net operated was calculated using the angle of the warp and the amount of cable under the water. The speed of the boat was kept constant for each tow. It was not always possible to sample every two weeks as planned because of students requiring the research vessel "Tirohia" for other purposes and bad weather. A second boat a 14 foot "whaler" was used at times when the main vessel was unavailable. Uniformity in sampling was maintained by letting out the same amount of cable (for any one depth required), as used on the "Tirohia", and then adjusting the speed of the "whaler" until the required warp angle was attained. Some trips were cut short by the development of gale force winds a not uncommon event around Wellington. Sampling was further hampered by the abundance of salps during the middle sumer months. This made at times plankton towing impossible, due to clogging.

The collection programme spanned the spawning months from May 1971 
to March 1972. Because of the rocky nature of the coast all trips were made during the day.

Other plankton tows were made in Port Nicholson harbour and around the Wellington coast, whenever time and weather permitted. Tows were limited to waters less than 300 metres from the shore as these were the areas of greatest abundance of larval tripterygiids etc.

Light trapping. The light-trap as described by Ruck (1975 in appendix) was used only from wharves and boats in sheltered areas. Sampling efficiency was influenced by the weather and because of this trips were made irregularly during the spawning months. The light trap was most useful for catching late larvae and prejuveniles, that are infrequently caught in plankton nets. The apparatus was most effective after 9.30 p.m. but was of little value in areas of surge.

Light pole and dipnetting. Many of the species described in this paper, spend much of their later planktonic life within large surge channels. The larvae and prejuveniles congregate in large schools numbering several thousand and usually composed of one or two species. The surge channels are inaccessible for plankton towing and are frequently too rough for fixed light-traps. A small sealed jar was fastened to the end of a long flexible pole ( 3 metres). Within this jar was placed a $12 \mathrm{v} 6 \mathrm{w}$ bulb and holder, which was supplied with power by two wires that perforated the top of the jar and passed back along the pole to connect with a $12 \mathrm{v}$ motorcycle battery. Enough extra wire was allowed between the pole and the battery to give freedom of movement to the light pole. Two "crocodile-clips" were attached to the free ends of the wires making detachment from the battery easy. In addition an extendable pole was made wi.th a small net $(25 \times 12 \mathrm{~cm})$ at one end. The complete outfit was light and easily carried over rocks at night. The light at the end of the pole was simply dipped into the water of the surge channel. Within a short time small animals including fish larvae circled the light and were captured using the dipnet. From the net they were transferred to an adjacent container. The apparatus is most effective in calm water but may still be used in a gentle surge. Larvae and prejuveniles collected early in the study were placed in 5\% formalin, but those caught later were preserved in 5\% 'buffered' formalin. Drawings of larvae (with the help of a camera lucida) were made from live specimens or ones recently preserved. Those drawn alive were anaesthetized with Sandoz MS.222 crystals dissolved in seawater. 
Except where otherwise stated, measurements using an ocular micrometer are of specimens preserved in 5\% formalin and therefore minor inaccuracy may have resulted due to shrinkage. When it was necessary to supplement descriptions and drawings with preserved specimens, these were cleared in 50:50 glycerine and water, a dilute trypsin solution at $37^{\circ} \mathrm{C}$ or $3 \% \mathrm{KOH}$. The trypsin solution contained the following, $150 \mathrm{mls}$ of saturated sodium borate (borax), $350 \mathrm{mls}$ of distilled water and $\frac{1}{2} \mathrm{gm}$ of pancreatic trypsin.

Fin ray and vertebral counts were taken from specimens stained by Alizarin Red $\mathrm{S}$. These were prepared using a trypsin method modified from Taylor (1967) by Dr P.H.J. Castle (pers. Comm.) and a KOH technique as described by Evans (1948). Alizarin specimens were stored in 50:50 glycexine and distilled water.

The counting of myomeres was assisted by a trichrome cytology stain. The specimen is placed in distilled water and a drop of trichrome is placed on it. The trichrome stain is temporarily absorbed by the muscles making them opaque. The specimen is illuminated by incident light which highlights the contoured relief of each myomere. 
Forsterygion varium (Forster, in Bloch and Schneider, 1801)

\section{Adult diagnosis: (10 specimens)}

Maximum S.L. 100mm; D. VI-VII, XXIII-XXV, 14-15; A.II, 26-30; C. 12-14; $\mathrm{P}_{I}$. 18-21; vertebrae 48-51; nasal tentacles 5-6, orbital. tentacles $3-6 ;$ mottled brown head pigmentation and lateral U-shaped body
patterns.

\section{Larval diagnosis: (28 specimens)}

48-51 myomeres (15-18 pre-anal); 10.5m S.L. notochord upturned; $11.5 \mathrm{~mm} \mathrm{S.L.} \mathrm{sometimes} \mathrm{one} \mathrm{melanophore} \mathrm{above} \mathrm{myelencephalon;} 27$. Omm S.L. two melanophores above mesencephalon, one above telencephalon; prejuvenile at about $24 \mathrm{~mm}$ S.L.; meristic counts within range of adults; 16 pre-anal vertebrae.

Spawning months: early June to late November.

The inshore plankton is dominated by this characteristic larval form from June to December. The larvae of some species of Forsterygion appear identical and are impossible to separate with present information. A myomere count of 48-51 distinguishes the early larvae of $\underline{F}$. varium from related species which have myomere counts less than 47 . The egg and prolarval development is described by Ruck (in press a).

Larva 11.5m s.1. (Fig.1, no's 1 \& 1a). The body is long and thin with a slight taper to the tail. The organs and muscle tissues are transparent and notably without much pigmentation. The mesencephalon is relatively large at this stage, possibly due to the importance of the eyes assisting in food capture during the planktonic phase. The heart lies within the isthmus between the opercular flaps just forward of the liver. The red blood corpuscles within the heart outline the various chambers.

The overall blood circulation follows that of the prolarva (Ruck, in press a). The cartilagenous otic capsules lie posterior to the eyes and lateral to the myelencephalon.

Tubular tracts for the three semi-circular canals are visible particularly fxom the dorsal aspect (Fig.l, no.la). Two otoliths lie within each capsule and the capsules bulge laterally beyond the surface of the body muscle while the postero-medial surface receives the fibres of the first myomere of the body. The medial surface of each capsule is moulded to fit neatly about the hindbrain while the anterior inner face is contoured around the posterior curvatuxe of the midbrain and continues forward 
around much of the brain as the cartilagenous neurocranium. The olfactory structures are present as shallow cups with lateral edges beginning to curl inward. The eyes lie within laterally expanded transpaxent domes of epidermal tissue, the size being accentuated by immersion in $5 \%$ formalin. Dorsally the pigmented outline of each eye is subtriangular with the apex pointing under the brain. The spherical lenses protrude laterally and are covered by transparent corneal tissue. The chorioid is black, in contrast to the silvery-blue iris about the lenses. Delicate jaw elements surround the mouth forming a functional suctorial feeding mechanism. The gut is long and convoluted lying partially sandwiched between the two trunk muscle flaps. Just behind the liver the gut is slightly bunched in anticipation of future coiling. A flattened air bladder is present above this bunched area. The pectoral fin is a transparent flap of tissue with an expanded base attached to the cleithrum. Each cleithrum is visible as a refractive line running obliquely fxom the ventral midline to the second myomere. The front of the notochord angles down beneath the hindbrain while the terminal tapered end angles upward in the tail as the urostyle. The dorsal and ventral fin fold are tattered, possibly due to continuous use or the pressures they are subjected to in the plankton net. Ten weakly ossified (at the base only) caudal rays extend from the hypural plates. No other rays or spines are present in any form at this stage. A small melanophore is situated in the parietal peritoneum of the abdominal cavity in front of the liver. Two large melanophores lie in the upper parietal peritoneum, one above the air bladder and the other just forward of the anus. A series of 17-27 small melanophores run along the ventral midine of the tail. These in later larvae form a series which correspond roughly to the number of rays in the anal fin. In the early larvae the number of melanophores is variable and is therefore a poor diagnostic feature. In some individuals a medium sized melanophore is situated within the neural tube above the narrowest portion of the medulla oblongata. The myomeres have a double chevron shape.

Larva 14.9mm s.1. (Fig.1, no.2). The head appears more robust, having increased in depth and width. The edges of the olfactory cups have curled inward to meet almost at the mid-line, forming a tube like olfactory capsule. The preoperculum is well defined and the branchiostegals are faintly visible, protruding from beneath the opercular series. The rows of 
gills are also visible beneath the operculum. The erythrocytes percolating through the lamellae impart a red tinge to the gill series. At the lower end of the cleithra are two pelvic fin buds. They arise in the jugular position characteristic of the adult, therefore there is no pelvic migration during metamorphosis. The liver is displaced to the left of the midline and is barely visible on the right side of the larva. The gut forms a single right hand coil effectively shortening and deepening the body cavity. The anus is drawn closer to the head, but the preanal myomere count of 17-18 is unchanged. Coiling obscures the slender air bladder.

Each centra takes up alizarin (hence is ossified) at this stage although the intensity of staining of the whole column, including many elements of the skull, decreases toward the tail. In contrast the first rays to ossify are those of the caudal fin followed by the thixd dorsal and anal fins, progressively moving toward the head. In any one fin the rays or spines which grow the largest in the adult are the first to ossify and hence stain more deeply than tl rest. In the third dorsal fin, for example, the central rays absorb the most stain with a gradual decrease in the rays anterior and posterior to these. The caudal rays have up to 3 segments ossified, as well as 3 dorsal and 3 ventral procurrent rays. The lower portions of 15 third dorsal rays are stained lightly, likewise 29 anal rays. There are no spines in the first and second dorsal fins. The urostyle curves steeply upwards and extends into the caudal fin. With the full caudal ray complement present and the hypural elements pointing rearwards the caudal $f$ in is much like that of the adult. A fuller study of the general osteological development of $F$. varium is included earlier in this thesis.

The angle of the myomere chevrons increases posteriorly. Pigmentation pattern is unchanged, apart from an increase in the ventral melanophores associated with the anal rays $(25-27$ plus 3 ventral to the peduncle). These may form a continuous series ( 1 per segment) or one with several gaps (i.e. several segments missing). Melanophores about the brain and spinal cord are generally absent up to about $16 \mathrm{~mm}$ s.1. However some individuals possess a medium sized melanophore above each lobe of the mesencephalon, one above the medulla and a series (up to 15) along the spinal cord below the third doxsal fin. Those with well defined neural melanophores also have a well developed continuous anal and caudal series and several melanophores along the base of the third 
dorsal fin, like those found more consistently in later stages. Apparently these larvae have the potential to produce a specific complement of pigment cells which may not show because of either developmental processes, the effects of fixing or exposure to intense (microscope) light in the case of live specimens.

Larva 22.2m s.1. (Fig.1, no's 3, 3a \& 3b). The larva is proportionately the same as at $14.9 \mathrm{~mm} \mathrm{~s} .1$. with the greatest depth beneath the third dorsal fin. All the rays of the third dorsal and anal fins are distinct and have faint signs of segmentation. The two spines of the anal fin and those of the second dorsal are also well defined. The spines of the second dorsal are longest and best ossified at the posterior end, sloping gently anteriorly. In the adult, however, the longest spines of the second dorsal fin are more anterior. The trend of gradual ray and spine development from the back to the front of the larva, has (because of the length of the second dorsal) superseded the tendency for the longest elements of individual fins to ossify first. In contrast, the longest adult spines of the typically short first dorsal fin are the first to ossify in later larvae, especially in species e.g. Notoclinus compressus where there is a marked difference in spine length. The rays of the caudal fin are well segmented. Centrally above the telencephalon is a medium sized melanophore attached to the inner surface of the neurocranium. Those above the mesencephalon and myelencephalon ace present in all specimens of this size. The brain is also surrounded by several tiny and spindly xanthophores which produce a yellow corona around the brain. Approximately 24 medium sized stellate melanophores cover the spinal cord as an interdigitated series running from above the anus to below the last ray of the third dorsal fin. When expanded the neural melanophores shroud much of the brain and spinal cord in black. In larval tripterygiids etc., the central nervous system is one of the first organs covered by pigment cells. This suggests a function related to the nervous tissue itself rather than a need for cryptic colouration. It seems the larvae may need to control the amount of light reaching the nervous tissue at some critical stage in their development. Light, especially visible light is most injurious when absorbed (Eriedxich, 1961; Eisler, 1961). Earlier larval stages without neural pigmentation may be protected from radiation by their transparency (Breder, 1962) because light passes through 
them and is not absorbed. As the larvae grow larger, however, the absorptive property of the body tissues, in particular the nervous system, may alter, necessitating protective pigmentation. The uppermost surfaces are pigmented first as the greatest light intensity for pelagic forms is from above, Seventeen melanophores run along the dorsal mid-line, at the base of the rays in the third dorsal fin. Three of these lie separately above the caudal peduncle. At the base of the caudal rays are 3 small melanophores interspersed by 4 spindly erythrophores (Fig.1, no.3b). Two medium sized melanophores are placed at the base of the pelvic buds along with a small one on the isthmus (Fig.1, no.3a). Within the branchial chamber are 3 large stellate melanophores, two on the preoperculurn and one on the cleithrum. Hazy melanic pigmentation is present on the maxillary and dentary. The liver is large and overlaps the coil in the gut.

At approximately $20 \mathrm{~mm} \mathrm{s.1.} \mathrm{the} \mathrm{larvae} \mathrm{of} \mathrm{E.} \mathrm{varium,} \mathrm{along} \mathrm{with}$ those of related species, leave the offshore plankton and form schools each of several thousand individuals in the surge channels. The larvae school just below the surface in the middle of the channels away from surrounding rocks and weed and move with the tidal surges. Individuals maintain position by heading into the current. The larger and more heavily pigmented prejuveniles are cumbersome swimmers and assume an oblique jerky attitude similar to that of the adults. This occurs just prior to settling, when the prejuveniles become relatively sedentary and probably results from an increase in density producing a decrease in buoyancy. Each school reacts spontaneously to localized disturbances by diving deeper or evading rapidly. This migration from deeper waters partly explains the absence of these (20mm larvae) and largex larvae from plankton tow samples taken offshore.

Prejuvenile 27mm s.1. (Fig.2, no's $2 \& 2 a$ ). With an increase in the ossification of structures in the head, individual elements of the jaws, suspension and opercular series have become visible. The olfactory capsule is closely associated with the suspensory elements. The brain appears longitudinally compressed and compact with the tip of the telencephalon no further forward than the eyes. Each lobe of the brain is precisely defined particularly on live specimens. The integumentary and muscle layers become clouded when fixed in solutions of formalin. The mesencephalon is expanded laterally pressing against the inner surfaces of the olfactory capsules. The median metencephalon projects upwards 
between the anterior lobes of the myelencephalon. The overall pigmentation is the same as for the $22.2 \mathrm{mrn}$ larva although individuals show variation in the number of melanophores along the dorsal mid-line and the spinal cord. The spinal pigment forms a series of up to 24 separate cells, similarly for the dorsal fin having a possibility of one melanophore per ray, and 2-3 above the peduncle. Each fin has the full spine and ray complement characteristic of the adult but not fully ossified. The spines of the first and second dorsal fin lie low to the body. The first spines of the second dorsal are the longest now, sloping gradually postexiorly. The pectoral rays are segmented but unbranched with the lower ones thickened slightly.

Prejuvenile 28.7 mm s.l. (Fig.3 \& Fig.4). Only one specimen was caught in the light-trap. This specimen was apparently planktonic and lies on the hazy line between prejuvenile and juvenile. The typical tripterygiid fin pattern is demonstrated and the pigmentation while extensive is incomplete. The profile of the head is low and rounded and the eyes do not break the contour. In contrast, that of the adult is steep with the eyes interrupting the contour. The anterior nostril is tubular with a single spatulate tentacle on the posterolateral edge. The snout is rounded and the mandibles fleshy passing obliquely down to terminate beneath the anterior margin of the pupil. Directly above each eye is a divided integumentary tentacle. The opercular flap ends beneath the last ray of the first dorsal fin. The pelvic fins, each consisting of two main rays, emerge forward of the pectoral base and pass ventrolaterally. The pectoral fins are large with narrow bases and broad webs. The rays form a graded series from the delicate, straight and unbranched upper ones to the curved, thickened lower ones. The lower rays of the bottom dweliing txipterygiids are typically thickened, presumably in response to contact between the pectoral fins and the substrate. The dorsal and ventral. profiles (Fig.3) are essentially the same as in the adult. The first dorsal is rounded with the first and last spines the shortest. The web of the first dorsal fin is paxtly attached to the second dorsal as is the second to the third dorsal fin. Similar to the adult, the second dorsal is higher than the first and the sixth spine is the longest with a gradual reduction in spine size anteriorly and posteriorly. The third dorsal fin is of equal height to the second, and has rays which decrease 
in size posteriorly. The tail is slightly notched unlike the rounded condition of the adult.

An overall red-orange colour is present in the dorsal, caudal and anal fins and the dorsal half of the trunk and peduncle. This is due to a combination effect of melanophores and erythrophores in the integument. Excluding the head, pigmentation on the ventral surface is absent, with the exception of the melanophores associated with the rays of the anal fin. The dorsolateral pigmentation of the trunk and peduncle consists of a series of U-shaped markings (5) which begin just above the mid-line and extend up into the dorsal fins as oblique bars. These markings blend into each other laterally, particularly just behind the head giving an irregular mottled effect. The oblique extensions of the markings, leading into the dorsal fins are composed predominantly of erythrophores and are therefore more red than the patches on the body. The oblique bars of the second dorsal fin run together to give a red fringe to the fin. In contrast the upper fringe of the first dorsal is black due to a predominance of melanophores. The pectoral fins have a red tinge from the numerous tiny erythrophores lying on and between the rays. Along the lateral aspect of the body are distinct pigment patches (equal numbers of melanophores and erythrophores) running from the base of the pectoral fin to the caudal base. Anteriorly they appear as attenuated inverted U's while posteriorly they are almost circular and blend in with the dorsal markings. The overall markings are identical on both sides of the fish. The integument of the head is extensively covered with pigment cells both dorsally and ventrally. The arrangement is best observed in Figure 4. The 3 basic melanophores are still visible above the brain lobes. Most of the melanophores and erythrophores around the brain are situated on the inside of the neurocranium not on the superficial integument. Beneath the eyes there is a disrupted bar pattern extending over the opercular series and onto the base of the pectoral fin. This pattern is not yet interspersed with the yellow and green pigment which gives the "tiger" pattern in the adults. The iris is also irregularly pigmented. 
Forsterygion nigripenne (Valenciennes in Cuvier and

Valenciennes, 1836)

Adult diagnosis: (35 specimens)

Maximum S.L. 90mm; D. V-VII, IXX-XXII, 12-15; A. I-II, 25-27;

C. 12-14; $\mathrm{P}_{I}$ 17-19; vertebrae 43-47; nasal tentacles 1-3; orbital

tentacles $1-4$; lateral line I 16-20.

Larval diagnosis: (26 specimens)

45-47 myomeres (marine form) (15-17 pre-anal); $9.25 \mathrm{~mm}$ S.L. notochord upturned; $9.8 \mathrm{~mm}$ S.L. one melanophore above myelencephalon; 26. Omm S.L. 4-5 melanophores above mesencephalon, one above telencephalon; prejuvenile at about $20 \mathrm{~mm}$ S.L.; meristic counts within range of adults; 15 pre-anal vertebrae.

Spawning months: July to October.

The main morphological differences between the extreme forms of F. nigripenne do not show during the larval development. There are however subtle differences in the anal, third dorsal ray and vertebral counts which are manifest in the prejuveniles. These differences are as follows:

Estuarine form

Third dorsal

Anal spines and rays

Vertebrae
$13(12-14)$

II 25 (II, 24-26)

$44(43-45)$
Sheltered marine form

$14(13-15)$

I 26 (I-II 25-27)

$46(45-47)$

The identification of F. nigripenne larvae duxing the very early stages relies on myornere counts, size, shape and locality. The initial development is virtually identical, in terms of size and shape, to that of $\mathrm{F}$. varium except that the larvae of $\mathrm{F}$. nigripenne have fewer myomere segments (43-47 cf. 48-52). The myomere count (= vertebrae) is however insufficient to separate them from several undescribed forms such as the "yellow and black blenny" "oblique-swimming blenny" and Forsterygion capito. The latter species of tripterygiids, which are relatively readily distinguished as adults have overlapping meristic values, which makes the identification of earlier stages extremely difficult. Nevertheless they are small as adults and the size of their larvae at different stages of development is less than that of equivalent stages of $\mathrm{F}$. nigripenne. The larvae described in this series were taken from within wellington Harvour, well away from strictly estuarine conditions. The vertebral 
range (43-45) of the estuarine form of $\mathrm{F}$. nigripenne overlaps with that of $\mathrm{F}$. capito etc. $(42-44)$, more than the marine form of F. nigripenne (45-47). Therefore, for positive identification attention was given to larvae with myomere counts between 45 and 47 .

Larva 9.8mm s.1. (Fig.5, no's 1 \& la). The overall pigmentation is essentially the same as for F. varium, except the medullary melanophore is present in most individuals of this size. Fifteen to 20 melanophores run along the ventral midline of the tail. The presence of numerous poorly defined xanthophores impart a yellow tinge to the tissues about the brain and above the gut. Seven to 8 weakly ossified rays are present in the caudal fin. Ten are visible at 11-12m s.1. in keeping with a similar development of $\mathrm{F}$. varium. The larvae of $\mathrm{F}$. nigripenne have 15-17 preanal myomeres compared with $16-18$ in $\mathrm{F}$. varium.

Larva $12.2 \mathrm{~mm} \mathrm{S.1.} \mathrm{The} \mathrm{gut} \mathrm{has} \mathrm{a} \mathrm{right} \mathrm{hand} \mathrm{coil} \mathrm{directly} \mathrm{beneath}$ the anterior peritoneal pigment patch. The xanthophores in some areas appear as distinct stellate cells: two dorsally between the optic lobes and the cerebellum and 3 in the upper parietal peritoneum of the gut between the melanic patches. Several smaller less defined xanthophores surround the brain as well as the notochord directly beneath the third dorsal fin. Approximately 20 inid-ventral melanophores are present plus two beneath the caudal peduncle. The urostyle j.s fully upturned with the hypurals directed rearwards and supporting 13 to 14 well defined, but incompletely ossified rays. Because the middle rays are the first formed and the longest, the posterior edge of the caudal fin is rounded. In later larval stages the upper and lower rays grow longer to produce a notched caudal edge. This notched condition is not maintained in the adults which have rounded or straight caudal edges. Some of the rays of the third dorsal and anal fins are visible as tiny strips of granular tissue running about $0.2-0.3 \mathrm{~mm}$ into the finfolds.

Larva 16.5m s.1. (Fig.5, no.2; Fig.6, no.1). The overall shape is the same as in equivalent stages of F. varium (1.5-1.6m s.1.) but the onset of pigmentation occurs slightly earlier. There are 6 medium sized melanophores along the dorsal midline associated with the rays of the third dorsal fin, two above and below the caudal peduncle and two at the base of the caudal rays. In most individuals the melanophores on the ventral midline form a continuous series ( 24 to 25 ) associated with 
the anal rays. The medullary melanophore is large although the visible size may vary according to degree of expansion. More xanthophores are individually defined particularly adjacent to the otic capsules, above the pineal stalk (shown black Fig. 5, no.2) and around the notochord. Some specimens have signs of melanophore formation above the posterior portion of the spinal cord. The trend of ossification of rays and spines is identical to that of $\mathrm{F}$. varium.

Prejuvenile 26m s.1. (Fig.5, no.3; Fig.6, no's $2 \& 2 a$ ). It is during the later stages of development that the larvae and prejuveniles

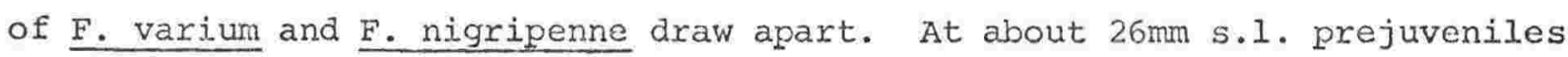
of $\mathrm{F}$. nigripenne are stocky and have the full spine and ray compliment of the adult. Equivalent sizes of $\mathrm{F}$. varium have no first dorsal fin developed and appear more slender. The bony elements of the suspensory system are well defined beneath the transparent integumentary layers (fresh or living specimens only). The lips are fleshy. Each nasal tube is U-shaped, and lies close to the eyes, forming the anterior wall of the orbit. The anterior nostril is bordered posteromedially by a small integumentary flap, precursor of the denticulate tentacles of the adult. The anterior dorsal tip of the cerebral hemispheres finish level with the anterior edge of the pupil, but continue ventrally beneath the nasal tubes as the olfactory tracts. The dorsal profile is essentially straight but for an upward kick in front of the third dorsal. The contour at the base of the third dorsal rays and to a lesser degree the anal fin is indented in most tripterygiid larvae. This is due to the large distal radials (of the ptexygiophores) and associated muscles protxuding above the epaxial muscle masses. The first dorsal fin is lower than the second which is in turn lower than the third. The caudal fin has the full complement of segmented and procurrent rays. The spine in front of the anal fin is shorter than the other rays. The exact size and number of melanophores above the brain is variable, although they are restricted at this stage to the mesencephalon, where there is a basic pattern of 4 . These 4 may be accompanied by several other dorsolateral melanophores. Numerous small xanthophores surround the brain (shown as faint stellate chromatophores Fig.5, no.3) and opercular region imparting a yellow corona to the organs of the head. A large medullary and an anterior spinal melanophore is present, but none occur above the telencephalon at any stage of development unlike $F$. varium. Approximately 20 melanophores continue the neural series down the spinal 
cord below the third dorsal fin. Three melanophores surround the isthmus at the base of the pelvics (Fig.6, no.2a). A large melanophore is visible on the pectoral base beneath the branchiostegal membrane. Eight, irregularly placed, eryth rophores lie beneath the gut. In front of the third dorsal fin are 8-9 melanophores that are part of a future series which spread ventrolatexally. Each ray of the third dorsal fin has a melanophore at its base. The number of melanophores on the peduncle and at the base of the caudal rays is variable, and is usually associated with erythrophores and xanthophores. Each anal ray is accompanied by a basal melanophore. The iris is silvery-blue in contrast with the rest of the eye which is black.

Prejuvenile $27.5 \mathrm{~mm} \mathrm{s.1.} \mathrm{(Fig.5,} \mathrm{no.4;} \mathrm{Fig.6,} \mathrm{no.3).} \mathrm{The} \mathrm{depth}$ has increased proportionately more than the length, giving a robust appearance. The relative shapes and sizes of the fins are the same as in the adult. All the rays are partially or completely segmented and well ossified. The profile of the head is rounded anticipating the robust head which is charactexistic of the adult. Spatulate tentacles are present on the anterior nostrils and above the eyes. The first scales to form are those above the lateral line leading back from the opercular flap. The basic pigrnentation of earlier stages is obscured by the addition of numerous melanophores over broad areas of the body. This pigmentation is rapid. It may occur in any individuals larger than $25 \mathrm{~mm} \mathrm{s.1.} \mathrm{and} \mathrm{coincides}$ with the settling out of prejuveniles from free-swimming schools. Specimens with only the neural and ray melanophores have been observed, in aquaria, to settle on the bottom and undergo pigmentation overnight. Some partially pigmented specimens will remain in the schools, and these stand out clearly from the more transparent forms. The general pattern herein described is from a specimen which is the same size but slightly more advanced in terms of pigmentation than the one figured.

The basic patterns are composed almost entirely of melanophores, with some xanthophores and erythrophores in areas previously described. The area between the eyes, the nostrils, anterolateral to the eyes, the anterior portion of the upper $\mathrm{Iip}$ and the anterior and sub-terminal portions of the mandible are relatively evenly covered by tiny stellate melanophores. The tentacles above the eyes also carry tiny melanophores. Lateral to the head (including the circumorbital region, preoperculum, operculur and otic capsule) the pigmentation is patchy making it difficult to visualize any 
specific arrangement. At its best, three bars extend from the edge of the eye (a) across the posterior portion of the maxillary and premaxillary (b) to the point of articulation of the jaw, and (c) from the posteroventral point of the eye to the preoperculum. A bar of pigment cuts anterioxly and obliquely across the otic capsule. This runs parallel with another less distinct but broader bar which crosses from the first myotome to the broad surface of the opexculum and down to the lower junction of the operculum and suboperculum. Numerous tiny scattered melanophores lie in between these bars tending to obscure the pattern. The lateral and dorsolateral interior surface of the gill chamber is covered by a broad even sheet of melanophores. Dorsally the brain is covered in medium sized melanophores lying close to the surface of the brain and quite separate from other melanophores lining the inner wall of the neurocranium. Above the optic lobes are 4 large melanophores, a carry over from earlier pigmentation. The melanophores and xanthophores on the brain are generally larger and closer together than those on the rest of the body and hence the lobes of the telencephalon and mesencephalon become clearly outlined. This is characteristic of many tripterygiid prejuveniles. A large cluster of melanophores occurs on the pectoral base. Running laterally from behind the base of the pectoral fin to the caudal rays are 9-10 H-shaped melanic patches, which with expansion of the lower arms become more like a series of inverted U-shaped patches. Along the dorsolateral aspect of the trunk and tail are two rows of poorly defined patches. In these the melanophores are concentrated on the myomeres outlining a chevron series along the body. These dorsolateral rows later become better defined. The lowest of the two becomes almost continuous as a zig-zag line. The uppermost line forms a series of small blocks running along the body and tucked into the V's of the zig-zag line. Along the dorsal mid-line are approximately 9 patches surrounding the bases of the dorsal fins. These extend obliquely forward on the fin membranes producing a barred pattern. Four vertical stripes are present on the caudal fin. The ventral surface is free of pigmentation except for the anal series. In later stages the base of the anal fin is covered in tiny melanophores leaving an unpigmented outer fringe. At $30 \mathrm{~mm}$ s.1. all specimens have settled, are fully scaled and have denticulate orbital tentacles. Individuals this size found in estuaries show signs of the large first dorsal fins which are characteristic of estuarine adults. 
Adult diagnosis: (20 specimens)

Maximum S.L. 65mm; D. VI, IXX-XXI, 11-13; A IT' 25-26; C. 12-13;

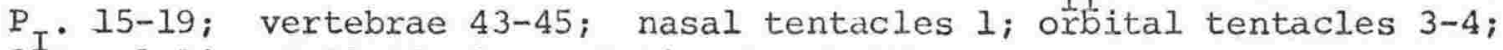
lateral line I 21-23; lateral line II 22-25.

Spawning months: July to October.

While the larval series of $\mathrm{F}$. capito cannot be defined without more complete information on related forms, the prejuveniles can be recognized using meristic counts in conjunction with peculiarity of pignentation. The basic pigmentation of late larvae is somewhat similar to $\mathrm{F}$. nigripenne with the exception of two large melanophores above the mesencephalon (c.f. 4) and a single large one above the telencephalon (c.f. none in late larvae of F. nigripenne). General pigmentation of the head, trunk and tail takes place at about 20-22 mm s.l. and is well laid down by 22-23 mm s.1. This reflects the generally smaller sizes of the larvae at particular stages of development compared with those of $\mathrm{F}$. variurn or F. nigripenne. This size shift is apparent even in the very early stages where the average standard lengths of the prolarvae of $\mathrm{F}$. varium and F. nigripenne are $5.85 \mathrm{~mm}$ and $5.95 \mathrm{~mm}$ respectively, compared with $4.9 \mathrm{~mm}$ for those of F. capito (Ruck, 1973a; in press a). The onset of pigmentation affects the same areas as F. nigripenne with an increase in the number of melanophores on the brain, head, trunk and tail. However in the prejuvenile and juvenile stages the zig-zag line darkens while the other lighter rows blend as a uniform layer over the body and fins. The brain lobes remain outlined and the barred pattern in the dorsal fins is still visible. The overall affect is a distinct dorsolateral line along the head to the caudal. fin.

Gilloblennius tripenni.s (Forster, in Bloch and Schneider, 1801)

\section{Adult diagnosis: ( 6 specimens)}

Maximum S.I. $70 \mathrm{~mm} ;$ D. III, XV-XVI, 13-16; A. II 22-24; C. 12-13; P 15-17; vertebrae 38-40; nasal tentacles 4-9; oxbital tentacles 1.5-18; lateral line scales $31-34$.

Larval diagnosis: (21 specimens)

38-40 myomeres (10-12 pre-anal); $8.2 \mathrm{~mm}$ S.L. notochord upturned; 
centrally placed melanophores above brain; at least two elongate interdigitated melanophores above caudal peduncle; prejuvenile at about $14.0 \mathrm{~mm}$ S.L.; meristic counts within range of adults; 1.0-12 pre-anal vertebrae.

Spawning months: July to October.

The embryological and early larval development is described by Ruck (in press b). The larvae of G. txipennis are less attentuated than those of Forsterygion species. The prolarvae average $5.72 \mathrm{~mm} \mathrm{s.1}$. at hatching and by $7.0 \mathrm{~mm} \mathrm{s.1.} \mathrm{the} \mathrm{gut} \mathrm{is} \mathrm{strongly} \mathrm{coiled.} \mathrm{This} \mathrm{makes} \mathrm{the}$ head region typically robust in contrast with the slender tapered tail. While the embryological development is similar to that of G. decemdigitatus (Ruck, in press a) the larval development is quite different, particularly the pigmentation.

In the plankton there are numerous larvae with superficially similar characteristics to those of G. tripennis. These will undoubtedly remain unidentified until the entire tripterygiid nomenclature is clarified. Because of these similar forms I have applied rigid criteria for larvae assigned to $\mathrm{G}$. tripennis. These criteria may not allow for all the variations inherent within this larval species but it does mean that positive identification of some individuals is possible.

Larva $7.7 \mathrm{~mm}$ s.1. (Fig. 7, no's 1, la \& 1b). The blunt head is broad and the olfactory cups are shallow and uncurled. Dorsal and ventral fin folds are continuous with the caudal lobe, and there are no rays or spines. Thexe is an aggregation of caudal ray tissue beneath the end of the straight notochord. The neural melanophores are conspicuous with a medium sized one above the mesencephalon and the myelencephalon and a small one on the spinal cord just behind the medulla. Two to 3 small melanophores are sometimes present well down the spinal cord, although these may be absent in some individuals. Earlier in development the melanic patch above the gut was divided in two but with the extreme coiling of the gut and consequent forshortening of the abdominal cavity these have come together. The pectoral flap extends two-thirds of the way along the gut. There is a small melanophore in the parietal peritoneum at the anterior ventral corner of the liver, in line with two more along the integument of the belly. Melanophores are present along the dorsal mid-line of the tail and are expressed in 3 main ways (Fig.7, no.la). All specimens have at least two elongate pigment cells which may interdigitate. Along the ventral mid-line is an irregular series of 5-11 small melanophoxes and a 
tiny one is usually present on the anterior maxgin of the caudal anlage. Approximately 40 myomeres are present, 11 (10-12) of these are pre-anal. Dorsally the brain lobes are well defined with the telencephalon more elongate than in other tripterygiids (Fig.7, no.1b).

Larva $9.3 \mathrm{~mm} \mathrm{s.1.} \mathrm{(Fig.7,} \mathrm{no's} 2 \& 2 a$ ). The depth of the tail has increased making the head appear less robust. The myomeres of the trunk have grown laterally reducing the step between the otic capsule and the first myomere. The notochoxd is upturned and continues well into the caudal lobe. Each hypural carries 4 rudimentary rays which are ossified at the base. All the vertebral centra are ossified but there are no ray or spine anlages in the dorsal and anal fin folds. The anterior neural melanophores have not changed but the posterior spinal ones have increased to 6 or 7. The melanophore above the telencephalon shows a condition frequently found in large pigment cells. Here the pigment material is present in the extremities of the cell leaving a concentrated dot at the centre surrounded by a clear ring. A small melanophore is situated on the isthmus in front of the pelvic buds and there are still. two on the belly. Melanophores along the ventral mid-line have increased to about 13 with the last 3 or 4 close together beneath the caudal peduncle. Two elongate pigment cells border the hypurals at the base of the caudal rays. An additional 2 or 3 small melanophores are present along the dorsal mid-1ine.

Prejuvenile $14.8 \mathrm{~mm}$ 5.1. (Fig.7, no's $3 \& 3 a$ ). All the fin rays and spines are visible. The larvae of $F$. varium and F. nigripenne at an equivalent size show no sign of spines in the dorsal fin and therefore their development is retarded relative to $\mathrm{G}$. tripennis. The spines of the first dorsal fin are distinctly descendant but lower than those of the second which in turn are lower than the rays of the third. All the rays of the caudal and third dorsal fin are segmented. The pectoral fin is large and spatulate extending back level with the eighth spine of the second dorsal fin. The pelvic rays are slender curved elements extending posterolatexally across the belly. Ossification is sequential following a similar pattern to that of $\underline{F}$. varium. Structural elements of the head are well defined; in particular the jaws and U-shaped nasal tubes. The anterior nostril has a small dermal flap on the posterior margin. There is also a small orbital tentacle which consists of a small mound of integument 
directly above the eye. The brain is compact and does not extend forward of the anterior margins of the eyes.

The basic arrangernent and number of pigment cells is unchanged except for an increase in the number of spinal melanophores to 10 and the appearance of a medium sized melanophore above the telencephalon on the inner wall of the neurocranium.

\section{Gilloblennius decemdigitatus (Clarke, 1879)}

Adu.lt diagnosis: (10 specimens)

Maximum S.I. $95 \mathrm{~mm} ;$ D. III, XVII-IXX, 14-16; A. I 26-29;

C. 11-13; $P_{I}$. 18; vertebrae 42-44; nasal tentacles 3--6; orbital tentacles 9-15; lateral line scales 32-35.

\section{Larval diagnosis: (21 specimens)}

42-44 myomeres (10-12 pre-anal); $9.3 \mathrm{~mm}$ S.L. notochord upturned; 4-5 elongate melanophores along dorsal and ventral mid-lines; prejuvenile at about $14 \mathrm{~mm}$ S.L.; meristic counts within range of adults; 10-12 pre-anal vertebrae.

Spawning months: Early June to early December.

The embryological and early larval development is described by Ruck (in press a). The prolarvae and larvae have a pigment pattern which sets them apart from all other known New zealand tripterygiid species. Along the dorsal and ventral mid-lines are several large and unusually elongate evenly spaced melanophores interspersed by numerous tiny erythrophores.

Larva $9.7 \mathrm{~mm}$ 5.1. (Fig.8, no's 1 \& 1a). The head tapers more evenly toward the tail than it does in G. tripennis. Rudimentary hypurals support approximately 10 caudal rays beneath the slightly upturned end of the notochord. These rays are ossified at the basal. ends. There are no spine or ray anlages in the dorsal and ventral fin folds. Some of the centra above the hind-gut are faintly ossified. The gut is coiled and there is a prominent airbladder just forward of the anus. There is a large melanophore above the optic lobes and the spinal end of the myelencephalon. The posterior of the two melanic patches present in the dorsal parietal peritoneum overlies the aixbladder. These patches have come together by the coiling of the gut and associated compression of the abdominal cavity but not to the same degree as in G. tripennis. A medium sized melanophore lies in the anteroventral corner of the gut cavity at the apex of the liver. Along the dorsal mid-line are 3-4 (usually 3) large, elongate 
melanophores regularly spaced between the anus and the caudal fin. There is a corresponding 3-4 (usually 3) pigment cells along the ventral mid-line. All of these send dendxitic extensions across the myomeres either side of the fin folds. Between these large melanophores are rows of tiny erythrophores, figured as small black dots, running each side of the dorsal and ventral fin folds. Ventrally the two rows extend forward onto the belly as far as the cleithral symphasis. There are approximately 9 exythrophores per millimeter. A single small melanophore is present between the hypural plates at the base of the caudal rays. The melanophores of the chorioid overly a green background colour, imparting a mottled green hue to the back of each eye. No spinal melanophores are visible at this stage. There are 11-12 pre-anal myomeres and a total of $42-44$.

Larva $10.6 \mathrm{~mm} \mathrm{s.1.} \mathrm{The} \mathrm{urostyle} \mathrm{is} \mathrm{fully} \mathrm{upturned} \mathrm{but} \mathrm{still} \mathrm{runs}$ into the caudal fin. Thirteen finely segmented caudal rays are visible. Pigmentation has altered little except for an additional melanophore well. forward on the spinal cord, a medium sized one on the isthmus of the throat and another small one within the pericardium either side of the heart. A small pigment cell lies on the junction between the two premaxillae.

Larva $11.4 \mathrm{~mm} \mathrm{s.1.} \mathrm{(Fig.8,} \mathrm{no's} \mathrm{2,} \mathrm{2a \&} \mathrm{2b).} \mathrm{The} \mathrm{edges} \mathrm{of} \mathrm{the}$ olfactory cups are slightly curled inward and the suspensory elements are well defined. Twelve to 13 segmented and well ossified rays are present in the caudal fin, which has a rounded posterior margin. several procurrent rays are also visible and the urostyle still penetrates slightly the caudal membrane. The future rays of the third dorsal fin are represented by 13 weakly ossified strips leading into the dorsal fin fold. Similarly there are 24 rudimentary anal rays in the ventral fin fold. There are 15 narrow divided rays in the pectoral fin, and two small pelvic ray buds extend posterolaterally from the cleithral symphasis. Several more neural melanophores have appeared, a small one above the telencephalon, two more on the anterior spinal cord (giving a total of 3 excluding the one on the medulla) and a series of 25 along the spinal cord. The latter run from above the anus to a point beneath the last ray of the third dorsal fin (Fig.8, no.2). Directly above the anus either side of the dorsal fin fold are two medium sized pigment cells. There are frequently 4 (c.f. 3 previously) melanophores along the dorsal and ventral mid-lines 
at this stage. The melanophore between the hypurals is still present as are the dorsal and ventral erythrophore series. Two rows of 5-6 small melanophores run along the belly adjacent to the closing flaps of the abdominal hypaxial muscles (Fig.8, no.2a).

Larva $13.5 \mathrm{~mm}$ s.1. (Fig.8, no.3). Apart from the first dorsal fin the full ray and spine complement is visible, and all the rays are segmented. The second dorsal fin is low and the spines are even. The spines, rays, centra, suspensory elements and some bones of the neurocranium are ossified. Each nasal tube is U-shaped and there are no nasal or orbital tentacles. Spinal melanophores have increased in number to 31-32 excluding the one above the medulla. The posterior pigment cells equate one per vertebral centra, and a medium sized melanophore sits above the urostyle. Lateral to the second and third to last centra, are two elongate melanophores which lie on the horizontal septum between the epaxial and hypaxial muscles. A further two border the hypural plates. A small melanophore sits on the upper end of each maxillary and a large one is present on the medial surface of the gill chamber (shown in a contracted state Fig.8, no.3). Pigmentation in the integument of the belly is not always present and the melanic patches above the gut are larger and confluent. The shape, size and arrangement of melanophores along the dorsal and ventral mid-lines are adequately explained by the drawing (Fig.8, no.3) and with the exception of one or two small melanophores the number is the same.

Prejuvenile $25.7 \mathrm{~mm} \mathrm{s.1.} \mathrm{(Fig.9,} \mathrm{no's} 1$ \& la, single specimen). The fin ray and spine complements and relative fin sizes are the same as those in the adult. The dorsal profile is essentially straight and the head is characteristically flattened. Each pectoral fin is large and spatulate extending back level with the second ray of the anal fin. Simple spatulate tentacles are present on the anterior and posterior nostrils and above the eyes. Three lateral line scales are formed. Pigmentation is extensive although absent from the lateral integumentary layers of the trunk and tail. The pattern along the dorsal surface consists basically of two rows of large and medium sized melanophores running from the beginning of the second dorsal to the second and third rays of the third doxsal fin. These are interspersed by very large stellate melarophores which straddle the mid-line and correspond to the original series in the earlier larvae. A series of melanophores is 
present at the base of some dorsal spines (uneven distribution) and all of the dorsal rays. A V-shaped series of small melanophores overlies the first myomeres. The spinal cord melanophores are inter-segmental whereas a series ventral to the vertebral column equates roughly one per centra. Six elongate pigment cells lie on the septum between the hypaxial and epaxial muscles of the caudal peduncle. A diffuse melanic series runs around the posterior margins of the hypurals to link with a very laxge melanophore beneath the caudal peduncle which in turn leads into the ventral series, arranged one per anal ray. Thirteen melanophores run along the belly in line with 3 more which form a triangular pattern about the cleithral symphasis. These are surrounded by several smaller pigment cells. Numerous small integumentary melanophores are scattered above the brain between the eyes and on the snout. The upper and lower jaws have a diffuse array of large and small stellate melanophores which lie in the integument of the fleshy lips. Two melanophores sit beneath each nasal bone and the anterior of the two curves about the dorsal head of the maxillary. The basic series of neural chromatophores is visible close to the brain but an additional large melanophore is present each side of the myelencephalon and is visible through the otic capsules. A broad fan-shaped melanic patch lines the medial wall of the branchial chamber. Two more patches occupy the lateral and dorsolateral surfaces of the chamber. Numerous tiny pigment cells cover the muscular base of the pectoral fin, while another 3-4 border the orbital bones.

\section{Helcogramma sp. 1}

\section{Diagnosis:}

This summary is taken from 13 prejuveniles and juveniles $20-31 \mathrm{~mm}$ S.I.; D..IV, XVI-XVII, 12-13; A. II 22-23; C. 12-13 (unbranched); $P_{I}$. 16-17; vertebrae 42-43; the few lateral line scales which were present plunged quickly to the mid-line beneath the pectoral fin; nasal tentacles 1 ; orbital tentacles none; first dorsal fin continuous with the second.

\section{Larval diagnosis: (20 specimens)}

41-43 myomeres (12-13 pre-anal); sparse pigmentation in late larvae; $15.8 \mathrm{~mm}$ S.L. one melanophore above the mesencephalon and myelencephalon; prejuvenile at about $19 \mathrm{~mm}$ S.L.; meristic counts within range of adults; 13 pre-anal vertebrae. 
Spawning months: Larvae larger than $15.0 \mathrm{~mm}$ s.1. present in plankton from November to January,

The individuals of this larval series (Helcogramma sp.1) have meristic characteristics which fit with the second half of the original description of Helcogramma medium (Gïnther, 1861), where two specimens are described with 23 anal rays, a nasal tentacle, and a first dorsal fin continuous with the second. It is the adult of these larvae which has been commonly accepted as Helcogramma medium (Waite, 1913; Graham, 1953; Darby, 1966). Another larval series following that of Helcogramma sp.2 and designated Helcogramma medium (in part Günther, 1861) meristically similar, but very different in terms of pigmentation, complies closely with the first half of Ginther's (1861) description. The adult of this larval series has not been found.

Larva $15.8 \mathrm{~mm}$ s.1. (Fig.10, no's $1 \&$ la). The elements of the suspensory system are well defined and ossified. The nasal tubes are complete and are distinctly V-shaped in contrast with the U-shaped ones of other tripterygiids. All the fins except the first dorsal and the pelvics have the full adult number of rays and spines. The spines in the second dorsal. fin are low and even while the rays of the third dorsal and caudal fins are segmented (anal rays not yet segmented). There is a medium sized melanophore above the optic lobes, one above the medulla oblongata and 4-5 along the spinal cord beneath the third dorsal fin. Two to 3 elongate pigment cells straddle the bases of the caudal rays and there are two other small ones beneath the caudal peduncle. The series associated with the rays of the anal fin numbers between 19 and 20 cells. The usual melanic patches lie above the gut and at the anterior ventral corner of the liver. On each side of the cleithral symphasis anterior to the pelvic buds is a small stellate melanophore. Larvae this size and larger are present in the surge channel schools.

Prejuvenile $20.2 \mathrm{~mm} \mathrm{s.1.} \mathrm{(Fig.10,} \mathrm{no.2).} \mathrm{The} \mathrm{depth} \mathrm{has} \mathrm{increased}$ considerably producing a more robust overall appearance. Spines of the first dorsal fin are even and the membrane is continuous with that of the second dorsal fin. The anteriormost spines of the second dorsal are the longest descending gradually posteriorly. Spinal cord melanophores have increased in number to form an interdigitated series of about 25 cells. A medium sized melanophore is visible on the isthmus of the throat and a medial group of small pigment cells lies scattered above the maxillae and 
premaxillae. An additional medium sized melanophore is present on the right side of the mesencephalon and along the dorsal mid-line associated with the rays are 11 melanophores. Large melanophores straddle the second dorsal fin, two halfway along and 4 around the last spine. Prejuveniles about 19-20 mm s.1. pigment rapidly and begin to settle out of the plankton. The pattern just described may be rapidly added to, first about the head then along the dorsal mid-line and finally over the lateral integument of the trunk and tail.

Prejuvenile $21.8 \mathrm{~mm} \mathrm{5.1.} \mathrm{(Fig.10,} \mathrm{no.3).} \mathrm{The} \mathrm{head} \mathrm{is} \mathrm{low,} \mathrm{the}$ snout is rounded and the eyes do not as yet interrupt the dorsal profile. The maxillaries pass obliquely back to a point just behind the anterior margin of the eye. Each anterior nostril has a simple tentacle on the posterolateral margin and the posterior nostrils lie close to the eyes. The first dorsal fin is half as high as the second which is two thirds the height of the third. The second and third rays of the first dorsal fin are the longest and the nembrane remains continuous with that of the second dorsal fin. Posteriorly the caudal fin is notched, the abdomen is deep and rotund and the base of the pectoral fin is broad. The lowest rays of the latter are thickened and the few scales present on the anterior part of the lateral line plunge rapidly to the mid-line. A large melanophore is placed centrally above the mesencephalon, associated with about 11 medium and numerous small sized ones which outline the major lobes of the brain. Many tiny pigment cells are present in the integument about the anterior nostrils, above the mesethmoid and medially on the upper and lower lips. Beneath the eyes the small melanophores are arranged in bars which radiate ventrally from the lower margin of the eye across the elements of the jaws and cheek muscles. Two elongate, parallel clusters pass anteroventrally from the first myomere across the otic capsule. A group of melanophores overlies the operculum, appearing continuous with another series beneath the cheek muscles on the preoperculum. A characteristic (of prejuvenile tripterygiids) V-shaped group is present on the base of the pectoral fin, adjacent to another cluster at the base of the pectoral rays. The typical series of pigment cells runs along the proximal ends of the third dorsal and anal rays interspersed by many tiny cells. A large stellate melanophore on the ventrolateral surface of the myelencephalon radiates to meet the pigment cell above the medulla. There are 6 well defined melanic patches along the dorsal mid-line which consists of cells of varying sizes. The 
larger of these pigment cells are linearly arranged each side of the second dorsal fin. All six patches extend laterally to meet 7-8 similar ones on the dorsolateral aspect. Extending over the mid-lateral integument from the base of the pectoral fin to the hypurals are 9-10 square patches of small and medium sized melanophores. The last group covers most of the caudal peduncle. Nine more patches run along the ventrolateral aspect slightly offset from the row above. These may be square, H-shaped or merely vertical stripes (particularly beneath the pectoral fin). The spinal melanophores form a complete series except for those above the last 3 centra and several pigment cells have appeared on the arteries and veins beneath the vertebral column. Numerous tiny melanophores border the anal and caudal rays.

Juvenile $21 \mathrm{~mm} \mathrm{~s} .1$. (Fig.11, no's $2 \& 2 a$ ). The juvenile condition is recognized by the degree of pigmentation and sedentary habit rather than size. Many juveniles are slightly smaller than some unpigmented, freeswimming prejuveniles. Soon after settling out of the plankton the forehead becomes steepex and the eyes interrupt the profile. The upper jaw overlaps the lower and they pass back horizontally to terminate level with the anterior edge of the pupil. The overall proportions approach those of the adult except that the caudal fin has a straight posterior margin compared with the round condition of more mature individuals. The basic pigmentation pattern is unchanged although the total number of pigment cells has increased. All the barred patterns radiating from the eye extend onto the iris. Groups of guanophores lie between the melanic patches (figured as tiny dots Fig.11, no's 2 \& 2a) producing a chequerboard effect particularly along the lateral. integument. The mid-lateral patches of melanophores join in older individuals forming a zig-zag line along the body. A line of isolated pigment cells xuns each side of the anal rays and numerous tiny melanophores form oblique bars in the second and third dorsal fins. The dorsal edge of the first dorsal has a dark fringe. 


\section{Helcogramma sp. 2}

\section{Diagnosis:}

This summaxy is taken from 18 prejuveniles $14-20 \mathrm{~mm}$ S.L.; D. IV, XIV-XVI, 9-11; A. 20-22; C, 12-13 (unbranched); $\mathrm{P}_{I} \cdot 15-17$; vertebrae 40-4l; nasal tentacles 1 ; orbital tentacles none; only 4-5 lateral line scales present showing a definite plunge toward the mid-line; spines of the first dorsal fin descendant; no spine preceding the anal rays.

\section{Larval diagnosis: (27 specimens)}

40-41 myomeres (11-12 pre-anal); 7-8 xanthophores around posterior end of notochord; melanophores in row along belly; melanophores ventrolateral to myelencephalon; 7 melanophores beneath caudal peduncle; prejuvenile at about $15 \mathrm{~mm}$ S.L.; meristic counts within range of adults; 12 pre-anal vertebrae.

Spawning months: Larvae $10.0 \mathrm{~mm} \mathrm{s.1}$. and larger present in plankton during November and December.

Larva $11.2 \mathrm{~mm} \mathrm{s.1.} \mathrm{(Fig.12,} \mathrm{no's} \mathrm{1,} \mathrm{la} \mathrm{\&} \mathrm{1b).} \mathrm{The} \mathrm{brain} \mathrm{extends}$ forward of the anterior margin of the eyes and the olfactory cups are not curled at the edges. The third dorsal, caudal and anal fins have 11-12, 13 and 20 weakly ossifjed rays respectively. Those of the caudal fin axe faintly segmented. A sinall melanophore lies above the junction of the telencephalon and the mesencephalon, a large one above the mesencephalon and myelencephalon and a series of 16-17 medium sized ones run along the spinal cord. Several erythrophores and xanthophores surround the brain, imparting a red-yellow hue to nervous and adjacent tissues. Directly beneath the third dorsal fin close to the vertebral column are 7-8 stellate xanthophores, which produce a characteristic yellow patch. Each xanthophore sits above a space between the centra and remain there throughout the larval development. There are 20 melanophores along the anal fin continuous with a typical row of 5-7 beneath the caudal peduncle. Elongate interdigitating pigment cells border the hypurals and 5-7 small ones xun along the dorsal mid-line beneath the rays of the third dorsal fin. The abdominal and cleithral pigmentation is diagnostic. There is a row of 2-4 melanophores along the belly associated with 3-4 scattered erythrophores (small dots Fig.12, no.1b). A stellate melanophore is visible either side of the cleithral symphasis in front of two erythrophores placed each side of the heart. Iwo small melanophores axe present in the integument above the junction of the maxillae, and the usual melanic patch surrounds the anterior ventral corner of the liver. At 10.7m s.1. the laxva is identical except for a lower number of melanophores along the spinal cord (5-6), 
grouped below the third dorsal fin.

Larva $14.8 \mathrm{~mm}$ s.1. (Fig.12, no's 2, 2a \& 2b). The general shape at an equivalent size is similar to Helcogramma species 1 and the two are readily confused if pignentation has faded. Differences in vertebrae, anal ray counts and the presence or absence of anal spines distinguish the two species. The brain no longer extends forward of the eyes and each nasal tube is distinctly $V$-shaped. The spines of the second dorsal fin are low and slightly smallex posteriorly. Neural pigmentation (Fig.12, no.2a) is unchanged except for the expansion of the pigment cell above the telencephalon and the appearance of a medium sized melanophore on the ventrolateral surface of the myelencephalon. Characteristically 7 melanophores run beneath the caudal peduncle, two border the hypurals and 4 lie at the base of the spines half way along the second dorsal fin. More melanophores and erythrophores are present on the belly and the central melanic row has divided near the anus (Fig.12, no.2b). The number of pigment cells on the isthmus of the throat has increased and their relative positions are best seen on Fig.12, no.2b. Hypaxial abdominal muscle flaps are not fully joined along the ventral mid-line.

Prejuvenile $16.9 \mathrm{~mm}$ s.1. (Fig.12, no's 3 \& 3a). The spines of the first dorsal fin are descendant and the membrane is not continuous with that of the second. The body is elongate and narrow, contoured precisely along the dorsal and ventral profiles and the caudal peduncle is characteristically long and thin. There is a spatulate tentacle on the posterior margin of the anterior nostril. The bones of the neurocranium and the suspensorium are well defined and ossified. An additional pair of small melanophores is present medially on the premaxillary lip. The spinal melanophores have increased to 18 and those beneath the dorsal fins to about 11. The latter form an interrupted series always at the base of the dorsal rays and spines and on the caudal peduncle. In the previously described Helcogramma species the melanophores develop anteriorly as rows either side of the second dorsal fin. The corona of yellow persists in the tissues about the brain, posterior vertebral column and is also present along the jaws. One to two small melanophores lie on the lateral surface of the branchial chamber and two more elongate ones border the hypurals. 
Prejuvenile $18.0 \mathrm{~mm}$ s.1. (Fig.11, no's 1, la \& 1b). The depth has increased producing a robust appearance to the head and abdomen. The first dorsal fin composed of descendant spines is half the height of the second which is two thirds that of the third. All the rays are faintly segmented. There are no spines or vestiges of pterygiophores preceding the anal rays and the ventral rays of the pectoral fin are thickened. Bony elements of the neurocxanium, preoperculum and orbital series show well developed canals which carry the lateral line system. The anterior nostril is tubular and the tentacle is larger. The melanophores along the dorsal mid-line have increased to about 19 arranged in 4 groups, two of 5-6 cells beneath the second dorsal fin, one of 3-4 cells between the second and third dorsal fins and another group beneath the posterior rays of the third dorsal fin. One melanophore sits on the dorsal mid-line of the caudal peduncle and those on the belly have increased to about 17 (Fig.11, no.1b).

\section{Helcogramma medium (in part Günther, 1861)}

\section{Diagnosis:}

This sumnary is taken from 21 prejuveniles and juveniles 13.5$18.0 \mathrm{~mm} \mathrm{S.L.;} \mathrm{D.} \mathrm{IV,} \mathrm{XV-XVI,} \mathrm{11-12;} \mathrm{A.} \mathrm{II,} \mathrm{20-21;} \mathrm{C.} \mathrm{12-13} \mathrm{(unbranched);}$ $\mathrm{P}_{\mathrm{T}}$. 15-16; vertebrae 39-40; nasal tentacles 1; orbital tentacles none; approximately 19 lateral line scales present, and these plunge quickly to the mid-line beneath the pectoral fin.

\section{Larval diagnosis: (27 specimens)}

39-40 myomeres (11-12 pre-anal); 7-8 xanthophores around posterior end of notochord; melanophores in row along belly; melanophores ventrolateral to myelencephalon; 5 melanophores beneath caudal peduncle; prejuvenile at about $13 \mathrm{~mm}$ S.L.; meristic counts within range of adults; 11-12 pre-anal vertebrae.

Spawning months: Larvae $11.0 \mathrm{~mm}$ S.L. and laxger present from November to January.

This series is very similar to that of Helcogramma species 2. The larvae of $\mathrm{H}$. medium develop features such as rays and spines at a smaller size than either of the two previously described Helcogramma species. This suggests that the adult (not found) of H. medium may be smaller than those of the other two (see discussion - larval skeleton). 
Larva $11.2 \mathrm{~mm} \mathrm{s.1.} \mathrm{(Fig.13,} \mathrm{no's} \mathrm{1,} \mathrm{la} \mathrm{\&} \mathrm{1b).} \mathrm{The} \mathrm{rays} \mathrm{of} \mathrm{the}$ third dorsal, caudal and anal fins are well defined and ossified at the bases. The edges of the olfactory cups have curled inward and almost meet to form the nasal tubes. Two melanic patches lie directly above the strongly coiled gut which laterally is a faint red-orange colour. Numerous xanthophores surround the brain and 7-8 are present beneath the spinal cord under the third dorsal fin. A large and a medium sized melanophore lie above the mesencephalon, and another large one on the myelencephalon sends extensions around the cerebellum and a row of 24 lie along the spinal cord. The 3 anterior spinal melanophores form a separate group close to the melanophore above the medulla oblongata. On the ventrolateral surface of the rnyelencephalon are two large melanophores, visible through the otic capsules. The large pigment cell above the mesencephalon is typically on one side of the mid-line compared with the other two species of Helcograma. A group of small melanophores is present on the medial wall of the branchial chamber halfway along the cleithrum. Ten melanophores form a continuous series at the base of the rays in the third dorsal fin. The usual pigment cells border the hypurals and there are 5 melanophores along the ventral mid-line of the peduncle. Twenty melanophores form an uninterrupted series at the base of the anal rays and two are present on the cleithral symphasis closely associated with 4 erythrophores. Several melanophores and exythrophores lie scattered over the belly, and in some individuals above the maxillary and premaxillary.

Prejuvenile $16.5 \mathrm{~mm} \mathrm{s.1.} \mathrm{(Fig.} \mathrm{13,} \mathrm{no's} \mathrm{2,} \mathrm{2a} \mathrm{\&} \mathrm{2b).} \mathrm{Individuals}$ $11.0 \mathrm{~mm}$ and larger are abundant in the surge channels. The spines of the first dorsal fin are low and even and the membrane is not continuous with that of the second. The first dorsal fin is half the height of the second which is half the height of the third. Elements of the anal and second dorsal fin decrease gradually posteriorly. Pigmentation is laid down rapidly at about this size. From 3-12 melanophores and 0-7 exythrophores are arranged haphazardly along the mid-line of the belly dividing posteriorly just before the anus and another small melanophore has been added to those already present at the cleithral symphasis. Some individuals have two medium sized pigment cells transversely placed halfway along the belly. Two large pigment cells cover the cleithral surface of the branchial chamber. The bxain is contracted, broader and typically peax-shaped. Above the midline between the optic lobes there is a large melanophore which is not 
216.

displaced to one side as in earlier stages (Fig.13, no.2a). Associated with this are 4-8 medium sized melanophores on the dorsolateral surfaces of the optic lobes. The telencephalon is obscured by as many as 10 small melanophores. Numerous xanthophores (figured as grey chromatophores) surround the entire brain producing a distinct corona of yellow. There is no pigment cell above the telencephalon as in the other two Helcogramma species. Several small melanophores are aggregated above the medial. ends of the premaxillaries and maxillaries. Three melanic groups are present on the dorsal mid-line, two groups of 4 cells halfway along the second dorsal fin and another of 6 cells between the second and third dorsal fin. Each melanophore associated with the rays of the third dorsal fin has 3 extensions, two which project anterolaterally about a preceding ray and one which extends posteriorly along the mid-line. Those with the anal rays are similar except the extensions are shorter and additional portions pass into the muscle tissue above. Chromatophores above and below the caudal peduncle are bilaterally symetrical with an elongate axis running along the mid-Iine. Spinal melanophores form a continuous interdigitated series which cannot be accurately counted.

In typical tripterygid fashion further chromatophoxes are added to the head, then along the dorsal surface and ultimately to the lateral integument. Individuals between 17 and $18 \mathrm{~mm} \mathrm{s.1.,} \mathrm{in} \mathrm{addition} \mathrm{to}$ existing chromatophores, have distinct clusters of erythrophores and xanthophores uniformly distributed above the mesencephalon and the telencephalon. Each cluster contains approximately 13 tiny chromatophores. Numerous tiny melanophores lie in the integument above the olfactory tubes, the premaxillary and the maxillary lips. There are now 10 patches along the dorsal mid-line extending onto the caudal peduncle. The tiny melanophores lateral to the head appear concentrated on the otic capsules, the opercular flap, internal walls of the branchial chamber and on the muscular base of the pectoral fin. Tiny melanophores are also lightly scattered over the lateral surfaces of the trunk and tail. The pigmentation on the belly is unchanged although the posterior division of the linear series is more pronounced.

Prejuvenile $18.0 \mathrm{~mm}$ s.1. (Fig.13, no's $3 \& 3 a$ ). There is a pronounced similarity between these prejuveniles and those of Helcogramma species 1, in terms of the head and body profile and the overall 1 pigmentation. The first dorsal fin is however proportionately higher 
in $\mathrm{H}$. medium, nearly two thirds the height of the second, the lateral and dorsal pigmentation is present at a smaller size (17-18 mm cf. 20-21 mm s.1.) and the posterior nostril is distinctly tubular. The two species are separated further by differences in meristic counts. Along the dorsal. mid-line are 5-6 patches of medium and small sized melanophores. The anterior one forms a bar across the nape, the second and third 0-shaped patches, the fourth and fifth transverse H-shapes and the sixth is a simple bar straddling the caudal peduncle. The melanophores above the brain have increased in number outlining the lobes. No xanthophores or erythrophores were visible on the preserved specimens except as faint grey cells. The medial large melanophore is present in most individuals directly above the optic lobes. There are few tiny melanophores in the integument between the eyes but some are present around the nostrils and on the upper lip. Those overlying the otic capsule appear as parallel bars which extend onto the opercular flap. A V-shaped patch covers the base of the pectoral fin extending onto the ventral rays. The cheek is free of pigmentation although any melanophores originally present may have faded in formalin. Two rows of square and partly triangular melanic patches run along the lateral integument from beneath the pectoral fin to the caudal peduncle. The patches in each row are offset slightly and joined to form oblique bars which blend dorsally with a hazy dorsolateral series of melanophores. These in turn link with the patches along the dorsal surface. The ventral portions of the oblique bars also join along the mid-line to form a zig-zag pattern in the best preserved specimens. Many tiny melanophores form oblique stripes in the second dorsal fin and anteriorly on the caudal rays. Belly, cleithral, anal and spinal pigmentation is unchanged from earlier stages.

\section{- Tripterygion segmentatum McCulloch \& Phillipps, 1923}

\section{Adult diagnosis: (8 specimens)}

Maximum S.I. 31mm; D. IV, XVII-XVIII, 12-13; A. I 24; C. 11-12; $P_{1}$. 17; vertebrae 42; nasal tentacles 1 ; orbital tentacles 1 ; lateral line 23-24 scales which follow the dorsal profile; adult vividly pigmented with red and blue. during November.

Spawning months: Prejuveniles present in harbour plankton 
Tripterygion segmentatum is meristically similar to the undescribed "Yaldwyn's blenny", but is distinguished by the presence of large nasal and orbital tentacles and descendant spines in the first dorsal fin. No stages earlier than prejuvanile were collected.

Prejuvenile (description is taken from 4 individuals 16.5, 17.2, 17.3 and $17.5 \mathrm{~mm} \mathrm{s.1.).} \mathrm{The} \mathrm{overall} \mathrm{proportions} \mathrm{are} \mathrm{the} \mathrm{same} \mathrm{as} \mathrm{those} \mathrm{of}$ the adult (Graham, 1953) and both tentacles are present. The only scales formed are the first $4-5$ on the lateral line. Considering the degree of ossification and the length relative to that of the adult (31 mm s.1.) the amount of pigmentation is sparse. The only melanophores on the trunk and tail are those associated with the anal rays, except the last divided one. Above the brain there is a pronounced red tinge which spreads laterally and ventrally behind the eyes. Individual erythrophores responsible for this colour are indiscernable. This colour is very intense above the mesencephalon and telencephalon, and pervades the surrounding tissues. Three of the 4 specimens have two small melanophores well back on the mesencephalon, one on each lobe. Another is present above the pineal body. Several tiny melanophores are clustered on the medial portions of the premaxillaries while many others form two bars radiating from the ventral curve of the eye. One passes across the lachrymal onto the naxillary and the other over the cheek muscles. A wedge shaped group of small piginent cells is present within the branchial chamber directly under the hyomandibula and operculum. Several widely distributed melanophores cover the muscular base of the pectoral fin. Along the abdomen and throat are precisely arranged erythrophores. A line of these tiny cells begins on the isthmus passing posteriorly and dividiny on the cleithral symphasis to run onto the base of the pectoral fin. Two other widely separated rows run along the abdomen to the anus. The hypurals are bordered posteriorly by several small exythrophores.

\section{New tripterygiid species}

\section{Diagnosis:}

Summary compiled from 4 larvae and one prejuvenile; D. III, XIV-XV, 10-11; A. 19-20; C. 12-13; $\mathrm{P}_{I}$. 14; vertebrae 36-38; 10-11 pre-anal vertebrae; $8.0 \mathrm{mn}$ S.L. notochord upturned; broad band of erythrophores in integument of trunk. 
The following larval series is compiled from only 6 individuals collected off Island Bay on 6.12.71. The pigmentation is unusual and different fxom other known tripterygiid larvae. The larvae do not belong to any formally described or commonly named species although the larval form and meristic counts are close to those of Gilloblennius tripennis. It is also meristically similar to an undescribed species found in wellington Harbour (Mr J. Moreland pers. comm.) which cannot be placed in Gilloblennius because it has an interxupted lateral line and therefore requires a new genus. I have no information about the lateral line of these larvae and therefore hesitate to refex them to any genus.

Larva $8.0 \mathrm{~mm}$ s.1. (one specimen, Fig.14, no.l\& \& la). The head is large and bulbous and the gut is strongly coiled. The telencephalon extends forward of the eyes and the olfactory cups have not yet curled at the edges. The width of the body at the first myomeres is considerably less than that at the otic capsules. Dorsal and ventral fin folds are continuous with the caudal lobe and the urostyle is upturned slightly. The hypurals carry 5 weakly ossified rays. Each pectoral fin is a transparent spatulate flap and the jaws are ossified. A large melanophore is present on the inner surface of the chondrocranium above the mesencephalon, another medium sized one above the telencephalon and an elongate melanophore lies above the myelencephalon sending extensions beneath the cerebellum. Seven elongate melanophores are unevenly distributed along the spinal cord. There are two small pigment cells above the caudal peduncle and below the hypurals. Sixteen small melanophores run along the ventral mid-line as an irregular series from the anus to the caudal peduncle. The upper parietal peritoneum has two confluent melanic patches which overlie a small airbladder. Another patch is visible surxounding the anterior ventral corner of the liver. Two melanophores are present on the belly and another on the isthmus of the throat. The most diagnostic feature of the whole series is the red tinge which pervades the muscle tissue above the gut. This extends from behind the brain to the anus and is produced by numerous tiny erythrophores (figured as tiny black pigment cells). Many xanthophores accompany the erythrophores above the gut and around the brain imparting a yellow tinge to these areas. There are 37-38 myomeres 1.1 of which are pre-anal. 
Larva $11.5 \mathrm{~mm} \mathrm{s.1.} \mathrm{(two} \mathrm{specimens,} \mathrm{Fig.14,} \mathrm{no's} \mathrm{2,} \mathrm{2a} \mathrm{\&} \mathrm{2b).} \mathrm{The}$ well defined jaws are fleshy and each nasal tube is complete but without dermal tentacles. The head is broad and tapers gradually onto the muscles of the trunk and tail. This broadening is accompanied by a shortening of the brain which no longer extends forward of the eyes. The partially ossified chondrocranium is clearly visible about the brain and the suspensorium is well ossified. Twelve to 13 segmented rays are supported by the hypurals beneath the fully upturned urostyle. The second and third dorsal fins have 15 spines and 10-11 rays respectively, each ossified at the base. Nineteen to 20 anal and 14-15 pectoral rays are visible. Chromatophores above the brain are unchanged whereas those along the spinal chord have increased to 17-18 with the anterior ones interdigitated (Fig.14, no.2b). The melanophores associated with the hypurals are evenly spread along the posterior vertical margins while those along the anal fin are irregular with 9 in one specimen and 20 in the other. One medium sized melanophore sits above and one below the caudal peduncle on the midlines. Erythrophores have increased on the trunk forming a broad band from the belly to the dorsal mid-line. These also extend along the proximal ends of the pectoral rays. A characteristic series of 11-12 medium sized, stellate melanophores runs in two rows in the integument of the belly. Seven to 8 more form a $\mathrm{V}$-shaped pattern at the cleithral symphasis. The hypaxial abdominal muscle flaps are not yet closed (F'ig.14, no.2a).

Prejuvenile $14.6 \mathrm{~mm}$ s.1. (one specimen, Fig.14, no's 3 \& 3a). The anterior portion of the body is rotund. The upper jaw overlaps the lower and passes obliquely back to terminate beneath the anterior margin of the pupil. A small tentacle is present on the posterior margin of the anterior nostril and the whole nasal tube is tucked in close to the eyes. A larger simple tentacle sits above the centre of the eye. The 3 spines of the first dorsal fin are descendant while those of the second decrease gradually posteriorly and the longest are a third the height of the third dorsal fin. Twelve segmented and 6-7 dorsal and ventral procurrent rays are visible in the caudal fin which is notched on its posterior margin. No spine is visible in front of the 20 anal rays even though all of these are ossified proximally. The pelvic rays are thick at the base tapering posteriorly and finishing level. with the anterior margin of the anus. Each pectoral fin is broad and spatulate with the lower rays typically thickened. The melanophores above the brain are very large covering most 
of the telencephalon and mesencephalon. An extension from the one above the mesencephalon passes posteriorly onto the metencephalon (cerebellum). All the pigment cells above the spinal cord and myelencephalon have joined making accurate counting impossible. Six medium sized melanophores are present below the first rays of the third dorsal fin and 3 more sit along the dorsal and ventral mid-lines of the caudal peduncle. A full series of 20 melanophores accompanies the anal rays. The erythrophores are more numerous about the abdomen and on the pectoral fin having spread posteriorly beyond the anus and anteriorly onto the nape, lower cheek and upper jaw: the overall colour is almost crimson. Two medium sized melanophores are visible on the ventrolateral surface of the myelencephalon. The melanophores on the belly are arranged in the same pattern as in smaller individuals but have increased to 16 and become more stellate and larger. There are 8 similar ones at the cleithral symphasis. The parietal peritoneum of the abdorinal cavity is extensively covered by a melanic patch which in earlier specimens was divided into 5 separate patches. Two lay above the gut, another surrounded the anterior ventral corner of the livex and one occupied each anterolateral corner of the abdominal cavity. Gradual expansion brought the 5 together as one patch.

Family Clinidae

Subfamily Labrisominae

Tribe Notoclinini.

Notoclinus compressus (Hutton, 1872)

Adult diagnosis: (10 specimens)

Maximum S.L. $65 \mathrm{~mm} ;$ D. IV, X-XI, 9-11; A. I 18-20; C. 9; P-10-11; vertebrae 35-36; nasal tentacles 1 ; orbital tentacle 1 spatulate and large; lateral line scales 17-18; three dorsal fins with spines of the first dorsal descendant.

Larval diagnosis: (25 specimens)

35-37 myomeres ( $9-10$ pre-anal); gut strongly coiled; $7.5 \mathrm{~mm}$ S.I. notochord upturned; melanophores present on anterolateral wall of abdominal cavity; no spinal melanophores; no melanophore above telencephaion; prejuvenile at about $11.0 \mathrm{~mm}$ S.L.; meristic counts within range of adults; pre-anaj vertebrae 10-11.

Spawning roonths: late August to December. 
The larval stages of $\mathrm{N}$. compressum and $\mathrm{N}$. fenestratus are similar to those of the tripterygiids, but differ in having a very low number of pre-anal myomeres (9-10). They also have a characteristic pair of widely spaced melanic patches on the peritoneal septum between the pericardial and abdominal cavities (Fig.16, no.3b). They share this feature with the new tripterygiid species just described.

Larva $6.4 \mathrm{~mm} \mathrm{s.1.} \mathrm{(Fig.15,} \mathrm{no's} \mathrm{1,} \mathrm{la} \mathrm{\&} \mathrm{1b).} \mathrm{The} \mathrm{head} \mathrm{is} \mathrm{bulbous}$ and blunt exaggerated by the strongly coiled gut. Each olfactory cup is shallow and the cleithrum is visible as a refractive line running obliquely from the second myomere to the anterior ventral corner of the liver. The brain is narrow, well defined and extends forward of the anterior margin of the eyes. The upper jaw overlaps the lower and the caudal. anlage is present beneath the straight notochord of the tail. Dorsal and ventral fin folds are continuous with the spatulate caudal lobe, although there is a definite constriction near the future caudal peduncle. Two melanic patches lie above the gut and two on the visceropericaxdial septum and with the one at the anterior ventral corner of the liver they form a triangulax pattern (Fig.15, no.1b). A tiny melanophore is present on the caudal anlage and 14-15 small ones run along the ventral mid-line of the tail: the anterior of these are typically higher in the muscle tissue. There is a medium sized melanophore above the mesencephalon.

Larva $8.5 \mathrm{~mm} \mathrm{s.1.} \mathrm{(Fig.15,} \mathrm{no's} 2$ \& 2a). These larvae are superficially similar to equivalent stages of $\mathrm{G}$. tripennis except the gut is more bunched to span only 9-10 myomeres (cf. 10-12) and there is a pair of visceroperitoneal melanic patches. The olfactory cups are curled at the edges. The notochord is upturned in the tail continuing into the caudal. lobe. Two elongate melanophores border the hypurals which carry 8-10 weakly ossified rays. Ten third dorsal and 18 anal rays are visible as strips of tissue at the base of the dorsal and ventral fin folds. The sequence of ossifjcation is the same as that in Forsterygion varium. Each pectoral flap is broad and is situated high on the body. In addition to the typical melanic patches in the parietal peritoneum another one is present halfway up the cleithrum and obscured by the former. Three tiny melanophores form a triangle on the cleithral symphasis forward of the pelvic buds. A row of 10-18 spindly melanophores lie deep in the muscle above the anal fin fold. The two above the caudal peduncle are elongate and integumentary. The lateral peritoneum of the gut cavity has an iridescent blue sheen and 
the hindbrain and otic capsules are surrounded by a yellow corona. There are no spinal melanophores.

Prejuvenile $11.7 \mathrm{~mm} \mathrm{s.1.} \mathrm{(Fig.} \mathrm{15,} \mathrm{no's} 3 \& 3 a$ ). The spines of the first dorsal are low but distinctly descendant. The profile beneath the second dorsal fin is smooth and gently sloped while that below the third and above the anal fin is serrated. The brain is broad, contracted longitudinally and extends just forward of the anterior margin of the pupil. Anteriorly the head and trunk are distinctly rotund, the lips are fleshy and the nasal tubes U-shaped. Orbital tentacles are visible as small swellings in the integument above each eye. The base of the pectoral fin is fleshy and the web is broad extending well past the anus. The pelvic rays are long and slender with the longest reaching a point halfway along the abdomen. Pigmentation is unchanged except for the enlargement of the melanic patches in the parietal peritoneum which now overlap considerably. Nine small melanophores lie scattered over the belly and the pigment cell above the mesencephalon covers most of the lobes. A blue sheen surrounds the hindgut but there is also a dull red hue to the tissues near the major coil of the gut. Three small. melanophores are associated with the middle rays of the third dorsal fin. The yellow tinge in the tissue about the brain is more intense and has spread to the muscles above the gut.

Prejuvenile $12.8 \mathrm{~mm}$ s.1. (Fig.15, no.4 and Fig.16, no.1). The bony elements of the skull are well defined and the posterior ends of the maxillary and premaxillary slide beneath the fleshy circumorbitals. The head is broadest at the operculum and the trunk and tail have increased in depth giving a more robust appearance. Each orbital. tentacle is spatulate. The first spine of the first dorsal fin is as long as those of the second which are half the height of the rays in the third dorsal fin. The longest pelvic ray teminates level with the anterior margin of the anus and the upper edge of the branchiostegal flap is serrated. Individuals about this size and larger are present in the surge channels. The melanic patches in the parietal peritoneum obscure all but the lowest portions of the gut. Melanophores on the belly range in number from 4-9 while those along the anal fin from 11-20. Between 4 and 10 small melanophores are irregularly arranged along the dorsal mid-line. Two to 3 spindly pigment cells lie embedded high in the muscle above the anal 
fin while several tiny erythrophores are scattered over the lower operculum and close to the 3 melanophores on the cleithral symphasis. Small melanophores are also scattered over the integument of the cheek and muscular base to the pectoral fin. The large pigment cell above the mesencephalon sends two dendritic extensions onto the telencephalon as well as the one passing posteriorly over the cerebellum. The large elongate melanophore above the myelencephalon sends anterolateral projections across the posteromedial surfaces of the otic capsules. Three poorly defined melanophores lie on the ventrolateral surface of the myelencephalon and there are typically no pigment cells along the spinal cord. The yellow around the brain and the red and blue about the gut still persist while the melanic patch on the cleithrum is more elongate.

Prejuvenile $13.5 \mathrm{~mm}$ s.1. (Fig.16, no's 2, 2a, 2b \& 2c). The overall shape has changed little except for an increase in the depth of the abdomen. Each anterior nostril is tubular and beaxs on its posterior margin a small tentacle. An elongate spatulate tentacle is present above each eye and the fourth spine of the first dorsal fin is set apart from the anterior 3 (Fig. 16, no.2b). The fins are unchanged except for a slight thickening of the lower pectoral rays. Pigmentation proceeds rapidiy at this stage over the head, along the dorsal. and ventral mid-lines and then onto the lateral integument. Ten to 11 melanophores run along the third dorsal fin and 16-18 along the anal fin. The pigment cell along the cleithrum spans the full depth of the muscular base of the pectoral fin. A broad patch of many small melanophores is present on the inner surface of the hyomandibula extending back onto the medial surface of the preoperculum and operculum. Three bars composed of small and medium sized melanophores radiate from the ventral curvature of the eye; one across the lachrymal onto the premaxillary, one from the middle of the eye to the articulation point of the jaw and another crossing the cheek onto the preoperculum. All of these continue onto the iris of the eye. Numerous melanophores cover the base of the pectoral fin passing around the muscle onto the axillary surface. A patch of tiny melanophores arch around the proximal ends of the pectoral rays and 5 small stellate melanophores form a T-shaped pattern on the belly. A transverse bax of tiny melanophores cross the proximal ends and another crosses halfway along the pelvic rays. The usual triangular pattern of 3 pigment cells covers the cleithral symphasis although some individuals have only a medial one. 
Juvenile $14.4 \mathrm{~mm}$ s.I. (Fig.16, no.4). A single specimen was collected from the fronds of a Bryopsis species of brown algae. The overall shape and fin structure is sirnilar to that of the adult although the body is slightly less robust. The nasal and orbital tentacles are well developed and pigmentation is not present on the lateral integument of the trunk and tail. The patches of pigmentation are composed of hundreds of small melanophores (brownish) closely associated with an equal number of xanthophores. Patches on the head are concentrated above the lobes of the brain but continue laterally onto the iris of the eye. There are two sets of melanophores on the head, those on the inner surface of the neurocranium, a carxy over from earlier stages, and those in the integument. The anterior 2 bars radiating from the ventral curvature of the eye have intensified. Irregular patches cover the otic capsules, the operculum and the muscular base of the pectoral fin. Each dorsal $f$ in is covered by irregular patches which link with a triangular series along the dorsal mid-line. A similar triangular sequence runs along the ventral mid-line and 6 bars arch dorsoventraily across the rays of the pectoral fin. The tiny pigment cells in these bars are clustered about each ray. An uneven array of bars and patches is present on the caudal fin. The dorsal surface of the spinal cord is covered by many patches which are directed ventrally as triangular wedges. The peritoneum of the gut cavity is heavilypigmented (not figured) and a series of small melanic patches run along each pelvic ray.

\section{Notoclinus fenestratus (Forster, in Bloch and Schneider,} 1801)

\section{Adult diagnosis: (4 specimens)}

Maximum S.I. $140 \mathrm{~mm}$; D.IV, X-XI, 12-13; A. I 21-23; C.9; P ; 11-12; vertebrae $38-40$; nasal tentacles 1 elongate; orbital tentacles 1 I large spatulate; lateral line scales 16-19.

Larval diagnosis: (25 specimens)

38-40 myomeres (9-10 pre-anal); gut strongly coiled; $8.5 \mathrm{~mm}$ S.L. notochord upturned; melanophores present on anterolateral wall of abdominal cavity; spinal melanophores present at $8.5 \mathrm{~mm}$ S.L.; one melanophore above telencephalon at $8.5 \mathrm{~mm}$ S.L.; prejuvenile at about $13.0 \mathrm{~mm}$ S.L.; meristic counts within range of adults; 10-11 pre-anal vertebrae. 
The larval development of $\mathrm{N}$. fenestratus is very similar to that of $\mathrm{N}$. Compressus. Notoclinus fenestratus is larger than $\mathrm{N}$. Compressus which means that its larvae are larger than those of $\mathrm{N}$. compressus at any given level of development e.g. of rays and spines etc. (see discussion larval skeleton). Notoclinus fenestratus is distinguished further by having a melanophore above the telencephalon and a series along the spinal cord early in development. Larvae and prejuveniles are abundant in the plankton during July, August and September earlier than those of $\mathrm{N}$. compressus which appear between late August and December.

Larva $5.3 \mathrm{~mm} \mathrm{s.1.} \mathrm{The} \mathrm{head} \mathrm{is} \mathrm{blunt} \mathrm{and} \mathrm{bulbous} \mathrm{and} \mathrm{the} \mathrm{gut} \mathrm{is}$ strongly coiled, almost spherical. There are 39-40 myomeres of which 9-10 are pre-anal. The tight1y coiled gut is typical of larvae of $\mathrm{N}$. fenestratus and $\mathrm{N}$. compressus distinguishing them from superficially similar tripterygiid larvae where the hindgut extends some distance beyond the major coil. The notochord is straight. Two melanic patches are visible above the gut, two on the viscero-pericardial septum and one surrounds the anterior ventral corner of the liver. A row of 17-18 tiny melanophores runs along the ventral mid-line of the tail beginning at the tenth myomere past the anus and continuing well down into the tail. The anterior cells in this series are typically embedded high in the muscle. A tiny melanophore is present beneath the notochord in the caudal lobe and therc is a large melanophore above the mesencephalon which in many individuals extends back across the cerebellum and myelencephalon. Larvae of $\mathrm{N}$. compressus at a similar level of development lie between 4 and $5 \mathrm{~mm} \mathrm{s.1.}$

Larva $6.2 \mathrm{~mm} \mathrm{s.1.} \mathrm{The} \mathrm{notochord} \mathrm{is} \mathrm{straight} \mathrm{although} \mathrm{the} \mathrm{anlage}$ of the caudal rays is present.

Larva $8.5 \mathrm{~mm}$ s.1. There are 7 faintly ossified caudal rays beneath the upturned notochord. A melanophore is present above the myelencephalon and the telencephalon and a series of 5-11 pigment cells runs along the spinal cord. At about $8.5 \mathrm{~mm} s .1$. the larvae of $\mathrm{N}$. compressus have vertical hypurals bearing 10 rays and nearly all the dorsal and anal rays are faintly visible.

Larva $9.8 \mathrm{~mm} \mathrm{s.1.} \mathrm{(Fig.1.7,} \mathrm{no's} 1$ \& 1a). The suspensory elements, cleithrum, anterior centra and all the caudal rays are ossified. Each olfactory cup is curled at the edges. The posterior margins of the hypurals are nearly vertical and are bordered by several poorly defined melanophores. 
There are 10 caudal rays and the anlages of 9 rays of the third dorsal fin. The melanic patches around the gut have increased in size and run together while an elongate melanic patch lies within the integument lateral to the cleithrum. A yellow tinge pervades the tissue around the otic capsules and the gut. Typically 17-20 melanophores run along the ventral mid-line although some individuals have as few as 14. One to 5 melanophores are present on the belly and a triangle of 3 straddles the cleithral symphasis. The neural pigmentation is unchanged.

Larva $12.5 \mathrm{~mm}$ s.1. All the rays and spines are visible in the fins except those of the first dorsal fin. The second dorsal fin is low and the membrane is continuous with that of the third. Each nasal tube is U-shaped while the head and abdomen are distinctly robust. A small tentacle sits above each eye. The pelvic rays extend two thirds of the length of the abdomen and all centra are ossified. The pectoral fin is proportionately laxge extending past the anus and the longest anal rays ile directly beneath the third dorsal fin.

Prejuvenile $14.3 \mathrm{~mm}$ s.l. The general features and relative proportions are very similar to the $13.5 \mathrm{~mm} \mathrm{s.1.} \mathrm{prejuveniles} \mathrm{of} \mathrm{N}$. compressus (Fig.16, no's $2,2 \mathrm{a} \& 2 \mathrm{~b})$. The tentacles are proportionately larger and the first dorsal fin is taller. The amount of pigmentation in the integument is not as extensive as that in N. compressus. A melanophore is present above the telencephalon, mesencephalon and the myelencephalon and about 1.1 more run along the spinal cord. Twenty pigment cells are associated with the rays of the anal fin and 3 medium sized ones are visible on the lateral surface of the pectoral lobe. Specimens are readily distinguished by meristic counts.

Subfamily Clininae

Tribe Clinini

Ericentrus rubrus (Hutton, 1872)

Adult diagnosis: (9 specimens)

Maximum S.I. $60 \mathrm{~mm}$; D. IV, XXXV-XXXVII, 1; A. II, 24-25; C. 11-12; P. 12; vertebrae 47-48; nasal tentacles 1 ; orbital tentacles 2; lateral Iine I 25; lateral line II 20; the two anterior spinous portions of the dorsal fin are poorly defined. 
Larval diagnosis: (20 specimens)

47-48 myomeres (17-18 pre-anal); gut elongate with dorsal profile interrupted by airbladder; born at about $10.0 \mathrm{mrn}$ S.L.; one melanophore on isthmus of throat and at anterior point of liver; prejuvenile at about $13.5 \mathrm{~mm}$ S.I.; meristic counts within range of adults; pre-anal vertebrae 16-17.

Spawning months: Specimens about $10.0 \mathrm{~mm} \mathrm{s.1.} \mathrm{are} \mathrm{present} \mathrm{in} \mathrm{the}$ plankton from July to February.

The adults of $\mathrm{E}$. rubrus are ovoviviparous (Moreland \& Dell, 1950). On 18.10 .70 a female (45 mm s.1.) gave birth to 22 larvae all about $10 \mathrm{~mm}$ s.1. In an aquarium at the Island Bay Marine Laboratory. Later larval and prejuvenile stages were collected from the plankton.

Larva $10.5 \mathrm{~mm} \mathrm{s.1.} \mathrm{(Fig.17,} \mathrm{no.2).} \mathrm{The} \mathrm{body} \mathrm{is} \mathrm{typically} \mathrm{long}$ and narrow, the suspensory elements are well defined and the lower jaw extends forward of the premaxillary. Prominent teeth are carried on the dentary and premaxillary and the edges of the olfactory cups are beginning to curl. The gut is long and coiled adjacent to the liver while a large characteristic airbladder interrupts the upper profile of the abdominal cavity. This aixbladder is typically larger and more clearly defined than those of the tripterygiids. The notochord is fully upturned, the posterior margins of the hypurals are vertical and 12-13 caudal rays are present. Several dorsal and ventral procurrent rays are also visible. The dorsal. and ventral fin folds are undamaged following birth, not having suffered the wear and tear of swimming or plankton nets. There are 47-48 myomeres 17-18 of which are pre-anal and 12 rudimentary spines are present in the dorsal fin fold near the caudal peduncle. No anal rays are visible. These larvae and those of Cologramus flavescens are characteristically without much pigmentation. Several xanthophores are scattered in the tissues around the otic capsules, beneath the brain and above the gut. Two melanic patches lie in the parietal peritoneum above the gut, one either side of the airbladder. A medium sized melanophore wraps around the anterior ventral corner of the liver and there are no neural pignent cells. Twentyone small melanophores run along the ventral mid-line closely associated with the anal rays.

By $11.0 \mathrm{~mm} \mathrm{s.1.} \mathrm{the} \mathrm{anal} \mathrm{sexies} \mathrm{has} \mathrm{increased} \mathrm{to} 26$ and there is a spindly elongate melanophore above the myelencephalon which sends branches over the cerebellum and onto the anterior spinal cord. 
Iarva $12.5 \mathrm{~mm} \mathrm{s.1.} \mathrm{The} \mathrm{pigmentation} \mathrm{has} \mathrm{not} \mathrm{altered} \mathrm{much} \mathrm{except}$ for the appearance of a small melanophore on the isthmus of the throat and two bordering the hypurals. The melanic patches in the peritoneum are joined above the airbladder. Two spines and 24 rays are present in the anal fir increasing in length posteriorly. Twenty-five to 26 dorsal spines are well defined, also increasing in size posteriorly, whereas the dorsal rays are barely visible. The depth of the tail beneath the posterior dorsal spines is greater and the dorsal and anal fins extend onto the caudal peduncle.

Prejuvenile $15.9 \mathrm{~mm}$ s.1. (Fig.17, no's 3 \& 3a). The anterior nostril is tubulax, a small tentacle sits above the eye and the gut is bunched particularly near the coil. The airbladder is still very prominent above the hindgut and the dorsal profile rises evenly to the $23 r d$ spine where lateral spine processes and associated muscles produce a more irregular contour down to the caudal peduncle. The contour above the anal fin is similarly irregular while the body depth is greatest beneath the 29th-32nd dorsal spines tapering from here to the narrow caudal peduncle. Anteriorly 4 dorsal spines rise higher than those immediately following marking the extent of the first dorsal fin. Each pelvic ray is curved and projects laterally from a point beneath the liver. Three to 4 small melanophores are visible above the posterior end of the spinal cord and one sits behind the articulation point of the jaw. The hypural pigment cells are frequently contracted to an axea between the plates and in some individuals a small melanophore is embedded in the muscle above the 5 th to last anal xay. Prejuveniles are present in the surge channel schools.

Juvenile $17.8 \mathrm{~mm} \mathrm{s.1}$. Three juveniles were shaken from the fronds of a brown alga Carpophyl. Iur sp. on 12.1.70. The overall proportions are similar to those of the adult although the body depth is proportionately less. Most of the integument is uniformly covered in tiny melanophores (brownish) which in some individuals are concentrated as 4 stripes running the length of the body from behind the eyes. Another row runs along the ventral surface from the lower jaw to the anus. The tiny melanophores in the integument are interspersed with xanthophores and erythrophores giving a golden-brown colour to the fish. 


\section{Cologramus flavescens (Hutton, 1872)}

\section{Di.agnosi.s:}

Summary compiled from Hutton (1872) and prejuveniles 15.0 and $19.0 \mathrm{~mm}$ S.I.; D. XXXVIII, 6-7; A, II 28-29; C. 12; $P_{I}$. 12; vertebrae 51-52; nasal tentacles none; orbital tentacles $1 ;$ the spinous dorsal fin is undivided.

\section{Larval diagnosis: (10 specimens)}

51-52 myomeres (17-18 pre-anal); gut elongate with dorsal profile interrupted by airbladder; born at about $10.0 \mathrm{~mm}$ S.L.; no melanophores on isthmus of throat or at anterior point of liver; prejuvenile at about $15.0 \mathrm{~mm}$ S.L.; meristic counts within range of adults; pre-anal vertebra

Spawning months: Larvae about $10.0 \mathrm{~mm} \mathrm{s.1.} \mathrm{are} \mathrm{present} \mathrm{in} \mathrm{the}$ plankton from late August to February.

I have found no adult specimens with developing eggs, but it is assumed that $\mathrm{C}$. flavescens is ovoviviparous like E. rubrus. The ear stages found in the plankton were no smaller than $10.5 \mathrm{mrn} \mathrm{s.l.} \mathrm{and} 1$ are nearly identical to those of E. rubrus.

Larva $10.5 \mathrm{~mm}$ s.l. (Fig.18, no's 1 \& la). The proportions a features are the same as those in equivalent stages of E. rubrus the airbladder is further forward in the abdominal cavity, the $n$ melanophores associated with the anal rays is higher $(22-23 \mathrm{cf}$. is an elongate melanophore above the myelencephalon and there 11 pigment cells along the spinal cord. No melanophore surrol antexior ventral corner of the liver and the melanic patches gut are joined over the airbladder. There are 51-52 myomere these are pre-anal.

Larva $15.1 \mathrm{~mm} \mathrm{s.1.} \mathrm{(Fig.18,} \mathrm{no's} 2 \& 2 \mathrm{a}$ ). Up to 14 run along the spinal cord including one close to the myel and 26 other pigment cells are associated with the anal J pigment cells lie beneath the last rays and spines of tr The nasal tube is $U$-shaped and the brain is narrow not of the anterior maxgins of the eyes. The airbladder $i$ anus and the coil in the gut is more pronounced. Thi visible in the dorsal fin which reaches its highest $\bar{c}$ ray ( 7 in all) then decreases posteriorly toward the 'Thirty rays are present in the anal fin increasing Equivalent sized specimens of E. rubrus appeax mor 
spine and ray compliments, well developed pelvic rays and precisely defined suspensory elements.

Prejuvenile $19.3 \mathrm{~mm}$ s.1. (Fig. 18, no's 3 \& 3a). The bony elements of the head are ossified particulaxly those of the suspensorium. The lower jaw with many small teeth extends forward of the premaxillary, also bearing teeth. There is a small tentacle above the eye, the airbladder is prominent and the long spatulate pectoral fin has a narrow base and thickened ventral rays. The spines in the first dorsal fin form a continuous series with the anteriormost ones undifferentiated. Approximately 18 interdigitated melanophores lie above the spinal cord including two close to the elongate pigment cell on the myelencephalon. A medium sized melanophore sits above the telencephalon. Each anal ray except the first has a small melanophore around its proximal end (29-30). Twenty-five pigment cells run along the mid-line beneath the last dorsal spines and rays. This series of melanophores is complete but for the anterior 3 which are separated from the rest by a space of two spines.

\section{Famjly Gobiesocidae}

\section{Trachelochismus melobesia Phillipps, 1927}

\section{Adult diagnosis: (Briggs, 1955)}

Maximum S.L. $31 \mathrm{~mm}$; D.10 (9-11); A. $8(7-8) ; P_{I} \cdot 23(22-24)$; C.12; vertebrae 31-33; nasal tentacle 1 simple.

\section{Larval diagnosis: (29 specimens)}

32-33 myomeres (16-18 pre-anal); up to 13 post-anal melanophores on ventral mid-line; $7.0 \mathrm{~mm} \mathrm{S.L.} \mathrm{notochord} \mathrm{upturned;} \mathrm{prejuvenile} \mathrm{at} \mathrm{about}$ $7.8 \mathrm{~mm}$ S.L.; meristic counts within range of adults; 14 pre-anal vertebrae; disc length about quarter standard length (prejuvenile).

Spawning months: Early September to late December.

The embryological and larval development of $T$. melobesia is described and figured by Ruck (1971). The larval development is also included here to supplement the description of rrachelochismus pinnulatus (Forster, in Bloch and Schneider, 1801). It is described and figured in greater detail, as further experience has allowed a better interpretation of structural features. 
Prolarva (Fig.19, no's 1 \& la). Prolarval length on hatching ranges from 4.8-5.5 mm (Ruck, 1971). The gut is long and extends to the base of the $16 \mathrm{th}-17 \mathrm{th}$ myomere. The number of pre-anal myomeres given by Ruck (1971) is lower (15) because myomere counts were taken from the cleithrum back excluding the two anterior ones. The upper peritoneum of the abdominal cavity is covered with numerous stellate melanophores, extending from above the yolk to the anus. Posterior to the anus are 15-17 myomeres. Stellate melanophores are present in the myomeres just past the anus, but their number is variable. An obvious yellow tinge surrounds the braincase, extending through the muscle above the gut to the 8 th myomere past the vent. There is no neural pigmentation.

Larva $6.3 \mathrm{~mm} \mathrm{s.1.} \mathrm{(Fig.19,} \mathrm{no.2).} \mathrm{The} \mathrm{yolk-sac} \mathrm{is} \mathrm{absorbed} \mathrm{and} \mathrm{the}$ gut reaches beyond the mid-length of the body. The oil globule, present within the yolk of the prolarva, is no longer visible. Pigmentation has altered very little except for the addition of 1-2 small melanophores to the inner surface of the pectoral base. The melanophores past the anus are not always as regular as those shown in the prolarva. The jaws are well formed, ossified and functional, although as yet without well defined teeth. Branchiostegal rays are faintly visible below the broad opercular flap. Rudimentary sucker buds lie ventral. to the heart and Iiver and the edges of the olfactory cup are curled inward but do not meet. The notochord is slightly bent upward and the caudal ray anlages are clearly defined just forward of the end of the notochord. The dorsal, ventral and caudal fin folds axe typically tattered, in paxticular the caudal fin. Below the dorsal and ventral fin folds are aggegations of granular tissue, particularly at the base of the future dorsal and anal fins.

Larva $7.0 \mathrm{~mm} \mathrm{s.1.} \mathrm{(Fig.19,} \mathrm{no.3).} \mathrm{The} \mathrm{nasal} \mathrm{tube} \mathrm{is} \mathrm{complete,}$ the brain is slightly broader particularly behind the eyes, the notochord is almost fully upturned and rudimentary hypurals carry 9 faintly ossified rays. These rays are directed into the lower part of the caudal lobe. Fine traces of supporting fibres radiate from the tip of the notochord into the caudal lobe. The condensation of tissue at the base of the dorsal and anal fins has increased. All the ryomeres are double chevronned and there is a small poorly defined airbladder high in the abdominal cavity above the livex. The pelvic sucker is broad with two well defined portions and the pectoral $\mathrm{fin}$ is lower on the body. The basic pattern of pigmentation is the same except for several stellate melanophores scattered along the 
belly. Most of the pigment cells along the ventral mid-line have migrated higher into the muscle and appear constant at 8-9 cells.

Prejuvenile $7.85 \mathrm{~mm}$ s.l. (Fig.19, no's $4 \& 4 \mathrm{a}$ ). The overall shape has altered slightly. Essentially there is a flattening and broadening of the head and an increase in the depth of the tail. The dorsal profile swings upwards just before the dorsal fin. In addjtion to the existing pigmentation numerous grey spheres lie scattered along the peripheral areas near the fins and over the dorsal integument of the head. The spheres are typical of many gobiesocid larvae and they occupy the sites of chromatophores which appear later in development as stellate entities. The urostyle is curved upwards but still extends a considexable distance into the caudal fin. The ventral sucker is well developed and functional because at this size specimens are able to cling to the sides of glass jars. All the rays are visible and ossified proximally. The lips are fleshy, the nostrils distinctly tubular and the lower jaws swing inward so that each articulation point is close to the ventral mid-line. About 9 melanophores remain on the belly concentrated near the anus and the uncoiled gut hangs slightly below the hypaxial flaps of muscle. A row of 8 evenly spaced, stellate melanophores runs along the lateral. surface above the anal fin while 4-5 smallex ones lie at the base of the anal rays. A yellow-green tinge pervades the tissue around the brajn and above the gut extending as far as the 8 th myomere past the anus. Two small melanophores are transversely situated just behind the myelencephalon.

Juvenile $8.7 \mathrm{~mm}$ s.l. (Eig.19, no's $5 \& 5 \mathrm{a}$ ). A single specimen was collected on 15.2.71 from beneath a tidepool stone. The overall features and proportions resemble those of the adult. The integument is almost completely covered by tiny spheres and stellate melanophores (brownish). The purple patch on the nape which is typical of the adult is absent in the juvenile. Peivic rays are visible extending postero-laterally from the anterior part of the sucker which has nearly all the sucker papillae present. The sucker is just over a quarter the standard length. 


\section{Trachelochismus pinnulatus (Forster, in Bloch and Schneider,} 1801)

\section{Adult diagnosis: (Briggs 1955)}

Maximum S.L. $71 \mathrm{~mm} ;$ D. 8 (7-9); A. $6(5-7) ; \mathrm{P}_{\mathrm{I}} \cdot 25(24-26)$;

C. 12 (11-12); vertebrae 31-33; nasal tentacles 1 spatulate.

\section{Larval diagnosis: (24 specimens)}

32-33 myomexes (19-21 pre-anal); no post-anal melanophores on ventral mid-line; $7.2 \mathrm{~mm}$ S.L. notochord upturned; prejuvenile at $9.3 \mathrm{~mm}$ S.L.; meristic counts within range of adults; 13-16 pre-anal vertebrae; sucker length fifth the standard length (prejuvenile).

Spawning months: Early July to mid October.

The embryological and prolarval development of T. pinnulatus is described and figured by Ruck (1973b). The larval development is very similar to that of T. melobesia with few features to separate them. T. pinnulatus is a largex clingfish than $T$. melobesia and at every level of development e.g. presence of rays, T. melobesia is consistently smaller.

Prolarva. Prolarval lengths at hatching range from 5.35-6.10 mm (ave. 5.7) (Ruck, 1973b), larger than those of T. melobesia $(4.80-5.50 \mathrm{~mm}$, ave. 5.4). The prolarvae of both species are identical except those of T. pinnulatus lack the melanophores along the mid-line past the anus and these are not added later in development.

Larva $6.6 \mathrm{~mm}$ 5.1. At this size the degree of development is the same as that in $6.3 \mathrm{~mm}$ larvae of T. melobesia (Fig.19, no.2). The airbladder is very prominant and is situated further back than it is in T. melobesia. There are 19-21 preanal myomeres compared with 16-17 in T. melobesia.

Larva $7.6 \mathrm{~mm} \mathrm{s.1.} \mathrm{This} \mathrm{larva} \mathrm{is} \mathrm{similar} \mathrm{to} \mathrm{the} 7.0 \mathrm{~mm}$ larva of T. melobesia (Fig.19, no.3), with an upturned uxostyle and 8-9 caudal rays. However, the sucker i.s not as well developed, consisting of two rounded lobes and the olfactory tubes are not yet complete.

Prejuvenile $9.6 \mathrm{~mm} \mathrm{s.1.} \mathrm{(F'ig.20a,} \mathrm{no's} \mathrm{1,} \mathrm{la} \mathrm{\&} \mathrm{1b).} \mathrm{The} \mathrm{level} \mathrm{of}$ development is roughly equivalent to that of $7.85 \mathrm{~mm}$ prejuveniles of T. melobesia. The body is, however, slightly more robust and there is no definite rise in the profile before the dorsal fin. The head is broad, the lips fleshy and the opercular flap is well defined. Many bones of the 
skull, all the rays and the centra are ossified. The airbladder causes a swelling of the gut cavity which raises 8 peritoneal melanophores above the rest. The lateral and dorsal integument of the head and peripheral zones of the body are covered in numerous tiny spheres. The fully functional sucker is a fifth the standard length and clearly defined but only a few papillae are present. Those on the posterior disc are the first formed and there are two small melanophores just behind the anterior disc.

\title{
Diplocrepis puniceus (Richardson, 1846)
}

\author{
Adult diagnosis: (Briggs, 1955) \\ Maximum S.L. $100 \mathrm{~min} ;$ D. 1.1 (10-11); A. $5(4-5) ; P_{I} \cdot 23(23-24)$; \\ C. 10; vertebrae 33 ; nasal tentacles 1 spatulate.
}

Larval diagnosis: ( 38 specimens)

32-34 myomeres (21-22 pre-anal); loose series of melanophores in lateral integument above anus; $7.7 \mathrm{~mm}$ S.L. notochord upturned; prejuvenilé at about $9.5 \mathrm{~mm}$ S.L.; meristic counts within range of adults; sucker length third standard length (prejuvenile).

Spawning months: Early July to late september.

The embryological and early larval development is described and figured by Ruck (1973b). For completeness the early larval development is also described and figured here.

Prolarva (Fig.20, no's 1 \& 2). Prolarval length on hatching ranges from $5.00 \mathrm{~mm}-6.05 \mathrm{mn}$ s.1. There is considerable variation in the amount of yolk present in each prolarva immediately after hatching. The yolk contains a single reduced oil globule. The heart is prominent and lies on the anterjor yolk nargin. The gut is long, and convoluted, and extended beyond the mid-length of the body. Numerous melanophores (30-50) are present on the myomeres of the body and tail, the first of which is placed behind the pectoral fin base; this series extends to the fifth myomere past the vent. Occasional specimens have as few as 6 melanophores on the myomeres. A yellow tinge is present deep in the muscle tissue directly beneath the melanophores of the body and tail. Numerous melanophores line the upper parietal peritoneum. The head is broad and blunt, and there are two clusters of stellate melanophoces beneath the myelencephalon. 
Larva $6.20 \mathrm{~mm} \mathrm{s.1.} \mathrm{(Fig.2,} \mathrm{no's} 3 \& 4$ ). The yolk is almost completely absorbed and the oil globule is very small. Pigmentation is unaltered except for the development of several melanophores at the base of each pectoral fin. A yellow tinge surrounds the base of the brain, particularly the myelencephalon, and extends posteriorly within the myomeres above the gut.

Larva $7.70 \mathrm{~mm} \mathrm{s.1.} \mathrm{(Fig.20,} \mathrm{no's} 5 \& 6$ ). The general shape of the larva has changed slightly. There is an increase in the depth of the tail and the relative size of the gut. The pigment pattern is unchanged and suckex buds are visible below the pectoral fin. Myomeres have the double chevron pattern and the notochord is slightly upturned in the tail. Six rudimentary rays are present in the caudal fin.

Larva $9.2 \mathrm{~mm} \mathrm{s.1.} \mathrm{(Fig.20a,} \mathrm{no's} \mathrm{2,} \mathrm{2a} \mathrm{\&} \mathrm{2b).} \mathrm{The} \mathrm{head} \mathrm{is} \mathrm{broad,}$ the tasl is deep and the heavily convoluted gut hangs below the hypaxial muscle flaps. The urostyle is upturned but continues into the caudal fin and the posterior margins of the hypurals are not yet vertical. The anal fin is short with 4 faintly ossified rays which lie close to the anus. The dorsal fin is almost twice as long as the anal fin and bears 10-11 rudimentary rays. Each of the 10 caudal rays is segmented and well ossified proximally. Pigmentation is unchanged and the sucker is functional but has very few papillae. The brain is broad, the nasal tube is complete, the lips are fleshy and the broad opercular flap is pointed at its posterior edge.

Prejuvenile $9.7 \mathrm{~mm} \mathrm{s.1.} \mathrm{(Fig.20a,} \mathrm{no's} 3 \& 3 a$ ). Considerable anatomical changes may take place with little increase in length. The dorsal profile of the head is counded whereas the ventral surface is quite flat. The jaws are horizontal and the anterior nostril is distinctly tubular. Dorsally the head is very broad and rounded anticipating the typical "horseshoe" shape of that in the adult. From the broadest point (opercular flap) the body tapers gradually toward the tail. The posterior half of the gut still hangs below the hypaxial muscle flaps and the dorsal and anal fins are clearly defined with well ossified rays. The posterior hypural margins are vertical and the caudal rays are even posteriorly. The sucker is well. developed and proportionately large at just under a third the standard length. Most of the papillae are present on both discs and arranged the same as those of the adult. The broadly spatulate pectoral fin is low on the body close to the sucker and the anal rays axe descendant. Pigmentation is unaltered except that the melanophores in the upper parietal 
peritoneur are more diffuse particularly in preserved specimens. The yellowgreen tinge still pervades the tissues around the brain, above the gut and beneath the lateral melanophores. Like T. melobesia and T. pinnulatus there are many tiny spheres over the entire integumentary surface and along the lower zones of the fin membranes. Those lateral to the trunk and tail appear concentrated on the myomeres outlining the chevron pattern.

Three larval and prejuvenile clingfish were collected near portobello (South Island, New Zealand) by Mr D. Robertson on 31.8.70. These are similar to the larvae and prejuveniles of D. puniceus except that the development is retarded comparatively and the pigmentation varies. Mexistically the two forms cannot be separated and the South Island series may be merely a subsp. of $D$. puniceus induced by different environmental conditions.

\section{Diplocrepis puniceus (South Island)}

Larva $10.4 \mathrm{~mm} \mathrm{s.1.} \mathrm{This} \mathrm{specimen} \mathrm{is} \mathrm{nearly} \mathrm{identical} \mathrm{to} \mathrm{the} 9.2 \mathrm{~mm}$ larva of D. puniceus (Wellington form) the only obvious difference being that of size.

Prejuvenile $11.35 \mathrm{~mm} \mathrm{s.l}$. All the rays are well defined and ossified proximally. The dorsal and anal membranes are continuous with the caudal fin, without any distinct constriction at the peduncle. The lateral pigmentation is similar to $\underline{D}$. puniceus but extends further forward beneath the pectoral fin and up onto the nape and anterior trunk muscles. These additional pigment cells are arranged in rows of about 10 melanophores. There are one to two melanophores on the pectoral base, two above the optic lobes and 4 above the myelencephalon. These neural chromatophores are absent in the Wellington variety.

Prejuvenile $12.3 \mathrm{~mm} \mathrm{s.1}$. The general shape resembles that of the $9.7 \mathrm{~mm}$ prejuvenile of $\mathrm{D}$. puniceus. Doxsally the head is rounded while ventrally it is flat, the body is deep above the anus and the gut is almost fully enclosed by the hypaxial muscle flaps. The dorsal and ventral fin membranes are constricted at the caudal peduncle. The dorsal rays are even whereas the anal rays are descendant. The sucker is about a quarter of the standard length and the lateral melanophores form a broad series extending from the fifth 
myomexe past the anus to the base of the pectoral fin. Anteriorly these pigment cells run onto the nape, trunk and ventrolateral aspect of the gut. Eight small melanophores are present on the pectoral base. Fifteen more lie above the optic lobes and 5 above the ryelencephalon forming no definite pattern.

\section{Gastroscyphus hectoris (Gunther, 1876)}

\section{Adult diagnosis: (Briggs, 1955)}

Maximum S.L. $43.6 \mathrm{~mm}$; D. $7(6-8)$; A. 7 (6-7); $\mathrm{P}_{\mathrm{I}} \cdot 2 \mathrm{I}(20-22)$; C. 8; vertebrae 43; nasal tentacles 1 broad; a slender clingfish with the posterior part of the body highly compressed.

\section{Larval diagnosis: (20 specimens)}

42-44 myomeres (25-27 pre-anal); $7.3 \mathrm{~mm}$ S.L. notochord upturned; about 18 interdigitated post-anal melanophores along the ventral mid-line; prejuvenile at about $9.50 \mathrm{~mm}$ S.L.; meristic counts within range of adults; 16-18 pre-anal vertebrae; sucker length sixth standard length (prejuvenile).

Spawning months: Mid June to early october.

On 20.11 .72 an adult female $G$. hectoris (35 mm s.1.) laid 15 eggs on the glass sides of an aquarium. The eggs were unfertilized but continued development to the blastodisc stage before dying. The chorion is sub-triangular ( $1.38 \mathrm{~mm}$ widest diameter) dorsally and dorso-ventxally flattened (Fig.21, no.la). The yolk is spherical (1.10 mm diameter), faintly yellow and contains one large oil globule and numerous smaller ones. An extensive search was made for other eggs among algal fronds frequented by the adults, but without success.

Larva $5.4 \mathrm{~mm}$.1. (Fig.21, no's $1 \& 1 \mathrm{~b}$ ). The head is small, quite blunt and the eyes interrupt the dorsal. profile. Elements of the jaws are poorly defined and the olfactory cups are uncurled. The pectoral fin is small and rounded situated above the incompletely absorbed yolk. Beneath. the yolk there is a small swelling of the future sucker. The gut is proportionately long extending over halfway down the body. The lobes of the brain are well defined (Fig.21, no.lb) and the notochord is straight. The dorsal profile is formed by translucent integument which is raised above the underlying myomeres and is continuous with the fin folds. Scattered over this integument and running along the base of the ventral fold are numerous 
tiny spheres. There are 42-43 myomeres, 25-27 of which are pre-anal. Many melanophores form a belt 2-3 cells wide in the upper parietal peritoneum, running from beneath the otic capsules to the anus. Along the ventral midline as far as the twelfth myomere past the anus are two rows of about 18 melanophores which are strongly interdigitated. There are 3-4 small, irregularly arranged pigrnent cells in the lateral integument near the anus.

Larva $7.3 \mathrm{~mm} \mathrm{s.1.} \mathrm{The} \mathrm{pigmentation} \mathrm{is} \mathrm{unchanged} \mathrm{and} \mathrm{the} \mathrm{head} \mathrm{and}$ trunk proportions are the same as at $5.4 \mathrm{~mm}$. The notochord is upturned in the tail but runs well into the caudal fin. The rudimentary hypurals carry 6 faintly ossified rays. There are no dorsal or anal rays present but there is a condensation of tissue at the point where they will form.

Prejuvenile $10.6 \mathrm{~mm} \mathrm{s.1}$. The general shape has altered considerably . The head is broad and low, the lips are fleshy, the nasal tubes are complete and the opercular flap is elongate rising gently to a point on the pectoral base. The gut extends over halfway and is propoxtionately large. The profile rises gently to the dorsal fin then slopes to the caudal fin. All the rays are ossified proximally and those of the caudal fin are faintly segmented. The membranes of the dorsal and anal fins are continuous with that of the caudal fin. Approximately 8 tiny melanophores run around the pericardial membrane and those above the gut form two main rows. One of the 4 melanophores near the anus has migrated forward a little and the head is covered by tiny spheres. There are many tiny, contracted exythrophores on the snout and on the muscle near the anus. These later spread over the entire body lying between the spheres. The sucker is functional but proportionately smaller than those of the previously described gobiesocid prejuveniles, being only a sixth the standard length. The anterior disc has no papillae but there are 6 melanophores on the isthmus between the anterior and posterior disc. Two rows of papillae are present on the posterior disc. Anterior and posterior to the sucker in the ventral integument is a medium sized stellate melanophore. The 8 pericardial pigment cells are visible through the anterior disc.

Juvenile $9.7 \mathrm{~mm} \mathrm{s.1.} \mathrm{(Fig.21,} \mathrm{no's} \mathrm{3,} \mathrm{3a} \mathrm{\&} \mathrm{3b).} \mathrm{A} \mathrm{single} \mathrm{specimen}$ was collected on the 8.10.71 from a brown alga at Island Bay. The body proportions resemble those of the adult. The tail is low and the anal fin has drawn away from the anus. Small stellate melanophores (fawn-brown) cover the entire body except the ventrolateral and ventral portions of the 
head, the fins and two patches along the lateral aspect of the trunk. The underlying tissue is tinged yellow-green. A curved bar composed of melanophores and exythrophores (maroon colour) runs from the premaxillary across the iris of the eye back to the ventral edge of the opercular flap. The entire skeleton is well ossified and the nasal tentacles are prominent.

Ten prejuvenile clingfishes collected by Mx M. D. Robertson at Portobello on 31.8 .70 were found to have overall features similar to the $10.6 \mathrm{~mm}$ prejuvenile of $\mathrm{G}$. hectoris. They do not have the interdigitated series of post-anal melanophores (at least in the preserved state) and ranged from 13-17 mm s.l. Meristically they are distinct from G. hectoris in having a lower vertebral count and a greater number of rays in the pectoral fin. This species is undescribed and has the following meristic counts:D.8 (7-9); A. 8 (7-9); C. 8; P I. 24-26; Vertebrae 39 (37-40).

\section{Gastrocyathus gracilis Briggs, 1955}

\section{Adult diagnosis: (Briggs, 1955)}

Maximum S.L. 31.0mm; D. 6 (5-6); A. $6(5-7) ; P_{I} \cdot 19$ (18-19); C. 8 ; vertebrae 42; nasal tentacles, 1 ; characterized by a sub-terminal mouth and an extremely slender tail.

Larval diagnosis: (20 specimens)

41-42 myomeres (25-26 pre-anal); slender larva; $6.2 \mathrm{~mm}$ S.L. notochord upturned; yellow-green tinge throughout myomeres of trunk and tail; 7-9 melanophores associated with spinal cord and notochord; prejuvenile at about $7.0 \mathrm{~mm}$ S.L.; meristic counts within range of adults; sucker length seventh standard length (prejuvenile).

Spawning months: Mid July to early October.

Larva $5.5 \mathrm{~mm}$ s.1. (Fig.22, no's 1 \& 1a). The larva at this stage is slender with an elongate gut extending over halfway along the body. All larvae in this series have a distinct yellow-green tinge throughout the body, more extensive and slightly darker than that found in T. melobesia or 1. pinnulatus. There are 41-42 myomeres $25-26$ of these are pre-anal. The head is small, rounded dorsally but flat ventrally. Each olfactory cup is uncurled, the otic capsules are round and the sucker is present directly beneath the pectoral fin as two raised pads of tissue. The notochord is straight with the caudal anlage beneath the posterior end. Arising anteriorly above the mid-gut the dorsal fin fold continues posteriorly into the caudal 
lobe which is in turn continuous with the ventral fin fold. The ventral fin fold is deeper than the dorsal. There is a condensation of tissue along the base of each fin fold particularly near the future fins. Numerous very tiny spheres are scattered over the peripheral zones of the trunk and tail. There are two rows of tiny stellate melanophores in the upper parietal peritoneum, running from beneath the otic capsules to the anus. The melanophores of the gobiesocid larvae seldom exceed $0.4 \mathrm{~mm}$ (medium size). In the integument lateral to the gut are 4-9 medium sized, spindly melanophores which continue ventrally as two rows of 4-6 pigment cells on the sucker bud. A single melanophore is present beneath the fleshy lobe of the pectoral fin. Gastrocyathus gracilis is an unusual gobiesocid in having up to 15 spinal melanophores. Two to 7 melanophores are present beneath the spinal cord above the anus and 2-3 may be visible on the horizontal septum between the epaxial and hypaxial muscles. A further 2-3 small melanophores lie close together on the dorsal mid-line just past the anus. The brain lobes are clearly defined in particular the mesencephalon which is very broad (Fig.22, no.1a). The chorioid of the eyes is iridescent green, overlain my many tiny stellate melanophores. The lenses are not visible dorsally as the eyes are directed downwards.

Larva $6.9 \mathrm{~mm}$ (Fig.22, no's 2 \& 2a). The lips are fleshy, the lower jaw is inferior, the edges of the olfactory cups are curled inward but not joined and each otic capsule is distinctly triangular. The notochord is upturned but continues beyond the hypuxals into the caudal fin. There axe approximately 10 rays in the caudal fin (the lower ones are obscure). The depth of the body is greatest level with the dorsal and anal fins and the gut extends well over halfway down the body. Anteriorly the gut is constricted by the developing liver and the slender airbladder. The sucker is well developed with clearly defined discs and very faint lateral. papillae. In typical gobiesocid fashion the lower jaw curves inward placing the points of articulation close to the ventral mid-line. Pigmentation including the green-yellow tinge is unchanged, except the melanophores on the belly have shifted to a point beneath the posterior sucker disc. Melanophores assocjated with the spinal cord form a more consistent pattern of 4-5 above and 3-4 below.

Prejuvenile $8.2 \mathrm{~mm} \mathrm{s.1.} \mathrm{(Fig.22,} \mathrm{no's} \mathrm{3,} \mathrm{3a \&} 3 \mathrm{~b}$ ). The body is elongate and slender although not as pointed in the snout or tapered in the 
tail as the adult. The head is broad behind the eye, rounded dorsally and flat ventrally. The upper jaw overlaps the lower, which bears long teeth, forming a subterminal mouth. Most of the skeletal elements are ossified particularly the suspensorium and the broad opercular flap extends well back onto the pectoral base. Each nasal tube is complete (U-shaped) but the anterior nostrils are without dermal tentacles. The membrane connecting the dorsal, anal and caudal fins is reduced and almost the entire gut is surrounded by the hypaxial muscle flaps which have joined along the ventral mid-line. The sucker is a seventh the standard length, is functional and has a pattern of papillae resembling that of the adult. Melanophores around the spine lie beneath the dorsal fin and some of these have migrated to positions level with the notochord. The iris of the eye is iridescent blue and the tinge in the tissues surrounding the brain is more yellow than that elsewhere in the body. A single melanophore sits on the ventral mid-line behind the anal fin. The integument of the body contains many tiny spheres which darken first on the head then along the dorsal and ventral surfaces and finally on the lateral aspects. Specimens this size were collected from surge channels at night using a light-pole and dipnet.

\section{Dellichthys morelandi Briggs, 1955}

\section{Adult diagnosis: (Briggs, 1955)}

Maximum S.L. $46.3 \mathrm{~mm} ;$ D. $9(8-9) ;$ A. 8 (8-9); P. $22(21-23)$; C. 11 (10-12); vertebrae 32; nasal tentacles 1 simple efongate; the adult is supecficially similar to that of $T$. melobesia but has a much more spatulate snout.

\section{Larval diagnosis: (10 specimens)}

32 myomeres (16-18 pre-anal); $6.7 \mathrm{~mm}$ S.L. notochord upturned; compact group of melanophores in lateral integument above anus; series of melanophores above brain and along dorsal mid-line; a melanophore on the ventrolateral aspect of myelencephalon; prejuvenile at about $7.8 \mathrm{~mm}$ S.L.; meristic counts within range of adults; sucker length fifth standard length (prejuvenile).

The larval development is compiled from 10 specimens collected between september and February 1971-72. The overall shape and size of the larvae at various stages of development is similar to that of T. melobesia. The pigmentation is, however, very different. The series is not figured. 
Larva $5.2 \mathrm{~mm} \mathrm{s.1}$. The head is narrow and blunt, the tail is slender and the elements of the jaws are poorly defined. The fin folds are continuous and deep with the dorsal one arising above the otic capsule, reaching its greatest height above the anus and decreasing gradually to the caudal peduncle. The caudal fin is spatulate with fine skeletal filaments and the notochord is straight. The gut extends halfway along the body appearing proportionately more robust than in other gobiesocid larvae. There are 4 medium sized melanophores above the optic lobes in line with 7 others irregularly spaced along the dorsal mid-1ine, at the base of the dorsal fin fold. A single small melanophore is present on the ventrolateral surface of the myelencephalon, visible laterally through the otic capsule. Within the upper parietal peritoneum are many interdigitated pigment cells which extend from beneath the otic capsules to the anus. This series covers the dorsolateral surface of the gut and appears as a uniform dark layer with individual cells poorly defined. Directly above the hindgut in the lateral integument is a compact rectangular series of 27-30 stellate melanophores forming a distinctive patch. One or two of the anterior pigment cells lie separate from the others and a little lower on the integument. There are also 1-2 small melanophores on the ventral midline past the anus. A yellow tinge permeates the tissues axound the hindbrain and the muscles above the gut. There are 32-33 myomeres 16-18 of which are pre-anal.

Larva $7.8 \mathrm{~mm} \mathrm{s.1.} \mathrm{The} \mathrm{head} \mathrm{is} \mathrm{low} \mathrm{with} \mathrm{the} \mathrm{ventral} \mathrm{surface} \mathrm{flattened.}$ The lips are fleshy, the nasal. tube is complete and the sucker is visible as two well defined discs which are without papillae. The notochord is upturned extending well into the caudal fin and the vertical hypural margins bear 7 faintly ossified rays. Anteriorly the dorsal fin fold is reduced arising abruptly above the anus then decreasing gradually towards the caudal fin. The broad anal fold runs from the anus to the caudal fin with a slight constriction at the peduncle. Anlages of 8 rays are visible as even series close to the muscle in the dorsal and anal fin folds. The gut extends over halfway along the body. There are up to 16 clearly defined melanophores above the gut, with an apparent reduction in the number compared with earlier stages. The rectangular series in the lateral integument is less compact having spread forward beneath the pectoral fin. In some individuals the melanophores along the dorsal midline are reduced in number to 1 or 2 cells above the brain. The yellow tinge is still present. 
Prejuvenile $8.5 \mathrm{~mm} \mathrm{s.1}$. The general shape from the lateral aspect is very similar to that of the $7.85 \mathrm{~mm}$ prejuvenile of T. melobesia except the head is more robust. Dorsally the head appears more narrow than that of T. melobesia accentuated by an elongate snout. The jaws are fleshy, the opercular flap is smooth and broad and there is a prominent airbladder above the posterior sucker disc. The sucker which is a fifth the standard length is fully functional with numerous papillae arranged like those in the adult. The gut tapers towards the anus and is not fully enclosed posteriorly by the hypaxial muscle flaps. Each fin membrane is long and continuous with that of the caudal fin which tapers posteriorly, in contrast with the flared caudal fin of $\mathrm{T}$. melobesia. There are two melanophores above the optic lobes and one above the myelencephalon. The original series along the dorsal mid-line is represented by 3 widely spaced pigment cells. Lateral melanophores are reduced in number to about 22, some of which curl around the ventral edge of the abdominal hypaxial muscle.

\section{Haplocylix littoreus (Forster, in Bloch and Schneider,} 1801)

Adult diagnosis: (Briggs, 1955)

Maximum S.L. $104 \mathrm{~mm}$; D. $6(5-6) ;$ A. $9(8-9) ; P_{I} \cdot 23(22-23)$; C. 8 ; vertebrae 41 ; nasal tentacles 1 .

\section{Larval diagnosis: (5 specimens)}

41 myomeres (26 pre-anal); integument of trunk and tail extensively covered with melanophores; several melanophores present above brain lobes.

Spawning months: June to eaxly December.

only 5 specimens were collected and these were all about $6 \mathrm{~mm} \mathrm{s.1.}$ The description is from preserved specimens.

Larva $6.3 \mathrm{~mm} \mathrm{s.1.} \mathrm{The} \mathrm{head} \mathrm{is} \mathrm{blunt,} \mathrm{dorsally} \mathrm{rounded} \mathrm{and} \mathrm{slightly}$ flattened ventrally. The gut extends over halfway along the body, the notochord is straight and a small portion of yolk remains antexior to the liver. Proportionately i.t is very similar to the $5.5 \mathrm{~mm}$ larva of G. gracilis, with the dorsal translucent integument lifted clear of the underlying muscle and continuous with the dorsal membrane. There are 41 myomeres, 26 of which are pre-anal. The trunk and tail are extensively covered by tiny 
stellate melanophores which continue anteriorly above the head and ventrally onto the anterior surface of the belly. The series ends abruptly about $1.0 \mathrm{~mm}$ from the end of the notochord. Dorsally the melanophores are concentrated in two rows, one each side of the fin fold. These continue forward onto the head as a mose scattered series. Similar rows run either side of the ventral fin fold. Several melanophores are scattered in the integument surrounding the muscular base of the pectoral fin.

\section{Discussion - larval development}

The larval and prejuvenile stages of 10 tripterygiid, 4 clinid and 9 gobiesocid species are described and illustrated in detail. Those described are: Tripterygiidae; Forsterygion varium (Forster in Bloch and Schneider, 1801), F. nigripenne (Valenciennes in Cuvier and Valenciennes, 1836), F. capito (Jenyns, 1842), Tripterygion segmentatum Mcculloch and Phillipps, 1923, Gilloblennius decemaigitatus (Clarke, 1879), G. tripennis (Forster in Bloch and Schneider, 1801), Helcogramma medium (Gunther, 1861?), two Helcograma species, new tripterygiid species (genus not certain): Clinidae; Notoclinus compressus (Hutton, 1872), N. fenestratus (Forster in Bloch and Schneider, 1801), Exicentrus rubrus (Hutton, 1872), Cologrammus flavescens (Hutton, 1872): Gobiesocidae; Trachelochismus melobesia Phillipps, 1927, T. pinnulatus (Forster in Bloch and Schneider, 1801), Diplocrepis puniceus (Richardson, 1846), Diplocrepis puniceus (South Island form), Gastroscyphus hectoris (Günther, 1876), Gastroscyphus species, Gastrocyathus gracilis Briggs, 1955, Dellichthys morelandi Briggs, 1955, Haplocylix 1ittoreus (Forster in Bloch and Schneider, I801). The tripterygiids were the most difficult to identify because over 20 allied species had to be considered before any positive larval identification could be made. Because of the small size of New Zealand and the relatively even coastal distribution of adult tripterygijas, the plankton taken from any one area could contain specimens of all the species. Variation in geographical distribution was of little help except where harvour varieties were involved e.g. F. nigripenne (marine and estuarine forms). The situation was furthex complicated by inadequate classification which meant dealing with undescribed species and in some cases scant meristic 
246.

data. The section on the clarification of adult nomenclature does not deal with any new tripterygiid species because these are at present being studied by Mr J. Moreland (National Museum). However, as much information as possible was obtained on these new species so that by a process of elimination the larvae of the described species could be defined with greater certainty. Several larval series, in addition to the 10 described here, were tentatively compiled but were not included because of persistant doubts about theix accuracy. These series cannot be completed with confidence until the adults have been described in detail, including their ranges of variation in meristic counts.

In the tripterygiids the early larval stages are virtually devoid of pigment, and that which is present is usually the same in related species, particularly of the genus Eorsterygion. Pigmentation patterns were therefore of minor diagnostic significance during early metamorphosis. Graham (1939) describes and illustrates the prolarva and early larvae of a species he regards as Tripterygion varium (egg and prolarval sizes suggest that the species was $\mathrm{F}$. capito) but the work is of 1 imited diagnostic value in that 
and by less objective factors such as the shape of the snout and olfactory capsule, fin profiles etc. (see discussion on larval skeleton). The above relationship of adult and larval size is demonstrated by comparing F. varium and $\mathrm{F}$. nigripenne with $\mathrm{F}$. capito, the last named of which is much smaller as an adult than the former two. In F. capito the general pigmentation of the head and tail takes place at about 20-22 mm s.1. compared with about $28 \mathrm{~mm}$ for $\mathrm{F}$. varium and about $26 \mathrm{~mm}$ for F. nigripenne. This size shift is apparent even in the very early stages where the average standard lengths of the prolarvae of $F$. varium and $F$. nigripenne are $5.85 \mathrm{~mm}$ and $5.95 \mathrm{~mm}$ respectively compared with $4.9 \mathrm{~mm}$ for those of F. capito (Ruck, 1973a, in press a). A size shift is also apparent between the larvae and prejuveniles of F. varium and F. nigripenne (maximum adult size for both Ino mu s.1.) although less marked and at a later stage of development. At about $26 \mathrm{~mm}$ s.1. prejuveniles of F. nigripenne are stocky and have the full spine and ray complement of the adult. Equivalent sizes of $E$. varium have no first dorsal fin developed and appear more slender. The clinids share a similar size relationship to that found in the tripterygiids. Notoclinus fenestratus is larger than $\mathbb{N}$. compressus and its larvae are laxger than those of $\mathrm{N}$. compressus at any given level of development. The clinids also exhibit the marked sequential development of rays, spines and vertebrae and hence developmental stages may be compared in much the same way as in the tripterygiids. The gobiesocids have single short dorsal and anal fins, the elements of which arise simultaneously and hence are not so important in gauging developmental stages. Nevertheless size relationships between adults and larvae have significance when dealing with related species. Trachelochismus pinnulatus is a much larger clingfjos than $\mathrm{T}$. melobesia and at every level of development e.g. appearance of suckers and rays, I. melobesia is consistenty sraallex.

The size of the larvae when fins and other features appear, and the size of the larvae when they transform into juveniles, has been used by other researchers to separate closely related larval fishes (Ahlstrom and Bal1, 1954; Ahlstrom and Counts, 1958; Berry, 1959; Norden, 1961; Richards and McBean, 1966; Benzie, 1968d; Mosex and Ahlstrom, 1970, 1972, 1974; Moser, 1972). What has not been emphasized is the significance of larval. size in relation to adult size (see discussion above). An appreciation of the relationship between adults and larvae was of considerable value in the initial sorting of larval series. The young tripterygiids were extremely 
abundant in the inshore plankton samples and presented a bewildering array. Subtle diagnostic characteristics were not immediately apparent. However, it was possible to extract from the samples specimens at given stages of development, and to align these with earliex and later stages, until complete series covering the whole range of larval length were formed. Specimens too large or too small to 'fit' the sexies were rejected. Once the specimens were aligned in this manner, more standard diagnostic features were used to confirm the series. Even with similar sized larvae of different species this technique highlighted differences in pigmentation etc., by isolating groups of species from the many other tripterygiids and clinids also present in the sample.

An understanding of the relative sizes of larvae may possibly be used to predict the size of adults for those larval series which have not been matched with any adult species (described previously or not) e.g. the two Helcogramma species, unknown tripterygiid species (genus unknown), Gastroscyphus species and the South Island form of D. puniceus. As an example the $9.7 \mathrm{~mm} \mathrm{~s} .1$. prejuvenile (full ray complement just formed) of $\mathrm{D}$. puniceus found in coastal waters near wellington is much smaller than its South Island counterpart at $12.3 \mathrm{~mm}$ s.1. (specimen fxom Portobello). If developmental trends are consistent with those described earlier then a much larger adult for the South Island form would be anticipated, and one presunably not present at high tide levels where it would surely have readily been detected.

prejuvenile tripterygiids were distinguished using the full range of adult meristic data (whexe available), pigmentation, and minor characters such as nasal and orbital tentacles and fin shapes.

The New Zealand clinids and gobiesocids, being fewer in number and fairly well documented as adults, were a lessex problem. The genera are unique to New Zealand, within most cases single species and no known close relatives (Hutton, 1872; Gill, 1893; Briggs, 1955). Clinid and gobiesocid larvae from unrelated adults were readily distinguished by a wide range of characteristics (Ahlstrom, 1975) although differences in size at given stages of development were also useful. With respect to the latter, the relative scarcity of gobiesocid larvae in plankton tow samples tended to be a hindxance. This was overcome by the use of light poles and dipnets which greatly increased the number of larvae and prejuveniles collected.

Morphometrics, while stressed in some larval studies (Berry, 1959; Noxden, 1961; Runyan, 1961; Moser, 1972; Aboussouan, 1975; Ahlstrom, 1975), 
were not used extensively to separate closely related tripterygiid, clinid and gobiesocid larvae, except for standard length as related to stages of development. Variations in snout to vent distance, head width and head length (see appendix) were of some significance in the tripterygiids and clinids but mainly for the separation of larvae into genera. Regression graphs were not required for this. Notoclinus larvae and prejuveniles, for example, typically have a shorter snout to vent distance than in any of the other tripterygiids and clinids. This is reflected in the low preanal myomere count of 9-10 compared with over 10 for the others. Snout to vent, head width, and head length when expressed as percentages of standard length did not vary enough between closely related larvae to be of diagnostic value. However, in the gobiesocids the dimensions of head depth and sucker disc length were useful (Runyan, 1961).

New Zealand literature dealing with the larval. stages of tripterygiids, clinids and gobiesocids, although limited, has been of some value in identifying common species. Regan (1914-16) illustrates a late larval stage of T. varium? and Elder (1966) describes and illustrates incomplete larval and prejuvenile series of G. decendigitatus, N. compressus, D. morelandi, T. melobesia and T. pinnulatus. Elder's illustrations are all from preserved specimens and therefore important diagnostic patterns are not recorded, with diagnosis relying on rays, spines and vertebrae. This is possibly one of the reasons why Elder has not described many of the early larval stages where rays etc are not well developed. Daxby (1966) gives a brief description of the prolarval stage of H. medium (Gunther, 1861), but because of confusion in the nomenclature (see clarification of adult nomenclature and emoryological. discussion) it is not clear which aduJ.t species is involved. Graham (1939, 1953) in addition to a description of the early larvae of T. varium?, describes the early larvae of D. puniceus. His descriptions are very brief and are insufficient to separate D. puniceus from the superficially similar larvae of the acanthoclinid Acanthoclinus quadridactylus (B1och and Schneider, 1801) (Graham, 1939, 1953; Jillet, 1961, 1968). Diplocrepis puniceus may be distinguished from A. quadridactylus in having fewer melanophores high in the lateral integument above the gut, no well developed median fin ventral to the gut, and no coiled gut (coiled early in A. quadridactylus) at any time during the early metamorphosis. Coakley (1964) describes the prolarva of T. pinnulatus and Robexts (1968) presents a valuable photograph of a larval Gastrocymba quadriradiata (Rendahl, 1925). Roberts does not describe G. quadriradiata 
in any detail, but the photograph illustrates a pigmentation pattern that resembles that of $\mathrm{H}$. littoreus. No specimens of G. quadriradiata were found near Wellington and this is not surprising as no adults have been recoxded noxth of the Auckland Islands (Briggs, 1955). Moreland and Dell (1950) report on the ovoviviparity of E. xubrus but no confirmation of the same in $\mathrm{C}$. flavescens has been recorded in the literature. Cologrammus flavescens is a member of the Clininae, a subfamily well known for its ovoviviparity (Hubbs, 1.952; Penrith, 1969). As its larval development is very similar to $\mathrm{E}$. rubrus and no larvae smaller than $10.5 \mathrm{~mm}$ were recorded from the plankton ( $10.5 \mathrm{~mm}$ is the size of E. rubrus larvae when born), it is assumed that $C$. flavescens is also ovoviviparous.

The prolarvae of some of the tripterygiids, clinids and gobiesocids were identified after they had hatched from eggs collected from the shore (see section on embryological development). This assisted greatly in the identification of later larval stages because the prolarvae are well. developed at hatching and have some featuxes that are stable enough to link them with later stages. For example G. decemdigitatus, G. tripennis and D. puniceus have diagnostic and persistent pigmentation patterns laid down during late enbryonic stages. Prolarvae of the Eorsterygion species, however, are virtually identical and were, therefore, of limited value when identifying more advanced larvae.

Emphasis has been given recently to the value of larval characters as aids in differentiating taxa (Bertelsen, 1951; Ege, 1953, 1957; Orton, 1963; Castle,1969) and in defining evolutionary lineages (Orton, 1953b; Moser and Ahlstrom, 1970, 1972, 1974). "Both 1arvae and adults show variability, species differences, and group resemblances," (Orton, 1953b). A comparison of the larval stages of the New Zealand tripterygiids, clinids and gobiesocids for the most part affixms the existing taxonomy and reflects a little on phylogenetic origins. The larval development of $\mathrm{F}$. varium and $\mathrm{F}$. nigripenne is very similar. The larvae of $\mathrm{F}$. capito were not described because of meristic similarity between them and those of other undescribed species such as the "yellow and black blenny" and the "oblique swimming blenny". There is an abundance of a certain type of larva in the plankton which although similar in shape to larvae of $F$. varium and F. nigripenne is much smaller throughout development. It is assumed that these larvae, because of their abundance and their myomere counts between 43 and 45 , are the early stages of $E$ - capito and the two undescribed species. 
These species cannot be separated using the principle of size relationship between larvae and adults because the adults of all three species are of comparable size. On this basis the larval series of each would be expected to have similar dimensions at comparable stages of development. Forsterygion capito, the "yellow and black blenny" and the "oblique-swimming blenny" need careful taxonomic study so that a wider range of diagnostic characters can be compiled for use in larval classification. Except for size differences the prolarvae of all three described Forsterygion species are identical (Ruck, 1973a, in press a). The strong resemblance between the early stages of F. varium, F. nigripenne and F. capito indicates a close systematic relationship and reinforces the decision to remove capito from Tripterygion.

The larvae of Gilloblennius decemdigitatus and G. tripennis, while superficially similar in shape, have markedly different pigment patterns. This supports the earlier contention dexived from embryological data that these two species are quite divergent. Analysis of their adult osteology etc., may see the formation of a separate genus for decemdigitatus. The present study has shown that larvae are of value in detecting the presence of unrecognised species. Moser and Ahlstrom (1974) described the larvae of 11 myctophids of the genus Hygophum, only 9 of which were currently known from the adults. This prompted a closer look at the adults with subsequent discovery of the two undescribed ones. In the present study at least three unknown species of tripterygiids, and possibly two of gobiesocids, have been found in their larval phases. Finding the adults of these may not be easy, as early stages of fish may travel great distances from their sites of original incubation. Also the tripterygiids and gobiesocids are generally secretive, weed and crevice-dwelling animals which are often difficult to collect using conventional methods. By contrast planktonic enviroment is nore uniform and in some ways easier to sample than the tidal zone. This, in part, may explain why new species are revealed in larval studies. Another reason is that larvae of some fish taxa exhibit greater interspecific differences than do the adults. For example myctophids of the genus Hygophum, are very difficult to identify as juveniles and adults, whereas their larvae are highly distinctive and easily separated (Moser and Ahlstrom, 1974). At first sight this contrasts with the general rule that closely related adults (meristically similar etc.) usually have similar ard difficult to separate larvae, such as the 
cyprinids (Balinsky, 1948), clupeids and engraulids (Dakin and Colefax, 1934, 1940; Mansueti and Hardy, 1967; Baker, 1972, 1973), ictaluxj.ds (Mansueti and Hardy, 1967), percids (Norden, 1961), carangids (Berxy, 1959), scorpaenids (Morris, 1956; Moser, 1967, 1972), gobiesocids (Runyan, 1961) etc, etc. However, the larvae of Hygophum can be separated into "...three highly distinct subgeneric groups..." (Moser and Ahlstrom, 1974), suggesting that while the adults are superficially similar they are in fact phylogenetically divergent, and in agreement with this the larvae are dissimilar also.

The abundance of larvae in an area, and in particular prejuveniles, is an indication of the proximity of the adults, as the further the young travel the more dispersed and less abundant they will be. The three Helcogramma species treated here (two with undescribed adults) are present in the surge channels around the Wellington coast, suggesting that the three adults are also present locally. It is likely that these adults have alxeady been collected from the Wellington shore, and that close examination of museum collections will reveal theix presence.

Notoclinus fenestratus and $\mathrm{N}$. compxessus have been confused in the literature, but consistent larval and adult differences now establish them as separate species. The larvae are similar to those of the tripterygiids although they are genexally more robust, smallex and have tightly coiled guts early in development. The prejuveniles and adults have the dorsal fin divided into three portions moxe typical of the tripterygiids than of the clinids (Clininae). Notoclinus fenestratus and $N$. compressus are oviporous, typical of other members of the Labrisominae (Hubbs, 1952; Penrith,1969). Notoclinus fenestratus and $\mathrm{N}$. compressus are closely related sharing a very similar larval and prejuvenile development. They have melanic patches on the anterolateral peritoneum of the abdominal cavity, which are not generally found in the tripterygiids or the clinids such as E. rubrus and C. flavescens. Larval and adult characteristj.cs of $\mathrm{N}$. fenestratus and $\mathrm{N}$. compressus are in some respects closer to those of the tripterygiids than the live-bearing clinids E. rubrus and C. flavescens. The larvae of the latter two species are almost identical when first born but quickly diverge during later development. This divexgence is essentially one of size although there are differences in the shape of the dorsal fins. The larvae of $\mathrm{E}$. rubrus mature more rapidly than those of c. flavescens and, thexefore, acquire their full spine and ray complements at a smaller size $(15.9 \mathrm{~mm}$ s.1. c.f. $19.3 \mathrm{~mm}$ s.1.). Exicentrus rubrus and C. flavescens have unusually prominent air- 
bladders which set them apart from N. compressus, N. fenestratus and the tripterygiid species where the airbladders are reduced, and often not visible at all.

In retrospect, therefore, within the New Zealand Tripterygiidae there are three main groups of species represented by the following genera; Forsterygion, Gilloblennius and Helcogramma. A fourth smaller group contains Tripterygion. Only the prejuvenile stages of $\mathrm{T}$. segmentatum have been described. It is possible that these four groups represent approximately the original number of tripterygiids isolated in New zealand waters, and that the large number of related species now present have resulted from relatively recent speciation. The New zealand tripterygiids are considexed primitive (Springer, pers. comm.) and have presumably been maintained in the isolated waters of New Zealand in much the same way as the gobiesocids have (Briggs, 1955). This number of groups may increase slightly as more of the 20 or so species of tripterygiids are described, indicating the presence of moxe genera.

The New Zealand clinids appear derived from four essentially unrelated forms i.e. Notoclinus, Ericentrus, Cologrammus and Cristiceps (no larvae of Cristiceps found). The characteristics of the adults (see clarification of adult nomenclature) of Exicentrus and Cologramus are more distinct than those of the larvae. Notoclinus fenestratus and N. compressus, because of strong similarities expressed in the larvae as well as in the adults, are probably the result of recent speciation.

The larval gobiesocids are, with the exception of Trachelochismus pinnulatus and T. melobesia, easily identified as distinct series which differ in a number of characteristics. The larvae, therefore, strongly support the proposal that New zealand gobiesocids are essentially relict anà unrelated (Briggs, 1955). Trachelochismus pinnulatus and T. melobesia are closely related, as confirmed by their larvae which differ only in size and in the number of post-anal melanophores. The larval development of Dellichthys morelandi, in terms of relative size and shape of larvae, is similar to that of T. melobesia and supports the inclusion of these two relict genera within the subfamily Trachelochisminae (Briggs, 1955).

Considexing the length of time that the New Zealand gobiesocids have been isolated from the original Western Pacific stock (Briggs, 1955), it is interesting to ask whether further speciation has occurred in the New zealand area. A survey of the known adult New Zealand clingfishes gives little evidence of recent speciation, apart from that of Trachelochismus 
into T. melobesia and T. pinnulatus. This is perhaps surprising considering the number of species present here which are maintained by isolation in areas free from competition and in an environment that would favour speciation i.e. extensive coastline and islands separated by deep water. A study of the larvae suggests that some speciation may have occurred and that the present knowledge of adult clingfishes is inadequate to show this fully. This study has demonstrated that in New Zealand waters there are two previously unrecorded subspecies or species of gobiesocids (at least as larvae and prejuveniles) belonging to the genera Diplocrepis and Gastroscyphus. More extensive collections of adult clingfishes will undoubtedly reveal the unrecognized taxa. The larvae of D. puniceus and those of the southern subspecies, which differ only in melanophore pattern and relative sizes (vertebral and ray counts identical), may represent forms in the process of speciation. This divergence is possibly being maintained by limited geographical isolation between the waters of the North Island and South Island of New Zealand. It is also possible that this initial divergence is more strongly reflected in the larval and prejuvenile stages than in the adults, especially as characteristics such as size and pigmentation are less diagnostic in the adult fishes. The new species of Gastroscyphus is meristically readily separated from G. hectoris, but is morphologically similar enough to suggest that the two have only recently been dexived from a common ancestor.

\section{An approach to larval studies}

The identification of the early stages of 23 New Zealand tripterygiids, clinids and gobiesocids is in itself evidence that the aims of this thesis have been fulfilled. This has meant the application of currently accepted and modified techniques for the identification of larval fishes, as have been described earlier in the section on prolarvae, larvae and prejuveniles. This work stresses the importance of understanding the fishes being studied on as broad a basis as possible, including a consideration of methods of collection and preservation, rearing of young stages (eggs), osteology, terminology for intraspecific and interspecific comparison, and adult taxonomy etc. Development in its broadest sense begins at fertilization and ends at death and therefore the parameters defining embryology, larval development and adulthood are artificial divisions of a broad spectrum of change. For 
example the bony skeleton changes throughout the life of the fish, and does not attain i.ts final form simply at maturity. Mansueti and Hardy (1967) "...feel that other aspects of the biology of species involved are of direct or potential value in the collection and identification of eggs, larvae and juveniles." Runyan (1961), Moser (1972), Ahlstrom (1975) indicate directly or indirectly the idea that diagnostic characters may be drawn from many aspects of a fish's life.

Ahlstrom (1975) stresses "...that one must know basic information about the adults of a region before ichthyoplankton identification can be made." This includes an understanding of the adult nomenclature. With the classification of the New Zealand tripterygiids clarified it would be a relatively simple matter to identify the larvae of the remaining species. The techniques have been developed for identifying the tripterygiid larvae and they may be applied provided more information is available on the adults i.e. precise ranges of meristic data. Larval studies, as stated earlier, may be instrumental in the detection of new species and in the reinforcement of curcent adult nomenclature.

A study of the larval and adult osteology may establish the point when certain meristic complements are complete, define various developmental stages for inter-specific comparison and provide knowledge about the overall larval and adult morphology (shape, size etc.). A poor understanding of the latter is reflected in the relatively low quality of technical drawings found in many developmental papers. Accurate diagrams showing appreciation of overall. structure are to be found in works by Morris (1951), Berry (1959), Mansueti (1962, 1964), Mansueti A. J. (1963, 1964) and Orton (1963). An equal understanding of anatomical terminology is also important when documenting changes in structures during metamorphosis.

Many diagnostic details are missed by researchers who use preserved specimens only, In these, pigmentation patterns are faded and obscured by opaque flesh. Nearly all the illustrations and descriptions in this thesis were made from either living (anaesthetized) or freshly preserved specimens which had spent no more than a few days in 5\% formalin. A further advantage in using "fresh" specimens is that they are often less distorted by shrinkage and give a more accurate picture of morphology than those preserved for many weeks. It is realized that not all studies lend themselves to the use of 'fresh' specimens, where plankton sorting cannot be immediate, and if so then perhaps diagnosis using temporary characters 
is of little value. However, when dealing with similar species and where all available features must be considered then some sort of clearing process ( $\mathrm{KOH}$, glycerine etc) should be used to reveal vestiges of deep melanophores etc.

If fresh specimens are to be provided then attention must be given to efficient methods of collecting the larvae. Furthermore without adequate samples larval series will be incomplete. Several methods for collecting larvae involving plankton nets, dipnets, light-traps and "slurp-guns" were adapted for use in this study.

It has been shown that a considerable proportion of the early life of many inshore fishes is spent within the tidal zones (eggs) and in the surge channels (laxvae and prejuveniles). This emphasizes the need for care of the rocky shoreline which now receives considerable abuse in terms of sewage, reclamation, and general human activity.

Artificial key to larvae and prejuveniles of species described in this study. Prejuvenile characteristics are used only where features are obscured during latex development.

\section{TRIPTERYGIIDAE AND CIINIDAE}

1. (8) gut elongate duxing early stages, 2 widely spaced melanic patches in the upper peritoneum, 14-18 pre-anal myomeres;

2 (5) airbladder small and does not disrupt the upper profile of the abdominal cavity:

FORSTERYGION Whitley and Rhillipps, 1940

3 (4) 48-51 myomeres, prejuveniles 23-24 spines in second dorsal fin:

Forsterygion varium (Forster, in Bloch and Schneider, 1801)

4 (3) 45-47 myomeres; prejuveniles 13-15 rays in thixd dorsal fin, 1-2 spines and $25-27$ rays in anal fin:

Forstexygion nigripenne (marine form) (Valenciennes in Cuvier and Valenciennes, 1836)

5 (2) airbladder prominent, disrupting upper profile of the abdominal cavity;

6 (7) 47-48 myomeres, 1 melanophore at apex of liver and 1 on isthmus of throat: 
Ericentrus rubrus (Hutton, 1872)

7 (6) 51-52 myomeres, no melanophores at apex of liver or on isthmus of throat:

\section{Cologrammus flavescens (Hutton, 1872)}

8 (1) gut bunched throughout development, 9-13 pre-anal myomeres;

9 (14) melanic patches above gut separate during early development, 3 melanophores on isthrmus of throat, 11-13 pre-anal myomeres:

HELCOGRAMMA MCCulloch and Waite, 1918

10 (13) series of melanophores and exythrophores along belly, 7-8 xanthophores around posterior end of notochord, 11 (11-12) pre-anal myomeres;

11 (12) 5 melanophores beneath caudal peduncle, melanophore above mesencephalon not central in larvae, prejuveniles 2 spines 20-21 rays in anal. fin, no melanophore above telencephalon:

Helcogramma medium (in part Gunther, 1861)

12 (11) 7 melanophores beneath caudal peäuncle, melanophore above mesencephalon central, I melanophore above telencephalon, prejuveniles no spines in anal fin, first dorsal spines descendant:

\section{Helcogramina sp. 2}

13 (10) no pigment cells along belly, no xanthophores around notochord, 13 (12-13) pre-anal myomeres, prejuveniles 2 spines 21-23 rays in anal fin:

\section{Helcogramma sp. 1}

14 (9) melanic patches above gut confluent throughout development, 1-3 melanophores on isthmus of throat, 9-12 pre-anal myomeres;

15 (16) 2 prominent rows of melanophores on belly, broad band of erythrophores in integument of trunk, 8 melanophores on isthmus of throat:

\section{New tripterygiid species}

16 (15) melanophores on belly do not form distinct rows, no band of erythrophores on trunk;

17 (20) 1 melanophore on each anterolateral wall of abdominal cavity, 9-10 pre-anal myomeres, 3 melanophores on isthmus of throat: 
NOTOCLINUS Gill, 1893

18 (19) 38-40 myomeres, I melanophore above telencephalon in larvae larger than $8.5 \mathrm{~mm}$, prejuveniles 12-13 rays in third dorsal fin:

Notoclinus fenestratus (Forster, in Bloch and Schneider, 1801)

19 (18) 35-36 myomeres, no melanophores above telencephalon, prejuveniles 9-11 rays in third dorsal fin:

Notoclinus compressus (Hutton, 1872)

20 (17) no melanophores on the anterolateral walls of abdominal cavity, 11 (10-12) pre-anal. myomeres, I melanophore on isthmus of throat, 1 melanophore on caudal anlage or between hypurals:

GILIOBLENNIUS Whitley and Phillipps, 1940

21. (22) 42-44 myomeres, 3-5 large, elongate melanophores along dorsal and ventral mid-lines associated with rows of tiny erythrophores:

Gilloblennius decemdigitatus (Clarke, 1879)

22 (21) 38-40 myomeres, I-2 elongate melanophores along mid-line above caudal peduncle, prejuveniles 13-16 rays in third dorsai fin:

Gilloblennius tripennis (Forster, in Bloch and Schneider, 1801)

\section{GOBIESOCIDAE}

1 (8) lateral integument covered with numerous melanophores;

2 (3) trunk and tail extensively covered with tiny melanophores, 26 pre-anal myomeres:

Haplocylix littoreus (Forster, in Bloch and Schneider, 1801)

3 (2) melanophores restricted to head and trunk, 16-22 pre-anal myomeres.

4 (5) 16-18 pre-anal myomeres, early larvae lateral melanophores compact group near anus, melanophores above brain and along dorsal mid-line:

$$
\text { Dellichthys morelandi Briggs, } 1955
$$

5 (4) 21-22 pre-anal myomeres, lateral melanophores evenly spaced:

$$
\text { DIPLOCREPIS Gunther, } 1861
$$

6 (7) lateral melanophores do not extend onto nape or dorsurn of trunk, no neural melanophores: 


\section{Diplocrepis puniceus (Richardson, 1846)}

7 (6) melanophores extend onto nape and dorsum of trunk, up to 20 melanophores above mesencephalon and myelencephalon (prejuveniles): Diplocrepis puniceus (South Island)

8 (1) no melanophores in lateral integument;

9 (12) a series of melanophores along the ventral mid-line past the anus;

10 (11) 16-17 pre-anal myomeres, up to 13 separate cells in post-anal. series:

Trachelochismus melobesia Phillipps, 1927

11 (J.0) 25-27 pre-anal myomeres, about 18 interdigitated cells in postanal series:

\section{Gastroscyphus hectoris (Gunther, 1876)}

12 (9) no melanophores along the ventral mid-line past the anus;

13 (14) 25-26 pre-anal myomeres, dark yellow-green tinge throughout muscle tissue of body, spinal melanophores:

\section{Gastrocyathus gracilis Briggs, 1955}

14 (13) 19-21 pre-anal myomeres, yellow-green tinge only above gut and around brain, no spinal melanophores:

Trachelochismus pinnulatus (Forstex, in Bloch and Schneider, 1801) 
Fig.1. Forsterygion varium. No's 1 \& la: $11.5 \mathrm{~mm}$ larva; 2: $14.9 \mathrm{~mm}$ larva; $3,3 a \& 3 b: 22.2 \mathrm{~mm}$ larva.

Abbreviations: $A B$, airbladder; $A n R$, anal rays; $A n s$, anal spines; An $(x)$ rudimentary anal rays; BR, branchiostegals; CaR, caudal fin rays; $C a(r)$, rudimentary caudal rays; $C T$, coil in gut; CIM, melanophores at cleithral symphasis; Clp, cleithral pigment; LClM, lateral pigment on cleithrum; Cor, cornea; DM, pigment on dorsal midline; GM, melanophores above gut; Hy, hypurals; Ir, iris; L, liver pigment; MM, pigment above mesencephalon; MyM, pigment above myelencephalon; mx, maxillary; ner, neurocranium; NaT, nasal tentacle; ObT, orbital tentacle; Oca,

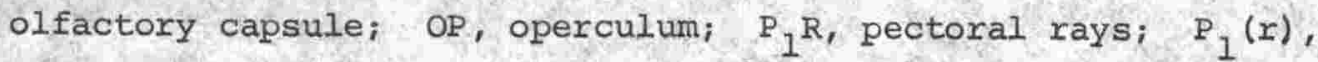
pectoral ray rudiments; $P_{2}$, pelvic rays; $P_{2}(x)$, pelvic ray rudiments; pmx, premaxillary; POP, preoperculum; pst, pineal stalk; SCC, semicircular canal; SM, spinal pigmentation; TM, pigment above the telencephalon; TaM, pigment bordering the hypurals; $U$, urostyle; VM, pigment along the ventral midine; Xa, xanthophores; lst D, first dorsal fin; 2nd D, second dorsal fin; 3rd D, third dorsal fin; 2nd DS, second dorsal spine; 3rd DR, third dorsal rays. 





Fig.2. Forsterygion varium. No's 2 \& $2 a: 27$ mm prejuvenile. 


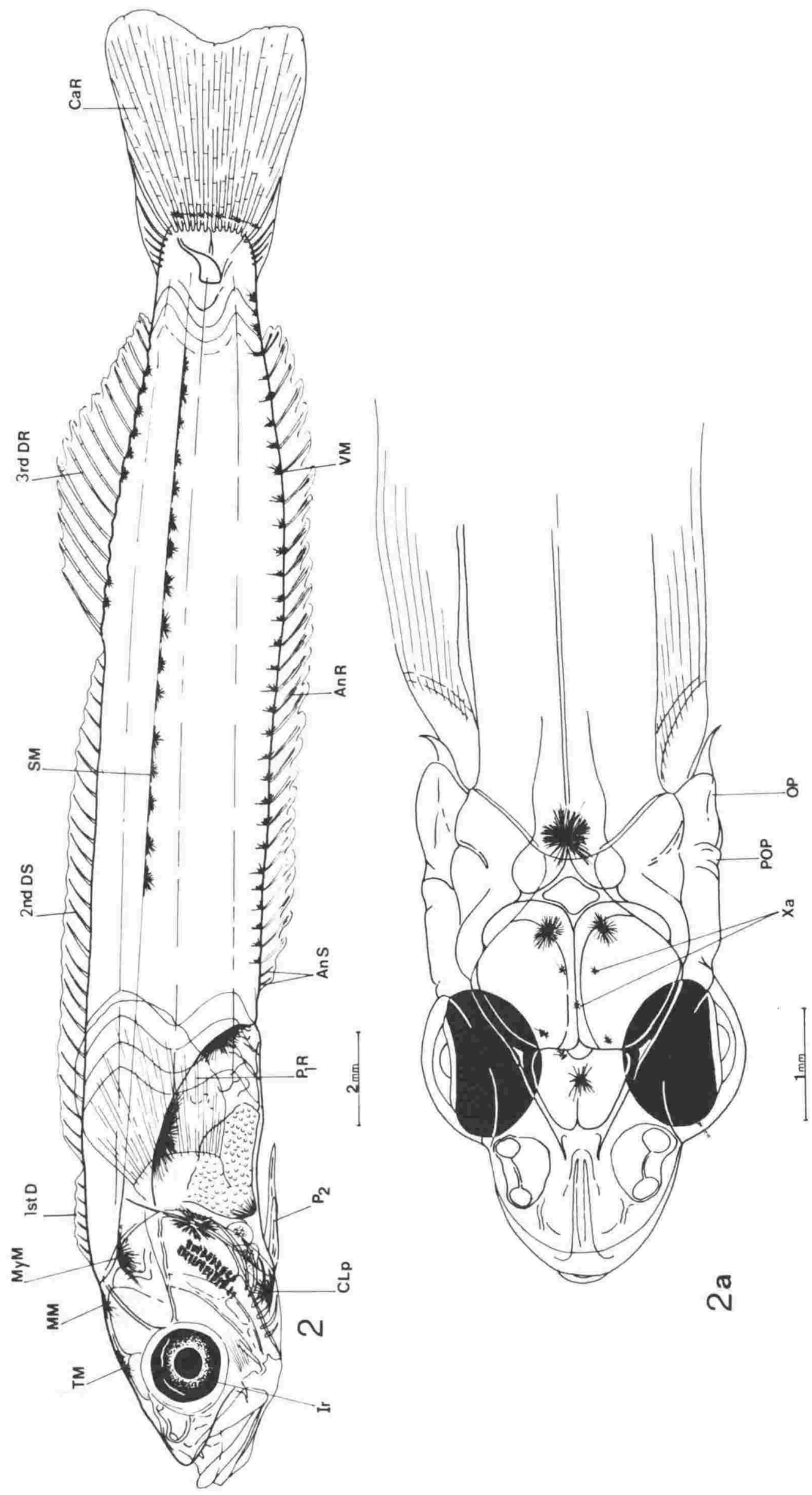

vi
$\frac{1}{4}$ 
262.

Fig.3. Forsterygion varium. Lateral aspect of $28.70 \mathrm{~mm}$ prejuvenile.

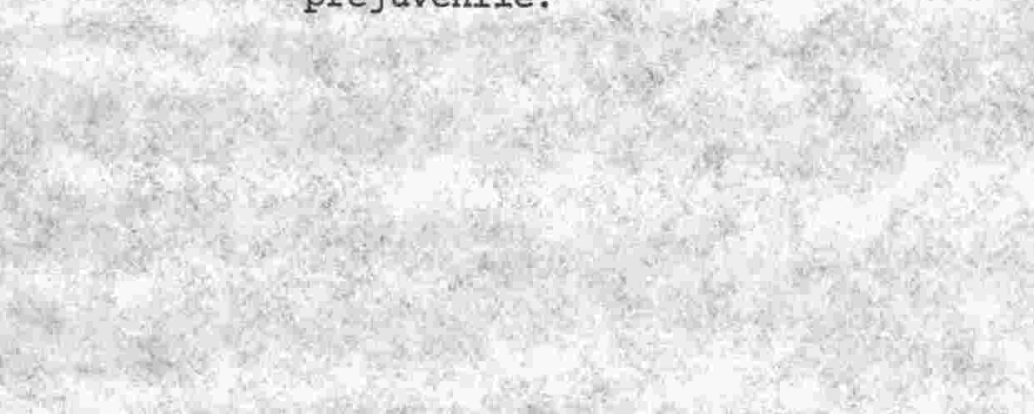

arise 


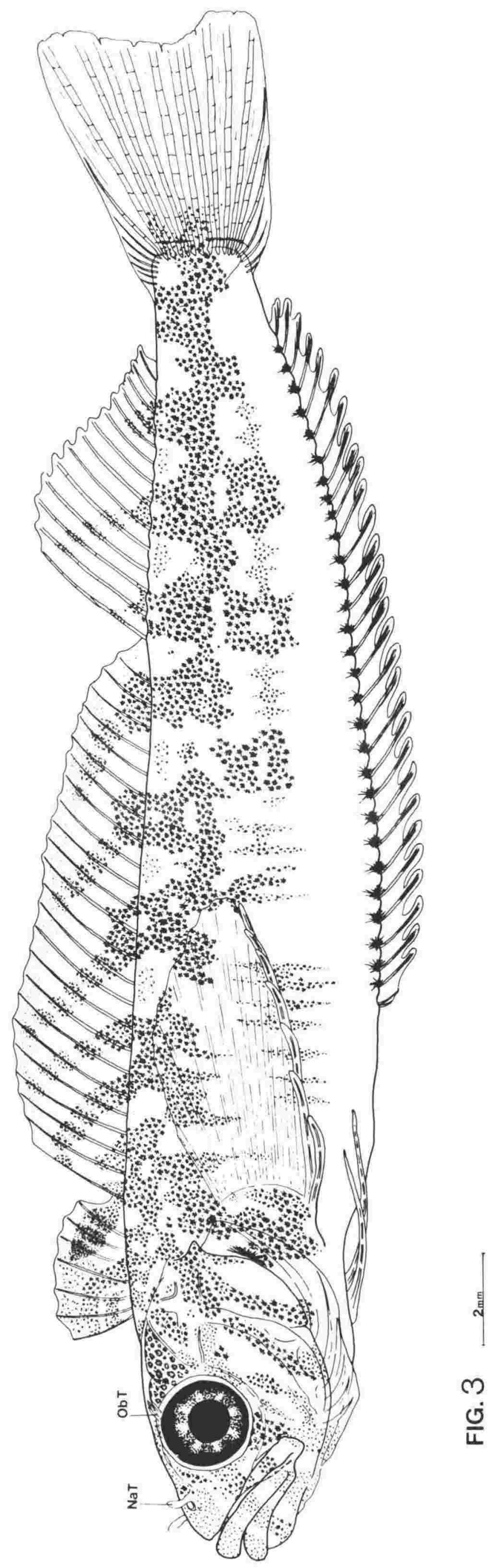


263.

Fig.4. Forsterygion varium. Dorsal aspect of head and trunk of $28.7 \mathrm{~mm}$ prejuvenile. 


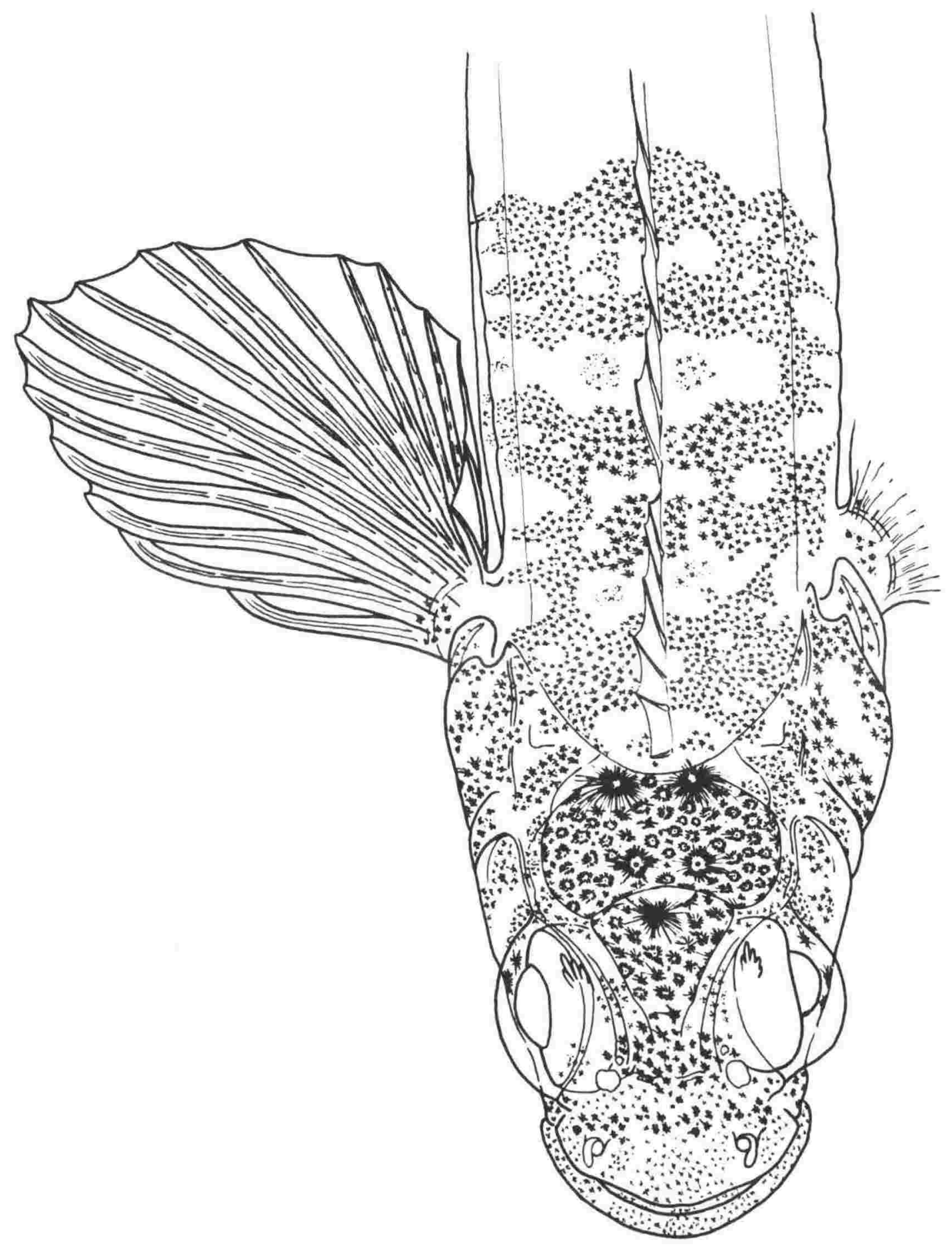


264.

Fig.5. Forsterygion nigripenne. No's 1 \& la: $9.8 \mathrm{~mm}$ larva; 2: $16.5 \mathrm{~mm}$ larva; 3: $26 \mathrm{~mm}$ prejuvenile; 4: $27.5 \mathrm{~mm}$ prejuvenile. 


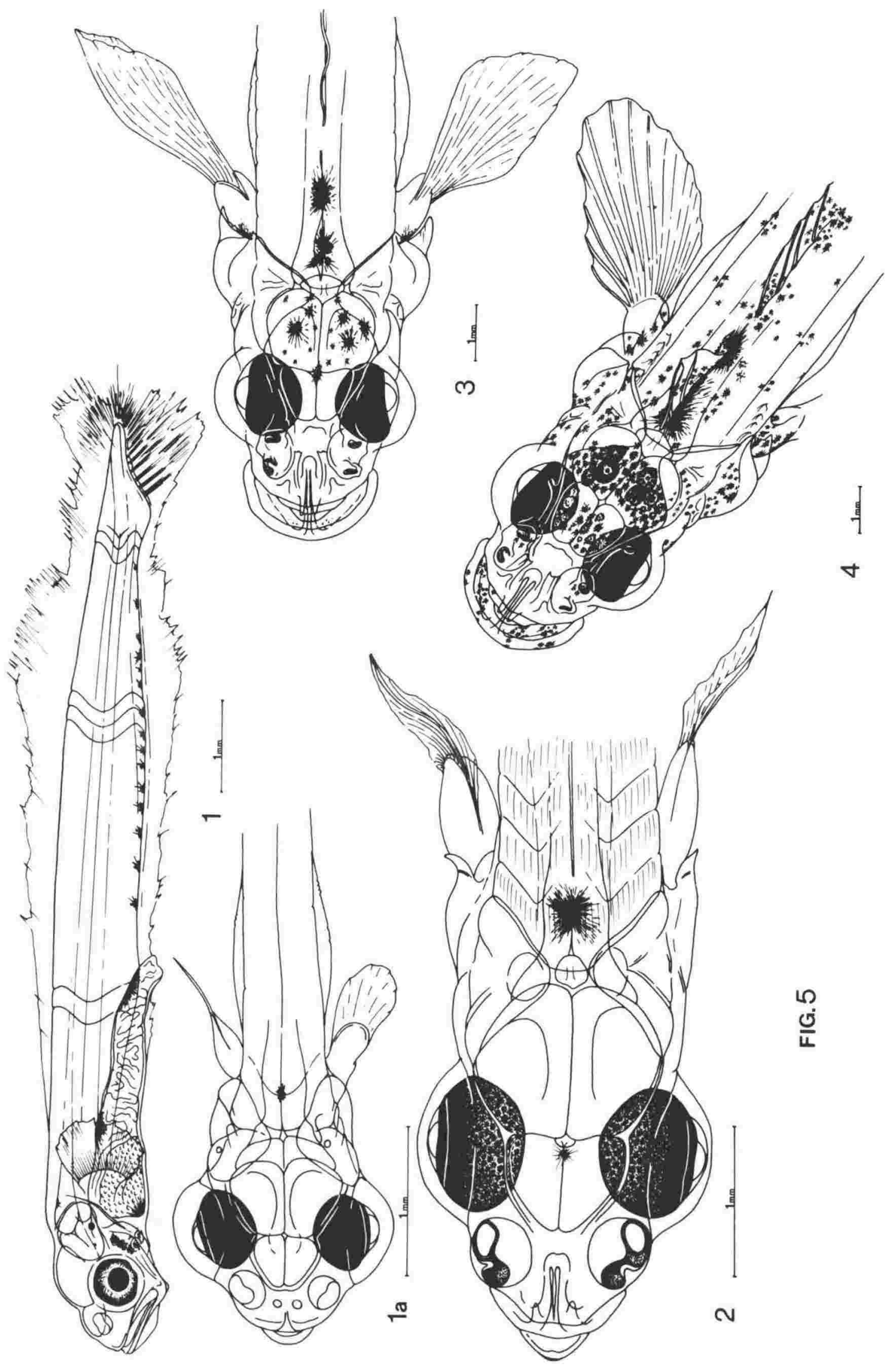


265.

Fig.6. Forsterygion nigripenne. No.1: $16.5 \mathrm{~mm}$ larva;

2 \& 2a: $26 \mathrm{~mm}$ prejuvenile; $3: 27.5 \mathrm{~mm}$ prejuvenile. 

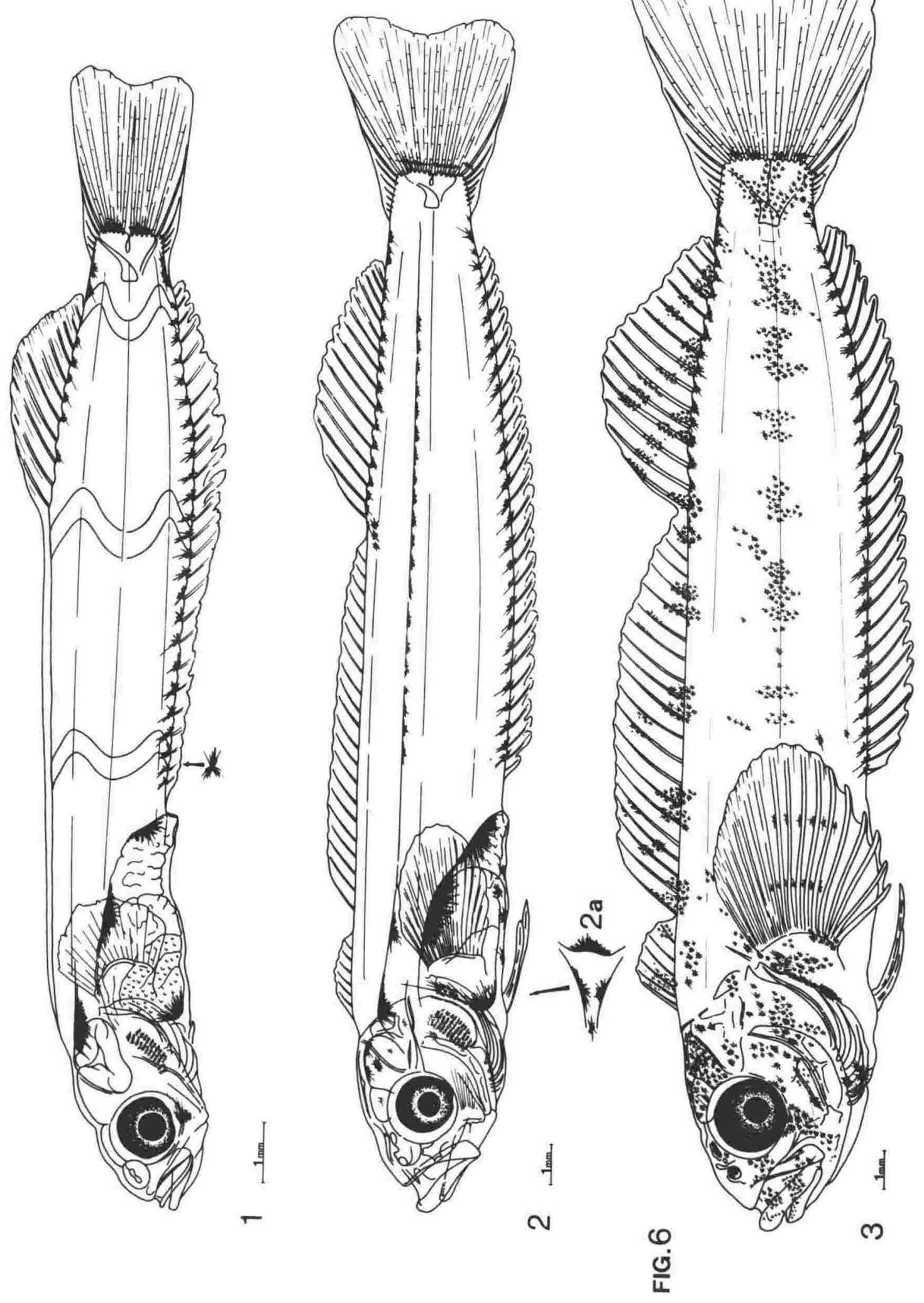
266.

Fig.7. Gilloblennius tripennis, No's 1 , la \& $1 \mathrm{~b}: 7.7 \mathrm{~mm}$ larva; $2 \& 2 a: 9.3 \mathrm{~mm}$ larva; $3 \& 3 a: 14.8 \mathrm{~mm}$ prejuvenile. 


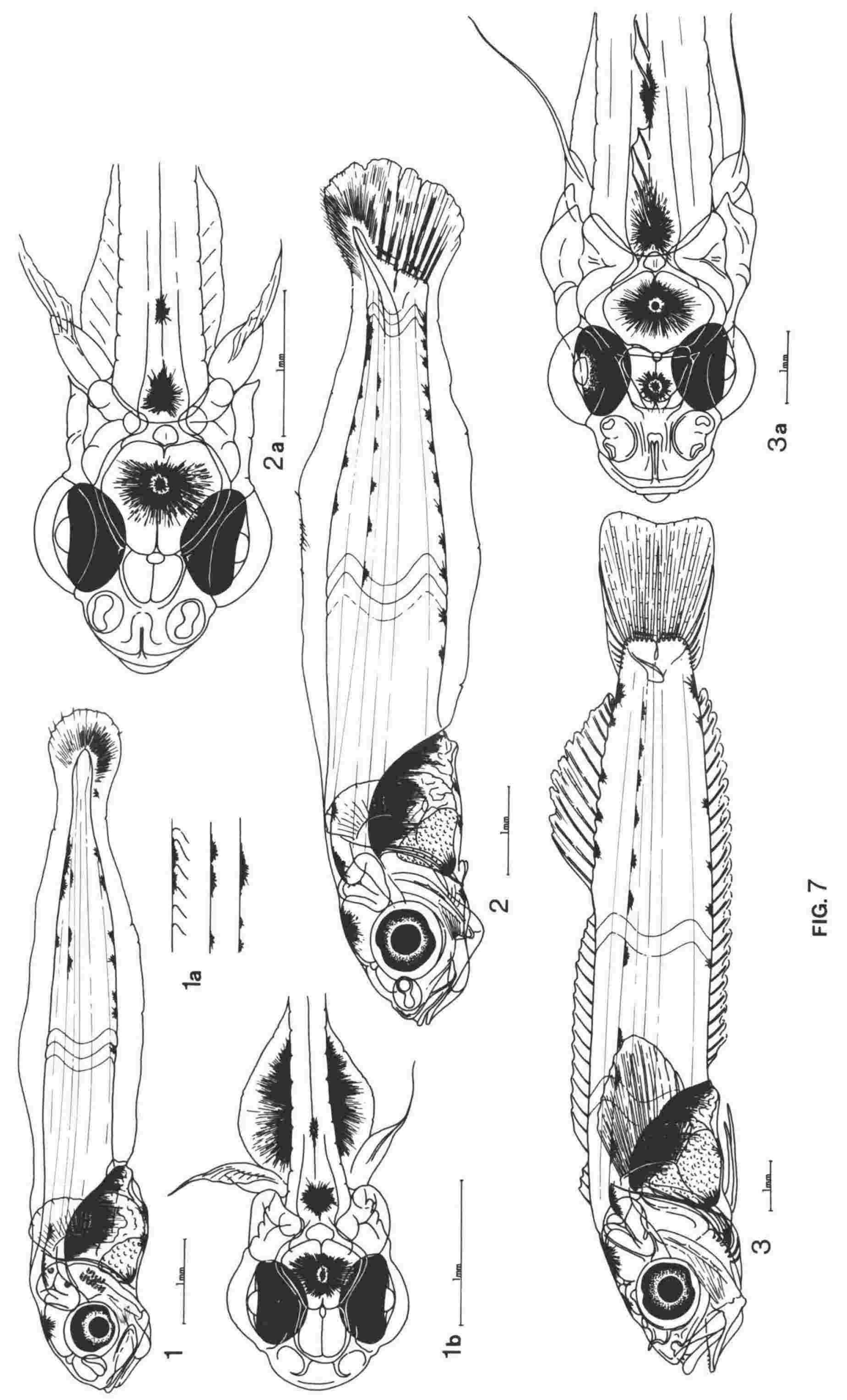


Fig.8. Gilloblennius decemdigitatus. No's 1 \& $1 \mathrm{a}: 9.7 \mathrm{~mm}$ larva; 2, $2 \mathrm{a} \& 2 \mathrm{~b}: 11.4 \mathrm{~mm}$ larva; $3 ; 13.5 \mathrm{~mm}$ larva. 


Fig. 9. Gilloblennius decemdigitatus. No's $1 \& 1 \mathrm{a}$ : lateral and dorsal aspect of $25.7 \mathrm{~mm}$ prejuvenile. 


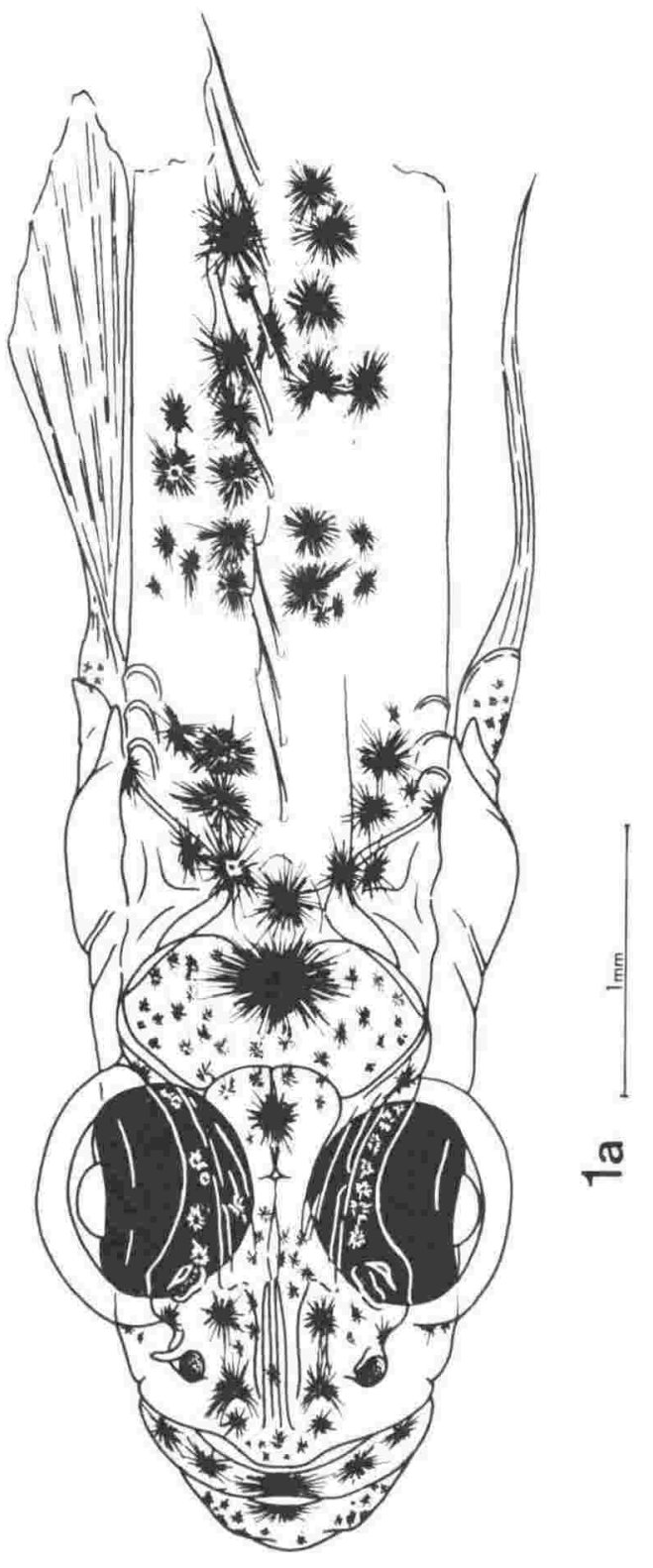

の
$\frac{0}{11}$

\section{t)}



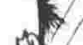
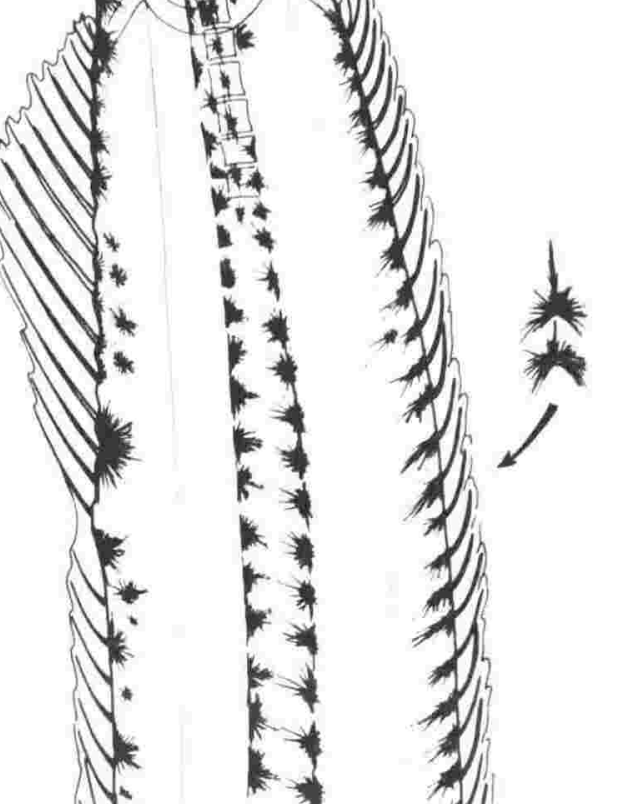

Niv



$+1$




Fig.10. Helcogramma sp.1. No's 1. \& la: $15.8 \mathrm{~mm}$ larva; 2: $20.2 \mathrm{~mm}$ prejuvenile; $3: 21.8 \mathrm{~mm}$ prejuvenile. 


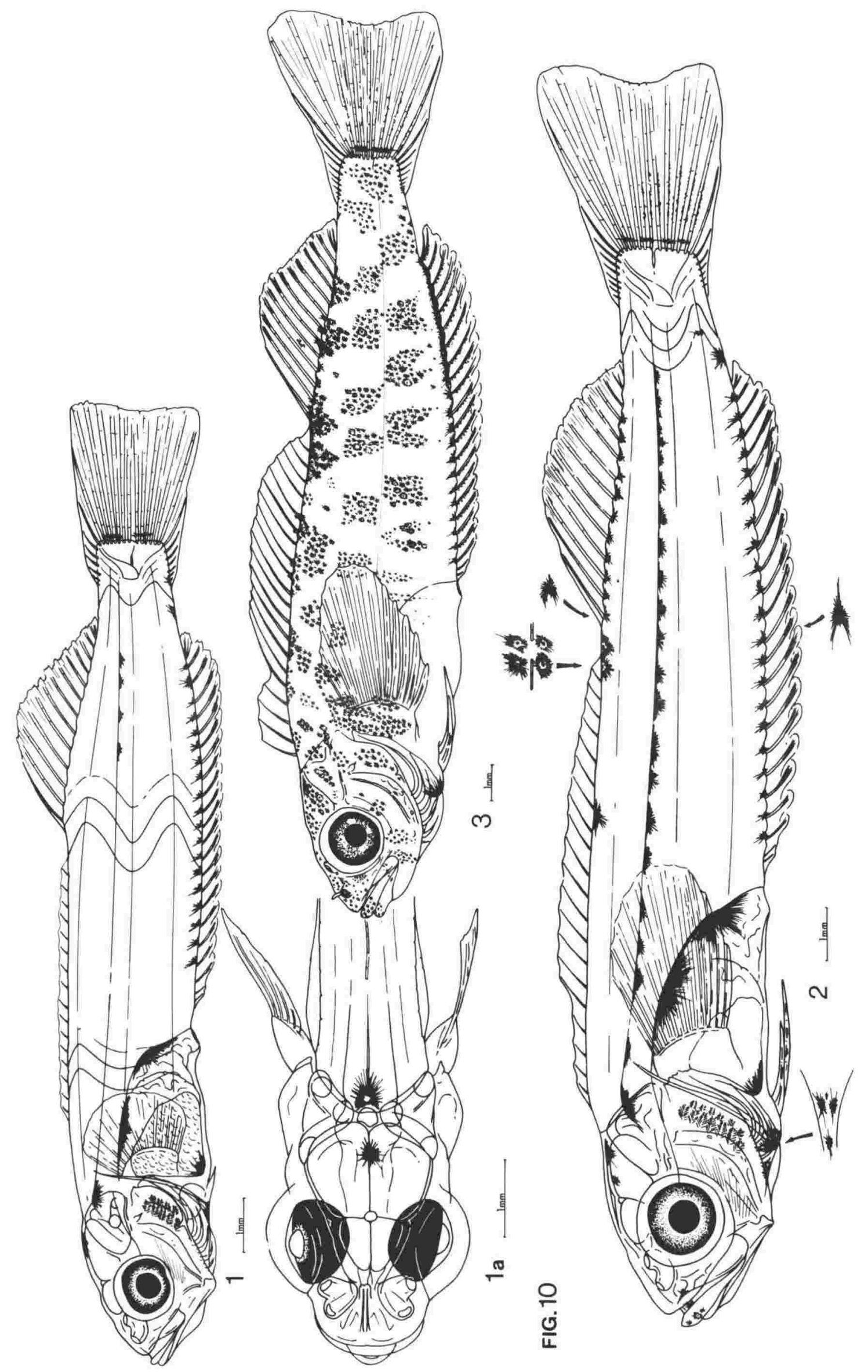


270.

Fig.11. Helcogramma sp.2. No's $1,1 \mathrm{a} \& 1 \mathrm{~b}: 18.0 \mathrm{~mm}$ prejuvenile; $2 \& 2 a:$ Helcogramma sp. 1, $21 \mathrm{~mm}$ juvenile. 





271.

Fig.12. Helcogramma sp.2. No's 1, la \& 1b: $11.2 \mathrm{~mm}$ larva; 2, $2 \mathrm{a} \& 2 \mathrm{~b}: 14.8 \mathrm{~mm}$ larva; $3 \& 3 \mathrm{a}: 16.9 \mathrm{~mm}$ prejuvenile. 

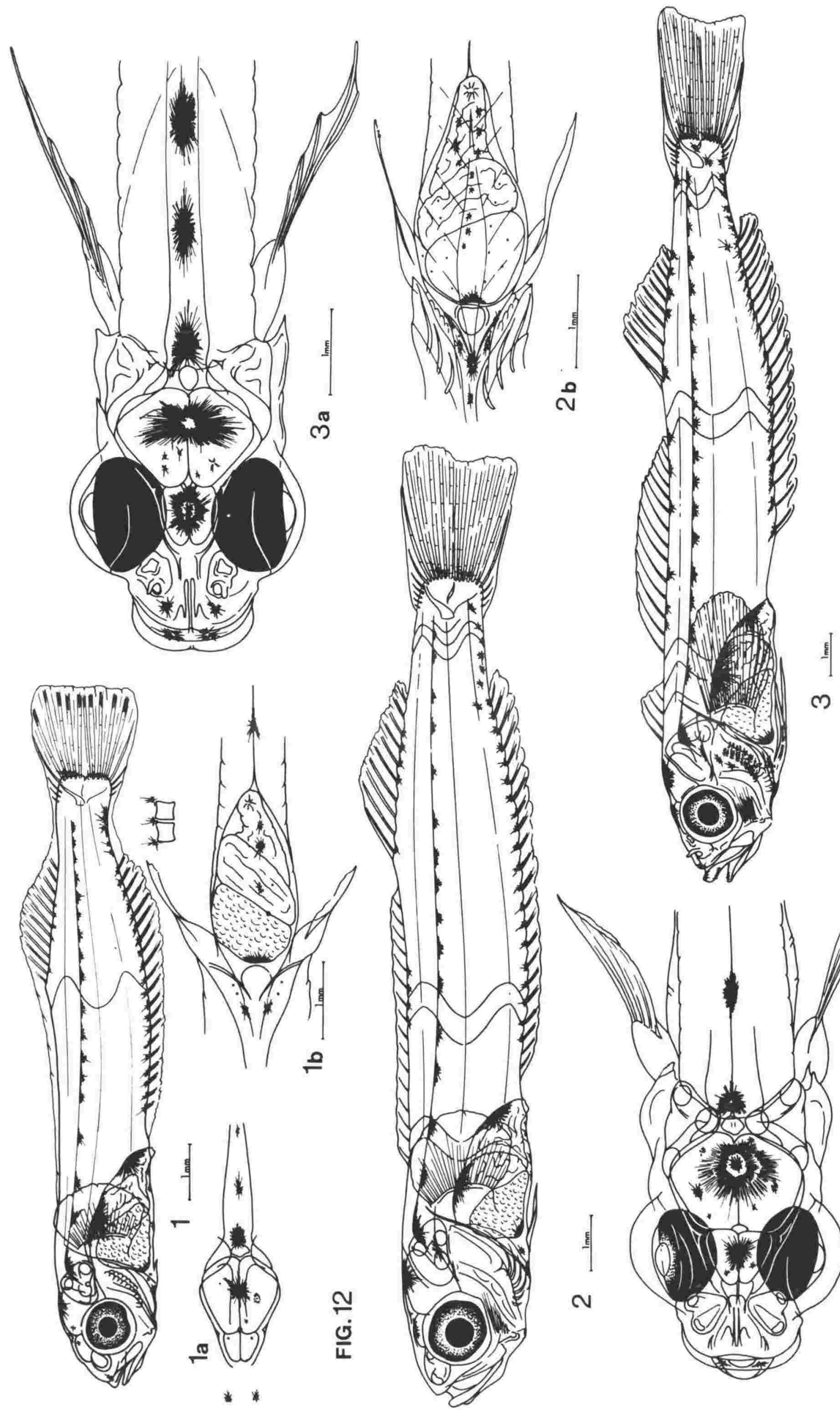

NN.

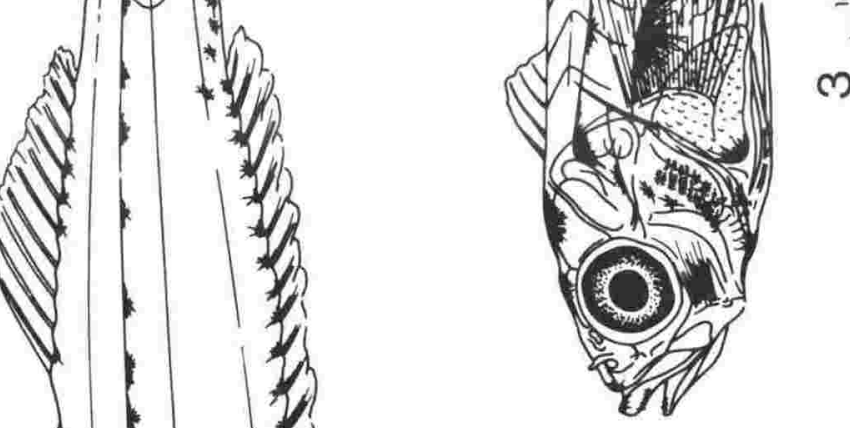


272.

Fig.13. Helcogramma medium. No's $1,1 \mathrm{a} \& 1 \mathrm{~b}: 11.2 \mathrm{~mm}$ larva; 2 , 2a \& 2b: $16.5 \mathrm{~mm}$ prejuvenile; $3 \& 3 \mathrm{a}: 18 \mathrm{~mm}$ prejuvenile. 


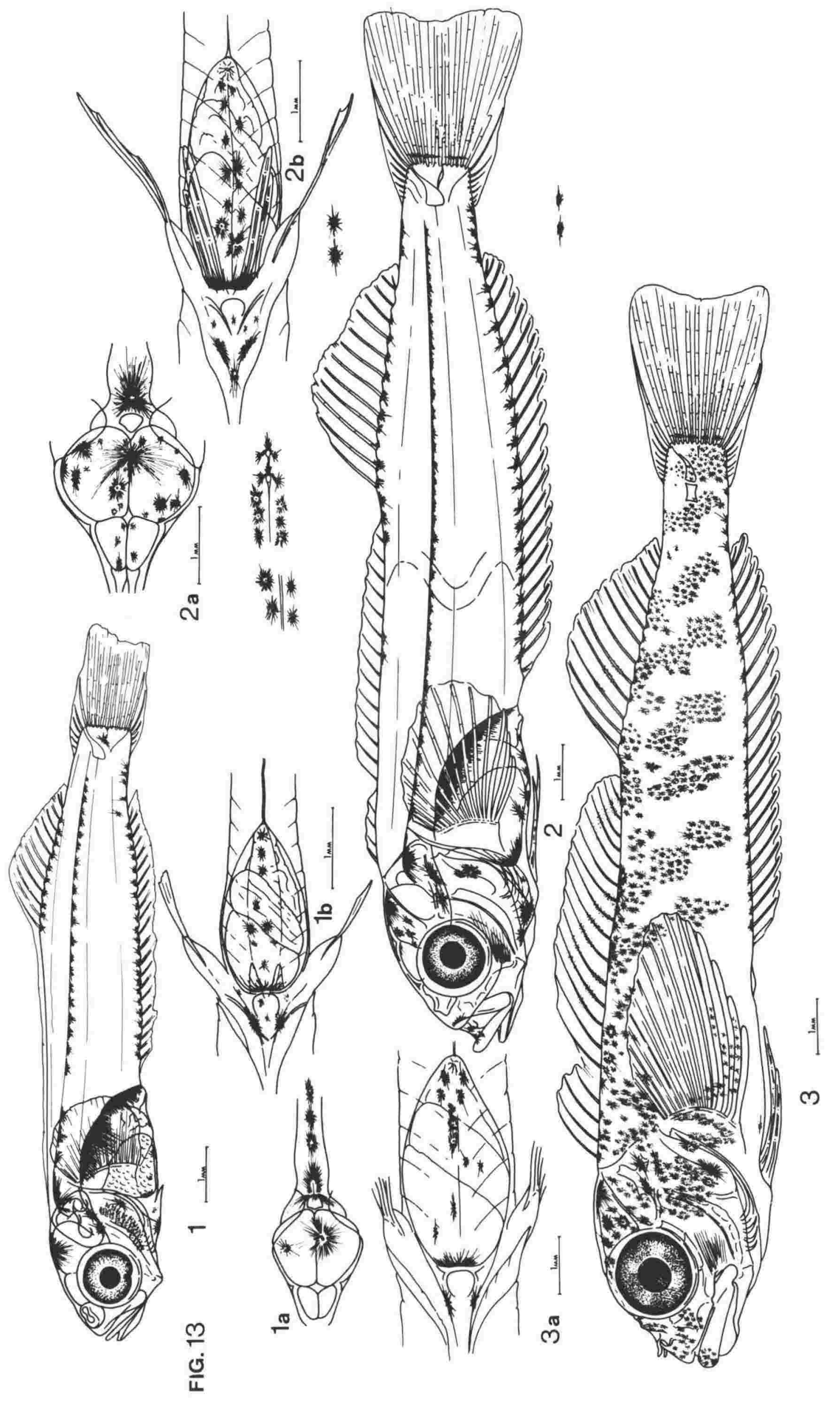


Fig.14. New tripterygiid species. No's 1 \& la: $8.0 \mathrm{~mm}$ larva; 2, $2 \mathrm{a}$ \& $2 \mathrm{~b}: 11.5 \mathrm{~mm}$ larva; 3 \& $3 \mathrm{a}: 14.6 \mathrm{~mm}$ prejuvenile. 


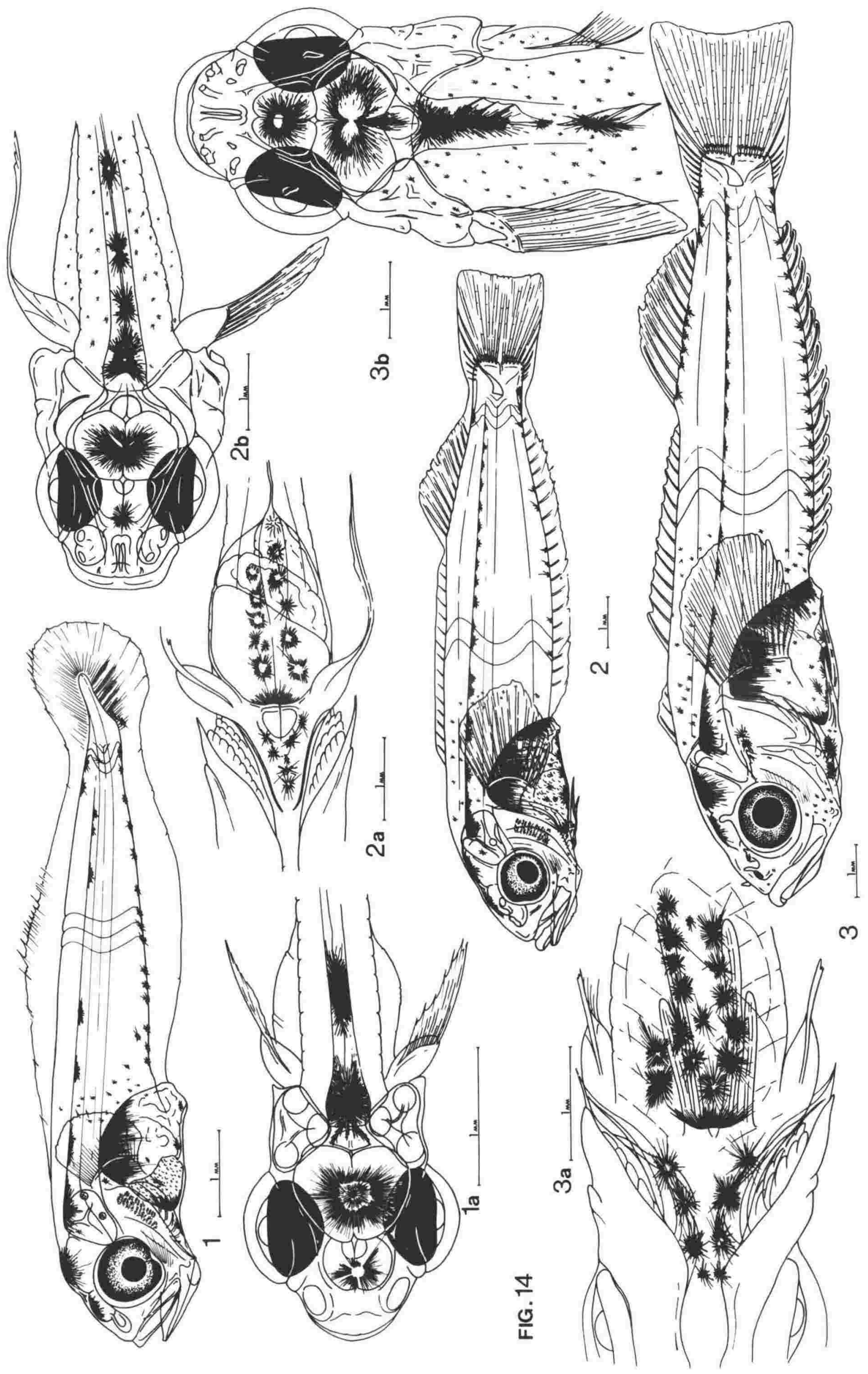


274.

Fig.15, Notoclinus compressus. No's 1 , la \& $1 \mathrm{~b}: 6.4 \mathrm{~mm}$ larva; $2 \& 2 a: 8.5 \mathrm{~mm}$ iarva; $3 \& 3 a: 11.7 \mathrm{~mm}$ prejuvenile; 4: $12.8 \mathrm{~mm}$ prejuvenile. 

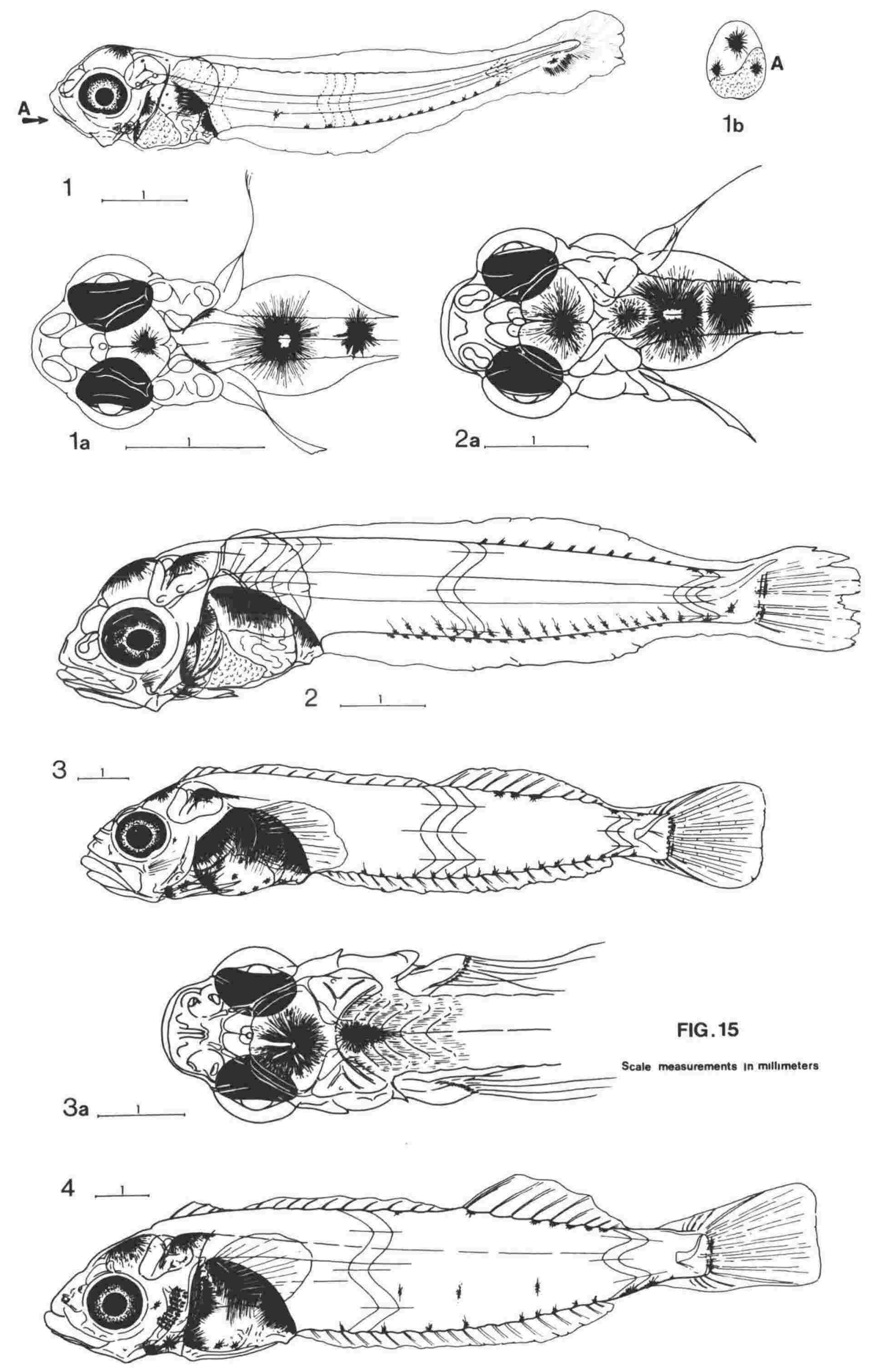
Fig.16. Notoclinus compressus. No.1: $12.8 \mathrm{~mm}$ prejuvenile; $2,2 \mathrm{a}, 2 \mathrm{~b} \& 2 \mathrm{c}: 13.5 \mathrm{~mm}$ prejuvenile; 3 : arrangement of melanic pigment about the gut; $4: 14.4 \mathrm{~mm}$ juvenile. 

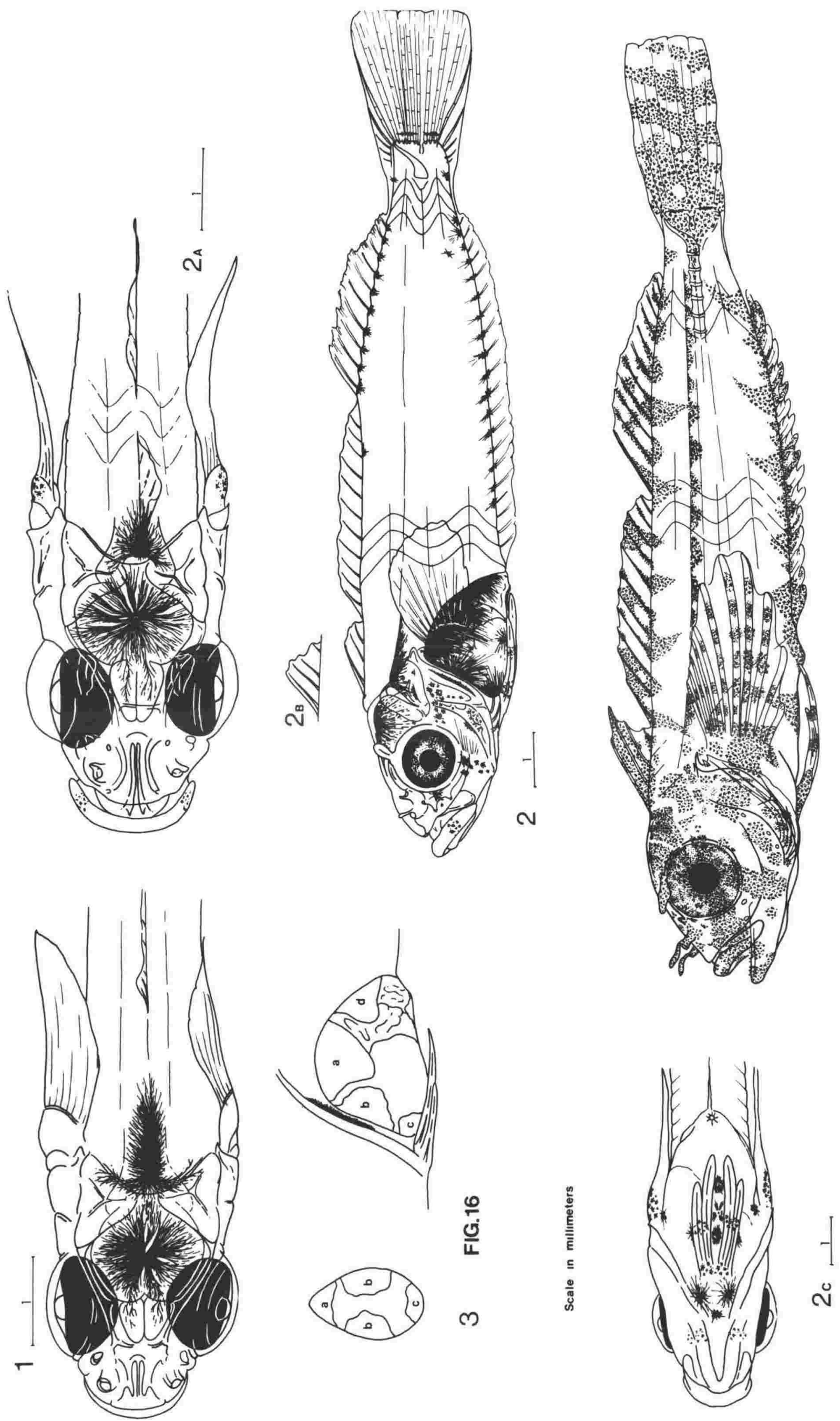
276.

Fig.17. Notoclinus fenestratus. No's 1 \& la: $9.8 \mathrm{~mm}$ larva; 2: Ericentrus rubrus, $10.5 \mathrm{~mm}$ larva; $3 \& 3 \mathrm{a}: 15.9 \mathrm{~mm}$ prejuvenile. 

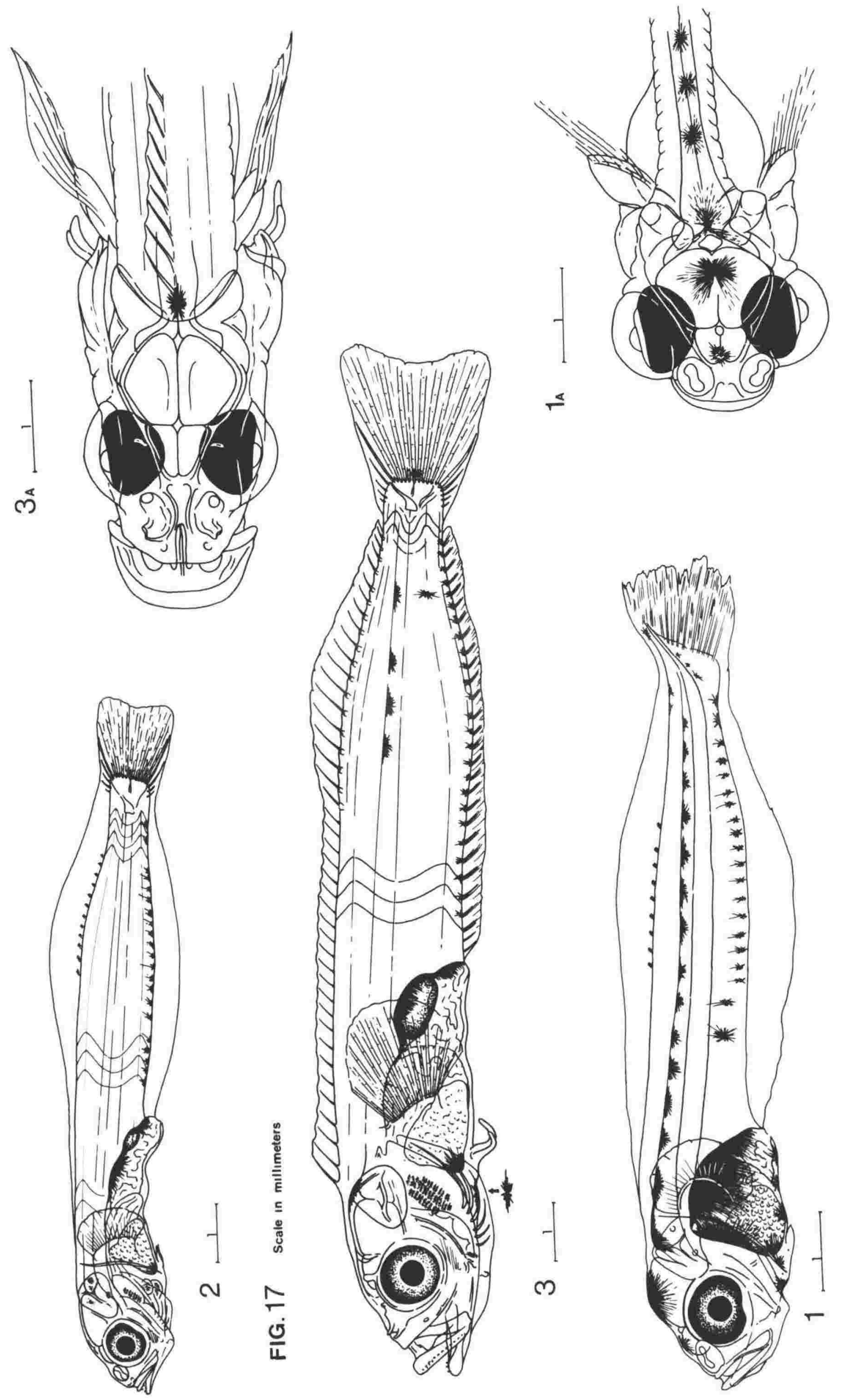
277.

Fig.18. Cologrammus flavescens. No's 1 \& la: $10 \mathrm{~mm}$ larva; 2 \& 2a: $15.1 \mathrm{~mm}$ larva; 3 \& $3 a: 19.3 \mathrm{~mm}$ prejuvenile. 


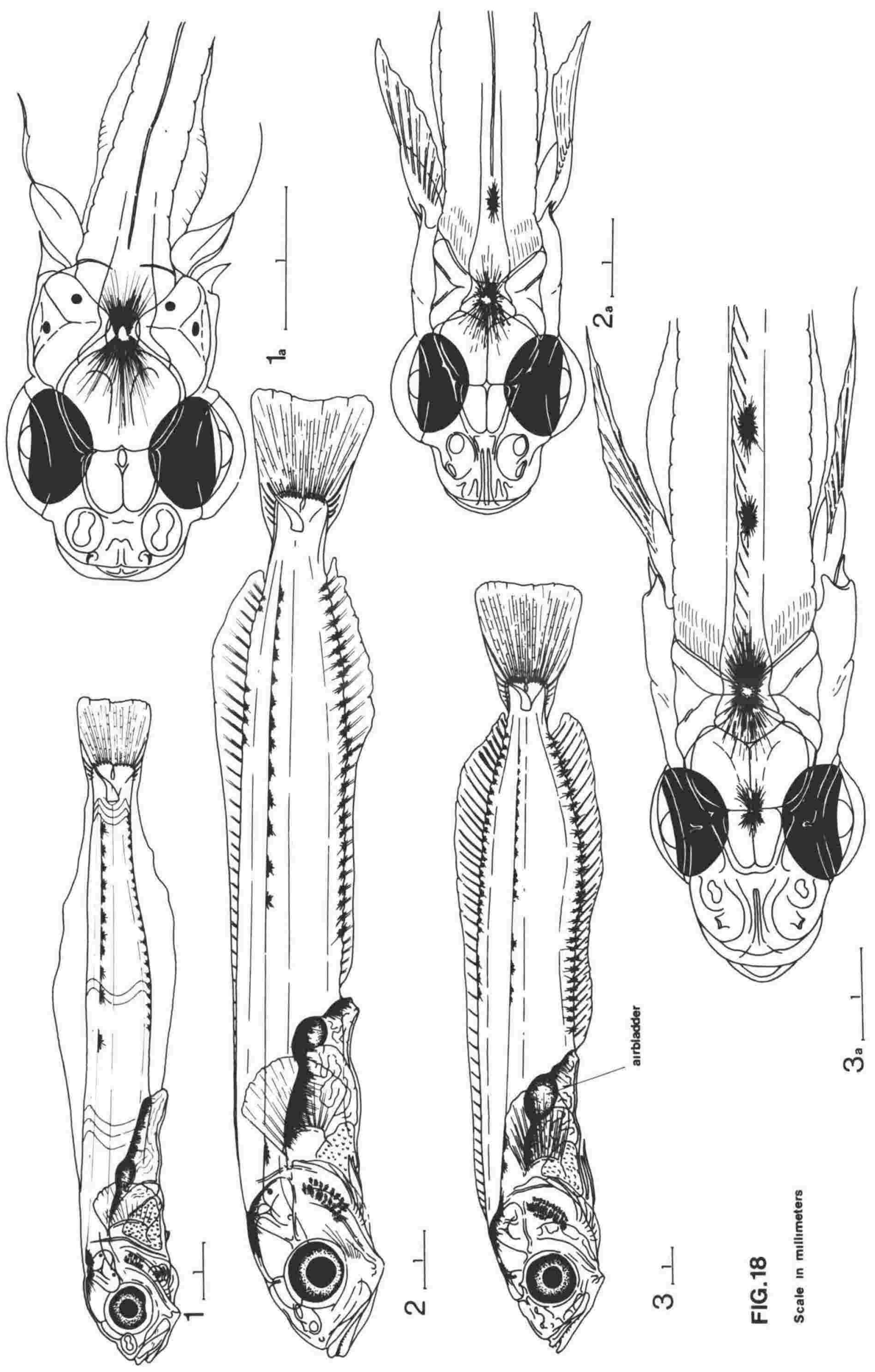


Fig.19. Trachelochismus melobesia. No's 1 \& la: prolarva, $4.8-5.5 \mathrm{~mm} ; 2: 6.3 \mathrm{~mm}$ larva; $3: 7.0 \mathrm{~mm}$ larva; 4 \& $4 a ; 7.85 \mathrm{~mm}$ prejuvenile; $5: 8.7 \mathrm{~mm}$ juvenile. 

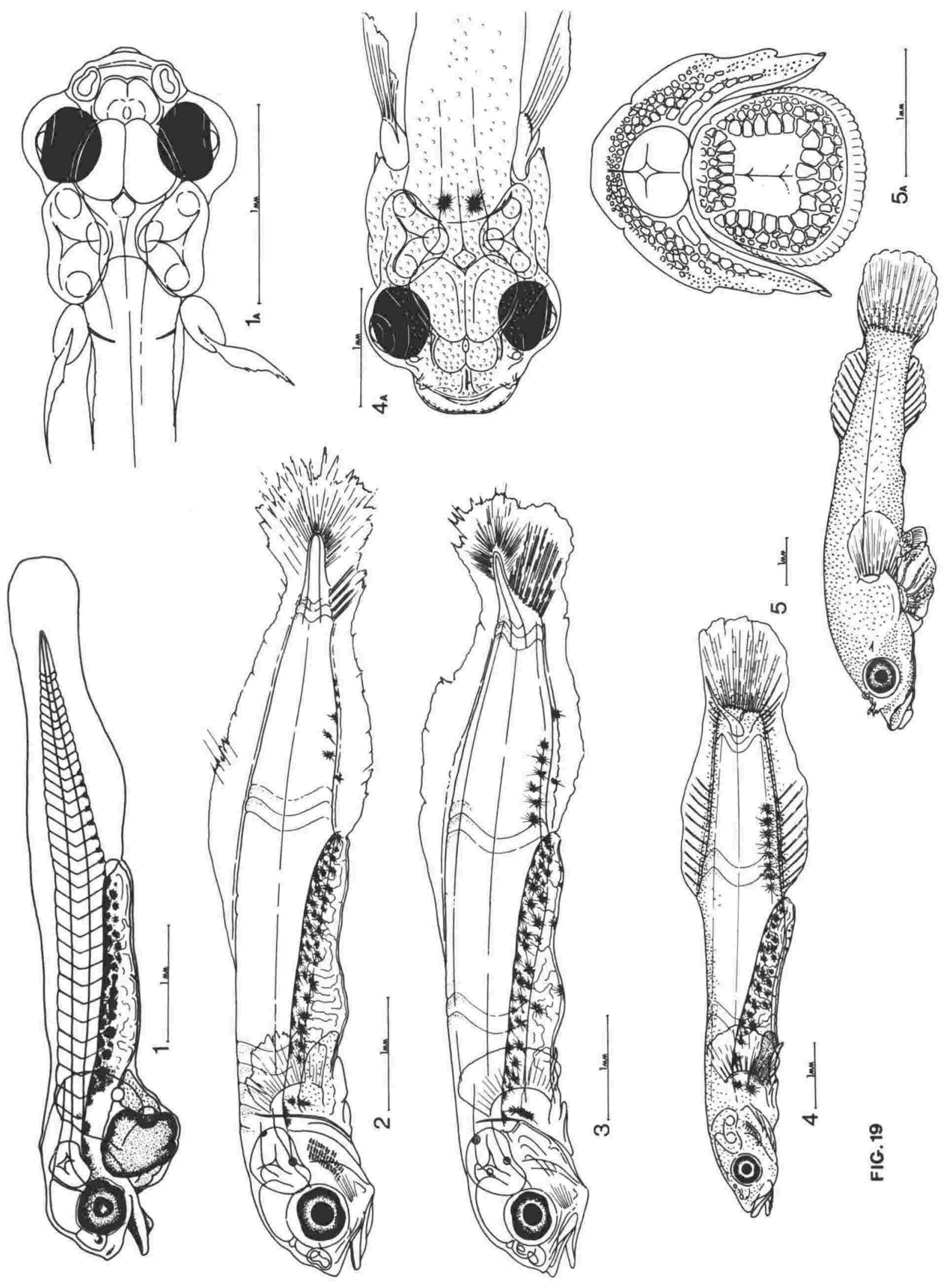
Fig.20. Diplocrepis puniceus. No's 1 \& 2: prolarva, 5.00-6.05 mm; $3 \& 4: 6.2 \mathrm{~mm}$ larva; $5 \& 6: 7.7 \mathrm{~mm}$ larva. 

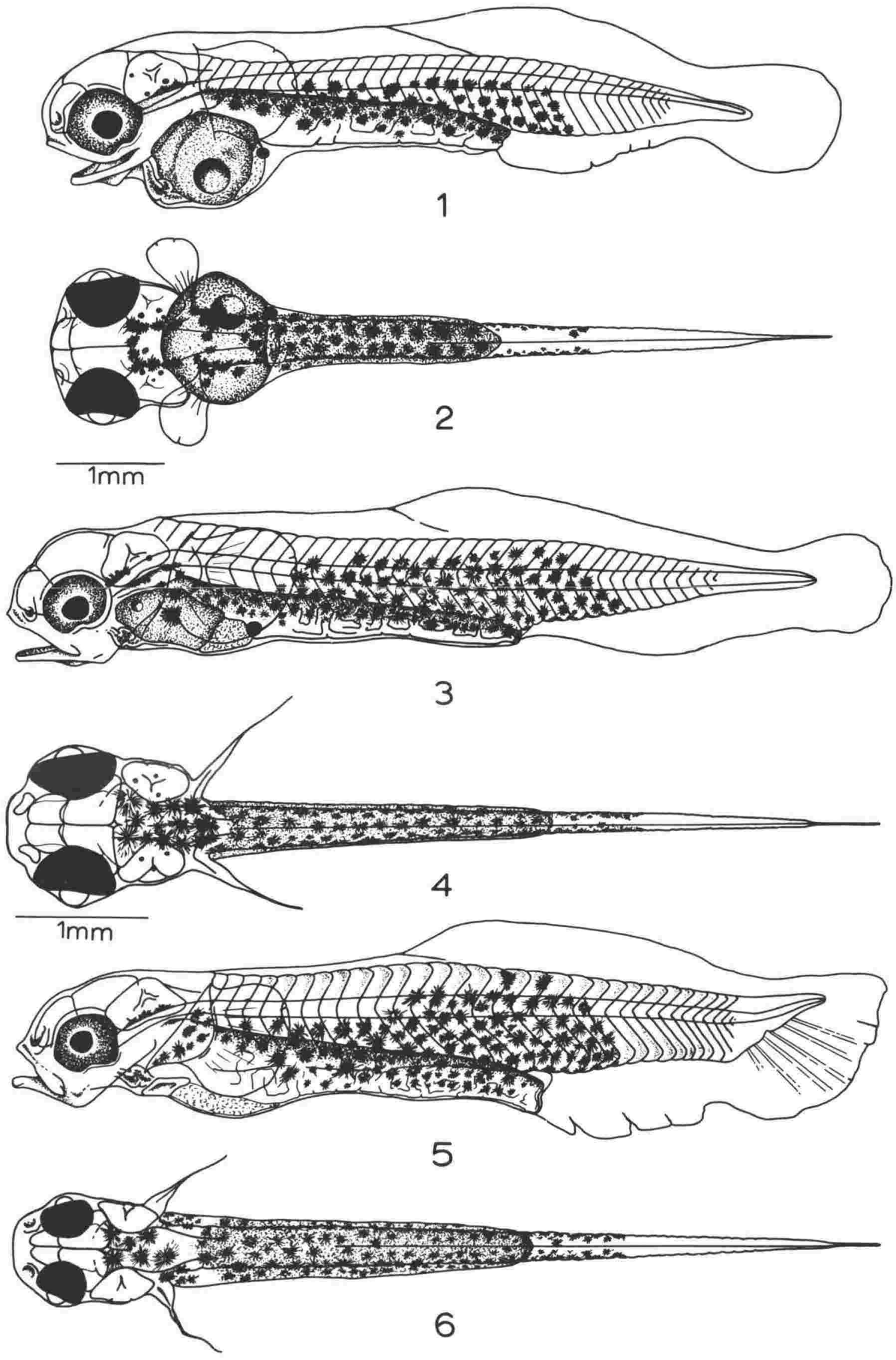

$x^{2} x^{2}+1$

$1 \mathrm{~mm}$ 
280.

Fig.20a. Trachelochismus pinnulatus. No's 1, la \& $1 \mathrm{~b}: 9.6 \mathrm{~mm}$ prejuvenile; $2,2 \mathrm{a} \& 2 \mathrm{~b}$ : Diplocrepis puniceus, $9.2 \mathrm{~mm}$ larva; $3 \& 3 a: 9.7 \mathrm{~mm}$ prejuvenile. 


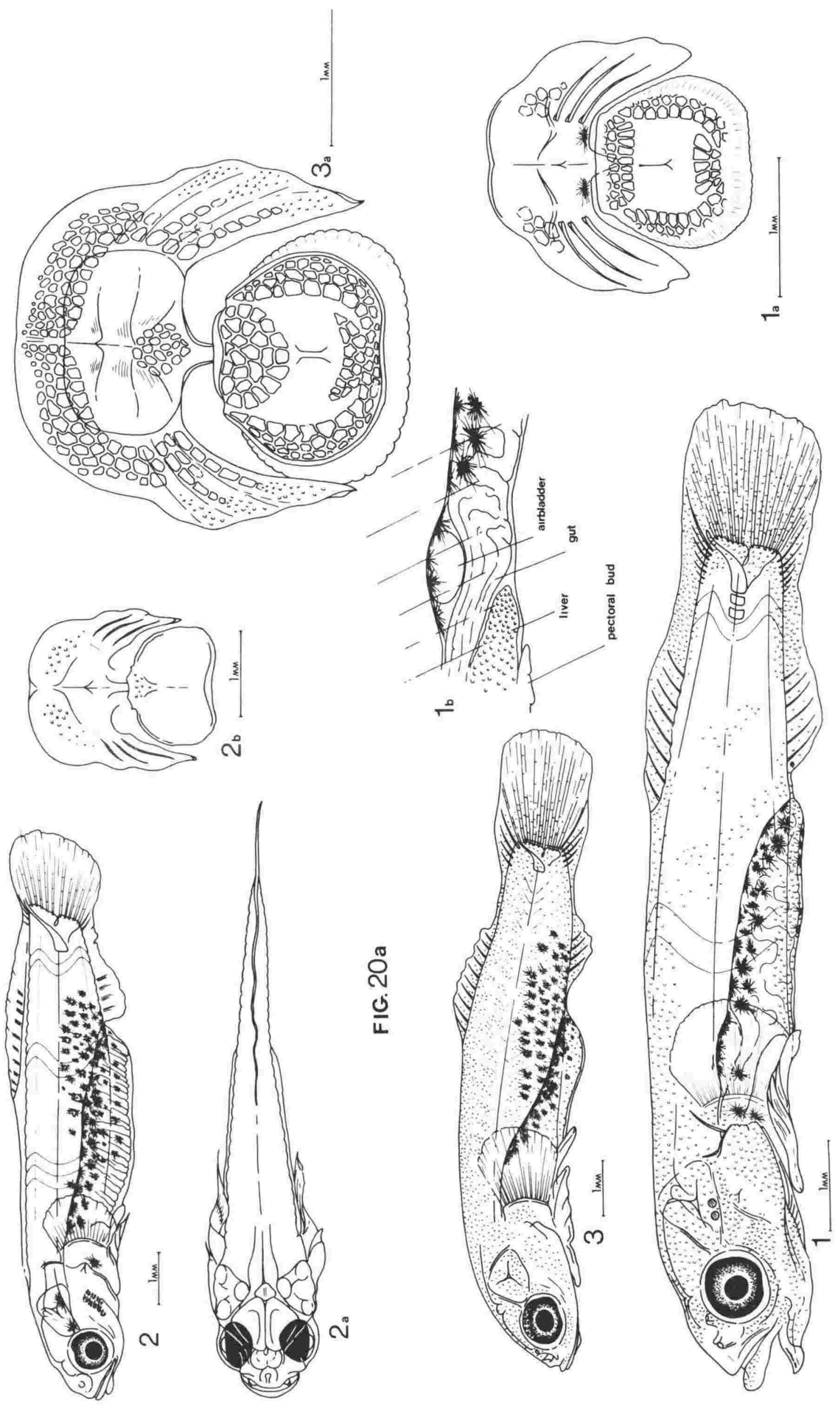


281.

Fig.21. Gastroscyphus hectoris. No's 1 \& $1 \mathrm{~b}: 5.4 \mathrm{~mm}$ larva;

$2 \& 2 a: 10.6 \mathrm{~mm}$ prejuvenile; $3,3 \mathrm{a} \& 3 \mathrm{~b}: 9.7 \mathrm{~mm}$ juvenile. 


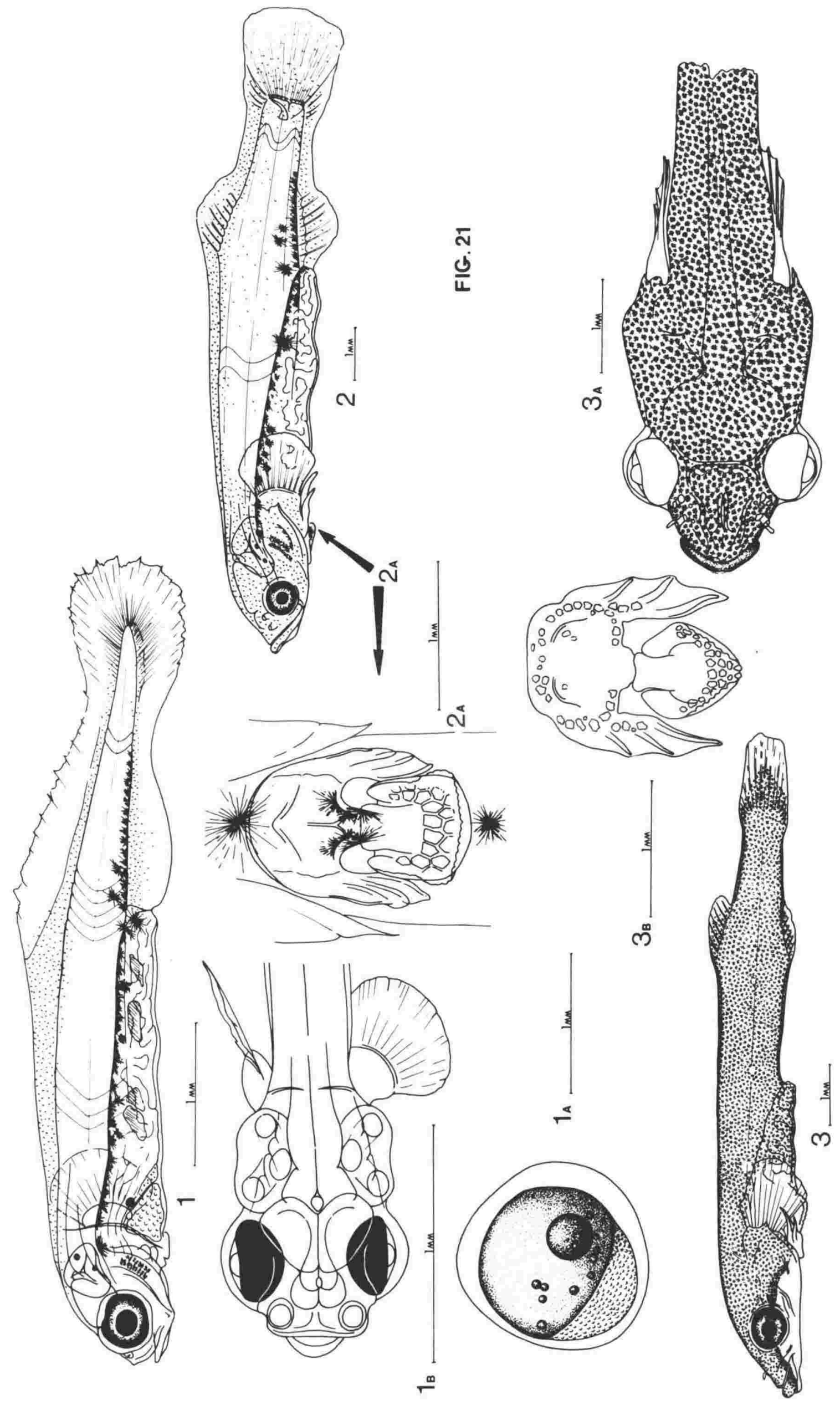


282.

Fig.22. Gastrocyathus gracilis. No's $1 \& 1 \mathrm{a}: 5.5 \mathrm{~mm}$ larva; $2 \& 2 a: 6.9 \mathrm{~mm}$ larva; $3,3 a \& 3 b: 8.2 \mathrm{~mm}$ prejuvenile. 

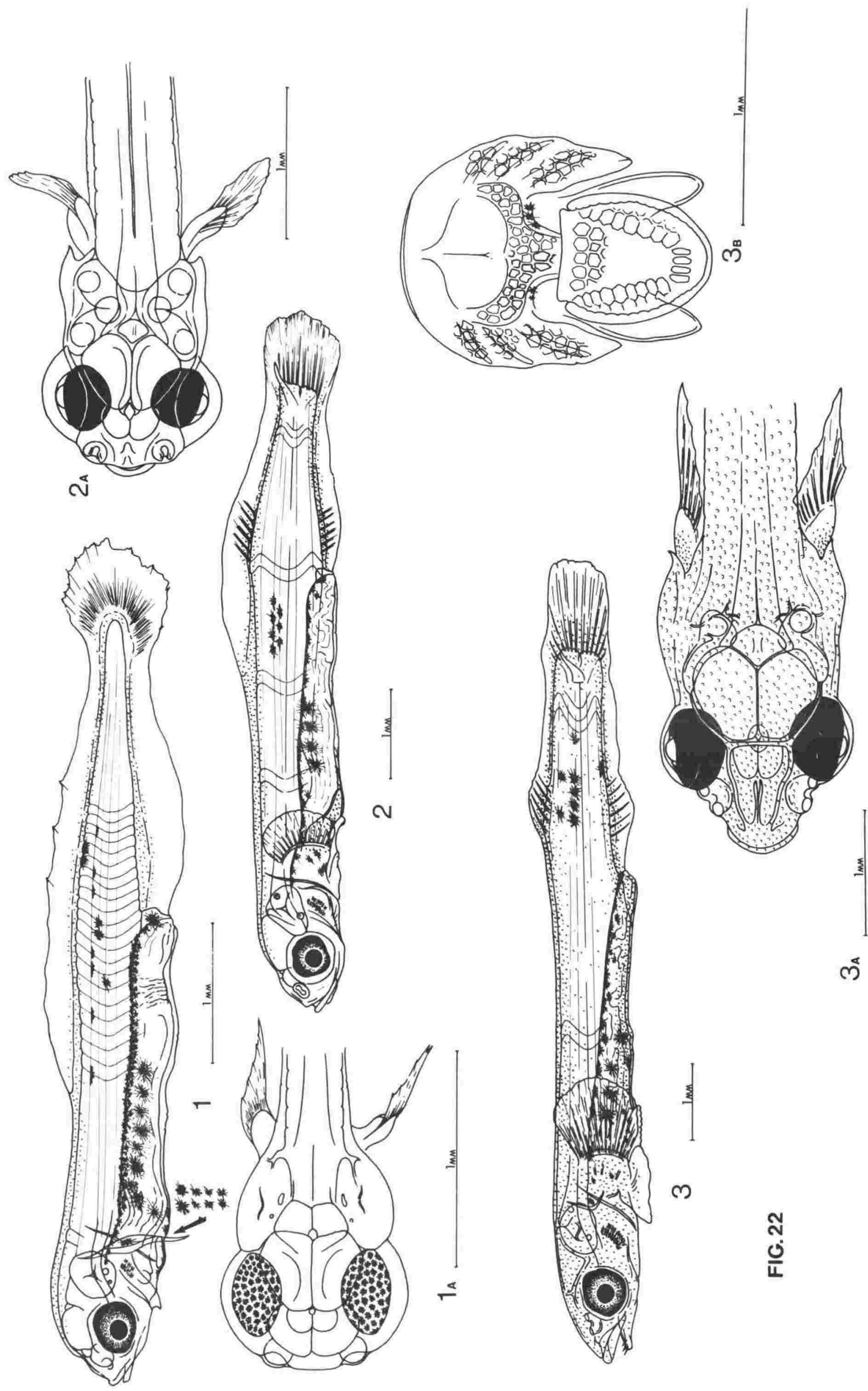


\section{Literature Cited}

Aboussouan, A. 1975.

Ichthyoplankton. Report of the CICAR ichthyoplankton workshop Carangid taxonomy. Unesco technical papers in marine science, (20) : 20-22.

Adron, J. W., Blair, A. and Cowey, C. B. 1974.

Rearing of plaice (Pleuronectes platessa) larvae to metamorphosis using an artificial diet. Fishery Bull. Fish Wildl. Serv. U.S., $72(2): 353-357,2$ fig., 1 tab.

Ahlstrom, E. H. 1975.

Ichthyoplankton. Report of the CICAR ichthyoplankton workshop Taxonomy introductory session, and Myctophid, Gonostomatid

and Sternoptychid taxonomy. Unesco technical papers in marine science, (20) $: 14-15,18-19$.

Ahlstrom, E. H. and Ball, O. P. 1954.

Description of eggs and larvae of jack mackerel (Trachurus

symmetricus) and distribution and abundance of larvae in 1950 and

1951. Fishery Bull. Fish Wildl. Serv. U.S., 56: 209-245, 28 fig.

Ahlstxom, E. H. and Counts, R. C. 1958.

Development and distribution of Vinciguerria lucetia and related species in the Eastern Pacific. Fishery Bull. Fish Wildl. Serv. U.S., $58(139): 359-416,29$ fig., 44 tab.

Anderton, T. 1906.

Observations on New zealand fishes, etc., made at the Portobello Marine Fish-hatchery. Trans. Proc. N.Z. Inst., 39:477-96.

Applegate, R. I. 1966.

Pyloric caeca counts as a method for separating the advanced fry and fingerlings of largemouth and spotted basses. Jrans. Am. Eish. Soc. , $95: 226$.

Aprieto, V. L. 1974.

Early development of five carangid fishes of the Gulf of Mexico and the South Atlantic Coast of the United States. Fishery BuIl. Fish Wildl. Serv. U.S., $72(2): 415-443,8$ fig., 12 tab.

Atz, J. W. 1964.

A working bibliography on rearing larval marine fishes in the laboratory. Fishery Bull. Fish Wildl. Serv. U.S., Rep. 63:95-102. 
Baker, A. N. 1971.

Reproduction, early life history, and age-growth relationships of the New Zealand pilchard, Sardinops neopilchardus (Steindachner). Published Ph.D. thesis, Victoria University of Wellington, New Zealand.

Baker, A. N. 1972.

Reproduction, early life history, and age-growth relationships of the New Zealand pilchard, Sardinops neopilchardus (Steindachner). Fish. Res. Bull. N.z., (5) : 64 pp., 39 fig., 9 tab.

Baker, A. N. 1973.

Spawning and development of the New Zealand sprat, Sprattus antipodum (Hector). Zool. Publs Vict. Univ. Wellington, (62): $12 \mathrm{pp}, 18 \mathrm{fig}$. Balinsky, B. I. 1948.

On the development of specific characters in cyprinid fishes. Proc. zool. Soc. Lond., 118 (2):335-344, 12 fig., 1 tab.

Balinsky, B. I. 1965.

An introduction to embryology. Philadelphia and London:

W. B. Saunders Co., 673 pp., 443 fig., 23 tab.

Balon, E. K. 1975.

Terminology of intervals in fish development. J. Fish. Res. Bd Can. $32(9): 1663-1670,4 \mathrm{fig}$.

Barkley, R. A. 1972.

Selectivity of towed-net samplers. Fishery Bull. Fish Wildl. Serv. U.S., 70(3):799-820, 10 fig., 3 tab.

Battle, H. I. 1944 .

The embryology of the Atlantic salmon (Salmo salar Linnaeus). Can. J. Res., 22(5):105-125, 39 fig., 4 tab.

Benzie, V. 1968b.

A consideration of the whitebait stage of Galaxias maculatus attenuatus (Jenyns). N.Z. J1 mar. Freshwat. Res., 2(3):559-573, $4 \mathrm{fig.,} 5$ tab. (Benzie, 1968a is recorded in the bibliography). Benzie, v. 1968c.

Stages in the normal development of Galaxias maculatus attenuatus (Jenyns). N.Z. J1 mar. Freshwat. Res., 2(4):606-627, 13 fig.

Benzie, V. 1968d.

The life history of Galaxias vulgaris Stokell, with a comparison with G. maculatus attenuatus. N.Z. JI mar. Freshwat. Res., 2(4):628-653, 12 fig., 4 tab. 
Berrien, P. L. 1975.

A description of Atlantic mackerel, Scomber scombus, eggs and early larvae. Fishery Bull. Fish Wildl. Serv. U.S., 73(1):186-192,2 fig.,2 tab. Berry, F. H. 1959.

Young Jack Crevalles (Caranx species) off the Southeastern Atlantic coast of the United States. Fishery Bull. Fish Wildl. Serv. U.S., 59(152):417-535, 98 fig., 23 tab.

Berry, F. H. 1964.

Aspects of the development of the upper jaw bones in teleosts.

Copeia, (2):375-384, 10 fig.,

Bertelsen, E. 1951.

The Ceratioid fishes. Ontogeny, taxonomy, distribution and biology. Dana Rep., (39):1-276, 14l. fig., I pl.

Bertmar, G. 1959.

On the ontogeny of the chondral skull in Characidae, with a discussion of the chondrocranial base and the visceral chondrocranium in fishes. Acta. Zool. Stockh., 40:203-364, 85 fig.

Billet, F. S. and Wild, A. E. 1975.

Practical studies of animal development. London: Chapman and Hall, 251 pp., 69 fig., $10 \mathrm{pl}$.

Bishae, H. M. 1961.

The effect of salinity on the survival and distribution of larval

and young fish. J. Cons. perm. int. Explor. Mer., $26(2): 166-179,4$ fig. Bjerring, H. C. 1972 .

The rhinal bone and its evolutionary significance. Zool. Scripta, 1:

193-201, $6 \mathrm{fig}$.

Blaxter, J.H.S. 1962 .

Herring rearing. IV Rearing beyond the yolk-sac stage. Mar. Res., (1): $18 \mathrm{pp}$.

Blaxtex, J.H.S. 1968a.

Rearing herring larvae to metamorphosis and beyond. J. mar. biol. Ass. U.K., 48:17-28, 6 fig., 2 tab.

Blaxter, J.H.S. 1968b.

Light intensity, vision, and feeding in young plaice. J.exp. mar. Biol. Ecol., 2:293-307, 8 fig., 1 tab.

Bloch, M. E. and Schneider, J. G. 1801.

M. C. Blochii... Systema Ichthyologiae iconibus cx illustratum.

Post obitum auctoris opus inchoatum absolvit, correxit, interpolavit 
Jo. Gottlob Schneider. Berlin, $1 x+584$ pp., 110 col.pl. Böhlke, J. 1957.

A review of the blenny genus Chaenopsis, and the description of a related new genus from the Bahamas. Proc. Acad. nat. Sci. Philad., 59:81-103, 3 fig., 5 tab., 2 pl.

Böhlke, J. E. and Chaplin, C. G. 1968.

Fishes of the Bahamas and adjacent tropical waters. Livingstone Publishing Co., Philadelphia: I-XXII, 1-77l, $36 \mathrm{pl}$.

Böhlke, J. E. and Robins, C. H. 1974.

Description of a new genus and species of clinid fish from the Western Caribbean, with comments on the families of the Blennioidea. Proc. Acad. nat. Sci. Philad., $126(1): 8$ pp., 4 fig.

Breder, C. M. 1941.

On the reproductive behaviour of the sponge blenny, Paraclinus marmoratus (Steindachner). Zoologica, N.Y., 26(22):233-236, 6 fig. Breder, C. M. 1962.

On the significance of transparency in Osteichthid fish eggs and larvae. Copeia, (3):561-567.

Breder, C. M. 1969.

Aspects of melanism in acanthopterygian fishes. Zoologica, N.Y., 54:105-133, 5 fig., 5 tab., 1 map, 1 pl.

Briggs, J.C. 1955.

A monograph of the clingfishes (order Xenopterygii). Stanford ichthyol. Bull., 6:224 pp., $114 \mathrm{fig.,} 1$ tab.

Brisout de Barneville, C.N.F. 1846.

Note sur un nouveau genre de la famille des Discoboles. Revue Zoologique par la Societe Cuvierienne Paris, 9:212-214.

Castle, P.H.J. 1968.

The world of eels. Tuatara, 16(2):85-97, $2 \mathrm{fig}$.

Castle, P.H.J. 1969.

An index and bibliography of eel larvae. The J.L.B. Smith Institute of Ichthyology, spec. publ., (7):121 pp.

Castle, P.H.J. 1970.

Distribution, larval growth, and metamorphosis of the eel Derichthys serpentinus Gill, 1884 (Pisces: Derichthyidae). Copeia, (3):444-452, I fig., 1 tab. 
Cassie, R. M. 1956.

Early development of the snapper Chrysophrys auratus Forster. Trans. R. Soc. N.z., 83:705-13, 36 fig., 4 pl., 2 tab.

Cassie, R. M. 1963.

Quantitative ecological studies of marine fish and plankton.

D.Sc. thesis, University of Auckland, New Zealand.

Ciechomski, J.Dz. de and Weiss de Vigo. 1971.

The influence of the temperature on the number of vertebrae in the Argentine anchovy, Engraulis anchoita (Hubbs and Marini). J. Cons. perm. int. Explor. Mer, $34(1): 37-42,1$ fig., 2 tab.

Clarke, F. E. 1879.

On some new fishes. Txans. Proc. N.Z. Inst., 11:291-295, 1 pl.

Clemens, H. B. 1956.

Rearing larval scombrid fishes in shipboard aquaria. Calif.

Fish Game, $42(1): 69-79,2$ fig., 2 tab.

Coakley, A. 1964.

Iife history and general biology of Trachelochismus pinnulatus

(Forster) (Order Xenopterygii). Unpublished M.Sc. thesis, University of Canterbury, New Zealand.

Cohen, D. M. 1970.

How many recent fish are there? Proc. Calif. Acad. Sci., 38(17): $341-346,1 \mathrm{fig}$.

Colton, J. B., Honey, K. A. and Temple, R. F. 1961.

The effectiveness of sampling methods used to study the distribution

of larval herring in the Gulf of Maine. J. Cons. perm. int. Explor.

Mer, $26(2): 180-190,8$ fig., 2 tab.

Cuvier, G. and Valenciennes, M. A. 1836.

Histoire naturelle des poissons. Paris, 11: $506 \mathrm{pp}$.

Daget, J. 1964.

Le crâne des Téleostéens. Mêm. Mus. natn. Hist. nat. Paris Sex. A, $31(2): 163-341,53 \mathrm{fig}$.

Dakin, W. J. and Colefax, A. N. 1934.

The eggs and early larval stages of the Australian pilchard Sardinia neopilchardus (Steind.). Rec. Aust. Mus., 19(2):136-140, 4 fig., 1 pl. 
Dakin, W. J. and Colefax, A. N. 1940.

Plankton of the New South Wales coastal waters. Publs Univ. Sydney Zool. Dep. Monograph, I:215 pp., Fishes, pp. 201-204, 1 pl.

Darby, M. M. 1966.

The ecology of fishes in tidal rockpools, with a revision of the common littoral species Tripterygion nigripenne Cuv, and Val., 1836 (Triptexygiidae:Blennioidei:Teleostei). Unpublished M.Sc. thesis, University of Canterbury, New Zealand.

De Beer, G. R. 1937.

The development of the vertebrate skull. Oxford: Clarendon Press, $552 \mathrm{pp} ., 143 \mathrm{pl}$.

Delmonte, P. J., Rubinoff, I.and Rubinoff, R. W. 1968.

laboratory rearing through metamorphosis of some Panamanian gobies. Copeia, (2):411-412.

Delsman, H. C. 1923.

Fish eggs and larvae from the Java sea 2. Chirocentrus dorab

(Forsk.). Treubia, 3(1):38-46, 9 fig.

Detwylex, R. and Houde, E. D. 1970.

Food selection by laboratory-reared larvae of the scaled sardine Harengula pensacolae (Pisces, Clupeidae) and the bay anchovy Anchoa mitchilli (Pisces, Engraulidae). Mar. Biol., Berlin 7: $214-222,7$ fig., 2 tab.

Deubler, E. E.Jr. 1958.

A comparative study of the postlarvae of three flounders (Paralichthys) in North Carolina. Copeia, (2):112-116.

Deuel, D. G., Clark, J. R. and Mansueti, A. J. 1966.

Description of embryonic and early larval stages of bluefish,

Pomatomus saltatrix. Trans. Am. Fish. Soc., 95:264-271, 4 fig., I tab. Doak, W. 1972.

Fishes of the New Zealand region. Auckland: Hodder and Stoughton, $1.32 \mathrm{pp}, 48$ fig., $48 \mathrm{pl}$.

Dotu, Y.and Mito, S. 1955.

On the breeding-habits, larvae and young of a goby, Acanthogobius flavimanus (Temminck et Schlegel). Jap. J. Ichthyol., 4:153-161, 5 fig., 2 tab. 
Dovel, W. L. 1960.

Larval development of the oyster toadfish, Opsanus tau.

Chesapeake Sci., 1(3-4): 187-195, 13 fig., 1 tab.

Dovel, W. L. 1963.

Larval development of clingfish, Gobiesox strumosus, 4.0 to 12.0

millimeters total length. Chesapeake Sci., 4(4):161-166, 6 fig., 3 tab.

Dutt, S. and Rao, V. V. 1960.

On the breeding habits and early development stages of petroscirtes

bipunctatus Day. J. zool. Soc. India, 12(2):158-161, I fig.

Ege, V. 1953.

Paralepididae I. (Paralepis and Lestidium). Taxonomy, ontogeny, phylogeny and distribution. Dana Rep., (40): 1-184, 33 fig.

Ege, V. 1957.

Paralepididae II. (Macroparalepis). Taxonomy, ontogeny, phylogeny and distribution. Dana Rep., (43): 1-101.

Eisler, R. 1961.

Effects of visible radiation on salmonoid embryos and larvae. Growth, $25(4): 281-346$.

Elder, R. D. 1966.

Larval teleosts in the plankton of Wellington Harbour, unpublished M.Sc. thesis, Victoria University of Wellington, New Zealand.

Evans, H. E. 1948.

clearing and staining small vertebrates in toto, for demonstrating ossification. Turtox News, 16(2):42-47, 5 fig.

Fahy, W. E. 1964.

A temperature-controlled salt-water circulating apparatus for developing fish eggs and larvae. J. Cons. perm. int. Explor. Mer, $28(3): 364-384,1$ fig., 1 tab.

Fahy, W. E. 1972.

Influence of temperature change on number of vertebrae and caudal fin rays in Fundulus majalis (Walbaum). J. Cons. perm. int. Explor. Mer, $34(2): 21.7-231$, 1 fig., 6 tab.

Fish, M. P. 1932.

Contributions to the early life histories of sixty-two species of fishes from Lake Erie and its tributary waters. Bull. Bur. Fish. , Wash. , 47:293-398, 144 fig. 
Fraser, T. H. 1972.

Sorne thoughts about the teleostean fish concept - the Paracanthopterygii. Jap. J. Ichthyol., 19(4):232-242, 1 fig., 2 tab.

Friedrich, H. 1961.

Physiological significance of light in marine ecosystems.

Am. Assoc. Adv. Sci., Washington D.C., (67): 257-70.

Gaxside, E. T. 1966.

Developmental rate and vertebral number in salmonids.

J. Fish. Res. Bd Can., $23(10): 1537-51,1$ fig., 6 tab.

Gibson, R. N. 1969.

The biology and behaviour of littoral fish. Oceanogr. Mar. Biol.

Ann. Rev., (7):367-410, 5 fig., 3 tab.

Gill, T. N. 1893 .

A comparison of Antipodal faunas. Mem. natn. Acad. Sci., $6: 89-124$.

Goodrich, E. S. 1958.

Studies on the structure and development of vertebrates. New York:

Dover Publications Inc., I: 485 pp., 512 fig.

Gosline, W. A. 1968.

The suborders of perciform fishes. Proc. U.S. natn. Mus., 124(3647): 78 pp., 12 fig., 3 tab.

Gosline, W. A. 1970.

A reinterpretation of the teleostean fish order Gobiesociformes.

Proc. Calif. Acad. Sci., 38 (19):363-382, 7 fig.

Graham, D. H. 1937.

Pairing courtship and parental care among three N.Z. fishes.

Wellington: Whitcombe and Tombs, 40 pp., $13 \mathrm{pl}$.

Graham, D. H. 1939.

Breeding habits of the fishes of otago Harbour and adjacent seas.

Trans. Proc. R. Soc. N.Z., 69: 361-372, 7 pl.

Graham, D. H. 1953.

Treasury of New Zealand fishes. Wellington: A.H. and A.W. Reed, 404 pp., 153 fig.

Greenwood, P. H., Rosen, D. E., Weitzman, S. H. and Myers, G. S. 1966.

Phyletic studies of teleostean fishes with provisional classification

of Jiving forms. Bull. Am. Mus. nat. Hist., 131(4):339-456, 9 fig.,

32 charts. 
Gregory, W. K. 1933.

Fish skulls: A study of the evolution of natural mechanisms.

Trans. Amer. Phil. Soc., 23(2):75-481, 302 fig.

Griffin, L. T. 1926.

Descriptions of New Zealand fishes. Trans. Proc. N.Z. Inst., 56: $538-546,6 \mathrm{p} 1$.

Gunther, A. 1861 .

Catalogue of the fishes in the British Museum vol. III -

Acanthopterygii. London: Taylor and Francis, $586 \mathrm{pp}$.

Harrington, R. W. 1955.

The osteocranium of the American cyprinid fish, Notxopis bifrenatus, with an annotated synonymy of teleost skull bones. Copeia, (4): 267-290, 7 fig.

Heath, E. and Moreland, J. M. 1967.

Marine fishes of New Zealand. Wellington: A.H. \& A.W. Reed. 56 pp., 100 fig.

Hewitt, G. C. 1964.

A new species of Caligus (Copepoda) on a species of Tripterygion from New Zealand. Trans. R. Soc. N.Z., Zool.,5(10):123-30, fi.gs.

Hewitt, G. C. and Hine, P. M. 1972.

Checklist of parasites of New Zealand fishes and of their hosts.

N.Z. J1 mar. Freshwat. Res., $6(1 \& 2): 69-114$.

Hollister, G. 1934.

Clearing and dyeing fish for bone study. Zoologica, N.Y., 12(10):

89-100.

Hollistex, G. 1936.

Caudal skeleton of Bermuda shallow water fishes. I. order

Isospondyli: Elopidae, Megalopidae, Albulidae, Clupeidae,

Dussumieriidae, Engraulidae. Zoologica, N.Y., 21(4):257-299, 53 fig.,

1 tab.

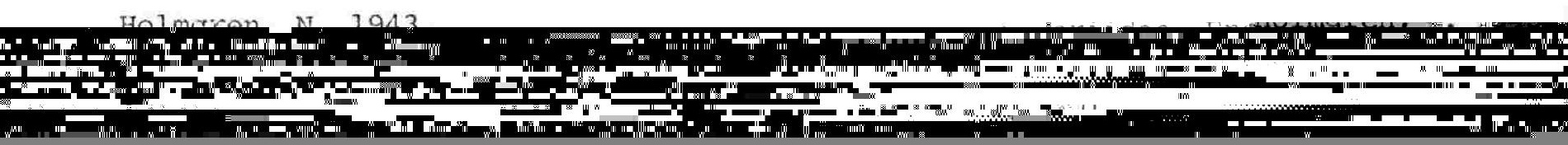


Houde, E. D. and Swanson, L. J. 1975.

Description of eggs and larvae of yellowfin menhaden, Brevoortia smithi. Fishery Bull. Fish Wildl. Serv. U.S., 73(3):660-673, 5 fig., 5 tab.

Hubbs, C. 1952.

A contribution to the classification of the blennioid fishes of the family Clinidae, with a partial revision of the Eastern Pacific forms. Stanford ichthyol. Bull., 4(2): 41-165, $64 \mathrm{fig}$.

Hubbs, C. 1953.

Revision and systematic position of the blenniid fishes of the genus Neoclinus. Copeia, (1):11-23, $16 \mathrm{fig}$.

Hubbs, C. 1958.

Dikellorhynchus and Kanazawaichthys: Nominal fish genera interpreted as based on prejuveniles of Malacanthus and Antennarius, respectively. Copeia, (4):282-285, 2 tab.

Hubbs, C. I. 1926.

The structural consequences of modifications of the developmental rate in fishes, considered in reference to certain problems of evolution. Am. Nat., 60(666):57-81.

Hubbs, C. L. 1943.

Terminology of early stages of fishes. Copeia, (4):260.

Hubbs, C. L. and Lagler, K. F. 1958.

Fishes of the great lakes region. Michigan: Cranbrook Institute of Science. 213 pp., 251 fig., 6 tab.

Hunter, J. R. 1972.

Swimming and feeding behaviour of larval anchovy Engraulis mordax. Fishery Bull. Fish Wildl. Serv. U.S., $70(3): 821-838,12$ fig., 4 tab. Hutton, F. W. 1872.

Fishes of New Zealand Catalogue with diagnosis of the species. Wellington: James Hughes, XV + 93 pp., 134 fig., $12 \mathrm{pl}$.

Hutton, F. W. 1873.

Contributions to the ichthyology of New Zealand.

Txans. Proc. N.Z. Inst. , 5:259-272, $7 \mathrm{pl}$.

Hutton, F. W. 1876.

Contributions to the ichthyology of New Zeal.and.

Trans. Proc. N.Z. Inst., 8:209-218.

Hutton, F. W. 1877.

Contributions to the ichthyology of New Zealand.

Trans. Proc. N.z. Inst., 9:353-354. 
Hutton, F. W. 1890 .

List of New Zealand fishes. Trans. Proc. N.Z. Inst., 22:275-285. Hutton, F. W. 1904.

Index Faunae Novae Zealandiae. London: Dulau \& Co., 372 pp. Jenyns, L. 1842.

The Zoology Voyage of H.M.S. Beagle during 1832-6. Part 4 . Fish. Smith, Elder, London, $151 \mathrm{pp}$.

Jillett, J. B. 1961.

Studies on the biology of Acanthoclinus quadridactylus (Bloch and Schneider). Published M.Sc. thesis, University of Auckland, New Zealand.

Jillett, J. B. 1968.

The biology of Acanthoclinus quadridactylus (Bloch and Schneider) (Teleostei-Blennioidea) II. Breeding and development. Aust. J. mar. Freshwat. Res., 19:9-18, 5 fig., 2 tab.

Johnson, R. K. and Barnett, M. A. 1975.

An inverse correlation between meristic characters and food supply in mid-water fishes: evidence and possible explanations. Fishery Bull. Fish Wildl. Serv. U.S., 73(2):284-298, 5 fig., 10 tab. Jollie, M. 1975.

Development of the head skeleton and pectoral girdle in Esox. J. moxph., $147(1): 61-88$, 11 fig., 1 tab.

Jones, S. 1950.

A note on the terminology of the early developmental stages of fish. J. zool. Soc. India, 2:39-41.

Jones, S. 1967.

On the terminology for phases and stages in the life history of teleostean fishes. Proc. zool. Soc. India, 20(1):99-102.

Kapoor, A. S. 1970.

Development of dermal bones related to sensory canals of the head in the fishes Ophicephalus punctatus Bloch (Ophicephalidae) and Wallago attu B1. \& Schn. (Siluridae). Zool. J. Linn. Soc., $49(2): 69-97,47$ fig., 4 pl.

Kendall, A. W. and Reintjes, J. W. 1975. Geographic and hydrographic distribution of Atlantic menhaden eggs and larvae along the middle Atlantic coast from RV Dolphin Cruises, 1965-66. Fishery Bull. Fish Wildl. Serv. U.S., 73(2): $317-335,13$ fig., 3 tab. 
Kjelson, M. A., Peters, D. S., Thayer, G. W. and Johnson, G. N. 1975. The general feeding ecology of postlarval fishes in the Newport River Estuary. Fishery Bull. Fish Wildl. Serv. U.S., 73 (1):137-144, 3 fig., 7 tab.

Lagler, K. F. 1964.

Freshwater fishery biology (2nd ed.). Iowa: Wm. C. Brown Company, $421 \mathrm{pp} ., 172 \mathrm{fig}$.

Laixd, M. 1951.

Studies on the Trypanosomes of New zealand fish. Proc. zool. Soc. Lond., 12l.(2):285-309, 1 fig., 2 tab., 5 pl.

Laixd, M. 1953.

The Protozoa of New Zealand intertidal zone fishes. Trans. R. Soc. N.z., $81(1): 79-143,2$ fig., 9 tab., 7 pl.

Lenarz, W. H. 1972.

Mesh retention of Larvae of Sardinops caerulea and Engraulis mordax by plankton nets. Fishery Bull. Fish Wildl. Serv. U.S., 70(3): 839-847, 7 fig., 2 tab.

Leonard, S. B. 1971.

Larvae of the fourspot flounder, Hippoglossina oblonga (Pisces: Bothidae), from the Chesapeake Bight, Western North Atlantic, Copeia, (4): 676-681, 2 fig.

Lindsey, C. C. 1954.

Temperature-controlled meristic variation in the paradise fish Macropodus opercularis (L). Can. J. Zool., 32(2):87-98.

Lindsey, C. C. and Ali, M. Y. 1965.

The effect of alternating temperature on vertebral count in the medaka (Oryzias latipes). Can. J. Zool., 43:99-104, 1 fig., 1 tab. Llewellyn, L. C. 1973.

Spawning, development, and temperature tolexance of the spangle perch, Madigania unicolor (Gunther), from inland waters in Australia. Aust. J. max. Freshwat. Res., 24(1):73-94, $13 \mathrm{fig}$.

Llewellyn, L. C. 1974.

Spawning, development and distribution of the Southern pigmy perch Nannoperca australis australis Ginther from inland waters in Eastern Australia. Aust. J. mar. Freshwat. Res., 25(1):121-149, 16 fig., 6 tab. 
Makushok, V. M. 1958.

The morphology and classification of the northern blennioid fishes (Stichaeoidae, Blennioidei, Pisces). Trudy. zool. Inst. Akad. Nauk. S.S.S.R., 25:3-129, (in Russian).

Mansueti, A. J. 1963.

Some changes in morphology during ontogeny in the pirate perch, Aphredoderus s. sayanus. Copeia, (3):546-557, 8 fig., 1 tab.

Mansueti, A. J. 1964.

Early development of the yellow perch, Perca flavescens.

Chesapeake Sci., 5(1-2):46-66, 16 fig., 1 tab.

Mansueti, A. J. and Hardy, J.D.Jr. 1967.

Development of fishes of the Chespeake Bay region, an atlas of egg, larval and juvenile stages, Part 1. Baltimore: Port City Press, 202 pp., $90 \mathrm{fig}$.

Mansueti, R. J. 1962.

Eggs, larvae and young of the hickory shad, Alosa mediocris, with cominents on its ecology in the estuary. Chespeake Sci., 3(3): 173-205, 12 fig., 8 tab.

Mansueti, R. J. 1964.

Eggs, larvae and young of the white perch, Roccus americanus, with comments on its ecology in the estuary. Chesapeake Sci., $5(1-2): 3-45,15$ fig., 9 tab.

Matsumoto, W. M. , Klawe, W. L., Richards, W. J. and Ueyanagi, S. 1966. Working party report on methods of collecting larvae. FAO Fish. Rep., (37):20-35, 3 fig., 3 tab.

May, R. C. 1970.

Feeding larval marine fishes in the laboratory: A review. Calif. Mar. Res. Comm., CalCOFI Rept., 14:76-83, 1 tab.

May, R. C. 1975.

Effects of temperature and salinity on fertilization, embryonic development, and hatching in Bairdjella icistia (Pisces: Sciaenidae), and the effect of parental. salinity acclimation on embryonic and larval, salinity tolerance. Fishery Bull. Fish Wildl. Serv. U.S., $73(1): 1-22,15$ fig., 11 tab.

McCulloch, A. R. and Phillipps, W. J. 1923. Notes on New Zealand fishes. Rec. Aust. Mus., 14:18-22, 1 fig., 1 pl. 
McCulloch, A. R. and Waite, E. R. 1918.

Some new and little-known fishes from South Australia.

Rec. S. Aust. Mus., $1(1): 1-78,6 \mathrm{p} 1$.

MCDowall, R. M. 1965.

Studies on the biology of the red-finned bully Gobiomorphus huttoni

(Ogilby) II - Breeding and life history. Trans. R. Soc. N.Z., Zool., $5(14): 177-196,6$ fig., 1 tab.

McKenzie, M. K. 1961.

Fisheries research in the East Cape area.

Fish. tech. Rep. N.z. mar. Dep., (I): 9 pp., 2 fig.

Miller, D., Colton, J. B. and Maxak, R. R. 1963.

A study of the vertical distribution of larval haddock.

J. Cons. perm. int. Explor. Mer, $28(1): 37-49,7$ fig., 3 tab.

Miller, J. M. and Sumida, B. Y. 1974.

Development of eggs and larvae of Caranx mate (Carangidae).

Fishery Bull. Fish Wildl. Serv. U.S., 72(2):497-514, 7 fig., 2 tab.

Mito, S. 1954.

Breeding habits of a blennioid fish, Salarias enosimae.

Jap. J. Ichthyol., 6:144-152, 4 fig., 3 tab.

Mito, S. 1963.

Pelagic fish eggs from Japanese watexs - VIII. Cottina.

Jap. J. Ichthyol., $11(3,6): 65-80,10 \mathrm{pl}$.

Moreland, J. M. and Dell, R. K. 1950.

Preliminary report on the ovoviviparity in a New Zealand

Blennioid fish Exicentrus rubrus (Hutton). N.Z. Sci. Rev.,

$8(3,4): 39-40,1 \mathrm{pl}$.

Morris, R. W. 1951.

Early development of the cottid fish Clinocottus recalvus (Greeley).

Calif. Fish Game, $37(3): 281-300,28 \mathrm{fig}$.

Morris, R. W. 1956.

Early larvae of four species of rockfish, Sebastodes.

Calif. Fish Game, 42(2):149-153, 2 fig., 2 tab.

Morris, R. W. 1965.

Seasonal changes in metabolism of four south temperate marine fishes.

Trans. R. Soc. N.Z., Zool., 6(15):141-152, 4 fig. 
Mosex, H. B. 1967.

Reproduction and development of Sebastodes paucispinis and comparison with other rock fishes off Southern California. Copeia, (4):773-797, 19 fig., 5 tab.

Moser, H. G. 1972.

Development and geographic distribution of the rockfish, Sebastes macdonaldi (Eigenmann and Beeson, 1893), Family Scorpaenidae, off Southern California and Baja California. Fishery Bull. Fish Wildl. Serv. U.S., 70(3):94l-958, 4 fig., 7 tab.

Moser, H. G. and Ahlstrom, E. H. 1970. Development of lantern fishes (family Myctophidae) in the California Current. Part I. Species with narrow-eyed larvae. Bu11. Los Angeles Cy Mus. Nat. Hist., Sci. 7:1-145, figs.

Moser, H. G. and Ahlstrom, E. H. 1972.

Development of the lantern fish Scopelopsis multipunctatus Brauer 1906, with a discussion of its phylogenetic position in the family Myctophidae and its role in a proposed mechanism for the evolution of photophore patterns in lantern fishes. Fishery Bull. Fish Wildl. Serv. U.S., 70(3):54l-564, 15 fig., 2 tab.

Moser, H. G. and Ahlstrom, E. H. 1974. Role of larval. stages in systematic investigations of marine teleosts: the Myctophidae, a case study. Fishery Bull. Fish Wildl. Serv. U.S., $72(2): 391-413,12$ fig., 2 tab.

Munro, I.S.R. 1955.

Eggs and larvae of the sabre-toothed oyster blenny, Dasson steadi (Whitley) (Blennidae). Aust. J. max. Freshwat. Res., 6(1):30-34, 2. Eig.

Murphy, G. I. and Clutter, R. I. 1972. Sampling anchovy laxvae with a Plankton purse seine. Fishery Bull. Fish Wildl. Serv. U.S., 70(3):789-798, 5 fig., 4 tab. New, D.A.T. 1966.

The culture of vertebrate embryos. London: Logos Press Iimited, 245 pp., 46 fig., 16 pl.

Norden, C. R. 1961.

The identification of laxval yellow perch, Perca flavescens and walleye, Stizostedion vitreum. Copeia, (3):282-288, 5 fig., 3 tab. 
Nordeng, H. and Bratland, P. 1971. Feeding of plaice (Pleuronectes platessa L.) and cod (Gadus morphua I.) 1.arvae. J.Cons.perm.int.Explor. Mer, 34(1):51-57, 2 fig. Norman, J. R. 1926.

The development of the chondrocranium of the eel (Anguilla vulgaris) with observations on the comparative morphology and development of the chondrocranium in bony fishes. Phil. Trans. R. Soc., ser. B , 214:369-464, $56 \mathrm{fig}$.

Orton, G. L. 1953a.

Development and migration of pigment cells in some teleost fishes. J. Morph., 93: 69-99, 5 pl.

Oxton, G. I. 1953b.

The systematics of vertebrate larvae. Syst. Zool., 2:63-75, 10 fig. Oxton, G. L. 1955.

Early developmental stages of the California barracuda, Sphyraena argentea Girard. Calif. Fish Game, 41(2):167-176, 2 fig. Orton, G. L. 1963.

Notes on larval anatomy of fishes of the order Lyomeri.

Copeia, (1):6-15, 4 fig.

Osse, J.W.M. 1.969.

Functional morphology of the head of the perch (Perca fluviatilis L.): an electromyographic study. Netherlands J. Zool., 19(3):289-392, 24 fig., 3 tab., 15 pl.

Patterson, C. 1975.

The braincase of pholjoophorid and leptolepid fishes, with a review of the actinopterygian braincase. Phil. Trans. R. Soc., ser. B, 269 (899):275-579, 151 fig.

Peckham, R. S. 1963.

Development of the caudal fin in the central mud minnow, Umbra limi (Kirtland). Copeia, (3):586-588, 5 fig.

Penrith, M. 1969. The systematics of the fishes of the family Clinidae in South Africa. Ann. S. Afr. Mus., $55(1): 121$ pp., 48 fig., 7 tab. Potthoff, T. 1974 .

Osteological development and variation in young tunas, genus Thunnus (Pisces, Scombridae), from the Atlantic Ocean. Fishery Bull. Fish Wildl. Serv. U.S., $72(2): 563-588,13$ fig., 13 tab. 
Potthoff, T. 1975.

Ichthyoplankton. Report on the CICAR ichthyoplankton workshop Scombrid, xiphiid and istiophorid taxonomy. Unesco technical papers in marine science, (20):22-23.

Quast, J. C. 1965.

Osteological characteristics and affinities of the hexagramid fishes, with a synopsis. Proc. Calif. Acad. Sci., 31(21):563-600, 3 fig., 2 tab.

Raitt, D.F.S. 1964 .

Scottish redfish larval investigations in 1962 with some observations on mid-oceanic echo-traces. J. Cons. perm. int. Explor. Mer, $29(1): 65-72,4$ fig. , 3 tab.

Rass, T. S. 1946.

Phases and stages in the ontogenesis of teleostean fishes. zool. J. Moscow, 25:137-148, 3 fig., (Russian, with English sumnary). Regan, C. T. 1913.

The classification of the percoid fishes. Ann. Mag. nat. Hist., ser. 8, 12:111-145.

Regan, C. T. 1914-16.

Larval and post-larval fishes. Br. Antarct. Terxa Nova Exped. 1910 Z00logy, I (4):125-156, $10 \mathrm{pl}$.

Rendahl, H. 1926.

Fishes from New Zealand and the Auckland and Campbell Islands. Vidensk. Meddr Dansk naturh. Foren., 81:1-16.

Richards, S. W. and McBean, A. M. 1966.

Comparison of postlarvae and juveniles of Fundulus heteroclitus and Fundulus majalis (Pisces:Cyprinodontidae). Trans. Am. Fish. Soc., 95:218-226, 4 fig.

Riley, J. D. and Thacker, G. T. 1963.

Marine fish culture in Britain III. Plaice (Pleuronectes platessa (L)) rearing in closed circulation at Lowestoft, 1961. J. Cons. perm. int. Explor. Mer, $28(1): 80-90,10 \mathrm{fig.,} 1$ tab.

Risso, A. 1826.

Histoire naturelle des principales productions de l'Europe meridionale et paxticulierement de celles des envicons de Nice et des Alpes maxitimes. Vol. 3. Paris \& Strasbourg, pl. 
Ritchie, L. D. 1969.

Aspects of the biology of the butterfish Coridodax pullus (Forster). Unpublished M.Sc. thesis, Victoria University of Wellington, New Zealand.

Roberts, P. E. 1968.

Campbell Island plankton studies. Published M.Sc. thesis, Victoria University of Wellington, New Zealand.

Roberts, T. R. 1966.

Description and osteology of Lepidarchus adonis, a remarkable new characid fish from West Africa. Stanford ichthyol. Bull., 8(3): 209-227, 11 fig.

Roberts, T. R. 1.969.

Osteology and relationships of characoid fishes, particularly the genera Hepsetus, Salminus, Hoplias, Ctenolucius, and Acestrorhynchus. Proc. Calif. Acad. Sci., 35(15):391-500, $60 \mathrm{fig}$.

Robextson, D. A. 1973.

Planktonic eggs and larvae of some New zealand marine teleosts. Unpublished Ph.D. thesis, Otago University, Dunedin, New Zealand. Robertson, D. A. 1975.

Planktonic stages of the teleost family Carapidae in eastern New Zealand waters. H.Z. JI mar. Freshwat. Res., 9(3):403-409,

2 fig., 1 tab.

Robertson, D. A. and Raj, U. 1971.

Egg and yolk sac stages of the sand flounder (Teleostei:

Pleuronectidae). N.Z. Jl mar. Ereshwat. Res., 5(3\& 4): 404-414,

5 fig. , 3 tab.

Robins, C. R. and Randall, J. E. 1965.

Three new Western Atlantic fishes of the blennioid genus Chaenopsis, with notes on the related Iucayablennius zingaxo. Proc. Acad. nat. Sci. Philad., $117(6): 213-227,4$ fig.

Rosen, D. E. 1973.

Interclationships of higher euteleostean fishes.

J. Linn. Soc. , 53:397-513, 129 fig., 4 tab.

Rosenblatt, R. H. and Parx, T. D. 1969.

The Pacific species of the clinid fish genus Paxaclinus. Copeia, (1): I-20, 7 fig., 1 tab. 
Ruck, J. G. 1968.

A study of co-existence between two inter-tidal blennies. Unpublished B.Sc. (Hons) project.

Ruck, J. G. 1971.

Development of the Iumpfish Trachelochismus melobesia (Pisces:

Gobiesocidael. Zool. Publs Vict. Univ. Wellington, (57):1-9, 4 fig.

Ruck, J. G. 1973 a.

Development of Tripterygion capito and T. robustum (Pisces:

Triptexygiidae). Zool. Publs Vict. Univ. Wellington, (63):1-10, 3 fig.

Ruck, J. G. 1973b.

Development of the clingfishes, Diplocrepis puniceus and

Trachelochismus pinnulatus (Pisces: Gobiesocidae). Zool. Publs Vict. Univ. Wellington, (64):1-12, 4 fig.

Ruck, J. G. (in press a).

The early development of Forsterygion varium and Gilloblennius

decemaigitatus (Pisces: Tripterygiidae). N.Z. J1 max. Freshwat. Res.

Ruck, J. G. (in press b).

Development of Gilloblennius tripennis (Pisces: Tripterygiidae).

N.Z. Il mar. Freshwat. Res.

Ruck, J. G. 1975.

A light-trap for collecting marine fish larvae and invertebrates. Tuatara, $21(3): 98-104,5$ fig.

Rugh, R. 1948.

Experimental embryology. Minneapolis: Burgess Publishing Company, $481 \mathrm{pp}$.

Runyan, S. 1961.

Early development of the clingfish, Gobiesox strumosus Cope. Chesapeake Sci., $2(3,4): 113-141,33$ fig., 6 tab.

Ryland, J. S. 1966.

Observations on the development of larvae of the plaice,

Pleuronectes platessa $\mathrm{L}$., in aquaria. J. Cons. perm. int. Explor. Mer, $30(2): 177-195,10$ fig., 5 tab.

Saville, A. 1959.

Mesh selection in plankton nets. J. Cons. perm. int. Explox. Mer, $23: 192-201$. 
Schultz, L. P., Chapman, W. M., Lachner, E. A. and Woods, L. P. 1960. Fishes of the Maxshall and Marianas Islands. U.S. natn. Mus. Bull., $202(2): 438$ pp., 132 fig., 119 tab., $47 \mathrm{pl}$.

Shelbourne, J. E. 1956.

The effect of water conservation on the structure of maxine fish enbryos and larvae. J. mar. biol. Ass. U.K., 35(1):275-286.

Shelbourne, J. E. 1963.

Marine fish culture in Britain II. A plaice rearing experiment at Port Erin, Isle of Man, during 1960, in open sea water circulation. J. Cons. perm. int. Explor. Mer, 28 (1):70-79, 9 fig., 2 tab.

Shelbourne, J. E. 1964.

Experimental sea-water systems for rearing fish larvae. Fishery Bull. Fish Wildl. Serv. U.S., Rep., 63:81-93, $8 \mathrm{fig}$.

Shelbourne, J. E., Riley, J. D. and Thacker, G. T. 1963.

Marine fish culture in Britain I. Plaice rearing in closed

circulation at Lowestoft, 1957-1960. J. Cons. perm. int. Explor. Mer, $28(1): 50-69$, 10 fig., 1 tah.

Smith, C. L. and Bailey, R. M. 1962.

The subocular shelf of fishes. J. morph., 110:1-17, 3 pl. Smith, D. G. 1975 .

Ichthyoplankton. Report of the CICAR ichthyoplankton workshop Leptocephali taxonomy. Unesco technical papers in marine science, (20) $: 19-20$.

Smith, P. E. 1972.

The increase in spawning biomass of northern anchovy, Engraulis mordax. Fishery Buil. Fish Wildl. Serv. U.S., 70(3):849-874, 13 fig., 12 tab.

Smith, P. E., Counts, R. C. and Clutter, R. I. 1968.

Changes in filtering efficiency of plankton nets due to clogging

under tow. J. Cons. perm. int. Explor. Mer, 32(2):232-248, 6 fig., 4 tab.

Spxinger, V. G. 1955.

The taxonomic status of the fishes of the genus stathmonotus, including a review of the Atlantic species. Bull. mar. Sci. Gulf. Caribb., 5 (1955):66-90, 2 fig., 2 tab.

Springer, V. G. 1958.

systematics and zoogeography of the clinid fishes of the subtribe

Labrisomini Hubbs. Publs Inst. mar. Sci. Univ. Tex., 5:418-491. 
Springer, V. G. 1964.

A review of: A revised classification of the blennioid fishes of the American family Chaenopsidae. By J. S. Stephens, Jx. 1963. Copeia, (3):591-593.

Springer, V. G. 1966.

Medusablennius chani a genus and species of blennioid fish from Tuamotu Archipelago: Its implication on blennioid classification.

Copeia, (1):56-60, 3 fig.

Springer, V. G. 1968.

Osteology and classification of the fishes of the family Blenniidae. U.s. natn. Mus. Bull., 284:85 pp., $16 \mathrm{fig.,} 11 \mathrm{pl}$.

Springer, V. G. 1971.

Revision of the fish genus Ecsenius (Blenniidae, Blenniinae,

Salariini). Smithson. Contr. Zool., (72): 74 pp., $36 \mathrm{fig}, 18$ tab. Springer, V. G. 1972.

Synopsis of the tribe Onobranchini with descriptions of three new genera and two new species (Pisces: Blennidae). Smithson. Contr. Zool., (130): 31 pp., 6 tab.

Springer, V. G. and Freihofer, W. C. (in press).

study of the monotypic fish family pholidichthyidae.

Springer, V. G. and Gomon, M. F. 1975.

Revision of the blenniid fish genus Omobranchus with descriptions of three new species and notes on other species of the tribe Onobranchini. Smithson. Contr. Zool., (177):135 pp., 52 fig., 17 tab. Stephens, 'J. S. 1963.

A revised classification of the blennioid fishes of the American family Chaenopsidae. Univ. Calif. Publs Zool., 68:165 pp., 11 fig., 24 tab., $15 \mathrm{pl}$.

Stephens, J. S. 1970.

Seven new chaenopsid blennies from the Western Atlantic. Copeia, (2): 28c-309, 15 fig., 11 tab.

Stephens, J. S. Jr and springer, V. G. 1973.

Clinid fishes of Chile and Peru, with description of a new species Myxodes ornatus, from Chile. Smithson. Contr. Z0ol., (159):24 pp., 15 fig., 3 tab. 
Swarup, H. 1958.

Stages in the development of the stickleback Gasterosteus

aculeatus (L). J. Embryol. exp. Morph. 6(3):373-383, $31 \mathrm{fig}$.

Taning, A. V. 1946.

Stage of determination of vertebrae in teleostean fishes.

Nature, Lond. , 157:594- -595, 1 fig.

Taylor, W. R. 1967.

An enzyme method of clearing and staining small vertebrates.

Proc. U.S. natn. Mus., $122(3596): 1-17$.

Tavolga, W. N. 1956.

Visual, chemical and sound stimuli as cues in the sex discriminatory behaviour of the gobiid fish Bathygobius soporator. Zoologica, N.Y., $41(7): 49-65$, I fig., 3 tab.

Tavolga, W. N. and Rugh, R. 1947.

Development of the platŷfish, Platypoecilus maculatus. Zoologica,

N.Y., $32: 1-15,25$ fig.

Thomson, G. M. 1897.

[On the establishment of a fish-hatching station]. Trans. Proc.

N.z. Inst., 29:626.

Thomson, G. M. 1898 (a) .

Notes on New Zealana fisheries [with discussion of proposal to

establish a fish-hatchery at purakanui]. Trans. Proc. N.Z. Inst.,

$30: 576-80$.

Thomson, G. M. 1898 (b).

The proposed biological station and fish-hatchery near Dunedin.

Proc. Aust. Ass. Adv. Sci.., 7:576.

Thomson, G. M. 1905.

The Portobello marine fish-hatchery and biological station.

Trans. Proc. N.Z. Inst., 38:529-558, 5 tab., 5 pl.

Thomson, G. M. and Anderton, T. 1921.

History of the Portobello Marine Fish-hatchery and Biological Station. Bull. Bd Sci. Art. iv.Z., (2):131 pp.

Thomson, J. M. and Bennett, A. E. 1953.

The oyster blenny, Omobranchus anolius (Valenciennes) Blenniidae.

Aust. J. mar. Ereshwat. Res., 4(2):227-233, 3 fig., 2 tab. 
Tibbo, S. N., Scattergood, L. W. and Temple, R. F. 1958.

on the occurrence and distribution of larval herring (Clupea

harengus L.) in the Bay of Fundy and the Gulf of Maine. J. Fish. Res. Bd Can., 15(6):1451-1469, 9 fig., 4 tab.

Topp, R. W. and Cole, C. F. 1968.

An osteological study of the sciaenid genus, Sciaenops Gill

(Teleostei, Sciaenidae). Bull. mar. Sci. Gulf Caribb., 18(4):902-945,

13 fig., 1 tab.

Tyler, J. C. 1963.

The apparent reduction in number of precaudal vertebrae in trunkfishes (Ostraciontoidea, Plectognathi). Proc. Acad. nat. Sci., Philad., $115(7): 153-190,13 \mathrm{fig}$.

Waite, E. R. 1913.

Fishes of the genus Tripterygion and related genera in New Zealand. Rec. Canterbury Mus., $2(1): 1-16,5$ pl.

Webb, B. F. 1973.

Fish populations of the Avon-Heathcote Estuary, 2. Breeding and gonad maturity. N.Z. JI mar. Freshwat. Res., 7(1 \& 2): 45-66, 26 tab. Weisel, G. F. 1947.

Breeding behaviour and early development of the mudsucker, a gobiid fish of California. Copeia, 2:77-85, $10 \mathrm{fig.,} 1 \mathrm{tab}$. Weitzman, S. H. 1954.

The osteology and the relationships of the South American characid fishes of the subfamily Gasteropelecinae. Stanford ichthyol. Bull., $4(4): 213-263,18$ fig., 2 tab.

Weitzman, S. H. 1962.

The osteology of Brycon meeki, a generalized characid fish, with an osteological definition of the family. Stanford ichthyol. Bull., $8(1)$ : $77 \mathrm{pp} ., 21 \mathrm{fig}$.

Whitley, G. P. 1930.

Ichthyological miscellanea. Mem. Qd Mus., 10: 8-31, $1 \mathrm{fig.,} 1$ pl. Whitley, G. P. 1968 .

A checklist of the fishes recorded from the New zealand region.

Aust. Zool., 15(1):100 pp.

Whitley, G. P. and Phillipps, W. J. 1940.

Descriptive notes on some New Zealand fishes. Trans. Proc. R. Soc. N.Z., $69(2): 228-236,2 \mathrm{pl}$. 
Winn, H. E. and Miller, R. R. 1954. Native postlarval fishes of the Lower Colorado River Basin, with a key to their identification. Calif. Fish Game, 40(3):273-285, 1 fig., 1 tab., 4 pl.

Woolcott, W. S., Beirne, C. and Hall, w. M. 1968.

Descriptive and comparative osteology of the young of three species of flounders, genus Paralichthys. Chesapeake Sci., 9(2):109-120, 4 fig., 8 tab.

Young, N. W. 1927. The spawning of the seahorse. N.Z. J1 Sci., 8:289. 
An annotated bibliography of New zealand teleost eggs and larvae, up to the end of 1972

Works included here have information relating to either the embryological, prolarval, larval, post-larval and prejuvenile stages of marine and freshwater teleosts of New Zealand. Numerous articles contain fragmentary information on breeding habits and spawning times but are not included here, unless they in some way describe the above stages. Genera and species in the annotations are as they appear in the publications to which they refer.

However, the current names are used in the checklist preceeding the main bibliography. Definitions of various developmental stages (prolarvae etc.) follow those used by Mansueti and Hardy (1967) which are adapted from Hubbs (1943, 1958).

The main sources of references are:

Phillipps, W. J. 1927. Bibliography and index of New Zealand fishes. Fish. Bull. N.Z. max. Dep., (1): $68 \mathrm{pp}$.

Freed, D. 1963. Bibljography of New Zealand marine zoology, 1769-1899. Mem. N.Z. oceanogr. Inst., 16: 46pp., plates.

McDowall, R. M. 1964. A bibliography of the indigenous freshwater fishes of New Zealand. Trans. R. Soc. N.Z., 5(1):1-38. Whitley, S. P. 1968. A check-list of the fishes recorded from the New Zealand region. Aust. Zool., 15(1):1-102.

Relevant literature was also found amongst the cited references of many articles.

The leptocephali of eels are excluded as these are adequately covered by Castle (1969). Interest in the early developmental stages of marine fish increased in New Zealand toward the end of the last century, possibly as a result of the establishment of hatcheries in Norway and Britain (Atz, 1964; Shelbourne, 1964). The purpose of these was to replenish depleted fish stocks in the North Sea. Thomson (1897, 1898a, 1898b) proposed that a fish-hatchery be built at Purakanui near Dunedin. The main purpose of this hatchery was to study the life history of local 
fish, replenish the local fish populations and to introduce and rear "desirable" species of foreign fish such as the turbot Rhombus maximus and the herring Clupea harengus L. After an initial flourish (Thomson, 1905; Anderton, 1906) the output of data on egg and larval fish throughout New Zealand declined, possibly comparable to a similar decline in activity by overseas hatcheries (Shelbourne, 1964). In recent years there has been a steady increase in developmental research in New zealand and overseas, reflecting the growing importance of this knowledge in fisheries research and management. A similar trend, although less marked, is followed by developmental research of freshwater fishes. Emphasis has been on commercial fish such as the whitebait, eels and trout.

\section{Checklist}

Acanthoclinus guadridactylus (Bloch and Schneider, 1801): 14, 16, 19, 20, 30. Cantherines scaber (Bloch and Schneider, 1801): 26.

Cantherines convexirostris (Gunther, 1870): 14 .

Caulopsetta scapha (Bloch and Schneider, 1801): 1.

Centrolophus maoricus Ogi.1by, 1893: 26 .

Cheilodactylus macropterus (Bloch and Schneider, 1801): 21, 30, 36 .

Chelidonichthys kumu (Lesson and Garnot, 1829): 1, 22, 35.

Chrysophrys auratus (Bloch and Schneider, 1801): 6, 7, 18, 30 .

Colistium guntheri. (Hutton, 1873): 35.

Colistium nuäipinnis (Waite, 1910): 34 .

Congiopodus leucopaecilus (Richardson, 1846): 30, 34, 35.

Contusus richei (Frominville, 1813): 14.

Coridodax pullus (Bloch and Schneider, 1801): 27.

Cubiceps caeruleus Regan, 1914: 26.

Dellichthys morelandi Briggs, 1955: 14.

Diodon sp.: 26.

Diaphus sp.: 26.

Diplocrepis puniceus (Richardson, 1846): 16,33 .

Engraulis australis (White, 1790): 4,14.

Ericentrus rubrus (Hutton, 1872): 23.

Forstexygion capito (Jenyns, 1842): 16, 26, 32.

Eorsterygion nigripenne (Valenciennes in Cuvier and Valenciennes, 1836): 32. 
Galaxias argenteus (Gmelin, 1789): 62, 64, 66, 69, 80, 81.

Galaxias brevipennis Gunther, 1866: 41, 62, 64, 69, 80, 81.

Galaxias burrowsius (Phillipps, 1926): 17.

Galaxias divergens Stokell, 1959: 58, 64, 80 .

Galaxias fasciatus Gray, 1842: 41, 64, 69, 80, 81.

Galaxias maculatus (Jenyns, 1842): 39, 40,41, 42, 43, 44, 45, 47, 49, 50,

$51,52,53,54,55,56,57,59,60,62,64,67,68,69,70,71,73$,

$74,75,76,78,79,80,81$.

Galaxias postvectis Clarke, 1899: 41, 69, 80, 81 .

Galaxias vulgaris stokell, 1949: 39, 43, 64, 70.

Gastrocymba quadriradiata (Rendahl, 1925): 28, 29.

Gilloblennius decemdigitatus (Clarke, 1879): 14.

Gobiomorphus gobioides (Cuvier and Valenciennes, 1837): 48, 75 .

Gobiomorphus huttoni (Ogilby, 1894): 61, 65.

Grahamichthys radiatus (Cuvier and Valencicnnes, 1837): 14 .

Haplophryne mollis Regan, 1916: 26.

Helcogramia medium (Gunther, 1861): 14.

Helicolenus papillosus (Bloch and Schneider, 1801): 14, 16, 35.

Hemirhamphus intermedius Cantor, 1842: 16.

Hippocampus abdominalis Lesson, 1818: 14, 16, 38.

Ichthyocampus filum Gunther, 1870: 14.

Lampanyctus Iongipinnis Regan, 1916: 26.

Lampanyctus macropterus Brauer, 1916: 26.

Lepidopus caudatus (Euphrasen, 1788): 26.

Lepidotrigla brachyoptera Hutton 1872: 35 .

Limnichthys fasciatus Waite, 1904: 26.

Myctophum coccoi Cocco, 1829: 26.

Neochanna apoda Gunther, 1867: 46.

Neophrynichthys latus (Hutton, 1876): 16.

Notoclinus compressus (Hutton, 1872): 14.

Paxaperci.s colias (Bloch and Schneider, 1801): 2, 25, 34, 35.

Peltorhamphus novaezeelandiae (Gunther, 1862): 10, 35 .

Prototroctes oxyrhynchus Gunther, 1870: 49.

Prymnothonus sp.: 26.

Pseudolabrus celidotus (Bloch and Schneider, 1801): 8, 14 .

Retropinna sp.: 49.

Retropinna anisodon Stoke11, 1941: 72, 
Retropinna retropinna (Richardson, 1848): 75 .

Rhombosolea leporina Gunther, 1873: 10.

Rhombosolea plebeia (Richardson, 1843): 1, 10, 17, 30, 35 .

Rhombosolea tapirina Gunther, 1862: 28, 29, 35.

Sardinops neopilchardus (Steindachner, 1879): 3, 4, 11, 12, 14, 26.

Scombresox forsteri. Cuvier and Valenciennes, 1846: 26.

Scorpaena cardinalis (Richardson, 1842): 14 .

Scorpis violaceus (Hutton, 1873): 26 .

Sprattus antipoduin (Hector, 1842): 3, 4, 5 .

Stigmatophora longirostris Hutton, 1872: 14 .

Thyrsites atun (Euphrasen, 1791): 26.

Trachelochismus melobesia Phillipps, 1927: 14, 31.

Trachelochismus pinnulatus (Bloch and Schneider, 1801): 9, 14, 33. 


\section{Marine larvae}

1. Anderton, T.1906. Observations on New Zealand fishes, etc., made at the Portobello Marine Fish-hatchery. Trans. Proc. N.Z. Inst., 39:477-96.- The embryological and early Iarval development of Trigla kumu Lesson and Garnot, 1826, Caulopsetta scapha (Forster, 1801) and Rhombosolea plebeia (Richardson, 1843) are described and figured.

2. Anderton, T. 1910. Marine Fish Hatchery, Portobello. Marine Department (Annual Report for 1909-10). (H.15):9-12, 1 pl., contained in Appendix to the Journals, H. of R. New Zealand. III, H-I.-Tabled data on the release of axtificially spawned fish larvae. Colour plate of the eggs and prolarvae of the blue cod, Parapercis colias (Forster, 1801) but no descriptive information.

3. Baker, A. N. 1971. Reproduction, early life history, and age-growth relationships of the New Zealand pilchard, Sardinops neopilchardus (Steindachner). Published Ph.D. thesis, Victoria University of Wellington, New Zealand.-Has information on the egg development of Sprattus antipodum (Hector, 1872).

4. Baker, A. N. 1972. Reproduction, Early life history, and age-growth relationships of the New Zealand Pilchard, Sardinops neopilchardus (Steindachner). Eish. Res. Bu11. N.z., (5): 64pp., 39 fig., 9 tab.Thorough study on the development of the eggs and larvae through to the juvenile. Includes compaxative data on the post-larvae of s. neopilchardus (Steindachner, 1879) Engraulis australis (White, 1790) and Sprattus antipodum.

5. Baker, A. N. 1973. Spawning and development of the New Zealand sprat, Sprattus antipodum (Hector). Zool. Publs Vict. Univ. Wellington, (62) : 12pp., $18 \mathrm{fig.-The} \mathrm{late} \mathrm{embryological,} \mathrm{prolarval} \mathrm{through} \mathrm{to} \mathrm{the}$ adult stages are described and figured in detail.

6. Cassie, R. M. 1956. Early development of the snapper Chrysophrys auratus Forster. Irans. R. Soc. N.Z., 83:705-13, 36 fig., 4 pl., 2 tab.Describes in detail the embryological stages and the development of the $3 \mathrm{~mm}$ prolarva. Good photographic series.

7. Cassie, R. M. 1963. Quantitative ecological studies of marine fish and plankton. D.Sc. thesis, University of Auckland, New Zealand.Contains information from the 1956 publication. 
8. Choat, J. H. 1962. Studies in the genus Pseudolabrus (Pisces). Unpublished M.Sc. thesis, Victoria University of Wellington, New zealand.-Describes in detail the colour patterns of prejuvenile $(15,18,22$ \& $33 \mathrm{~mm}$ s.1.) Pseudolabrus celidotus (Forstex, 1801).

9. Coakley, A. 1964. Life history and general biology of Trachelochismus pinnulatus (Forster) (Order Xenopterygii). Unpublished M.Sc. thesis, University of Canterbury, New Zealand.-Description of egg masses (egg size) spawning and the prolarva.

10. Colman, J. A. 1973. Spawning and fecundity of two flounder species in the Hauraki Gulf, New Zealand. N.Z. J1 mar. Freshwat. Res., 7 ( 1 \& 2):2l-43, 8 fig., 9 tab.- Outlines the problems involved in identifying the fertilized eggs of Rhombosolea plebeia (Richardson, 1843), R. leporina Gunther, 1873 and Peltorhamphus novae-zeelandiae Gunthex, 1862.

11. Dakin, W. J. and Colefax, A. N. 1934. The eggs and early larval stages of the Australian pilchard - Sardinia neopilchardus (Steind.). Rec. Aust. Mus., 19(2):136-140, 4 fig., 1 pl.- Egg development outlined briefly. Nore complete information on the larvae and postlarvae.

12. Dakin, W. J. and Colefax, A. N. 1940. Plankton of the New South Wales Coastal waters. Publs. Univ. Syaney Zool. Dep. Monograph, 1:215pp., Fishes, pp. 201-204, l pl.- An abbreviated repetition of their 1934 paper.

13. Darby, M. M. 1966. The ecology of fishes in tidal rockpools, with a revision of the common littoral species Tripterygion nigripenne Cuviex and Valenciennes, 1836 (Tripterygiidae:Blennioidei: Teleostei). Unpublished M.Sc. thesis, University of Canterbury, New Zealand.- Brief description of the eggs and prolarvae of Hel.oogramma medium (Ginther, 1861).

14. Elder, R. D. 1966. Larval teleosts in the plankton of Wellington Harbour. Unpublished M.Sc. thesis, Victoria University of Wellington, New Zealand.- Various larval stages of 20 species ( 3 unidentified) are figured and described.

Engraulis australis (White, 1790) Sardinops neopilchardus (Steindachner, 1879). Ichthyocampus filum Gunther, 1870 Stighatophora lorgicostris Hutton, 1872 
Hippocampus abdominalis Lesson, 1818

pseudolabrus celidotus (Forster, 1801)

Grahamichthys radiatus (Cuvier and Valenciennes, 1837)

Acanthoclinus quadridactylus (Bloch and Scheider, 1801)

Tripterygium decendigitatus Clarke, 1879

Notoclinus compressum (Hutton, 1872)

Helicolenus papillosus. (Bloch and Schneider, 1801)

Scorpaena cardinalis Richardson, 1842

Dellichthys morelandi Briggs, 1955

Trachelochismus melobesia Phillipps, 1927

Trachelochismus pinnulatus (Forster, 1801)

Novodon convexirostris (Gunther, 1870)

Sphoeroides richei (Freminville, 181.3)

15. Grahar, D. H. 1937. Pairing, courtship and parental care among three N.z. fishes. Wellington: Whitcombe and Tombs, 40pp., $13 \mathrm{pl}$.

16. Grahar, D. H. 1939. Breeding habits of the fishes of otago harbour and adjacent seas. Trans. Proc. R. Soc. N.Z., 69: 361-372, $45 \mathrm{fig.,} 7 \mathrm{pl}$ - Includes very brief descriptions of the egg clusters, eggs and larvae. Drawings in general unclear. Hippocampus abdominalis Lesson, 1818 Hemirhamphus intermedius Cantor, 1842 Acanthoclinus quadridactylus (Bloch and schneider, 1801) Tripterygion varjum (Forster, 1801)

Helicolenus percoides (Richardson, 1.845)

Diplocrepis puniceus. (Richardson, 1846)

Neophrynichthys 1.etus futton, 1876

17. Graham, D. H. 1953. A treasury of New Zealand fishes. Welington: A.H. \& A.W. Reed, $404 \mathrm{pp} ., 1.53 \mathrm{fig} .-T h e$ work of 1939 is repeated, with an added description of the larval development of Rhombosolea plebeia (Richardson, 1843).

18. Hefford, A. E. 1929. Report on the fisheries of the Hauraki Gulf, with special reference to the snapper fishery and to the effects of "Power" fishing (Trawling and Danish-seining). Rep. Fish. N.Z. 1929: 30-55.- Brief reference to spawning, eggs and larvae. 
314.

19. Jillett, J. B. 1961. Studies on the biology of Acanthoclinus quadridactylus (Bloch and Schneider). Published M.Sc. thesis, University of Auckland, New Zealand.- A brief description of the eggs and embryological development. Early larval development to $5.5 \mathrm{~mm} \mathrm{s.1.} \mathrm{is} \mathrm{described} \mathrm{and} \mathrm{figured} \mathrm{in} \mathrm{detail.}$

20. Jillett, J. B. 1968. The biology of Acanthoclinus quadridactylus (Bloch and Schneider) (Teleostei-Blennioidea) II. Breeding and development. Aust. J. mar. Freshwat. Res., 19: 9-18, 5 fig., 2 tab.- Material contained in the thesis.

2]. McKenzie, M. K. 1961. Fisheries research in the East Cape area. Fish. tech. Rep. N.Z. mar. Dep., (1): 9pp., 2 fig.- The development of Cheilodactylus macropterus (Forster, 1801) from the 1 cell stage to 8 days after hatching is figured and briefly described. Information on artificial fertilization and the effect of temperature on development is included.

22. Mito, S. 1963. Pelagic fish eggs from Japanese waters - VIII. Cottina. Jap. J. Ichthy., $11(3,6): 65-80,10 \mathrm{pl} .$, in Japanese with English sumaxy.- Includes brief descriptions and drawings of the embryological and early larval stages of Chelidonichthys kumu (Lesson and Garnot, 1826).

23. Moreland, J. M. and Dell, R. K. 1950. Preliminary report on the ovoviviparity in a New Zealand Blennioid fish Exicentrus rubrus (Hutton). N.Z. Sci. Rev., 8(3-4): 39-40, 1 pl..- Notes about the embryological development which occurs within the abdomen of the adult female.

24. Rapson, A. M. 1940. The reproduction, growth and distribution of the lemon soles (Pelotretis flavilatus Waite) of Tasman Bay and Marlboxough Sounds. Fish. Bull. N.z. mar. Dep., 7:56 pp., 16 fig., 21 tab.- Deals with egg and larval distribution, and aspects of embryological, larval and post-larval stages.

25. Rapson, A. M. 1956. Biology of the blue cod (Parapercis colias, Forstex) of New Zealand. Unpublished D.Sc, thesis, Victoria University of Wellington, New Zealand.- Brief description of larval and post-larval characteristics. Larvae figured, but no size scale given.

26. Regan, C. T. 1914-16. Larval and post-larval fishes. Br. Antarct. Texra Nova Exped., 1910 Zoology, I (4):125-156, 10 pl. Regan identifies and figures various larval, post-larval and juvenile stages of 18 species from around the Northem coast of New Zealand. 
Sardina neopilchardus (Steindachner, 1879)

Prymnothonus sp.

Myctophum coccoi Cocco, 1829

Diaphus $\mathrm{sp}$.

Lampanyctus macropterus Brauer, 1916

I. longipinnis Regan, 1916

Anguilla australis Richardson, 1841

Scombresox forsteri Cuvier and Valenciennes, 1846

Scorpis violaceus (Hutton, 1873)

Limnichthys fasciatus waite, 1904

Cubiceps caeruleus Regan, 1914

Centrolophus maoricus Ogilby, 1893

Thyrsites atun (Euphrasen, 1791)

Lepidopus caudatus (Euphrasen, 1788)

Tripterygium varium (Forster, 180I)

Monacanthus scaber (Forster, 1801)

Diodon sp.

Haplophryne mollis Brauer, 1902

27. Ritchie, L. D. 1969. Aspects of the biology of the butterfish

Coridodax pullus (Foxster). Unpublished M.Sc. thesis, Victoria

University of Wellington, New Zealand.- A full account of the embryological and prolarval stages, with a good photographic series.

28. Roberts, P. E. 1968. Campbell Island plankton studies. Published M.Sc. thesis, Victoria University of Wellington, New zealand.- Notes the presence of the laxvae of Rhombosolea tapirina Gunther, 1862 and Gastrocymba quadriradiata (Rendahl, 1925) at Campbell Island. A photograph of each is included.

29. Roberts, P. E. 1972. The plankton of Perseverence Harbour, Campbell Island, New Zealand. Pacif. Sci., 26(3):296-309, 6 fig., 3 tab. Repetition of the thesis information, but without the photographs.

30. Robextson, D. A. and Raj, U. 1971. Egg and yolk sac stages of the sand flounder (Teleostei:Pleuronectidae). N.Z. Jl max. Freshwat. Res., $5(3 \& 4): 404-414,5$ fig., 3 tab.- A detailed description of the embryological and prolarval stages of Rhombosolea plebeia (Richardson, 1843) coupled with a photographic sexies. The eggs of 5 other species are compared with those of R. plebeia. These include pelotretis flavilatus Waite, 1910, Chrysophrys auratus (Forster, 1801), 
Cheilodactylus macropterus (Forster, 1801), Congiopodus

leucopoecilus (Richardson, 1846) and Acanthoclinus quadridactylus. (Forster, 1801).

31. Ruck, J. G. 1971. Development of the lumpfish Trachelochismus melobesia (Pisces: Gobiesocidae). Zool. Publs Vict. Univ. Wellington, (57): 9 pp., 4 fig.- Deals with the embryological and prolarval development.

32. Ruck, J. G. 1973a. Development of Tripterygion capito and Tripterygion robustum (Pisces: Tripterygiidae). Zool. Publs Vict. Univ. Wellington, (63):l-10, 3 fig.- The embryological development through to the prolarval stage is described in detail for both species.

33. Ruck, J. G. 1973b. Development of the clingfishes Diplocrepis puniceus and Trachelochismus pinnulatus (Pisces: Gobiesocidae). Zool. Publs Vict. Univ. Wellington, (64):1-12, 4 fig.- The embryological development through to the prolarval stage is described in detail for both species. Later larval stages are described for D. puniceus (Richardson, 1846).

34. Thomson, G. M. 1905. The Portobello marine fish-hatchery and biological station. Trans. Proc. N.Z. Inst., 38:529-558, 5 tab., 5 pl. The history and construction of the hatchery is described, along with data and illustrations on the eggs and larvae of Armotretis rostratus Gunther, 1876, Parapercis colias (Forster, 1801) and Congiopodus. leucopoecilus (Richardson, 1846).

35. Thomson, G. M. and Anderton, T. 1921. History of the Portobello Marine Fish-hatchery and Biological station. Bull. Bd Sci. Art. N.Z, , (2): 1.31pp. - Varied embryological and larval data with figures, on several New Zealand fish. The first two are described more fully than the rest.

Parapercis colias (Forster, 1801)

Chelicionichthys kumu (Lesson and Garnot, 1826)

Lepidotrigla brachyoptera Hutton, 1872

Congiopodus Iencopoecilus (Richardson, 1846)

Helicolenus percoides (Richardson, 1845)

Peltorhamphus novae-zeelandiae Gunther, 1862

Pelotretis flavilatus Waite, 1911

Amotretis guntheri (Hutton, 1873)

Nhombosolea plebeia (Richardson, 1843)

R. tapirina Gunther, 1862 
36. Vooren, C. M. 1972. Postlarvae and juveniles of the tarakihi Teleostei: Cheilodactylidae) in New Zealand. N.Z. J1 mar. Freshwat. Res., 6(4):602-618, 9 fig., 2 tab. The postlarvae and juveniles of Cheilodactylus macropterus (Bloch and schneider, 1801) are described and figured, including information on their distribution.

37. Webb, B. F. 1973. Fish populations of the Avon-Heathcote Estuary,

2. Breeding and gonad maturity. N.Z. Jl mar. Freshwat. Res., $7(1 \& 2): 45-66,26$ tab. - Includes brief reference to the mode of deposition of fertilized eggs of some common estuarine fish.

38. Young, N. W. 1927. The spawning of the sea-horse. N.Z. J1 Sci., 8:289. - Includes observations about the brooding by the male and subsequent release of the larval. forms.

\section{Ereshwater larvae}

39. Benzie, V. L. 1961. A comparison of the life history and variation in two species of Galaxias, G. attenuatus and G. vulgaris. M.Sc. thesis, University of Canterbury, New zealand. (See 1968 publications).

40. Benzie, V. L. 1968a. Some ecological aspects of the spawning behaviour and early development of the common whitebait. Galaxias maculatus attenuatus (Jenyns). Proc. N.Z. ecol. Soc., 15:31-39, 13 fig., 1 tab.- Includes data on the effect of environmental factors, in particular temperature, on the incubation of the eggs. Developmental stages figured.

41. Benzie, V. I. 1968b. A consideration of the whitebait stage of Galaxias maculatus attenuatus (Jenyns). N.Z. J1 max. Freshwat. Res., 2(3):559-573, $4 \mathrm{fig} ., 5$ tab. - Includes a study of the size composition of a seasons catch of $\mathrm{G}$. maculatus attenuatus (Jenyns, 1842) whitebait. Information is given on the moxphometric changes that occur as the vhitebait develops (sizes, pigmentation and osteology). Galaxias maculatus attenuatus whitebait axe compared with those of G. fasciatus Gray, 1842, G. brevipinnis Glinther, 1866 , G. postvectis Clarke, 1899.

42. Benzie, V. L. 1968c. Stages in the normal development of Galaxias maculatus attenuatus (Jenyns). N.Z. J.l max. Freshwat. Res., 2(4): 606 627, 13 fig.- A very full description of the embryological. and prolarval stages. 
43. Benzie, V. L. 1958d. The life history of Galaxias vulgaris Stokell, with a comparison with G. maculatus attenuatus. N.Z. J1 mar. Freshwat. Res., 2(4): 628-653, 12 fig., 4 tab.- The embryological to prejuvenile stages of development of G. vulgaris stokell, 1949 are described and figured in detail. This development is compared with that of $\mathrm{G}$. maculatus attenuatus.

44. Dickinson, P. 1951. Field notes for the freshwater naturalist. Handb. Dom. Mus. N.Z., 3:1-37, figs.- Very brief information on spawning, hatching and migration of Galaxias attenuatus.

45. Eldon, G. A. 1969. Observations on growth and behaviour of Galaxiidae in Aquariums. Tuatara, 17(1):34-46.- General information about keeping the "whitebait" stages of several galaxiid species in aquaria.

46. Eldon, G. A. 1971. Suspected terxestrial deposition of eggs by Neochanna apoda Günther (Pisces: Salmoniformes: Galaxijdae). J. Fish. Biol., 3:247-249, 1 pl. - The eggs of N. apoda Günther, 1867 are found deposited above the watexline in an aquaxium. A terrestrial deposition of these eggs in the wild is postulated.

47. Gibson, E. 1903. Notes on New Zealand whitebait. Trans. Proc. N.Z. Inst., 35:311. - Brief information about keeping the whitebait of G. maculatus (inanga) in aquaria.

48. Harris, R. J. 1948. Further report on bully breeding. N.Z. Aquar. Bul1., 10(7):50.- A brief note about newly hatched larvae of the "Cock-a-Bully" in aquaria.

49. Hector, J. 1903. Notes on New Zealand whitebait. Irans. Proc. N.Z. Inst., 35:312-319.- Deals with the question of what whitebait are, and assigns G. attenuatus (G. maculatus) (Jenyns, 1842) as the main source. He suggests prototroctes oxyrhynchus (Günther, 1870) and Retropinna spp. as a further source of whitebait.

50. Hefford, A. E. 1928. Whitebait. Rep. Fish. N.z. 1928: 14-18.- This series through to 1934 contain data on whitebait catches throughout New Zealand.

51. Hefford, A. E. 1931a. Whitebait. Rep. Fish. N.Z. 1930: 15-16, 21-23.Additional information about $\mathrm{G}$. attenuatus (G. maculatus) and its contribution to whitebait runs. Brief mention of spawning grounds, incubation times and hatching which coincides with high spring tides. Research carried out by Captain Hayes.

52. Hefford, A. E. 1931b. Whitebait. Rep. Fish. N.Z. 1931: 14, 16-17.Need for protection of the spawning grounds from cattle grazing. 
53. Hefford, A. E. 1932. Whitebait investigation. Rep. Fish. N.Z. 1932: 11-15. - A continuation of work by Captain Hayes, relating to the growth of whitebait in aquaria. Concern is expressed about the effect of weed-killers and draining operations on spawning and incubation grounds.

54. Hefford, A. E. 1934a. Whitebait. Rep. Fish. N.Z. 1933: 13-14.

55. Hefford, A. E. 1934b. Whitebait. Rep. Fish. N.Z. 1934: 14-15.

56. Hope, D. 1927a. Whitebait (Galaxias attenuatus). Rep. Nth. Cant. Accl. Soc., 63: 2pp.- Brief comments on the whitebait as the young of the "inanga", Galaxias attenuatus, and its value as trout food.

57. Hope, D. 1927b. Whitebait (Galaxias attenuatus): growth and value as trout food. Trans. Proc. N.z. Inst., 58: 389-391, 2 pl.Figures the development of G. attenuatus from the larval stage to aduIt, and confirms $G$. attenuatus as the main contributor to whitebait masses.

58. Hopkins, C. I. 1971. Life history of Galaxias divergens (Salmonoidea: Galaxidae). N.Z. J. mar. Freshwat. Res., 5(1): 41-57, 7 fig., 7 tab.- The embryonic, prolarval, larval and prejuvenile stages are described. Embryology and prolarvae figured.

59. Mackenzie, A. G. 1903. Notes on the whitebait of New Zealand. Trans. Proc. N.Z. Inst., 35: 309-310.- Mackenzie considers the whitebait a separate species, and not the young stages of Galaxias attenuatus.

60. MCDonald, I. 1947. Further report on swarming fish in the Wanganui River. N.z. Aquar. Bull., 10(2):11.- Scant information about migration of small fish (probably whitebait).

61. MCDowall, R. M. 1963. Studies on the biology of the New Zealand fxeshwater red-finned bully Gobiomorphus huttoni (Ogilby) (Eleotridae). M.Sc. thesis Victoria University of Wellington New Zealand.- See later references $1965 b$.

62. MCDowa11, R. M. 1964a. The affinities and derivation of the New Zealand freshwater fish fauna. Tuatara, 12(2): 56-67.- Mention is made of the maxine larval phases of several New Zealand freshwater species, and their importance in relation to geographical distribution of the adults.

63. MCDowal1, R. M. 1964b. A consideration of the question "what are whitebait?" Tuatara, 12(3): 134-146, figs.- An abbreviated form of information in McDowall (1970). 
64. McDowal1, R. M. 1965a. The composition of the New Zealand whitebait catch, 1964. N.Z. J1 Sci., 8:285-300, 10 fig., 2 tab.A consideration of the full range of creatures that make up the whitebait catches.

65. McDowal1, R. M. 1965b. Studies on the biology of the red-finned bully Gobiomorphus huttoni (Ogilby) II - Breeding and life history. Trans. R. Soc. N.Z., Zool., 5(14): 177-196, 6 fig., 1 tab.- The complete developmental sequence from the fertilised egg to the juvenile is described and figured in detail.

66. McDowall, R. M. 1966. Further observations on Galaxias whitebait and theix relation to the distribution of the Galaxidae. Tuatara, $14(1): 12-18,2$ fig.- The whitebajt stage of G. argenteus (Cnelin, 1789) is described and figured.

67. McDowal1, R. M. 1968. Galaxias maculatus (Jenyns), the New Zealand whitebait. Fish. Res. Bull. N.Z., (2): 83pp., 43 fig., 8 tab., 1 p1.- The whitebait stage is described and figured, along with considerable infomation on seasonal migration. Spawning and incubation are described and the early larval stages figured.

68. MCDowall, R. M. 1969. Lunar rhythm in aquatic animals. A general review. Tuatara, 3: 133-144.- A consideration of the effect of spring (noon) tides on incubating eggs of G. maculatus.

69. McDowall, R. M. 1970. The Galaxiid fishes of New zealand. Bull. Mus. Comp. Zool. Harv, $139(7)$ : 341-432, 45 fig., 7 tab. Attention is given to the prejuvenile and juvenile stages of G. maculatus, G. fasciatus, Gray, 1842, G. brevipinnis, G. postvectis and G. argenteus. Considerable information on the life history of these and other related species is present throughout the text.

70. McKenzie, D. H. 1904. Whitebait at the Antipodes, N.Z. Illustr. Mag., $10: 122--126,4$ fig. A dramatic account of the spawning and whitebait migration of Galaxias attenuatus.

71. McKenzie, M. K. 1933. Embryonic and larval structures of Galaxias attenuatus (Jenyns). Published M.Sc. thesis, Victoria University of Wellington, New Zealand.- Information herein is published in Benzie $1968 \mathrm{C}$.

72. McMillan, H. M. 1961. A contribution to the knowledge of the fish Retropinna anisoden Stokell. Trans. R. Soc. N.Z., Zool., 1(10): 139-144, 2 fig.- The embryological development is figured and described. A 4.8 mon prolarva is also figured but not described. 
73. Morrison, I. A. 1952. Whitebait, thejr life and habits. N.Z. Fish. Sh. Gaz., 19(4):21, 1 fig.- Contains general information about whitebait runs of the 'inanga', Galaxias attenuatus.

74. Phillipps, W. J. 1919. Life history of the fish Galaxias attenuatus Aust. Zool., 1(7):211-213.- General information about spawning, incubation and migration.

75. Phillipps, W. J. 1924. The New Zealand minnow. N.Z. JI Sci. Technol., 7:117-9.- A brief mention of the larval and prejuvenile runs of G. attenuatus, Retropinna retropinna (Richardson, 1845) and Gobiomorphus gobioides (Cuvier and Valenciennes, 1837). Also includes information on the habits of $\mathrm{G}$. attenuatus larvae and prejuveniles in the aquarium.

76. Powell, L.1870. On four fishes commonly found in the River Avon wi.th a consideration of the question, what is whitebait? Trans. Proc. N.Z. Inst., 2:84-86, 2 fig.- The pigmentation of the prejuvenile whitebait is described.

77. Skrzynski, W. 1968. The Canterbury mudfish, Galaxias burxowsius Phillipps, a vanishing species. N.Z. J1 mar. Freshwat. Res., $2(4): 688-697,3 \mathrm{fig} ., 1 \mathrm{tab}$. - Includes a brief description of a prejuvenile $G$. burrowsius.

78. Whitley, G. P. 1935. Whitebait. Victorian Nat. Melb., 52(3):41-51, 1 pl.- General information on spawning, incubation and migration of Galaxias attenuatus.

79. Whitley, G. P. 1956. The story of Galaxias. Aust. Mus. Mag., 12(1): 30-34.- Very brief information about the catching of "whitebait" of "Austrocobitus (Galaxias) attenuatus".

80. Woods, C. S. 1966. Species composition of whitebait (Galaxias spp.). Rec. Canterbury Mus., 8:177-179.- This contains a list of the adults which contribute to the whitebait shoals.

81. Woods, C. S. 1968. Growth characteristics, pigmentation, and the identification of whitebait (Galaxias spp., Salmonoidea). N.Z. J1 mar. Freshwat. Res., 2(2): 162-182, 13 fig.- Attention is given to the prejuvenile and juvenile stages of $G$. maculatus attenuatus, G. brevipinnis, G. fasciatus, G. postvectis and G. argenteus, with emphasis on the development of pigmentation and its use in identification. 
Appendix II

A light-trap for collecting marine fish laxvae and invertebrates

By J. G. Ruck

Zoology Department, Victoria Univexsity of Wellington

\section{Introduction}

Lights have often been used as aids in collecting various marine and freshwater organisms. These exploit the positive phototropism exhibited by certain spccies e.g. cumaceans (Hale, 1953), aquatic insect larvae (Hungerford et al., 1955; Washino and Hokama, 1968), assorted freshwater invertebrates (Espinosa and Clark, 1972), marine invertebrates (Sheard, 1941) and fish larvae and juveniles (Winn and Miller, 1954; Parsons and Hodder, 1970). Various systems have been used, from suspended lights and dipnets (Winn and Miller, 1954) to moxe elaborate submersible and semi-submersible traps (Hungerford et al., 1955; Washino and Hokama, 1968; Espinosa and Claxk, 1972).

The light-trap described here was designed for collecting larval. and prejuvenile fish of the families Tripterygiidae ("blennies"), clinidae and Gobiesocidae ("clingfish"). The trap was to be used close inshore from rocks, wharves and boats. Hence it needed to be light yet robust enough to withstand the normal wearmand-tear of field handling. The light-traps referred to earlier that were designed for collecting aquatic insects etc, were too small. to collect large numbers of fish larvae. A trap similax to that used by Davies (1954) and Baker (1972) for collecting pilchard larvae was available. However, this was too cumbersome for my purpose. Furthermore, the total collecting area of the cones was small relative to the area of the trap radiating light, thus decreasing the catching efficiency. I have used a sealed light on the end of a long pole, the light being placed just under. the water to attract fish larvae that were then captured by a dipnet. This method, while effective in rocky shore surge channels not suitable for set traps, did not give any quantitative assessment, however rough, of larval number. Furthermore, light traps have an advantage in that they can be left unattended for several hours. 
The trap needed to be submersible to different depths, portable and usable in the sea. Certain basic problems therefore had to be overcome. These were principally the sealing of the light, corrosion, release of water when retrieving the trap, loss of or damage to specimens, and weight of water causing stress on the apparatus. In addition to solving these problems, it was necessary to devise a system whereby the greatest cone aperture area (effective collecting area) was presented to the organisms. Perspex, because of its transparency and durability, was chosen as the main building material.

\section{Description and Method of Construction}

The trap (Fig. $4 \& 5$ ) is essentially a squat pexspex "box" with sides that form the outer aperture of four cones. The cones taper to narrow slit-like inner apertures that are in line with a light in the center of the apparatus. All sides of the trap, therefore, act as effective collecting areas. The top of the "box" is opaque to restrict radiating light to the areas of the cones; organisms are then not likely to approach the light from above the trap but rather from the side in line with the cones. The gauze "cod-end" (bottom of the "box") is four-sided and tapered, ending in a terylene collar designed to hold an Agee jar (Fig. 5). The corners are joined and strengthened by terylene tape. The upper edge of the net is bordered by a $3 \mathrm{~cm}$ wide terylene strip, folded from a $6 \mathrm{~cm}$ strip. The size of the mesh used for the "cod-end" will depend on the size of the animals to be collected (300 microns for this trap), but mesh size must be large enough to allow reasonably rapid drainage of water from the light-trap. If not the weight of the water in the "cod-end" will place undue stress on the whole apparatus. A mesh "cod-end", as distinct from some solid equivalent, has the added advantage of being easily folded. The trap is thereby more readily handled.

The sealed light unit is fastened to the centre of the laminated top ( $\mathrm{Fig} .4 \& 5, \mathrm{~A}_{1}$ and $\mathrm{A}_{2}$ ) and gives maximum radiation of light through the cones. The light (Fig.1) consists of a bxass holder, the lower portion of which is hollow and threaded to accommodate a small jam jar. This is sealed by pressure against a flat rubber ring. The flex enters the jar through the upper cylindrical stem of the brass holder, and effective sealing 
is obtained with an "O-ring" forced tightly about the flex by a short bolt. Vaseline is spread liberally over the seals and the rim of the jar. Inside the jar there is a simple brass bulb holder that is screwed firmly to the brass unit, and recejves the two wires from the flex. Klinger (in Hale, 19532 used a 5000 watt light hoping to attract a great variety of marine life. Instead he found this very strong light less effective in attracting organisms than a simple hand held flash light. Foxon (1936) and Hale (1953) both found that cumaceans were attracted most effectively by low intensity light. They found that cumaceans tended to shun the brightest areas, preferring to remain on the periphery of the radiated light. A low intensity light (12v6w) was therefore chosen for this trap.

The cylindrical projection of the brass light holder passes through the laminated top of the trap and is held securely in place by four screws. The light-trap is suspended by four bridles each $54 \mathrm{~cm}$ long with spliced loops at each end. How the bridles are attached to the trap is a matter of personal choice; in this case aluninium U-sections were used (Fig.3, no.1). The free ends of the bridles are gathered together and fastened to a galvanised shackle, which in turn is attached to the $10 \mathrm{~m}$ waxp. The length of the warp will. vary according to the height of the wharf or boat and the depth of the water to be sampled. The flex from the light-unit is attached at intervals to the warp. The light trap is constructed in the following way:

(1) Cut the collecting cones, top and sides (Fig. $2 \& 5 \mathrm{~A}-\mathrm{E}$ ) from a single sheet of $5 \mathrm{~mm}$ thick clear perspex measuring at least $116 \mathrm{x} 91 \mathrm{~cm}$ (Fig. 2). When cutting allow for the width of the saw cuts. Smooth or bevel the rough edges using medium (not fine) sandpaper.

(2) Form the top of the trap by laminating $A_{1}$ and $A_{2}$ (Fig. 5). As an alternative the top may be cut from a single sheet of $1 \mathrm{~cm}$ thick black perspex. If transparent perspex is used, then paint the top black. (3) Glue the sides of the cones $\mathrm{C}_{1-4}$ at right angles to the top and bisecting each corner. The edges to be glued should be bevelled to provide a larger glueing surface (Fig.3, no.2). Triangular wedges of perspex are added to increase the strength of the joints.

(4) The upper parts of the cones $B_{1-4}$ are now glued to the laminated top and to the cone sides $\mathrm{C}_{1-4}$.

(5) Glue the lower parts of the cones $B_{5-8}$ to the angled edges of the cone sides $\mathrm{C}_{1-4}$. The size of the internal cone aperture may be altered, by increasing or decreasing the angle of the upper and lower cone portions 
$\left(\mathrm{B}_{1-4}\right.$ and $\left.\mathrm{B}_{5-8}\right)$.

(6) Assemble the perspex skirt by glueing the series $\mathrm{D}_{1-4}$ to each other to form a square. $D_{3}$ and $D_{4}$ being $1 \mathrm{~cm}$ shorter lie inside $\mathrm{D}_{1}$ and $\mathrm{D}_{2}$.

(7) The skirt is now glued to the bottom edges of $B_{5-8}$ (lower edge of the cones) being further held in place by triangular perspex fillets (F) cut to fit on the inside between the skirt and the ventral plates of the cones. (8) Drill $165 \mathrm{~mm}$ diameter holes through the top $5.5 \mathrm{~cm}$ apart and lying in from a line representing the innermost border of the cones. These produce a downcurrent of water that helps prevent organisms from passing out of the cones as the trap is lifted through the water column. Further holes of similar diameter are drilled in each corner of the top, to allow air to escape from the trap as it is lowered into the water.

(9) Drill $205 \mathrm{~mm}$ diameter holes in the perspex skirt $\mathrm{D}_{1-4}$ and the backing plates $\mathrm{E}_{1-4}$ which correspond to a similar series of holes in the upper border of the gauze "cod-end". The "cod-end" may be constructed according to personal preferences and therefore requires no further explanation here. (10) Slide the terylene border of the "cod-end" over the skirt and hold in place with the backing plates $E_{1-4}$ and the $5 \mathrm{~mm} \times 2 \mathrm{~cm}$ galvanised bolts. (11) Fasten the sealed light unit to the laminated top of the light-trap. (12) Bolt the bridle attachments to the cornexs of the top of the trap, at the same time fastening the bridle, warps and flex.

\section{Materials}

Clear perspex

Perspex glue

Nylon bolting cloth

Terylene tape

Terylene cloth - collar

- border

Galvanized nuts and bolts

Self-tapping galvanized screws

Propylene rope - bridles

$$
\text { - warp }
$$

Aluminium U-section - brackets
$5 \mathrm{~mm} \times 91 \times 116 \mathrm{~cm}$ (N2 $3 \times 4$ ' stock)

$40 \times 72 \mathrm{~cm}$ (300 u mesh)

$140 \times 3 \mathrm{~cm}$

$35 \times 11 \mathrm{~cm}$

$184 \times 6 \mathrm{~cm}$

$5 \mathrm{~mm} \times 2 \mathrm{~cm}(\mathrm{x} \mathrm{20})$

$5 \mathrm{~mm} \times 3 \mathrm{~cm}(\mathrm{x} 8)$

$300 \mathrm{~cm}$

$10 \mathrm{~m}$

$1.5 \times 2 \times 16 \mathrm{~cm}$ 
1 galvanized shackle

1 sealed light unit with $12 \mathrm{v} 6 \mathrm{w}$ bulb

$112 \mathrm{v}$ battery

Insulated flex

$10 \mathrm{~m}$.

\section{Operation}

This light-trap is light and will swing about with the slightest swell. Weights, pexhaps those used by divers, may be added to each corner to increase stability. The time the trap is left submerged depends on the locality and type of animal sought. When collecting fish larvae it is advisable to restrict submersion time to no more than half an hour. Any longer and the small delicate larvae are rapidiy consumed by isopods etc., and also damaged by general overcrowding. On retrieval the trap should be brought slowly to the suxface so that the perspex skirt is just clear of the water. As the "cod-end" is lifted from the water the whole unit is moved from side to side so as to keep the organisms swimning and thus prevent them becoming entangled in the mesh sides. This factor is more important with "many-legged" crustaceans that become readily enmeshed. With the bottle clear of the water the unit may then be brought rapidly up to the wharf.

The light-trap has also been successfully used to collect amphipods (Dr A. A. Fincham, V.U.W.) and epitokous polychaetes (Mr G. Read, Marine Laboratory, Island Bay, V.U.W.). In addition various adult teleosts, zoea and megalopa larvae and isopods have been found in differing numbers in samples taken. It is likely that the unit will collect any small positively phototropic aquatic organisms.

\section{Acknowledgements}

I would like to thank Dx P.H.J. Castle, Department of Zoology, Victoria University of Wellington, for his constructive criticism of this paper. I thank Mr M. Loper, V.U.W. for the design and construction of the sealed Iight unit and Mr G. Grainger, Zoology Technician, Marine Laboratory, Victoria University of Wellington for the construction of the light-trap. 


\section{References}

Baker, A. N., 1972 .

Reproduction, early life history, and age-growth relationships

of the New Zealand pilchard, Sardinops neopilchardus (Steindachner). Fish. Res. Bull. N.Z., (5): 64 pp., 39 fig., 9 tab.

Davies, D. H. , 1954.

The South African pilchard (Sardinops ocellata). Development, occurrence and distribution of eggs and larvae, 1950-51.

Invest1. Rep. Div. Fish. Un. S. Afr., 15: 28 pp.

Espinosa, L. R. \& Clark, W. E., 1972.

A polypropylene light trap for aquatic invertebrates.

Calif. Fish Game, 58 (2): 149-152, 2 fig.

Foxon, G.E.H., 1936.

Notes on the natuxal history of certain sand-dwelling cumacea.

Ann. Mag. nat. Hist., 10 (17): 377-393, 7 fj.g.

Hale, H. M. 1943.

Notes on two sand-dwelling Cumacea (Gephyrocuma and Picrocuma)

Rec. S. Aust. Mus. 7 (4):337-342, 9 fig. 1.953.

Notes on distribution and night collecting with artificial 1 ight.

Trans. R. Soc. S. Aust., 76: 70-76, 3 fig.

Hungerford, H. B., Spangler, P. J. \& Walker, N. A. 1955.

Subaquatic light traps for insects and other animal organisms.

Trans. Kans. Acad. Sci., 58 (3) : 387-407, 2 fig.

Parsons, I. S. \& Hodder, V. M. , 1970. Occurcence of juvenile and spawning Atlantic Mackerel in Southeastexn Newfoundland coastal watexs.

J. Fish. Res. Bd Can, , 27 (11): 2097-2100.

Sheard, K. 1941 .

Improved methods of collecting marine organisms. Rec. S. Aust. Mus.,

$7: 11-14,1$ fig.

Washino, R. K. \& Hokana, Y., 1968.

Quantitative sampling of aquatic insects in a shallow-water habitat.

Ann. Ent. Soc. Amer., 61. (3): 785-786, 1 tab.

Winn, H. E, \& Miller, R. R., 1954.

Native postlarval fishes of the lower Colorado River Basin, with

a key to their identification. Calif. Fish Game, 40 (3): 273-285,

$1 \mathrm{fig}, \mathrm{l}$ tab., 4 pl. 
328.

J. G. Ruck, B.Sc. (Hons).,

Department of Zoology,

Victoria University of Wellington,

P. O. Box 196,

Wellington, New Zealand. 
Fig.1. Sagittal section of the light holder; Fig.2. Cutting plan for the main perspex sheet; Fig.3. No.1: corner bracket for the bridle; 2; bevelled edge of a right angled joint. 


Figs. 4 \& 5. Dorsal and lateral view of the completed light-trap. 


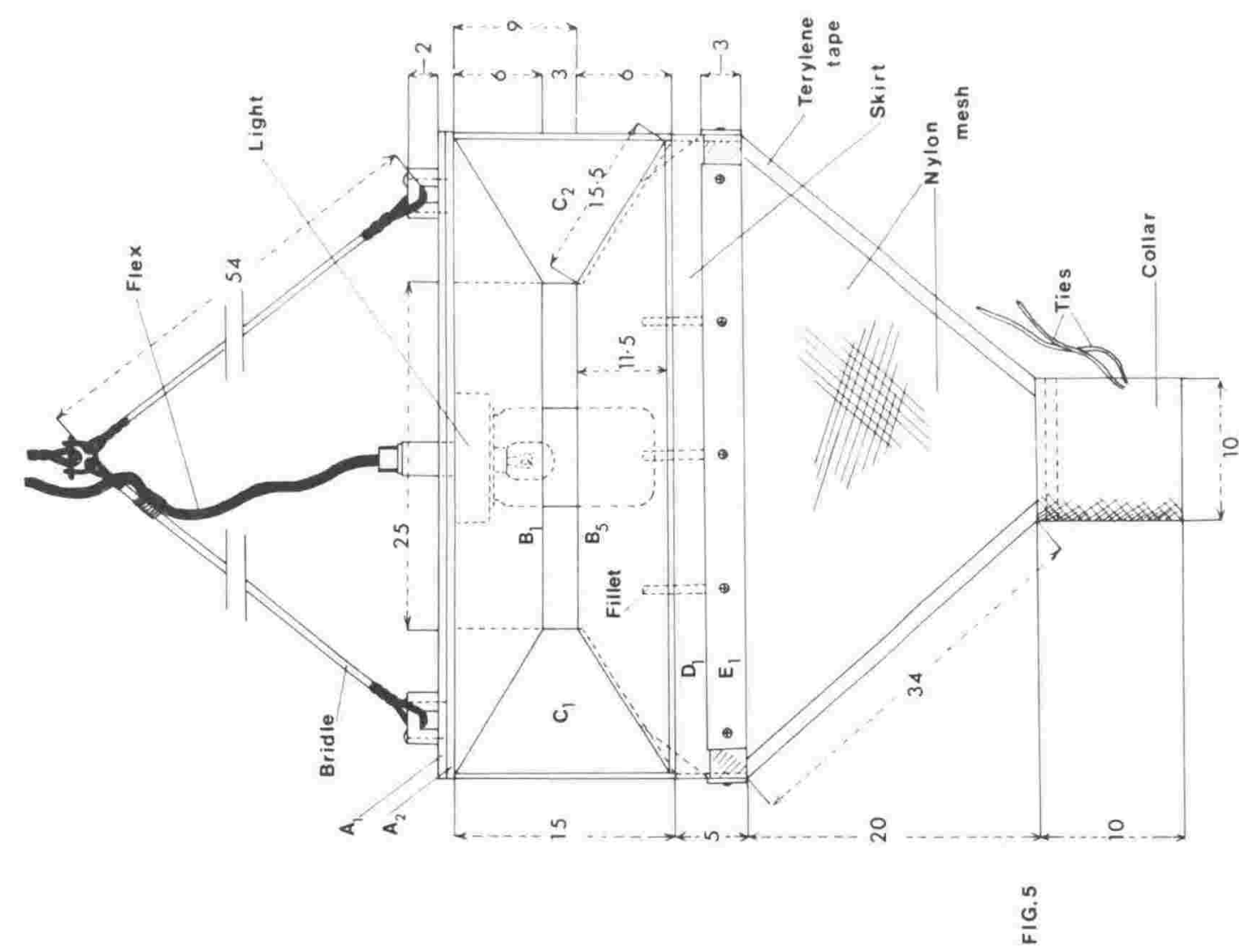

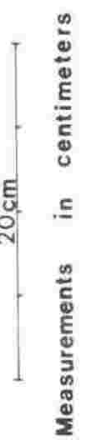

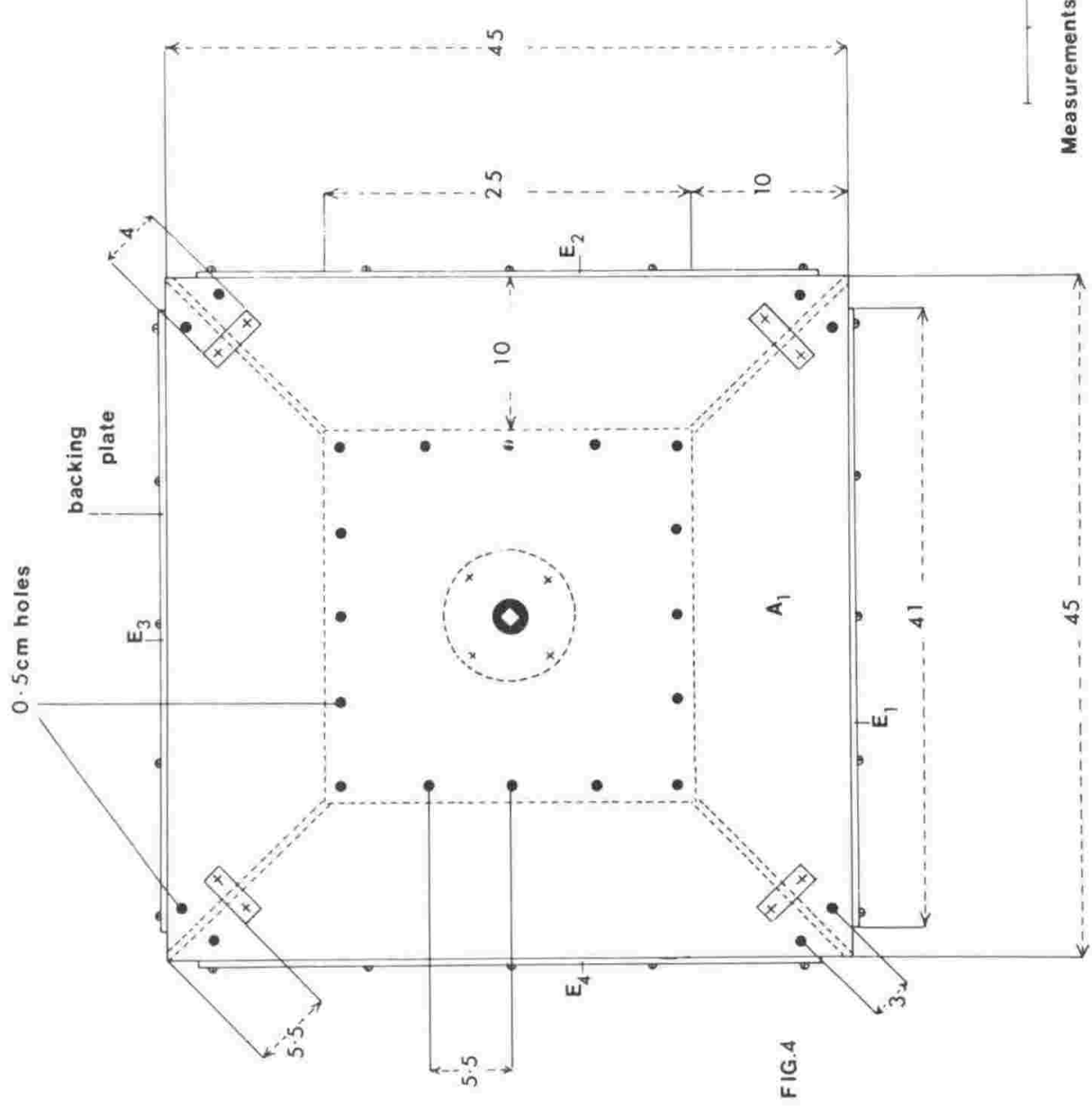




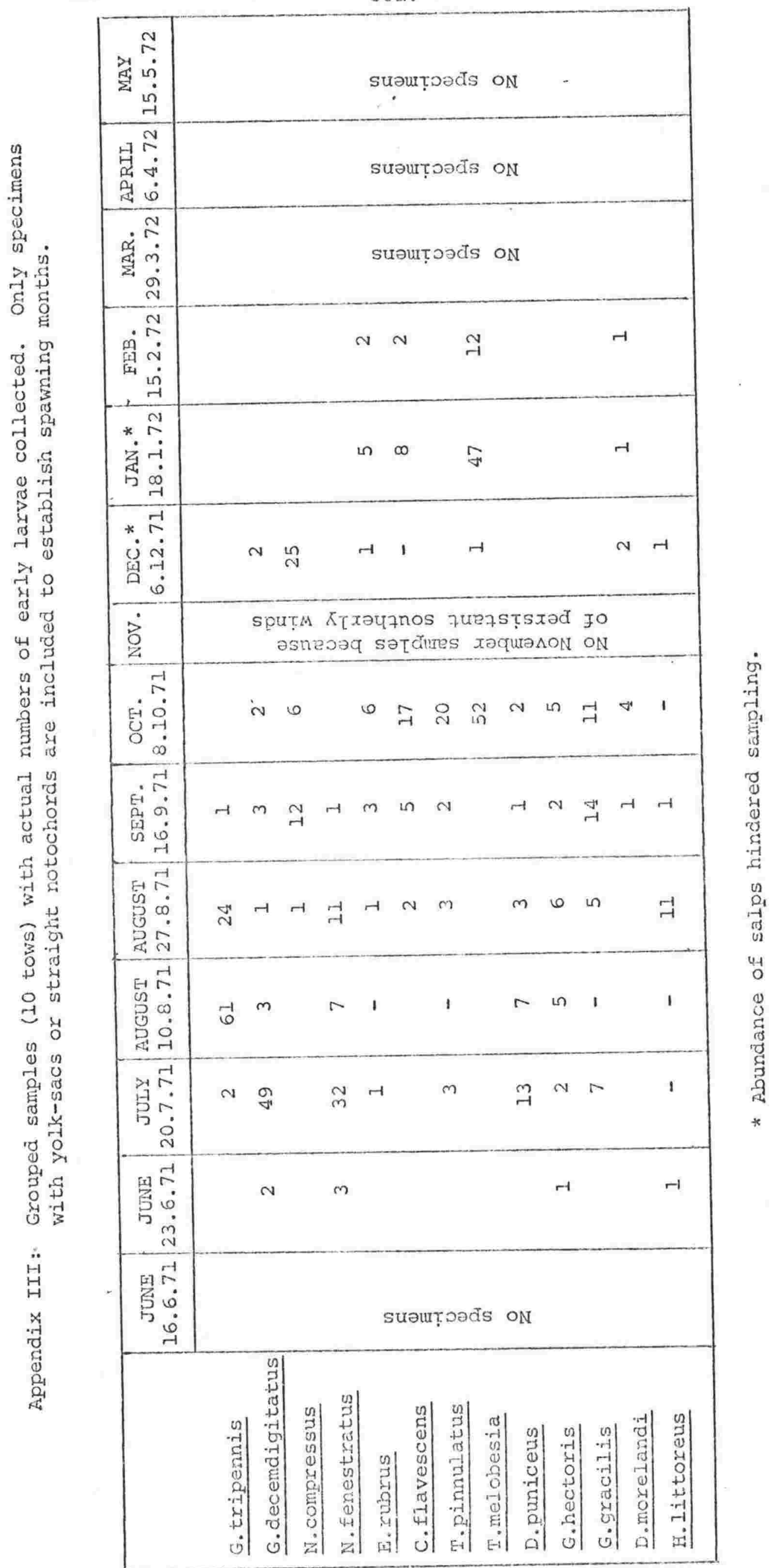




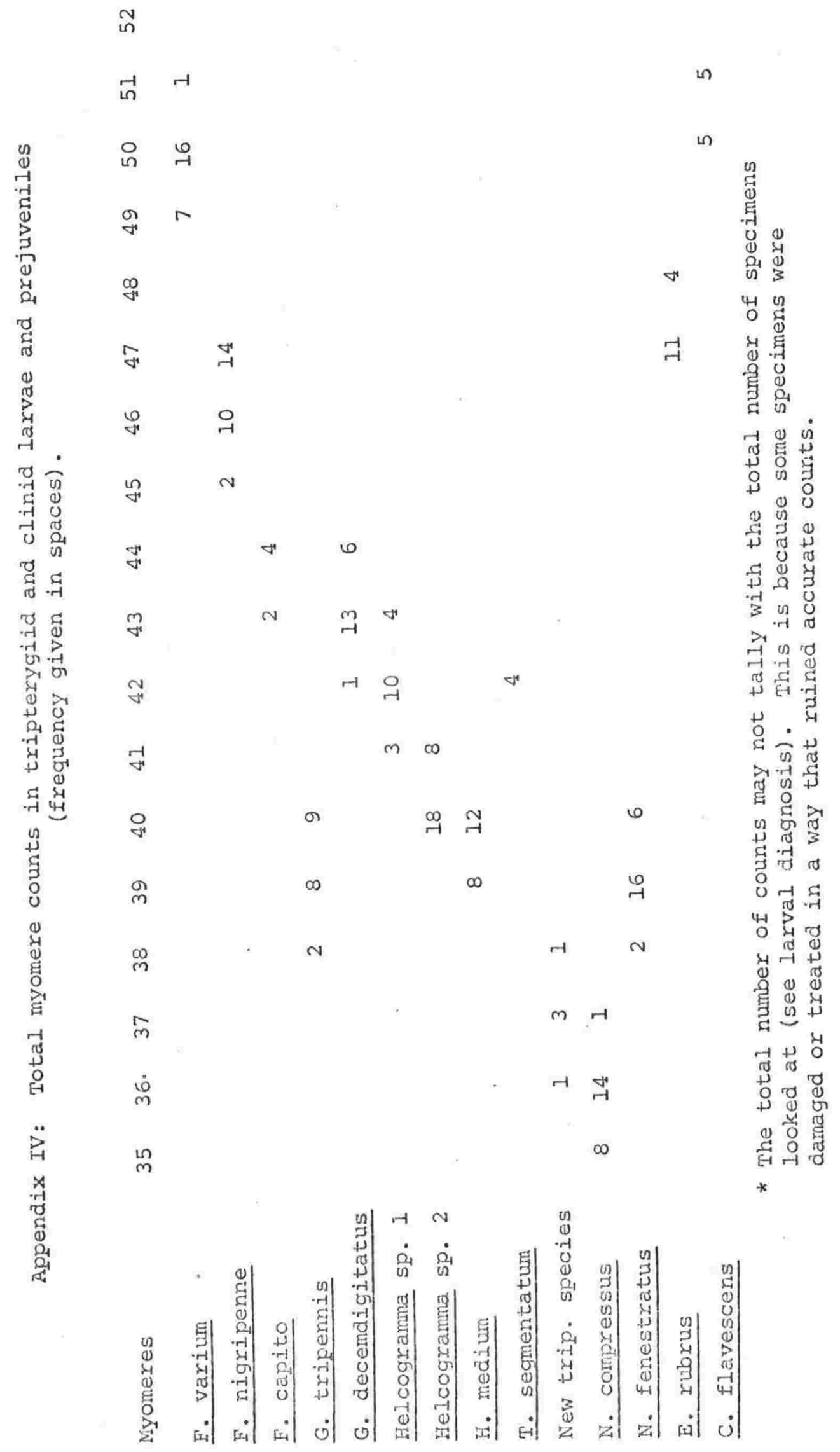




\section{오}

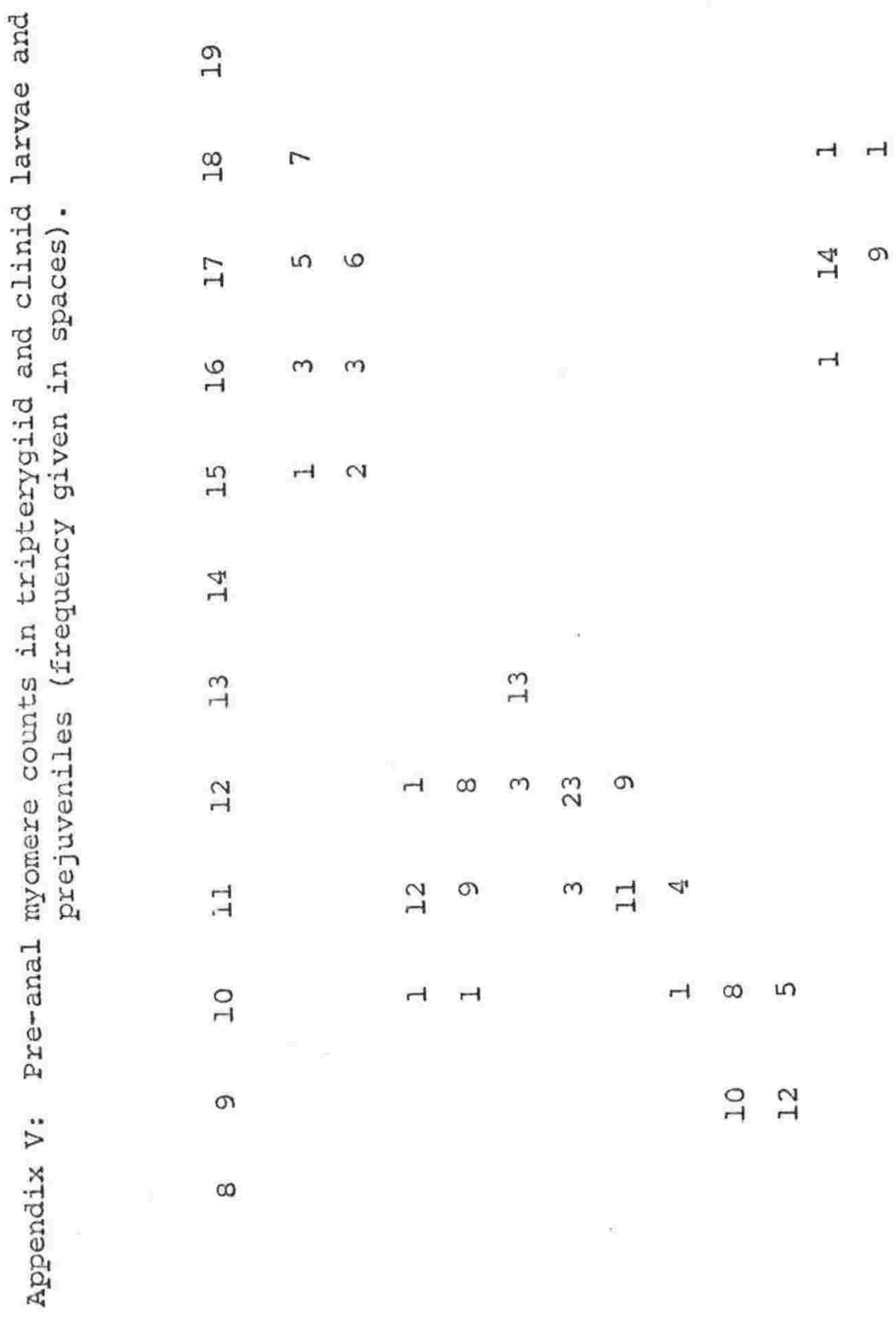




羿

가

N

ช

$\underset{? \rightarrow-1}{m}$

ข้

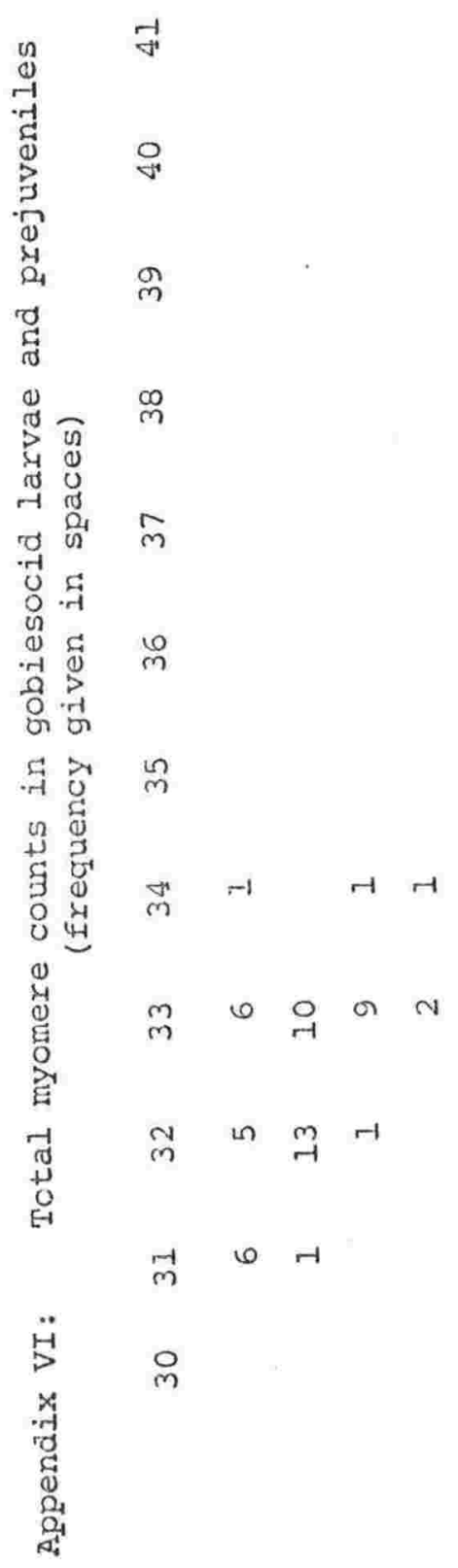

$\stackrel{\infty}{N}$

N m

它

$\stackrel{\circ}{\sim}$

$n \infty$ in

$\infty$

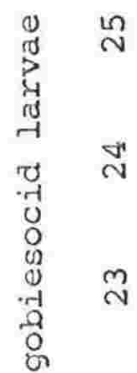

$\sim m$

㟧 N

N

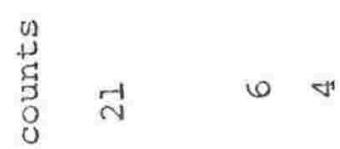

है

$\circ \infty$

공

우 군

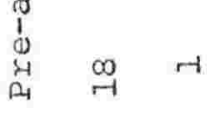

获

N

离|

离

娄

당

告

(7)

爱

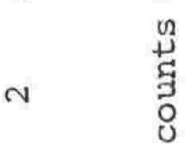

운
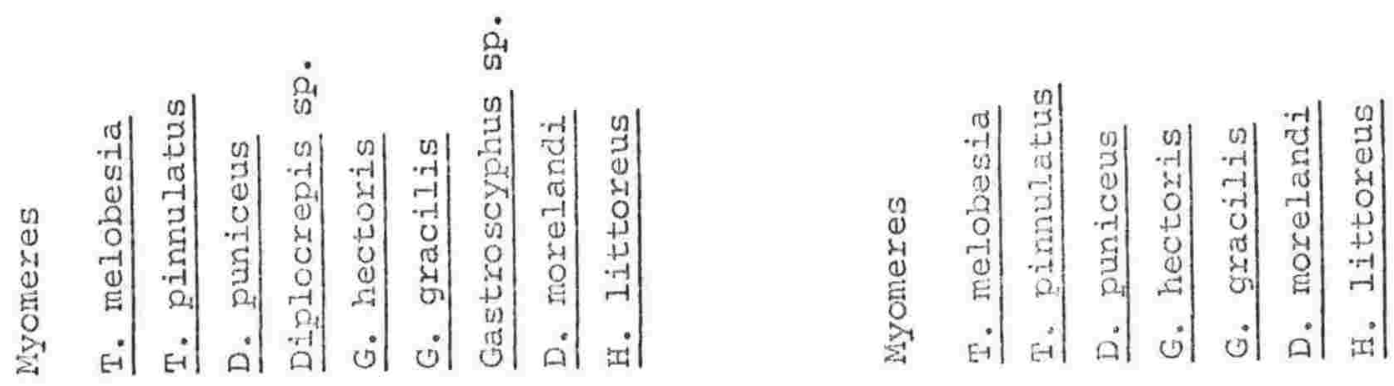


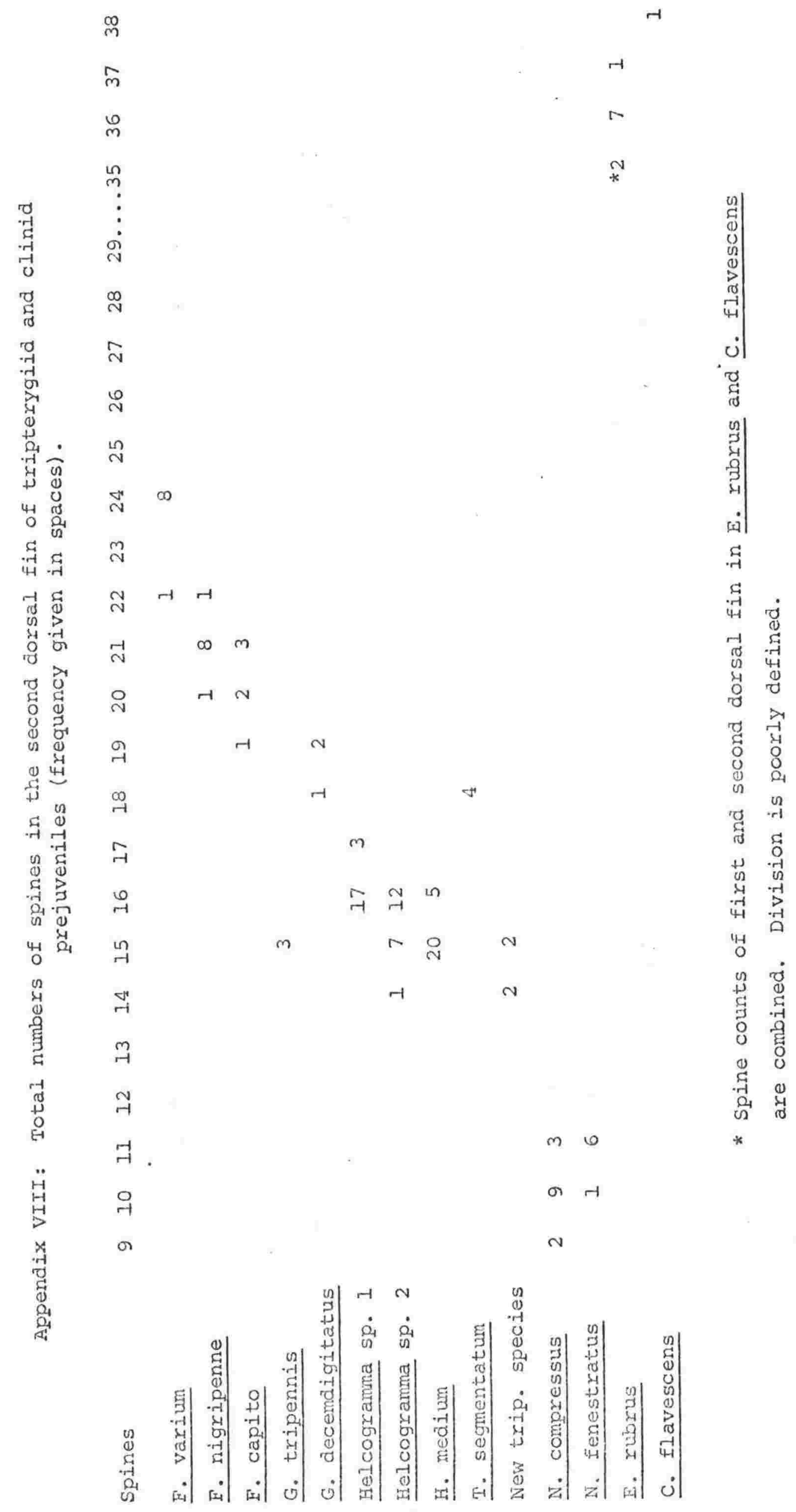


인

ำ 0

$N$

$\stackrel{\infty}{N}{ }^{-1} \rightarrow-1$

iv

ก $r$

$\stackrel{\infty}{N} \quad \infty \quad m \quad N$

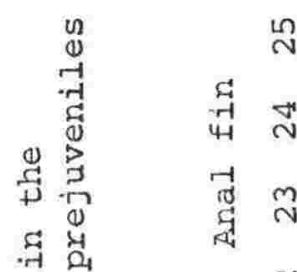

N $N$

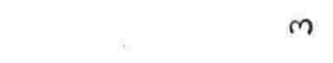

N $-1+1$

齐

वृ

त्व

.

की

of

in त्र

प्ष

० म 9

पू 制

म

出 5 है

进岳焉

त त्व

Q2 究

द

藏

出乘

on

प्र

空

究 임

rt 7

苟.



각

$\stackrel{+0}{\circ}$

$\stackrel{m}{N}$

N

ה

ㄱ

항

m

n

융

我

तु

क्ष

ज्ञ



m
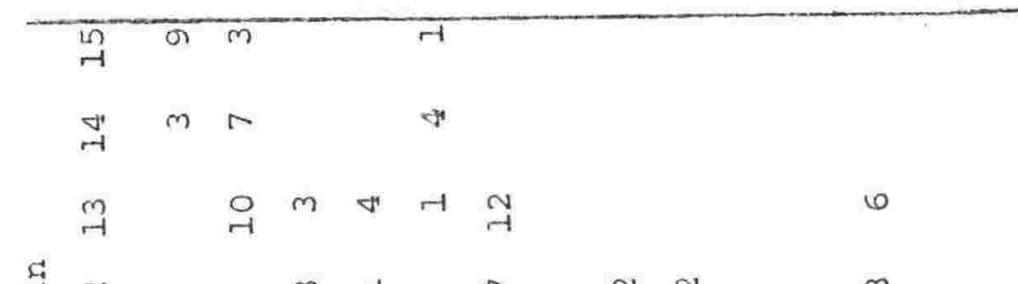

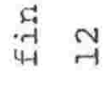

贾

पै

7
m
m
$\vdots$
$\vdots$
$\vdots$
$\vdots$

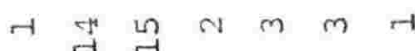

-

$\begin{array}{lll}r-1 & \text { N } & 0\end{array}$

$-1$

เก

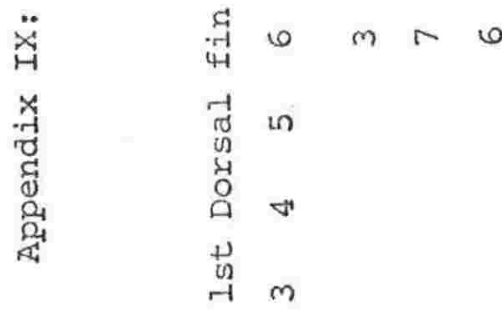

$\ln _{\rightarrow}$ ก

$m \sim$

n

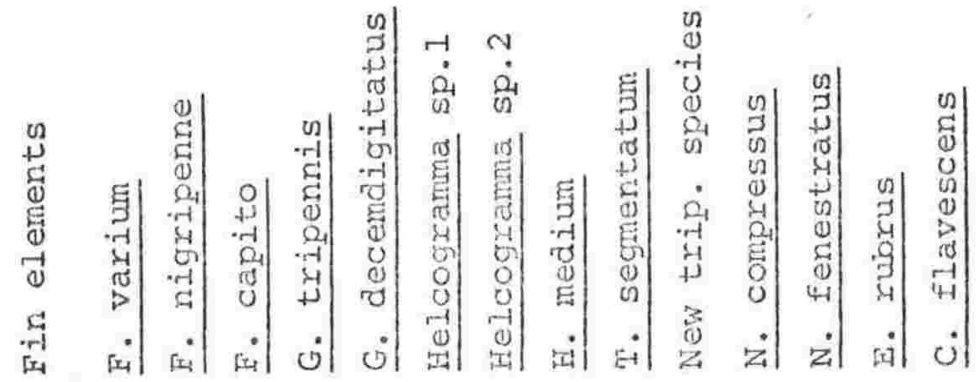




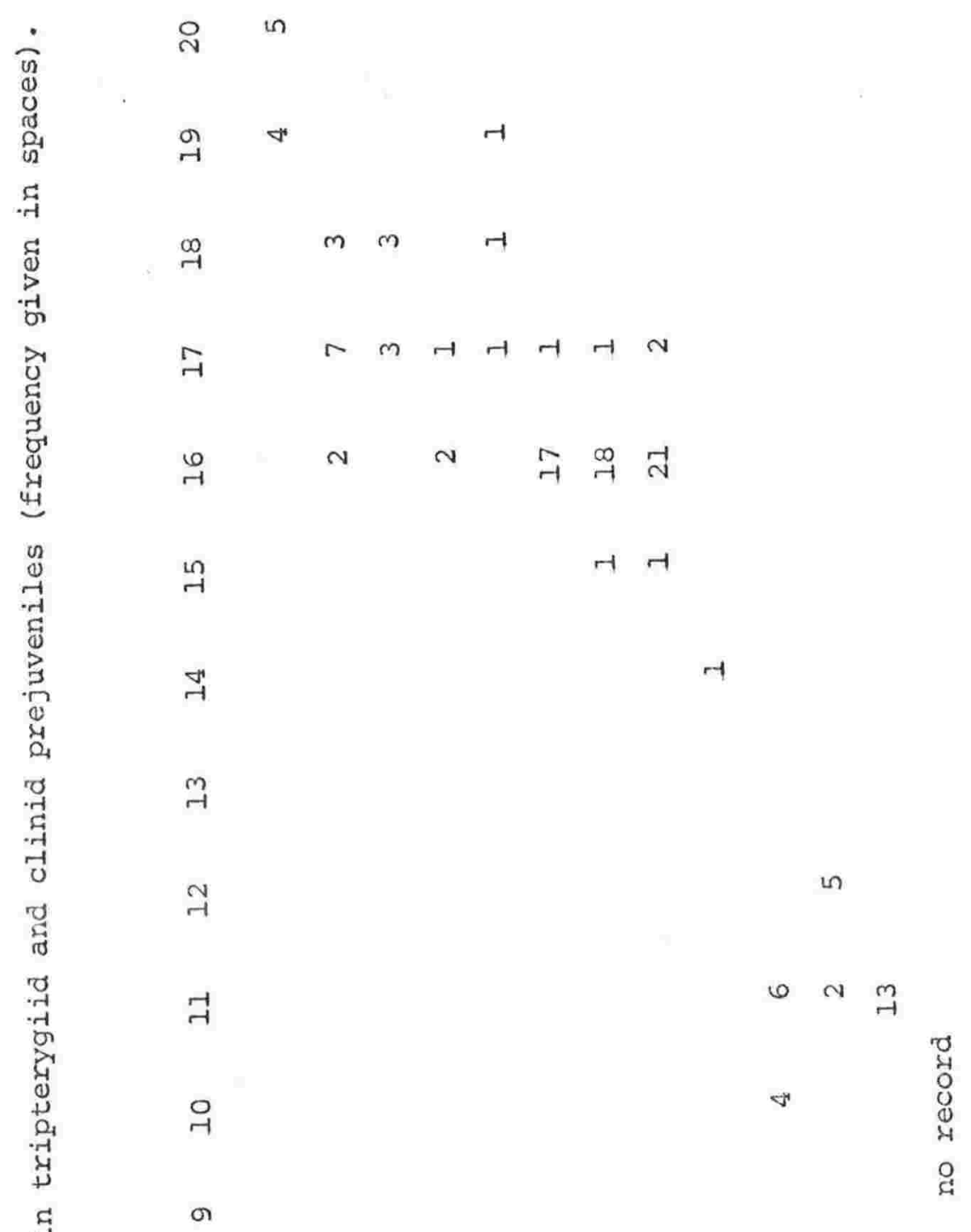

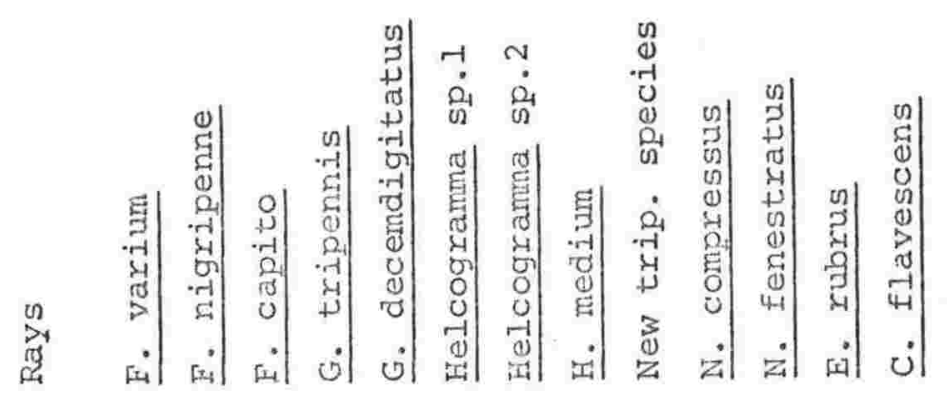




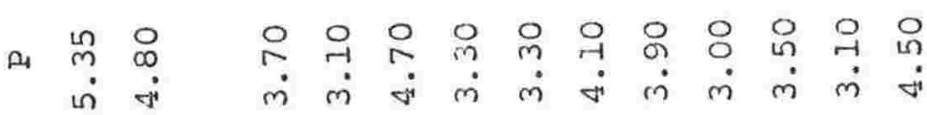

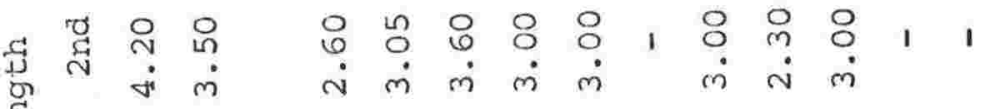

焉



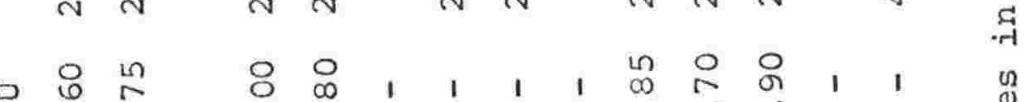

$\stackrel{5}{0}$

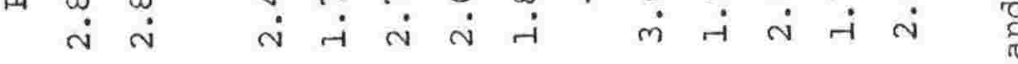

प्र

迎

๑) 品

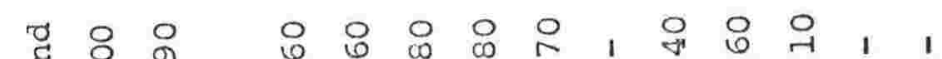

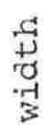

岳

प्र

त्ञ

तु

空劳

o

तี

त्

गु

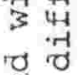

ס

is in

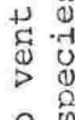

은

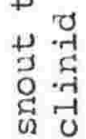

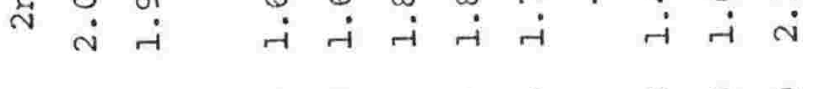

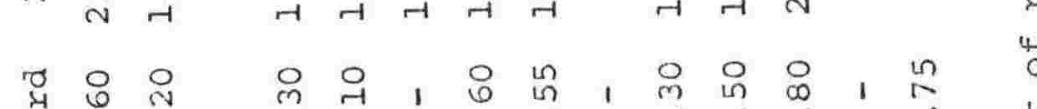

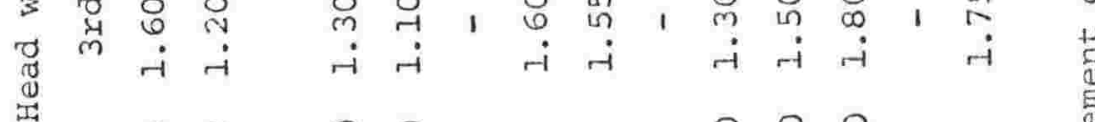

๖

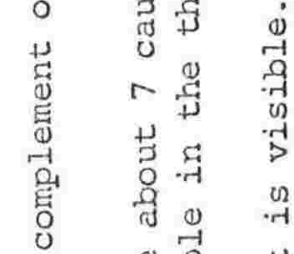

on

is

겅

究

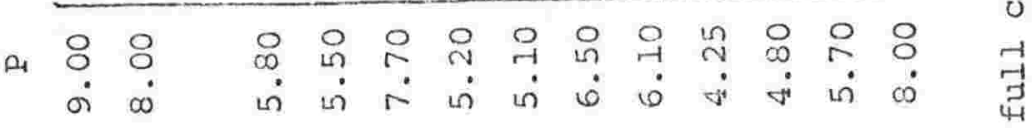

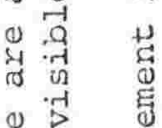

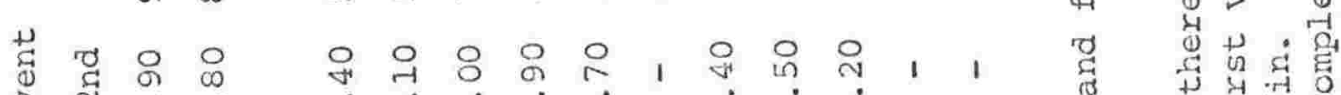

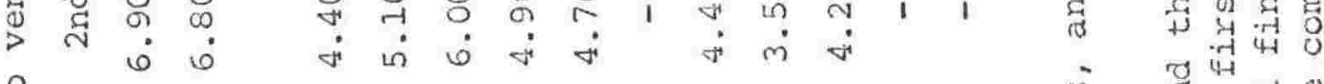
is



$$
\text { ค }
$$

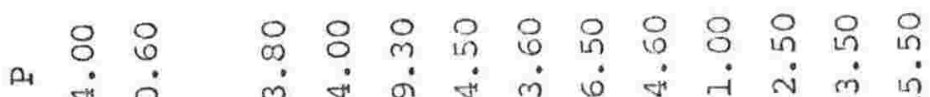

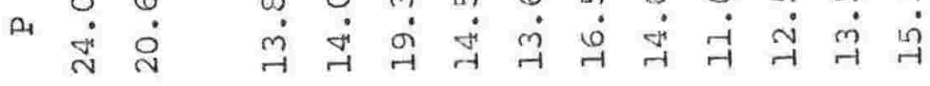

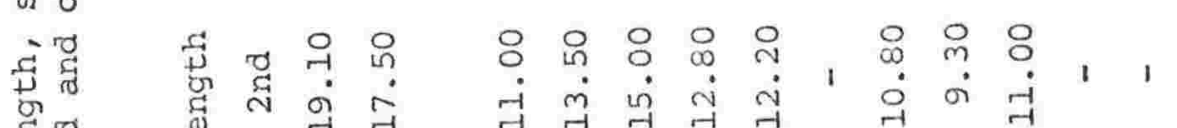
密。

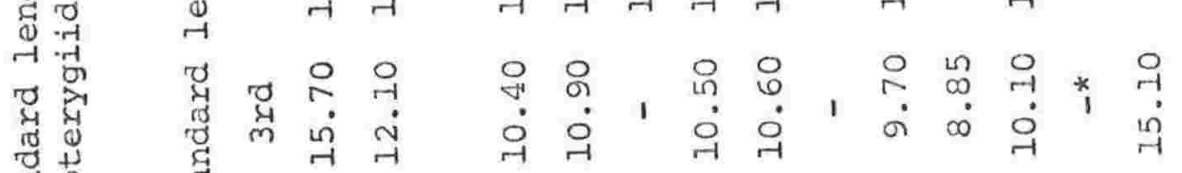
要造

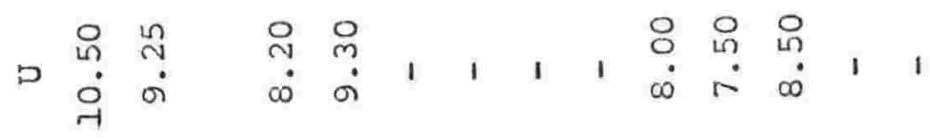

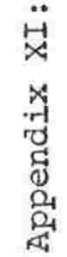

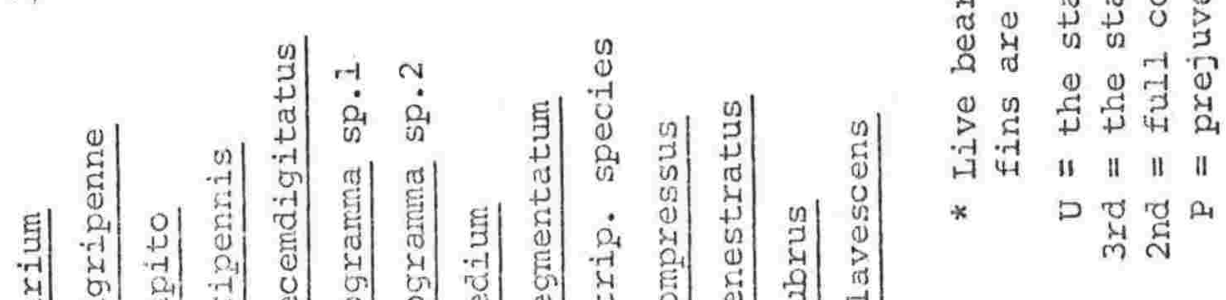

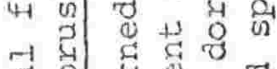
《) वे ज्ञी

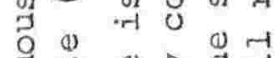
至 $\begin{aligned} & N \\ & -1\end{aligned}$

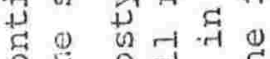

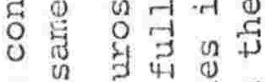
$\stackrel{0}{>} 000.7$

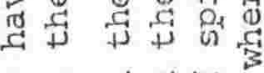

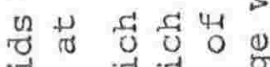

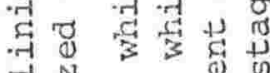
每 $N$ N क) तु त् 4 का 영

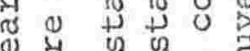
0 व 0 व

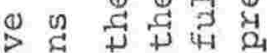
H 4 || || || || मे द्व 
号

ख्यु



वि

वृ

वै थ

运峦

or 0

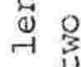

वृ

की

实

$\begin{array}{cc}-7 & \rho_{1} \\ 3 & 0\end{array}$

ro

बै

i)

स्ष

.

+ $\frac{G}{4}$

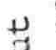

范

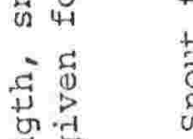

뎡 b

त-

o

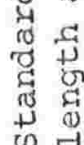

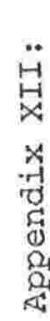

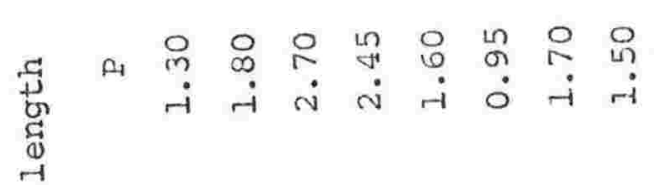

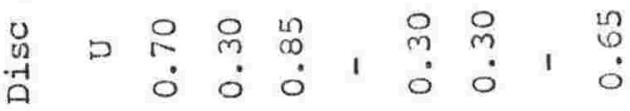

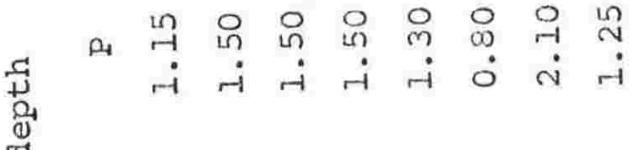

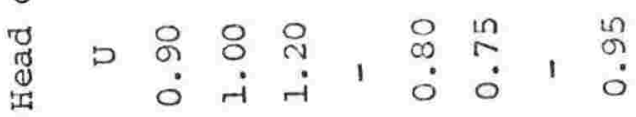

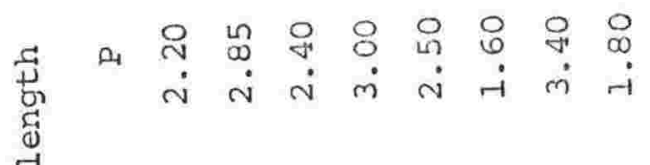

跑

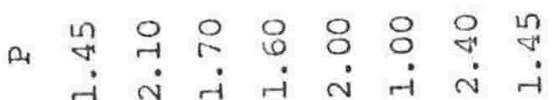

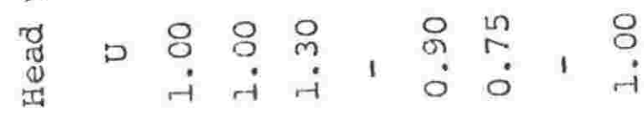

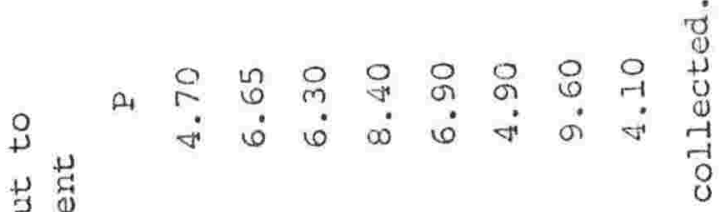

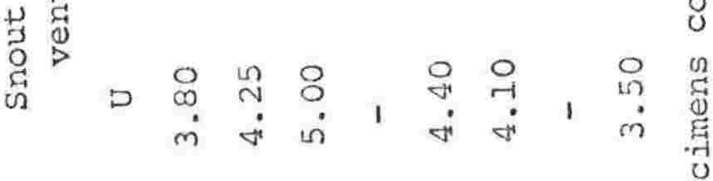

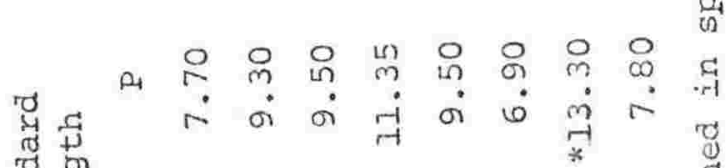

的



号

$\dot{0}_{1} \quad$ on

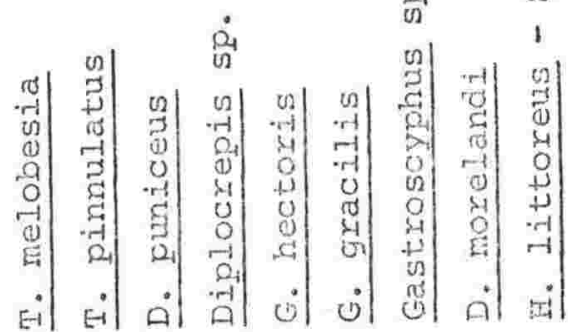

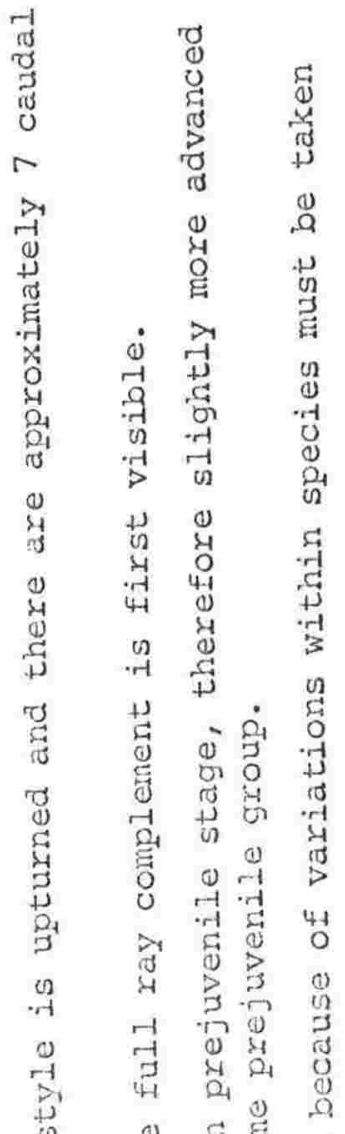

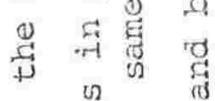

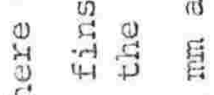



दु

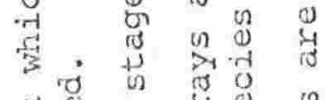

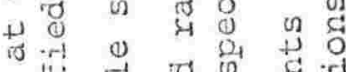
0. 出 का दा द्य

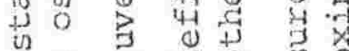

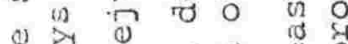

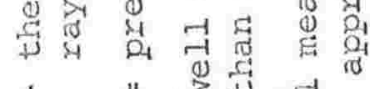
$11113 \%$ 年告 